NASA/CR-2000-210062
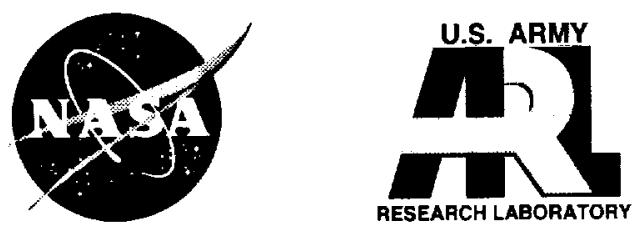

\title{
Simulating Fatigue Crack Growth in Spiral Bevel Gears
}

Lisa E. Spievak, Paul A. Wawrzynek, and Anthony R. Ingraffea Cornell University, Ithaca, New York 
Since its founding, NASA has been dedicated to the advancement of aeronautics and space science. The NASA Scientific and Technical Information (STI) Program Office plays a key part in helping NASA maintain this important role.

The NASA STI Program Office is operated by Langley Research Center, the Lead Center for NASA's scientific and technical information. The NASA STI Program Office provides access to the NASA STI Database, the largest collection of aeronautical and space science STI in the world. The Program Office is also NASA's institutional mechanism for disseminating the results of its research and development activities. These results are published by NASA in the NASA STI Report Series, which includes the following report types:

- TECHNICAL PUBLICATION. Reports of completed research or a major significant phase of research that present the results of NASA programs and include extensive data or theoretical analysis. Includes compilations of significant scientific and technical data and information deemed to be of continuing reference value. NASA's counterpart of peerreviewed formal professional papers but has less stringent limitations on manuscript length and extent of graphic presentations.

- TECHNICAL MEMORANDUM. Scientific and technical findings that are preliminary or of specialized interest, e.g., quick release reports, working papers, and bibliographies that contain minimal annotation. Does not contain extensive analysis.

- CONTRACTOR REPORT. Scientific and technical findings by NASA-sponsored contractors and grantees.
- CONFERENCE PUBLICATION. Collected papers from scientific and technical conferences, symposia, seminars, or other meetings sponsored or cosponsored by NASA.

- SPECIAL PUBLICATION. Scientific, technical, or historical information from NASA programs, projects, and missions, often concerned with subjects having substantial public interest.

- TECHNICAL TRANSLATION. Englishlanguage translations of foreign scientific and technical material pertinent to NASA's mission.

Specialized services that complement the STI Program Office's diverse offerings include creating custom thesauri, building customized data bases, organizing and publishing research results ... even providing videos.

For more information about the NASA STI Program Office, see the following:

- Access the NASA STI Program Home Page at http://www.sti.nasa.gov

- E-mail your question via the Internet to help@sti.nasa.gov

- Fax your question to the NASA Access Help Desk at (301) 621-0134

- Telephone the NASA Access Help Desk at (301) 621-0390

- Write to: NASA Access Help Desk NASA Center for AeroSpace Information 7121 Standard Drive Hanover, MD 21076 
NASA/CR-2000-210062
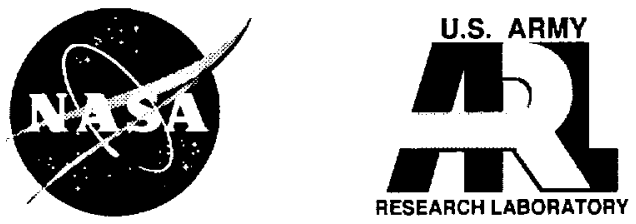

\section{Simulating Fatigue Crack Growth in Spiral Bevel Gears}

Lisa E. Spievak, Paul A. Wawrzynek, and Anthony R. Ingraffea Cornell University, Ithaca, New York

Prepared under Grant NAG3-1993

National Aeronautics and Space Administration

Glenn Research Center 


\section{Acknowledgments}

The research contained in this thesis was conducted under grant NAG3-1993 between Cornell University and

NASA Glenn Research Center. I wish to thank Dr. David Lewicki and Dr. Robert Handschuh of the U.S. Army Research Laboratory at NASA Glenn Research Center. Much of this thesis' work is a direct result of their advice and expertise. Lehigh University professor Dr. Eric Kaufmann's time and technical knowledge were instrumental with the scanning electron microscope observations contained in this thesis. In addition, Dr. Richard N. White at Cornell University volunteered his time and skills to photograph the tested spiral bevel pinion.

Many of his photographs are contained in this volume.

Available from

NASA Center for Aerospace Information 7121 Standard Drive

Hanover, MD 21076

Price Code: A06
National Technical Information Service 5285 Port Royal Road Springfield, VA 22100 Price Code: A06 


\section{TABLE OF CONTENTS}

CHAPTER ONE: INTRODUCTION......................................................... I

$1.1 \quad$ Background..........................................................................................

$1.2 \quad$ Numerical Analyses of Gears ........................................................................

1.3 Overview of Chapters ....................................................................................

CHAPTER TWO: GEAR GEOMETRY AND LOADING …………………...... 7

$2.1 \quad$ Introduction ................................................................................... 7

2.2 Basics of Spiral Bevel Gear Geometry ........................................................... 7

2.3 Teeth Contact and Loading of a Gear Tooth ...................................................11

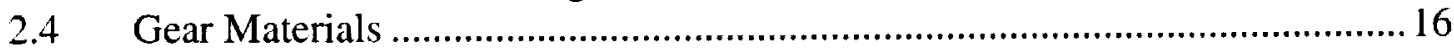

2.5 Motivation to Model Gear Failures ............................................................. 16

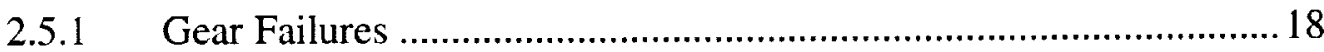

2.5.2 OH-58 Spiral Bevel Gear Design Objectives .................................... 19

2.6 Chapter Summary ...................................................................................... 19

CHAPTER THREE: COMPUTATIONAL FRACTURE MECHANICS …........21

$3.1 \quad$ Introduction .................................................................................21

3.2 Fracture Mechanics and Fatigue.....................................................................21

3.2.1 Fatigue ........................................................................................ 23

3.2.2 Example: Two dimensional, mode I dominant fatigue crack growth simulation with static, proportional loading........................................27

3.2.3 Example: Three dimensional, mode I dominant fatigue crack growth simulation with static, proportional loading......................................... 31

3.3 Fracture Mechanics Software ........................................................................33

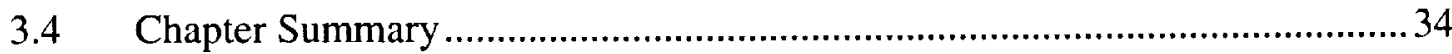

CHAPTER FOUR: FATIGUE CRACK GROWTH RATES................................35

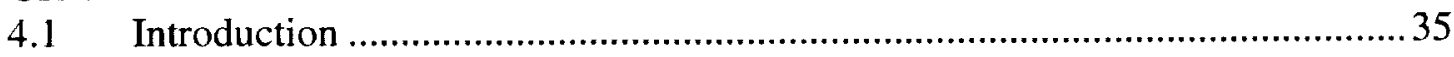

4.2 Fatigue Crack Closure Concept ........................................................................ 35

4.3 Application of Newman's Model to AISI 9310 Steel .......................................40

4.4 Sensitivity of Growth Rate to Low $R$ …………….......................................4

4.6 Chapter Summary .....................................................................................46

CHAPTER FIVE: PREDICTING FATIGUE CRACK GROWTH TRAJECTORIES IN THREE DIMENSIONS UNDER MOVING, NON-

PROPORTIONAL LOADS.........................................................................47

5.1 Introduction .................................................................................................

5.2 BEM Model ..........................................................................................

5.2.1 Loading Simplifications .................................................................49

5.2.2 Influence of Model Size on SIF Accuracy ………………………....51

5.3 Initial SIF History Under Moving Load ...........................................................54 
5.4 Method for Three Dimensional Fatigue Crack Growth Predictions Under Non-

Proportional Loading ..................................................................................5

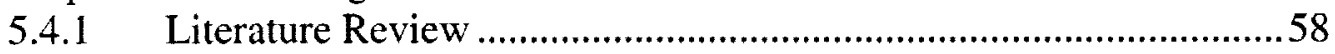

5.4.2 Proposed Method .............................................................................59

5.4.3 Approximations of Method ..............................................................6

5.5 Simulation Results.................................................................................64

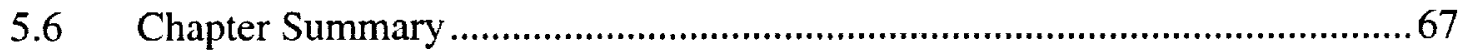

CHAPTER SIX: EXPERIMENTAL RESULTS..............................................69

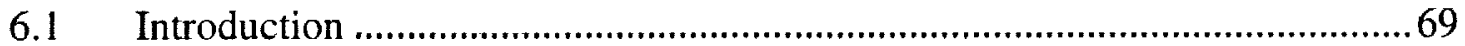

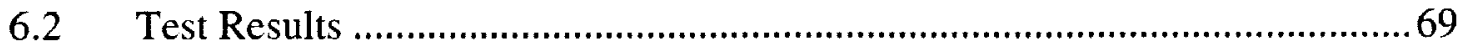

$6.3 \quad$ Fractography ......................................................................................... 71

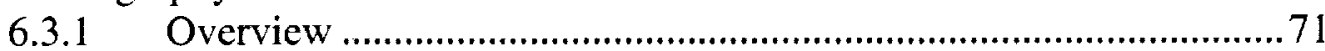

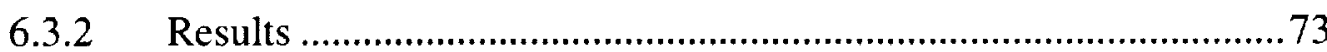

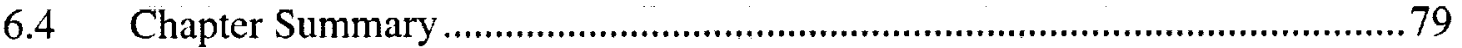

CHAPTER SEVEN: DISCUSSION AND SENSITIVITY STUDIES ....................81

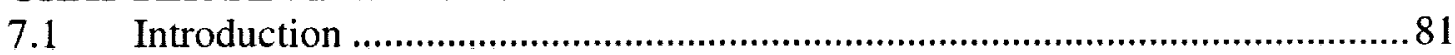

7.2 Comparisons of Crack Growth Results .......................................................... 81

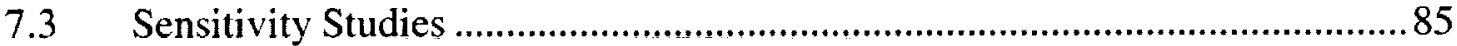

7.3.1 Fatigue Crack Growth Rate Model Parameters.................................8 86

7.3.2 Crack Closure Model Parameters ...................................................... 87

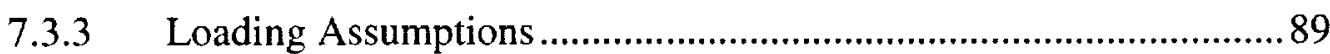

7.4 Highest Point of Single Tooth Contact (HPSTC) Analysis..............................96

7.5 Chapter Summary .................................................................................. 99

CHAPTER EIGHT: CONCLUDING REMARKS ……...................................101

8.1 Accomplishments and Significance of Thesis.............................................. 101

8.2 Recommendations for Future Research........................................................ 103

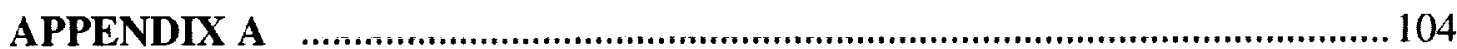

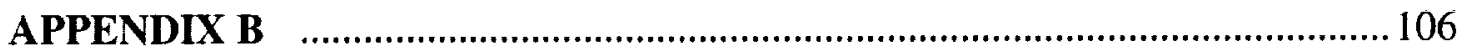

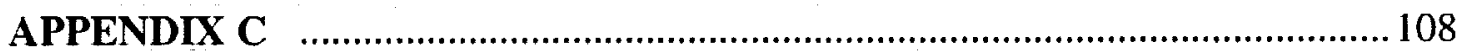

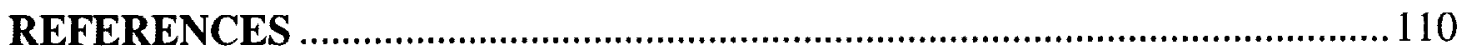




\section{LIST OF ABBREVIATIONS}

AGMA American Gear Manufacturers Association

BEM Boundary element method

EDM Electro-discharge machined

FEM Finite element method

FRANC3D FRacture ANalysis Code - 3D

HPSTC Highest point of single tooth contact

LEFM Linear elastic fracture mechanics

NASA/GRC National Aeronautics and Space Administration - Glenn Research Center

OSM Object Solid Modeler

RC Rockwell C

SEM Scanning electron microscope

SIF Stress intensity factor 


.




\section{CHAPTER ONE: \\ INTRODUCTION}

\subsection{Background}

A desirable objective in the design of aircraft components is to minimize the weight. A lighter aircraft operates more efficiently. A helicopter's transmission system is one example where design is focused on weight minimization. A transmission system utilizes various types of gears, such as spur gears and spiral bevel gears. Because spur gear geometry is relatively simple, optimizing the design of these gears using numerical methods has been researched significantly. However, the geometry of spiral bevel gears is much more complex, and less research has focused on using numerical methods to evaluate their design and safety.

One obvious method to minimize the weight of a gear is to reduce the amount of material. However, removing material can sacrifice the strength of the gear. In addition, fatigue cracks in gears are a design concern because of the cyclical loading on a gear tooth. Research shows that the size of a spur gear's rim with respect to its tooth height determines the crack trajectories [Lewicki et al. 1997a, 1997b]. This knowledge is critical because it allows the designer to predict failure modes based on geometry.

Two common failure modes of a gear are rim fracture and tooth fracture. Rim fracture, shown in Figure 1.1 [Albrecht 1988], can be catastrophic and lead to the loss of the aircraft and lives. On the other hand, Figure 1.2 is an example of a tooth fracture [Alban 1985]. Tooth fracture is the benign failure mode because it is most often detected prior to catastrophic failure. Knowing how crack trajectories are affected by design changes is important with respect to these two failure modes.
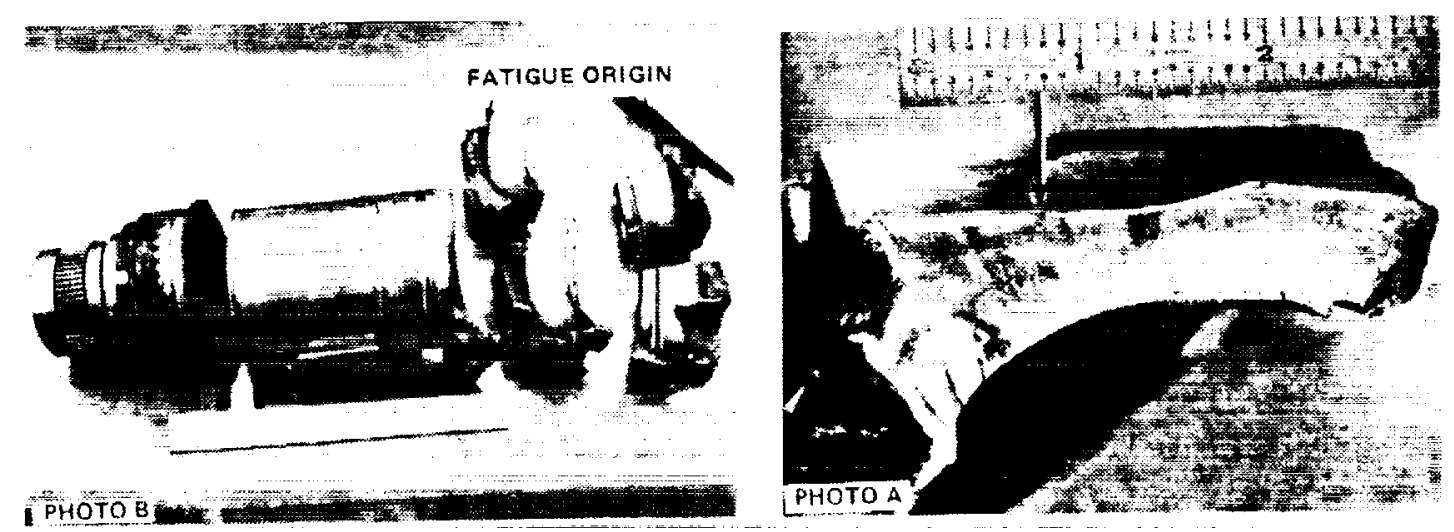

Figure 1.1: Spiral bevel gear rim failure [Albrecht1988]. 


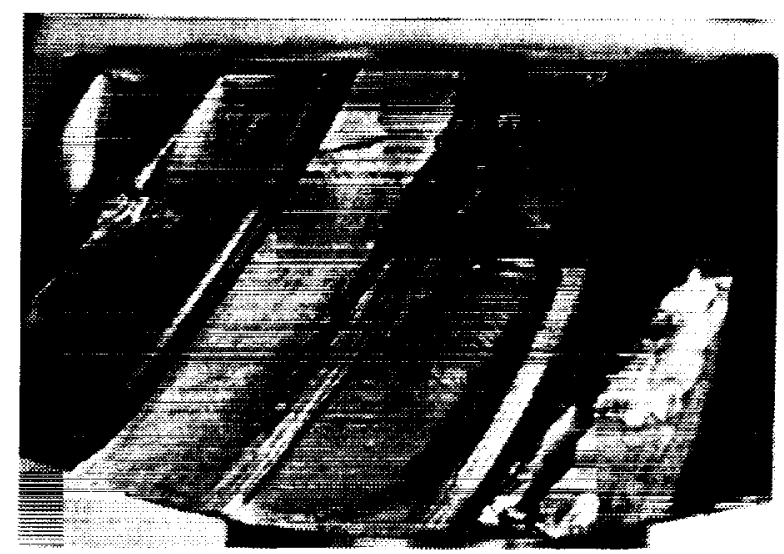

Figure 1.2: Spiral bevel gear tooth failure [Alban 1985].

In general, gears in rotorcraft applications are designed for infinite life; the gears are designed to prevent any type of failure from occurring. Developing a damage tolerant design approach could reduce cost and increase effectiveness of the gear. Lewicki et al.'s work on determining the effect of gear rim thickness on crack trajectories is a good example of how damage tolerance can be applied to gears. Knowing how the gear's geometry affects the failure mode allows a designer to select a geometry such that, if a crack were to develop, the failure mode would be benign. Other examples of damage tolerant design can be found in aircraft structures [Swift 1984] [Rudd 1984] [Miller et al. 1999], helicopter rotor heads [Irving et al. 1999], and train rails [Jeong et al. 1997].

Damage tolerance involves designing under the assumption that flaws exist in the structure [Rudd 1984]. The initial design then focuses on making the structure sufficiently tolerant to the flaws such that the structural integrity is not lost. Damage tolerant design allows for multiple load paths to prevent the structure from failing within a specified time after one element fails. In this regard, gears would be designed for the benign failure mode, tooth failure, as opposed to rim failure, which could be catastrophic.

Current American Gear Manufacturers Association (AGMA) standards use tables and indices to approximate the strength characteristics of gears [AGMA 1996]. The finite element method (FEM) and boundary element method (BEM) are becoming more useful and common approaches to study gear designs. A primary reason for this is the tremendous increase in computing power. Section 1.2 summarizes recent research related to modeling gears numerically.

Limited work has focused on predicting crack trajectories in spiral bevel gears. This is most likely because a spiral bevel gear's geometry is complex and requires a three dimensional representation. Structures with uncomplicated geometries, such as spur gears, can be modeled in two dimensions. Modeling an object in three dimensions requires a crack to also be modeled in three dimensions. Three dimensional crack representations introduce unique challenges that do not arise when modeling in two dimensions.

A three dimensional crack model consists of a continuous crack front. When a simpler geometry allows for a two dimensional simplification, a crack front is now 
represented by a single point, the crack tip. At a crack tip there are only two modes of displacement; in three dimensional models, however, there is a distribution of three modes of displacement along the crack front. Propagating a crack in two dimensions is completely defined by a single angle and extension length. On the other hand, along the crack front there is a distribution of angles and lengths.

Codes developed by the Cornell Fracture Group at Cornell University, such as Object Solid Modeler (OSM) and FRacture ANalysis Code - 3D (FRANC3D), have been developed to handle three dimensional fracture problems. FRANC3D explicitly models cracks and predicts crack trajectories under static loads. The crack growth models are based on accepted fatigue crack growth and linear elastic fracture mechanics (LEFM) mixed mode theories.

Because gears operate at high loading frequencies, the actual time from crack initiation to failure is limited. As a result, crack trajectories and preventing catastrophic failure modes are the primary concern in gear design. Crack growth rates are not as important. The goal of this research is to investigate issues related to predicting three dimensional fatigue crack growth in spiral bevel gears. A simulation that allows for arbitrarily shaped curved crack fronts and crack trajectories will be most accurate. In addition, the loading on a tooth as a function of time, position, and magnitude should be considered.

\subsection{Numerical Analyses of Gears}

Computational fracture mechanics applied to gear design is a relatively novel research area. As a result, the majority of work has been limited to two dimensional analyses. In three dimensions, very little work has predicted crack trajectories in gears. This section summarizes some pertinent developments in applying numerical methods and fracture mechanics to gear design.

The complexity of two dimensional gear analyses has evolved. Albrecht [1988] used the FEM to investigate gear tooth stresses, gear resonance, and transmission noise. Individual gear teeth were modeled in two dimensions and the increase in accuracy when using the FEM over AGMA standard indices for calculating gear tooth root stresses was demonstrated. Blarasin et al. [1997] used the FEM and weight function technique to evaluate stress intensity factors (SIFs) in specimens similar to spur gear teeth. Cracks with varying depths were introduced in two dimensional models and a constant single point load was applied. The SIFs were determined as a function of crack depth. Fatigue lives were calculated, but predictions of the crack trajectory were never performed. Flasker et al. [1993] used two dimensional FEM to analyze fatigue crack growth in a gear of a car gearbox. The analyses considered highest point of single tooth contact (HPSTC), but variable loading at that point. Residual stresses from the case and core were simulated with thermal loading. Based on a given load history, the crack was incrementally propagated. Lewicki et al. [1997a, 1997b] combined FEM and LEFM to investigate crack trajectories in thin rimmed spur gears. The work successfully matched crack trajectory predictions to experiments.

Limited three dimensional crack analyses of gears have been achieved. The work most often concerns simple geometries and loading conditions. Pehan et al. 
[1997] used the FEM to look at two and three dimensional spur gear models. Residual stresses due to case hardening were modeled as nodal thermal loads. Two different sized models were analyzed: one tooth including the arc length of the gear rim directly below the tooth and three teeth with the corresponding gear rim arc length. To determine the new crack front, they used a criterion such that the SIFs along the new front should be constant. Paris' model was used to calculate the fatigue lives based on the SIFs near the midpoint of the crack front. A constant load location with constant magnitude and simple spur gear geometry allowed Pehan et al. to consider only crack opening (mode $\mathrm{I}$ ) effects. Their method for determining the new crack fronts is computationally intensive and limited since three dimensional effects are not accommodated.

Lewicki et al. [1998] performed three dimensional crack propagation studies using the FEM and BEM to investigate fracture characteristics of a split tooth gear configuration. The geometry of the split tooth configuration is similar to a spur gear. The analyses used single load locations and explored propagation paths for various crack locations. The strong point of this work is that three dimensional simulations of crack trajectories were performed in addition to calculating fatigue crack growth rates.

Very little work, in addition to Lewicki et al.'s research, has used the BEM to analyze gears. Sfakiotakis [1997] performed two dimensional BEM analyses of gear teeth considering mechanical and thermal loads. Rather than perform trajectory predictions, they calculated SIFs for different size initial cracks with various loading conditions and crack locations. Fatigue loading was not considered. Fu et al. [1995] also used the BEM for stress analysis related to optimizing the forging die of spiral bevel gears.

The progression of research related to computer analysis of gears has led to the investigation of crack growth in spiral bevel gears. FEM models of spiral bevel gears can be created from Handschuh et al.'s [1991] computer program that models the cutting process of spiral bevel gears to determine tooth surface coordinates in three dimensions, Litvin et al. [1996] utilized this program, in conjunction with tooth contact analysis [Litvin et al. 1991], to determine how bearing (contact between mating gear teeth) changes with different spiral bevel gear tooth surface designs. Transmission error curves were generated that gave an indication of the efficiency of the gear.

Along with Litvin et al.'s work [1991], tooth contact analysis of mating gears has been explored by Bibel et al. [1995 and 1996], Savage et al. [1989], and Bingyuan et al. [1991]. Bibel et al. successfully modeled multi-tooth spiral bevel gears with deformable contact using the FEM. They conducted a stress analysis of mating spiral bevel gears and analytically modeled, using gap elements from general purpose finite element codes, the rolling contact between the gear teeth. Bibel et al.'s work can be used to investigate how changes in gear geometry affect tooth deflections. Variations in tooth deflections can alter the contact zone between gear teeth. Savage et al. developed analytical methods to predict, using tooth contact analysis, the shift in contact ellipses due to elastic deflections of a spiral bevel gear's shafts and bearings under loads. Savage et al. and Bibel et al.'s work was related to spiral bevel gears, however, they did not incorporate fracture mechanics. On the other hand, Bingyuan et 
al. approximated the geometry of gears in contact as a pair of disk rollers compressed together. The linear elastic stresses in the disks could be written in closed form. The SIFs were calculated using the closed form expressions. Bingyuan et al.'s primary focus was to calculate surface fatigue life and compute crack growth rates. No trajectory predictions were made.

The majority of the aforementioned research on spiral bevel gears is unrelated to failure, but rather associated with design and efficiency; methods have been developed to create numerical models of spiral bevel gears and predict contact areas. Crack trajectories have been predicted in gears with simpler geometry that can be represented by two dimensional models. This thesis is a natural extension of the research to date. The next step is to computationally model fatigue crack trajectories in spiral bevel gears.

\subsection{Overview of Chapters}

This thesis is divided into eight chapters. The first and last chapters are overview and summary. The remaining chapters each build upon one another and propose, apply, and evaluate methods for predicting fatigue crack growth in spiral bevel gears.

Chapter Two contains background information on gears, with particular attention to spiral bevel gears. The objective is to define vocabulary and concepts related to spiral bevel gears that will be used throughout the thesis. In addition, the work of the thesis is further motivated by examples of gear failures and the current design objectives for gears.

A focus of this thesis is to demonstrate that computational fracture mechanics can be used to analyze complex gear geometries under realistic loading conditions. LEFM and fatigue theories that are utilized to accomplish this task are presented in Chapter Three. Methods that are currently implemented in two and three dimensions to compute crack trajectories are demonstrated through examples.

Chapter Four explores the significance of compression loading on calculated crack growth rates. The magnitude of compressive stresses in a gear's tooth root is a function of the rim thickness. If fatigue crack growth rates are highly sensitive to this compression, then growth rates may warrant more attention in designing gears. The concept of fatigue crack closure is used to investigate fatigue crack propagation rates in AISI 9310, a common gear steel. First, the concept of fatigue crack closure is discussed. A material-independent method is presented for obtaining fatigue crack growth rate data that do not vary with stress ratio. The method is demonstrated using data at various stress ratios for pressure vessel steel. Next, the concepts are applied to AISI 9310 steel data to obtain an intrinsic fatigue crack growth model. This model is used to investigate the effect of low stress ratios on fatigue crack growth in AISI 9310.

Chapter Five is an initial investigation into predicting three dimensional fatigue crack trajectories in a spiral bevel pinion under a moving load. First, a boundary element model of a pinion is developed. A method to represent the moving contact area on a gear tooth is discussed. Next, studies are conducted to determine the smallest model that still accurately represents the operating conditions of the pinion. Once the model is defined, a crack is introduced into the model, and the initial stress 
intensity factor history under the moving load is calculated. A method to predict fatigue crack trajectories under the moving load is proposed. The method is then applied to predict fatigue crack growth trajectories and rates in a spiral bevel pinion.

Fatigue crack growth results from a spiral bevel pinion in operation are necessary to validate the predictions. The sponsor of the research efforts of this thesis, NASA-Glenn Research Center (NASA/GRC), provided a pinion that was tested in their gear test fixture. Notches were fabricated into several of the teeth's roots prior to beginning the test. The test data and crack growth results are presented in Chapter 6 . In addition, in an effort to obtain crack front shape and crack growth rate information, the fracture surfaces are observed with a scanning electron microscope, and the results are given in the chapter.

The crack trajectory and fatigue life results from the simulation and the tested pinion are compared in Chapter Seven. To gain insight into the discrepancies between the prediction and test, the influence of model parameter assumptions and loading simplifications on crack trajectories and calculated fatigue crack growth rates are studied. Next, the necessity of the moving, non-proportional load crack growth method is evaluated by comparing the results to predictions that assume proportional loading.

Finally, Chapter Eight summarizes the accomplishments of the work in the previous chapters. Implications of the research conducted and suggestions for future work are given. 


\section{CHAPTER TWO: GEAR GEOMETRY AND MODELING}

\subsection{Introduction}

Chapter Two covers the basic terms and geometry aspects of a spiral bevel gear. This terminology and background is essential to motivate the numerical simulations of this thesis. A gear's design and geometry can be quite complex; however, only the fundamentals are explained in this chapter.

\subsection{Basics of Spiral Bevel Gear Geometry}

Gears are used in machinery to transmit motion. Gears operate in pairs. The two mating gears have similar shapes. The smaller of the mating gears is called the pinion, and the larger the gear. Motion is transferred from one gear to another by successively engaging teeth.

There are various types of gears. The shape of the teeth and the angle at which the mating gears are mounted are a few of the distinguishing characteristics between the gear types. Gears with intersecting shafts are called bevel gears. The most common angle to mount bevel gears is $\theta=90^{\circ}$, although any intersecting angle could be used. A bevel gear's form is conical. For comparison, as illustrated in Figure 2.1, spur gears are cylindrical, and the shafts of the gears are parallel. The geometry of a spur gear can be almost fully illustrated in two dimensions. However, the conical shape of a bevel gear requires a three dimensional illustration. This two and three dimensional difference is where the complexity of the work contained in this thesis lies.

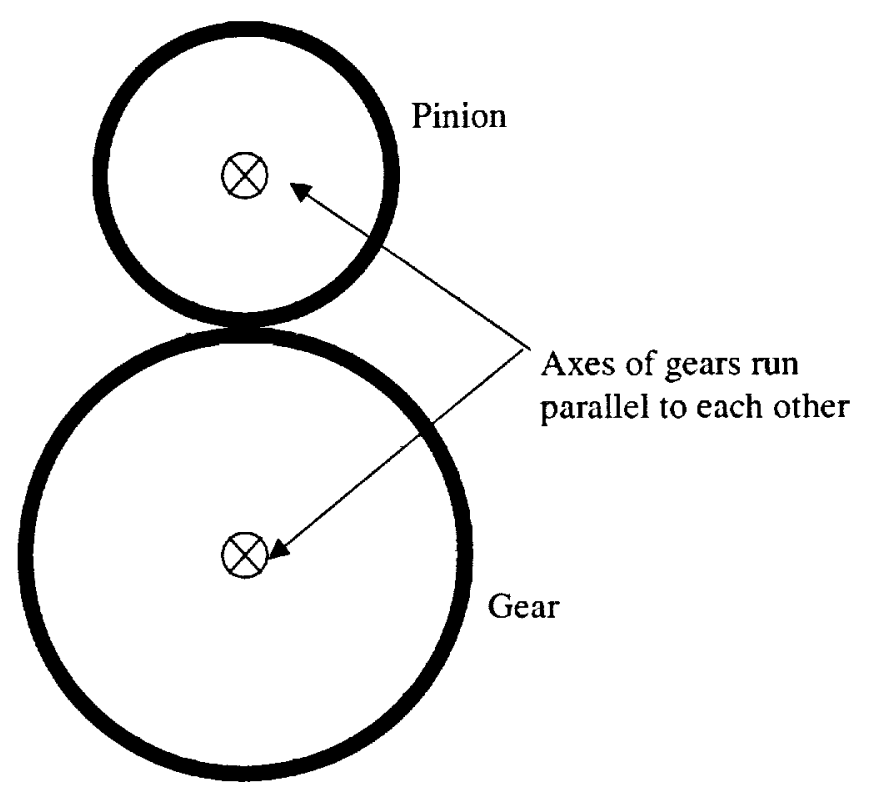

a) Spur gears operate with parallel axes 


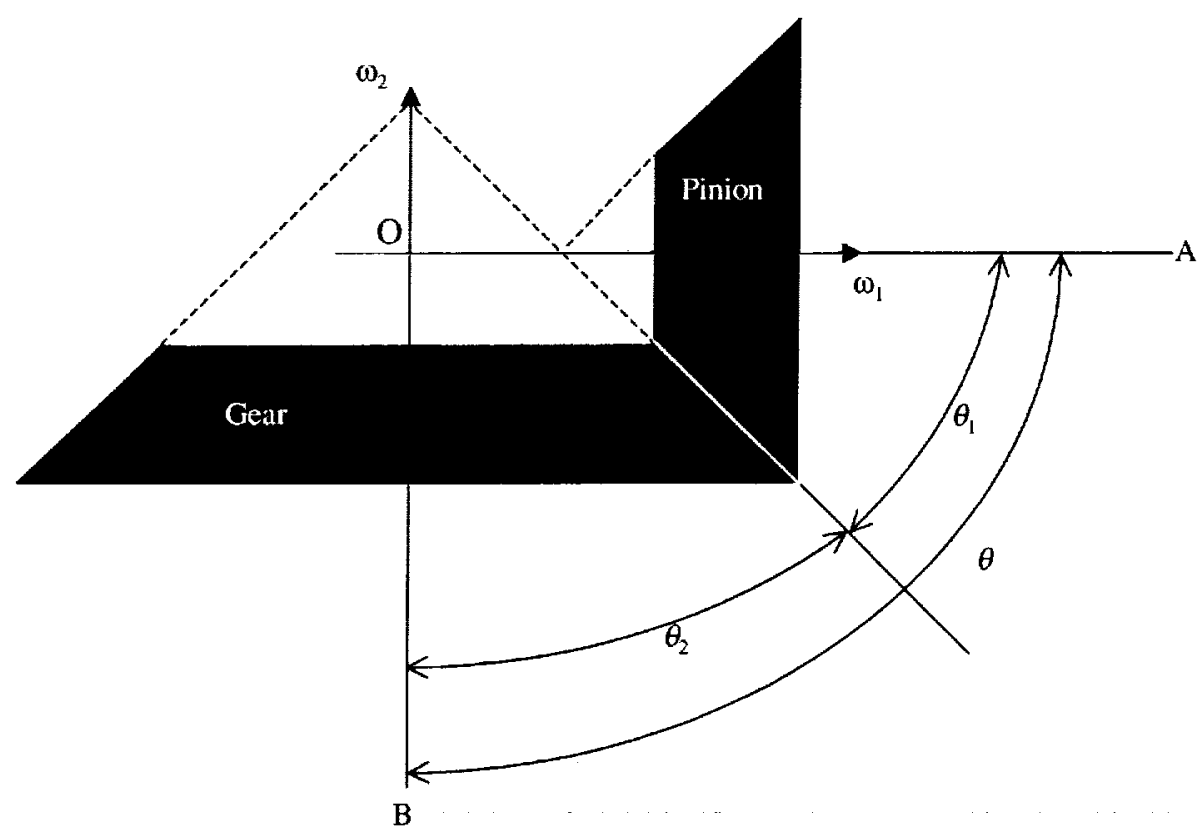

b) Bevel gears operate with intersecting axes

Figure 2.1: Schematics of spur (a) and bevel (b) gears.

The cone defined by the angle between a bevel gear's axis and the line of tangency with the mating gear is called the pitch cone. In Figure 2.1b, $\theta_{1}$ and $\theta_{2}$ define the pitch cones. The gear ratio is the ratio of the angular frequencies of the mating gears, $\omega_{2} / \omega_{1}$, which also equals the ratio of $\sin \left(\theta_{2}\right)$ to $\sin \left(\theta_{1}\right)$, or, due to geometry, the ratio of the number of gear teeth to the number of pinion teeth.

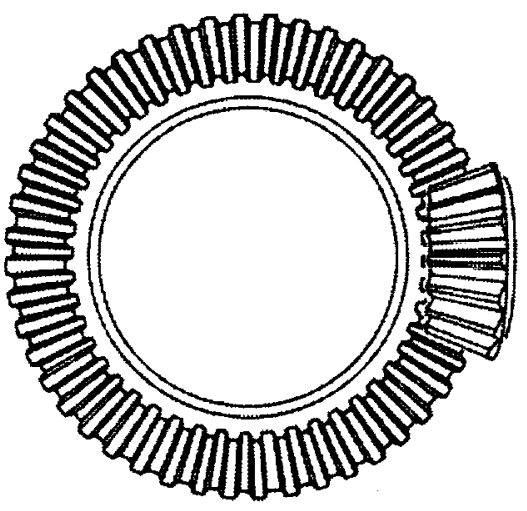

a) Straight bevel gear

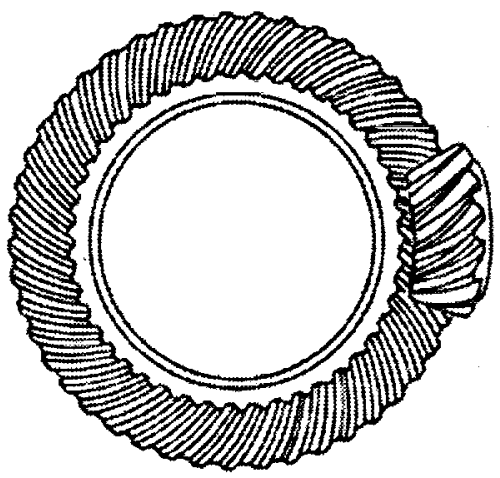

b) Spiral bevel gear

Figure 2.2: Bevel gear drawings [Coy et al. 1988].

Two common bevel gears are the straight bevel gear and the spiral bevel gear. The main difference between these two gears is the shape of their teeth. The teeth of the straight bevel gear are straight, and the teeth of the spiral bevel gear are curved. Figure 2.2 illustrates this difference. When looking along the axis of a spiral bevel gear, the teeth will either curve counterclockwise or clockwise, depending on whether 
the gear is left- or right-handed, respectively. So that the teeth can fit together, or mesh, a spiral bevel gear and pinion will always have opposite hands. The thickness and height of a spiral bevel gear tooth varies along the cone. The larger end of the tooth is the heel, and the smaller the toe. The curvature of the tooth creates concave and convex tooth surfaces on opposite sides of the tooth, Figure 2.3.

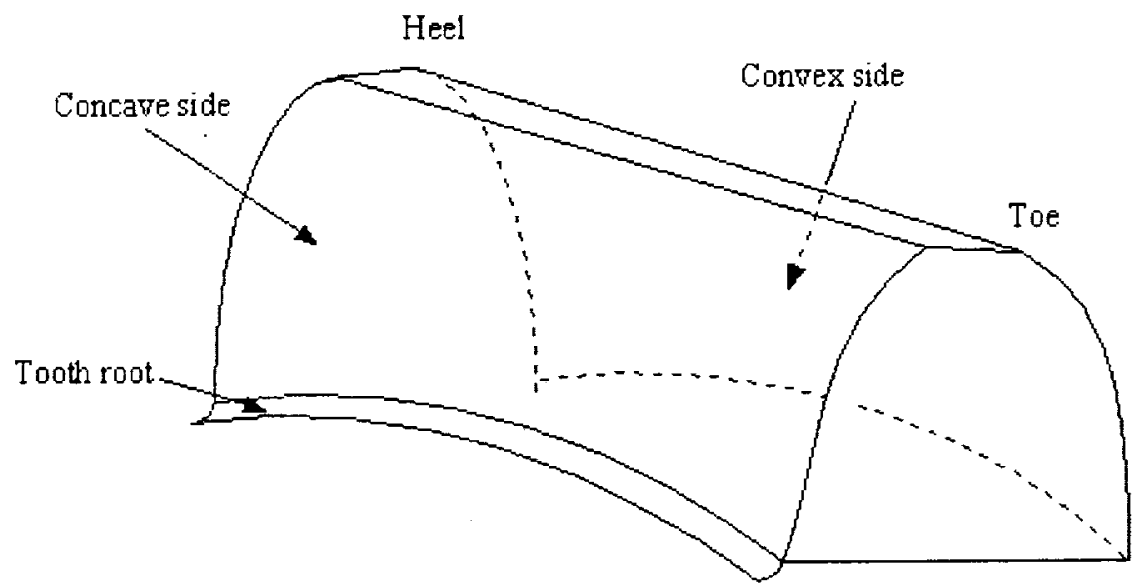

Figure 2.3: Schematic of a single spiral bevel gear tooth.

The tooth profile, as shown in Figure 2.4, is one side of the cross section of a gear tooth. The fillet curve is at the bottom of the tooth profile where it joins the space between the teeth. The region of the tooth near the fillet is the bottom land, and the area near the top of the profile is the top land.

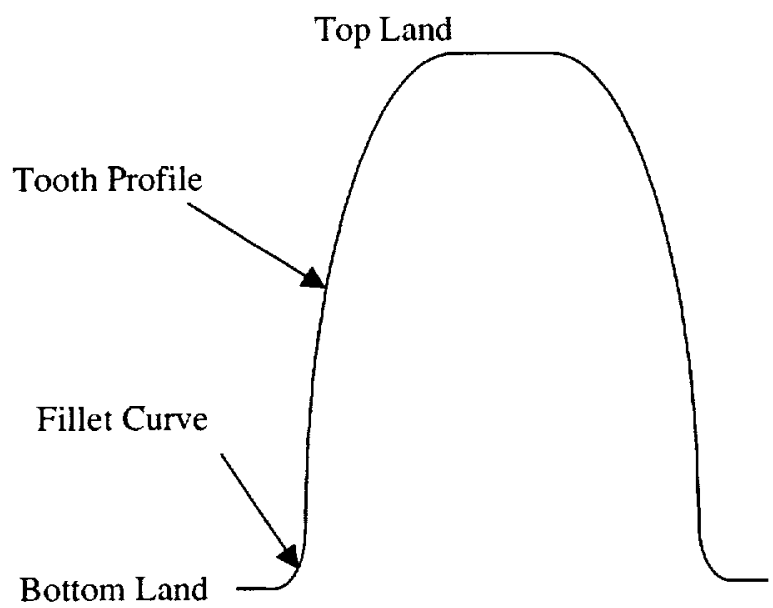

Figure 2.4: Schematic of cross section of a gear tooth.

The advantage of the spiral bevel gear's curved teeth is to allow for more than one tooth to be in contact at a time. This makes it significantly stronger than a straight bevel gear of equal size. Consequently, spiral bevel gears are commonly found in high speed and high force applications. One such application, which is the focus of this thesis, is in helicopter transmission systems. The mating spiral bevel gears in the 
transmission system convert the power from the horizontal engine shaft to the vertical shaft of the main rotor. Gears in this application typically operate at rotational speeds of $6000 \mathrm{rpm}$ and transmit on the order of $300 \mathrm{hp}$ of power.

Many parallel axis gears, such as spur gears, have involute tooth profiles. As sketched in Figure 2.5, the involute curve can be visualized by unwrapping thread from a spool while keeping the thread taut. The path traced by the end of the string is an involute curve. The spool is the evolute curve. All involute gear geometries are generated from circle evolute curves. The involute curve then becomes a spur gear tooth's profile. A closed form solution for the coordinates along the curve exists for this type of geometry. As a result, the tooth's surface coordinates can be calculated with relative ease. However, the geometry of a spiral bevel tooth is much more complex, and there is no closed form solution to describe the surface coordinates. Handschuh et al. [1991] developed a program to numerically calculate the surface coordinates of a spiral bevel gear tooth. The program models the kinetics of the cutting process in creating the gear, along with the basic gear geometry. The program calculates the coordinates of a spiral bevel gear tooth in three dimensions for use as input to a finite element model. The numerical models in this thesis were all created using the tooth geometry coordinates as defined by Handschuh et al.'s program.

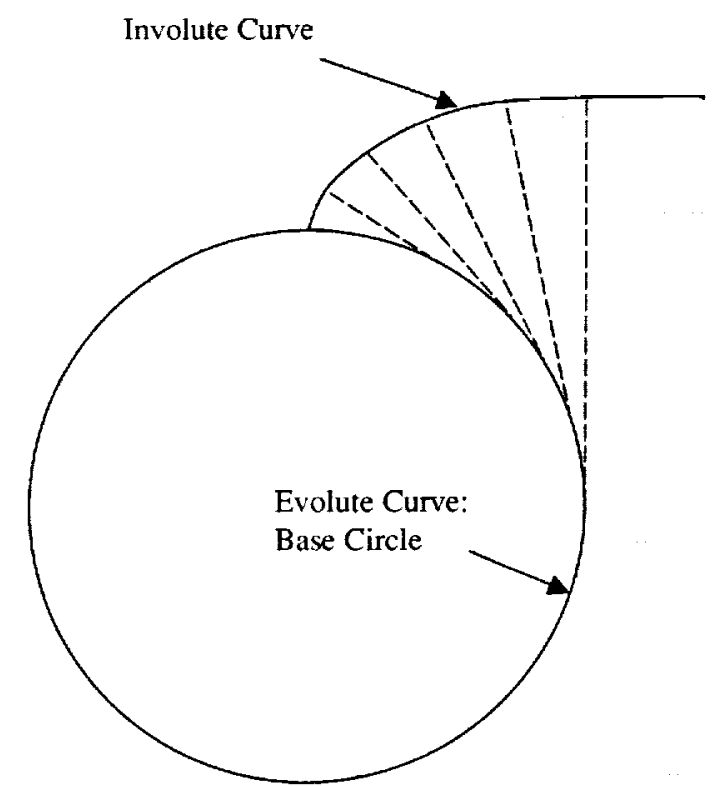

Figure 2.5: Generation of an involute curve. 


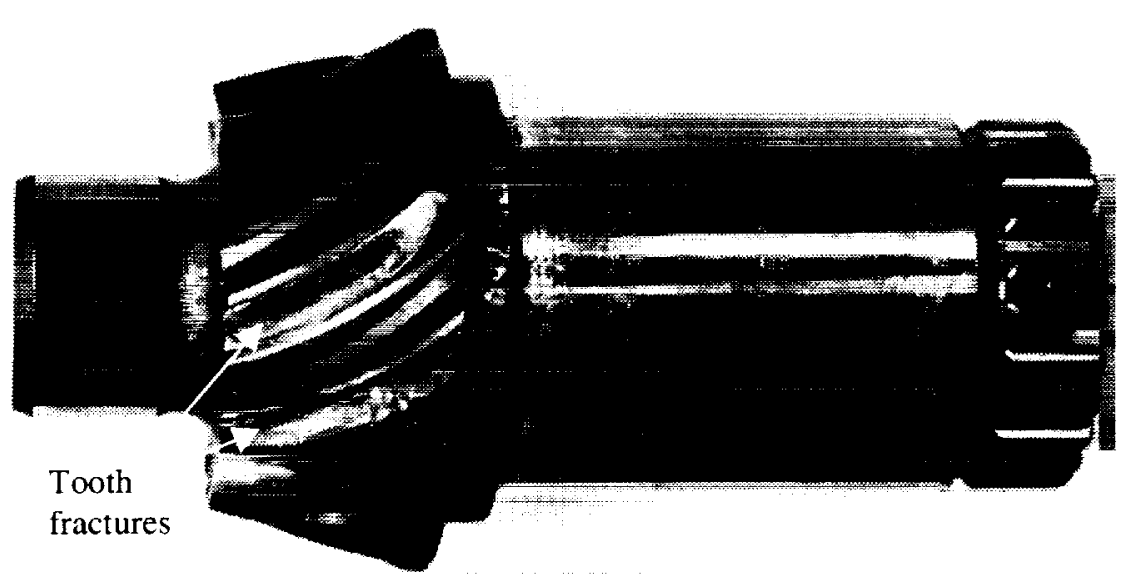

Figure 2.6: OH-58 spiral bevel pinion with two fractured teeth.

A spiral bevel gear set is used in the main rotor transmission of the U.S. Army's OH-58 Kiowa Helicopter. An OH-58 spiral bevel pinion that exhibited tooth fracture during an experiment is shown in Figure 2.6.

The geometry of the OH-58 gear set will be used throughout this thesis. In the set, a 19 tooth spiral bevel pinion meshes with a 71 tooth spiral bevel gear. The pinion's shafts are supported by ball bearings. The input torque is applied at the end of the pinion's large shaft. The approximate dimensions of a pinion tooth are given schematically in Figure 2.7.

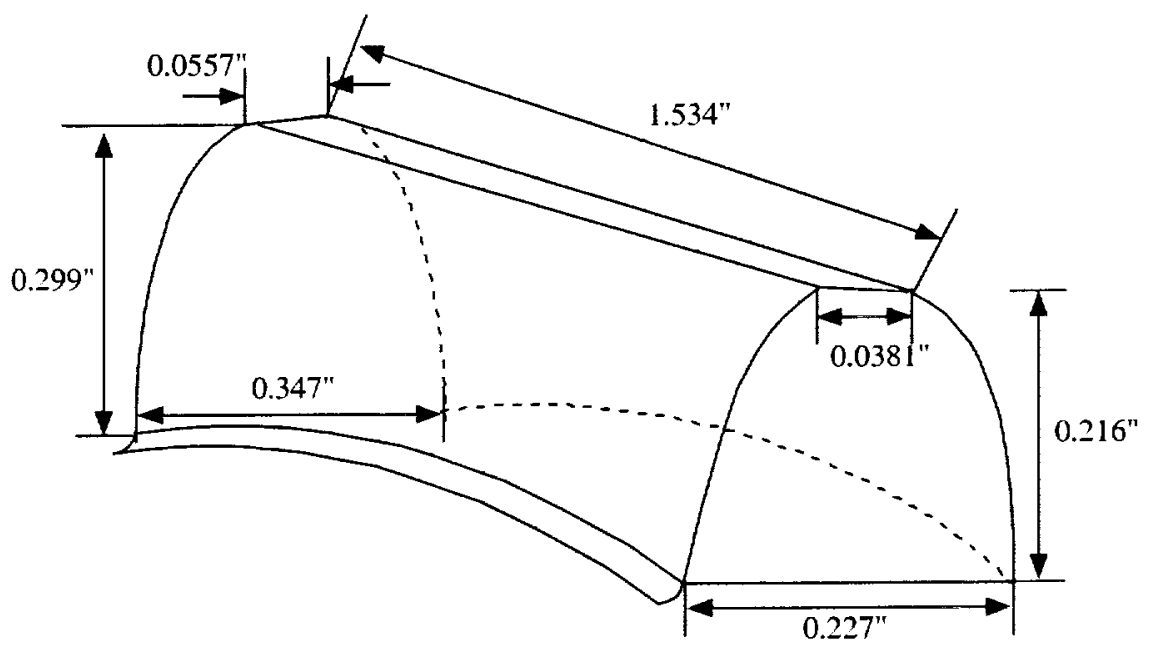

Figure 2.7: Approximate dimensions of $\mathrm{OH}-58$ spiral bevel pinion tooth.

\subsection{Teeth Contact and Loading of a Gear Tooth}

According to the theory of gears, there is a point of contact between a spiral bevel gear and pinion at any instant in time where their surfaces share a common normal vector. In reality, the tooth surfaces deform elastically under the contact. The deformation spreads the point of contact over a larger area. The larger area has traditionally been approximated using Hertzian contact theory. This contact is conventionally idealized to spread over an elliptical area [Johnson 1985]. The center 
of the ellipse is the mean contact point, which determines the contact ellipse's location on the tooth surface. The orientations of the ellipse's minor and major axes are defined by the tooth surface's geometry, curvature, and the alignment between the gear and pinion. The length of the axes is a function of the load. It can be shown that the ratio of the axes' lengths is constant and is not a function of the load. The form of the equations for the length of the ellipse's semi-major and semi-minor axes, $a$ and $b$, respectively, is [Johnson 1985] [Timoshenko et al. 1970]:

$$
\begin{aligned}
& a=f\left[\frac{3 \pi}{4}\right]^{1 / 3} \\
& b=g\left[\frac{3 \pi}{4}\right]^{1 / 3}
\end{aligned}
$$

where $f$ and $g$ are functions defined by the geometry. The magnitude of force, $P$, exerted on the tooth is proportional to the input torque level and gear geometry.

The meshing of the mating gear teeth is a continuous process. The position of the area of contact and magnitude of the force exerted between the teeth varies with time as the gear rotates. Figure 2.8 illustrates schematically the progression of the contact area along a tooth of a left-handed spiral bevel pinion. In the schematic, the continuous process has been discretized into a series of elliptical contact patches, or load step increments. The darkened arrow demonstrates the direction the load moves. The actual tooth contact pattern during operation is a function of the alignment of the gear and pinion.

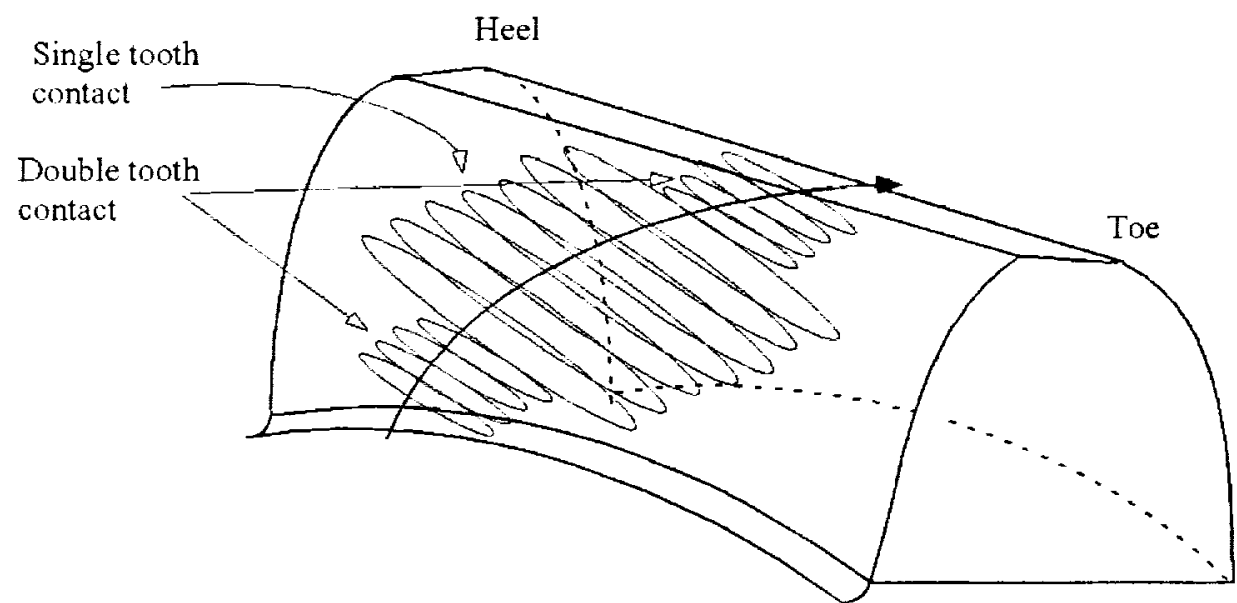

Figure 2.8: Schematic of tooth contact shape and direction during one load cycle of a left-handed spiral bevel pinion tooth.

Overlap in tooth contact between adjacent teeth results in two modes of contact: single tooth contact and double tooth contact. At the beginning of a meshing cycle for one tooth, two teeth of the pinion are in contact with the gear. As the pinion rotates, the adjoining tooth loses contact with the gear and only one pinion tooth receives all of the force. As the pinion continues to rotate, the load moves further up the pinion tooth, and the next pinion tooth comes into contact with the gear; the force on a pinion 
tooth is once again reduced due to the double tooth contact. The contact area will differ for single tooth and double tooth contact. The change in area of the contact is schematically illustrated in Figures 2.8 and 2.9.

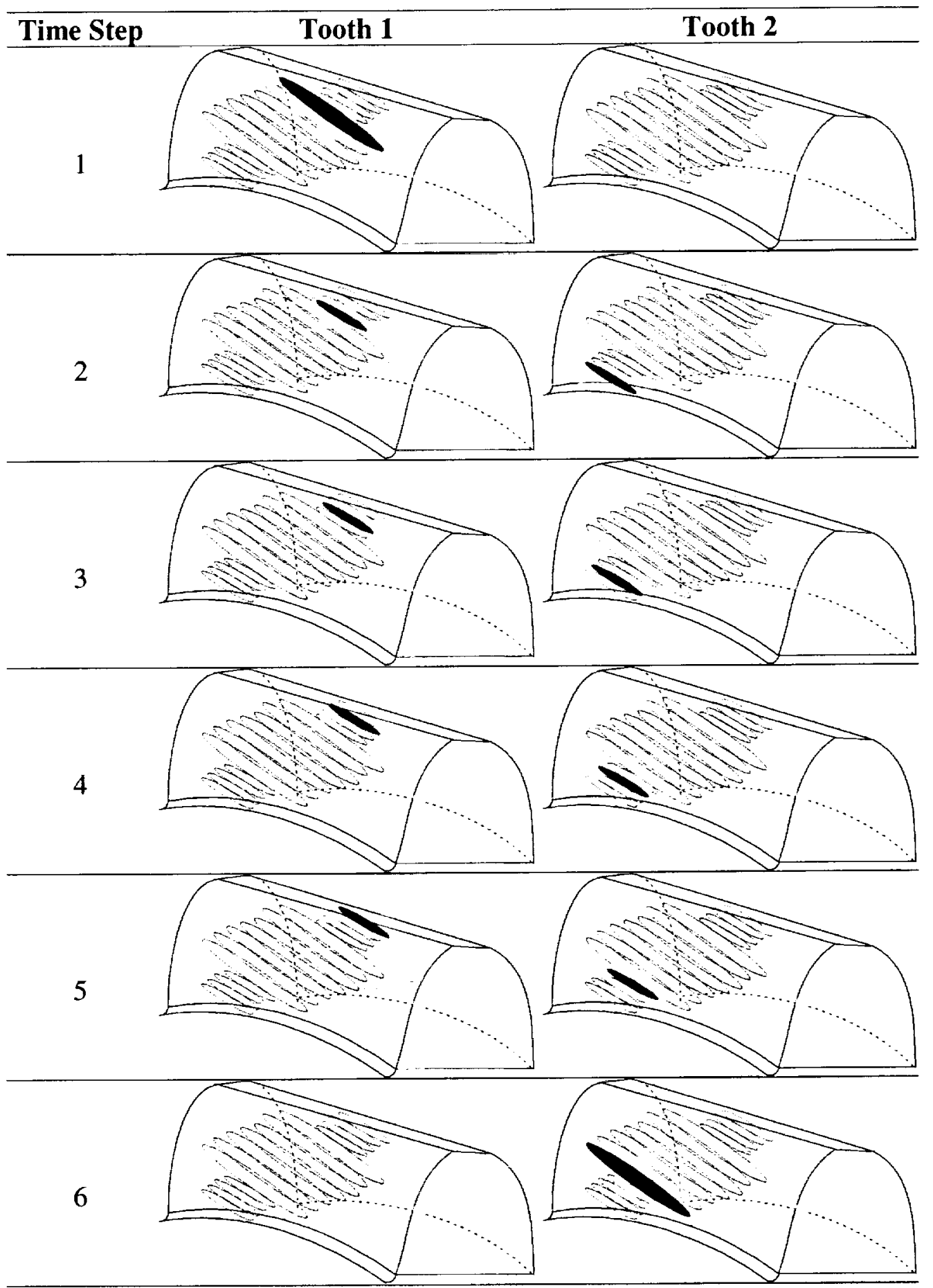

Figure 2.9: Schematic of load progression on adjacent pinion teeth. 
In Figure 2.9, tooth 1 and 2 are two adjacent teeth of a spiral bevel pinion. The ellipses represent "snap shot" areas of contact between a gear and a pinion's tooth. The darkened ellipse is the area that is currently in contact with the gear at a particular instant in time. Similar to Figure 2.8, the larger ellipses represent single tooth contact, and the smaller are areas of double tooth contact. The first row in Figure 2.9 begins with tooth 1 at the last moment of single tooth contact. After a discrete time step, the load on tooth 1 has progressed up the tooth and tooth 2 has come into contact near the root, as depicted in row two. In the final row, or time step, tooth 1 loses contact and tooth 2 advances into the stage of single tooth contact.

It is seen in Figures 2.8 and 2.9 that the contact area between mating spiral bevel gear teeth moves in three spatial dimensions during one load cycle. Most of the previous research into numerically calculating crack trajectories in gears has been performed on spur gears with two dimensional analyses and has not incorporated the moving load discussed above. Instead, a single load location on the spur gear tooth that produces the maximum stresses in the tooth root during the load cycle has been used to analyze the gear. This load position corresponds to the highest point of single tooth contact (HPSTC). Contact between spur gear teeth only moves in two directions, and, therefore, this simplification to investigate a spur gear under a fixed load at the HPSTC has proven successful [Lewicki 1995] [Lewicki et al. 1997a]. However, since the contact area between mating spiral bevel gear teeth moves in three dimensions, the crack front trajectories could be significantly influenced by this three dimensional effect. As a result, trajectories under the moving load should be predicted first and compared to trajectories considering only a fixed loading location at HPSTC. This approach is detailed in Chapters 5 and 7.

It has been implicitly assumed in the above discussion that the traction, or force over the contact area, is normal to the surface. Dike [1978] points out that this assumption is valid if there are no frictional forces in the contact area. He also states that is the case with gears since a lubricant is always used. The lubricant will make the magnitude of the frictional forces small compared to the normal forces. This assumption will be utilized in the numerical simulations.

In the same paper, Dike also asserts that there are two main areas in a gear tooth where the bending stresses may cause damage. The first is the location of maximum tensile stresses at the fillet of the tooth on the same side as the load. The second is at the fillet of the tooth on the side opposite the load, where the maximum compressive stresses occur.

This can be visualized by drawing an analogy between a cantilever beam and a gear tooth, Figure 2.10, Basic beam theory predicts that the maximum tensile stress occurs at the beam/wall connection on the outer most fibers on the same side as the applied load. The maximum compressive stress occurs at the same vertical location, on the side opposite the load. Similarly, as a gear tooth is loaded, it creates tensile stresses in the tooth root of the loaded side. In the root of the side opposite the load, there are compressive stresses. These compressive stresses might also extend into the fillet and root of the next tooth. 


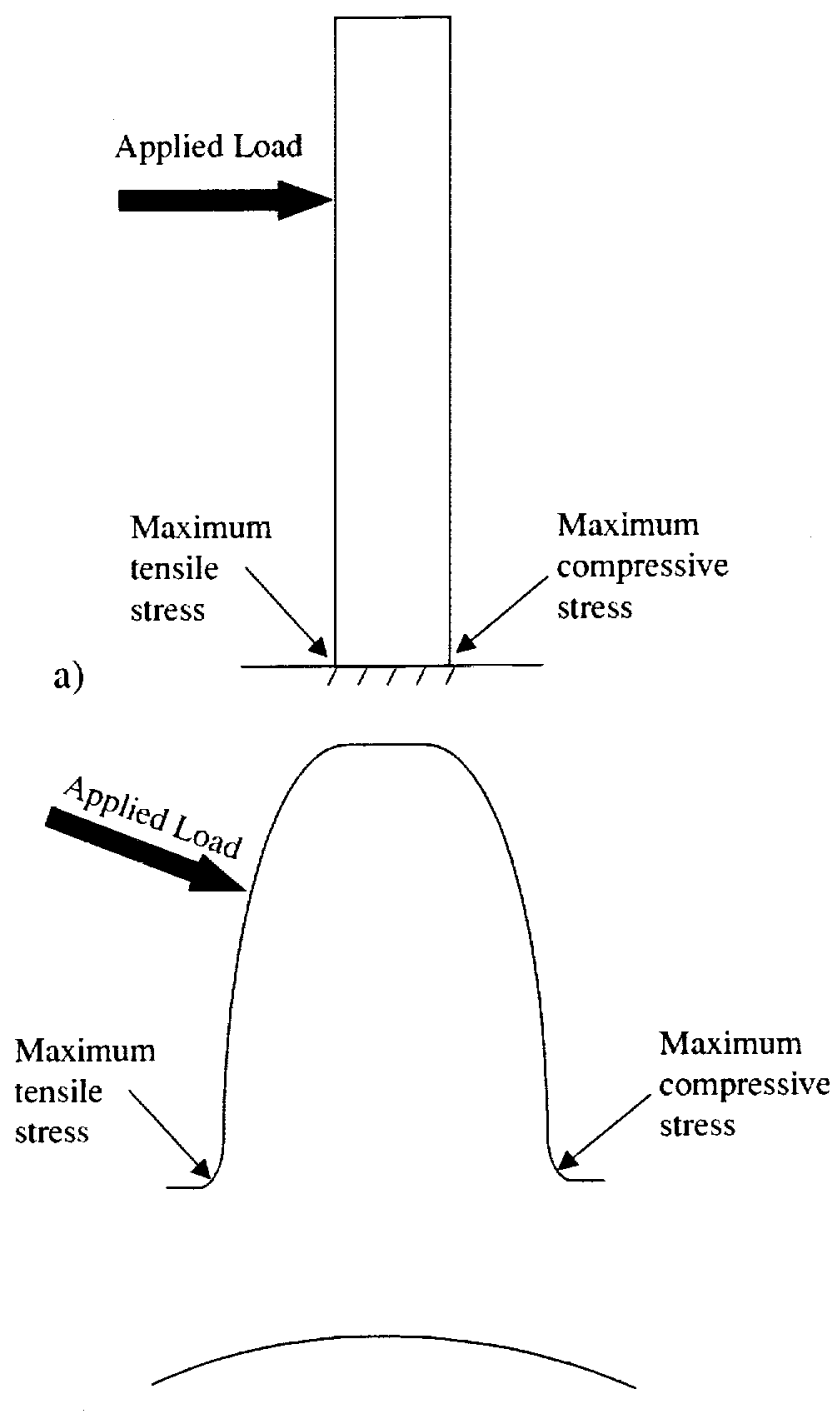

Figure 2.10: Stresses in cantilever beam (a) are analogous to gear tooth root (b).

The compressive stresses are noteworthy because Lewicki et al. [1997b] showed that the magnitude of the compressive stress increases as a gear's rim thickness decreases. The compressive stress could affect the crack propagation trajectories and crack growth rates. However, it is demonstrated in Chapter 4 that low stress ratios, i.e. large compressive stresses compared to tensile stresses, do not have a significant influence on crack predictions.

Up to this point, only frictional loads and traction normal to the tooth's surface have been discussed. The normal loads are the only loading conditions to be considered in this thesis. However, additional sources do produce forces on the gear. Some of these additional loads include dynamic effects, centrifugal forces, and residual stresses due to the case hardening of the gear. In addition, since a lubricant is always used when gears are in operation, lubricant could get inside a crack and create hydraulic pressure. 


\subsection{Gear Materials}

As discussed in Section 2.2, spiral bevel gears are commonly used in helicopter transmission systems. In this application, the gear's material impacts the life and performance of the gear. Most often a high hardenable iron or steel alloy is used. The traditional material for the OH-58 spiral bevel gear is AISI 9310 steel (AMS 6265 or AMS 6260). Some other aircraft quality gear steels are VASCO X-2, CBS 600, CBS 1000 , Super Nitroalloy, and EX-53. The choice of material is dependent on operating variables such as temperature, loads, lubricant, and cost. The material characteristics most important for gears are surface fatigue life, hardenability, fracture toughness, and yield strength. Table 2.1 shows the chemical composition of AISI 9310 [AMS 1996]. Table 2.2 contains relevant material properties.

Table 2.1: Chemical composition of AISI 9310 by weight percent [AMS 1996].

\begin{tabular}{|c|c|c|c|c|c|c|c|c|c|c|c|}
\hline & $\mathbf{C}$ & $\mathbf{M n}$ & $\mathbf{P}$ & $\mathbf{S}$ & $\mathbf{S i}$ & $\mathbf{C u}$ & $\mathbf{N i}$ & $\mathbf{C r}$ & $\mathbf{B}$ & $\mathbf{M o}$ & $\mathbf{F e}$ \\
\hline Minimum & 0.07 & 0.40 & -- & -- & 0.15 & -- & 3.00 & 1.00 & -- & 0.08 & 95.30 \\
\hline Maximum & 0.13 & 0.70 & 0.015 & 0.015 & 0.35 & 0.35 & 3.50 & 1.40 & 0.001 & 0.15 & 93.39 \\
\hline
\end{tabular}

Most gears are case hardened. Case hardening increases the wear life of the gear. In general, the gears are vacuum carburized to an effective case depth ${ }^{1}$ of 0.032 in 0.040 in $(0.813 \mathrm{~mm}-1.016 \mathrm{~mm})$. The case hardness specification is $60-63$ Rockwell $\mathrm{C}(\mathrm{RC})$, and the core hardness is 31 - $41 \mathrm{RC}$ [AGMA 1983].

Table 2.2: Material properties of AISI 9310.

\begin{tabular}{|l|c|}
\hline Tensile Strength $^{2}$ & $185 \times 10^{3} \mathrm{psi}$ \\
\hline Yield Strength $^{1}$ & $160 \times 10^{3} \mathrm{psi}$ \\
\hline Young's Modulus & $30 \times 10^{6} \mathrm{psi}$ \\
\hline Poisson's Ratio & 0.3 \\
\hline Fracture Toughness & $85 \mathrm{ksi}^{3}$ in $^{0.5}$ \\
\hline Average Grain Size $^{4}$ & $\begin{array}{c}\text { ASTM No. } 5 \text { or finer } \\
(\leq 0.00244 \mathrm{in})\end{array}$ \\
\hline
\end{tabular}

\subsection{Motivation to Model Gear Failures}

Gear failures can be categorized into several failure modes. Tooth bending, pitting, spalling, and thermal fatigue can all be placed in the category of fatigue failures. Examples of impact type of failures are tooth shear, tooth chipping, and case crushing. Wear and stress rupture are two additional modes of failure. According to [Dudley 1986], the three most common failures are tooth bending fatigue, tooth bending impact, and abrasive tooth wear. He gives examples of a variety of failures from tooth bending fatigue to spalling to rolling contact fatigue in both spur and spiral bevel gears.

\footnotetext{
${ }^{1}$ The effective case depth is defined as the depth to reach $50 \mathrm{RC}$.

${ }^{1}$ [Coy et al. 1995]

2 [Townsend et al. 1991]

${ }^{3}$ [AMS 1996]
} 
The focus of this thesis is on tooth bending fatigue failure because this is one of the most common failures. In general, tooth bending fatigue crack growth can lead to two types of failures. In rotorcraft applications, the type of failure could be either benign or catastrophic. Crack propagation that leads to the loss of one or more individual teeth will most likely be a benign type of failure. The remaining gear teeth will still be able to sustain load, and the failure should be detected due to excessive vibration and noise. On the other hand, a crack that propagates into and through the rim of the gear leaves the gear inoperable. The gear will no longer be able to carry any load, and will most likely lead to loss of aircraft and life.

Alban [1985, 1986] proposes a "classic tooth-bending fatigue" scenario. He suggests five conditions that characterize the "classic" failure:

1. The origin of the fracture is on the concave side in the root.

2. The origin is midway between the heel and the toe.

3. The crack propagates first slowly toward the zero-stress point in the root. As the crack grows, the location of the zero-stress point moves toward a point under the root of the convex side. The crack then progresses outward through the remaining ligament toward the convex side's root.

4. As the crack propagates, the tooth deflection increases only up to a point when the deflection is large enough that the load is picked up simultaneously by the next tooth. Since the load on the first tooth is relieved, the rate of increase in the crack growth rate decreases.

5. No material flaws are present.

Alban presents results from a photoelastic study of mating spur gear teeth. The study demonstrates the shift in the zero-stress point. The zero-stress point is where the tensile stresses in the root of loaded side of the tooth shift to compressive stresses on the load free side. Figure 2.11 shows stress contours for two mating spur gear teeth. In the bottom gear, one of the teeth is cracked and another tooth has already fractured off. The teeth of the top gear are not flawed. By comparing contours between the mating cracked and uncracked teeth, it is easy to pick out the zero-stress location shift toward the root of the load free side. The shift of the zero-stress location demonstrates the changing stress state in the tooth. This changing stress state drives the crack to turn. The point in the two dimensional cross section where the crack turns is actually a ridge when the third spatial dimension, the length of the tooth, is considered. This classic tooth failure scenario will be used as a guideline when evaluating the prediction and experimental results in the following chapters. 


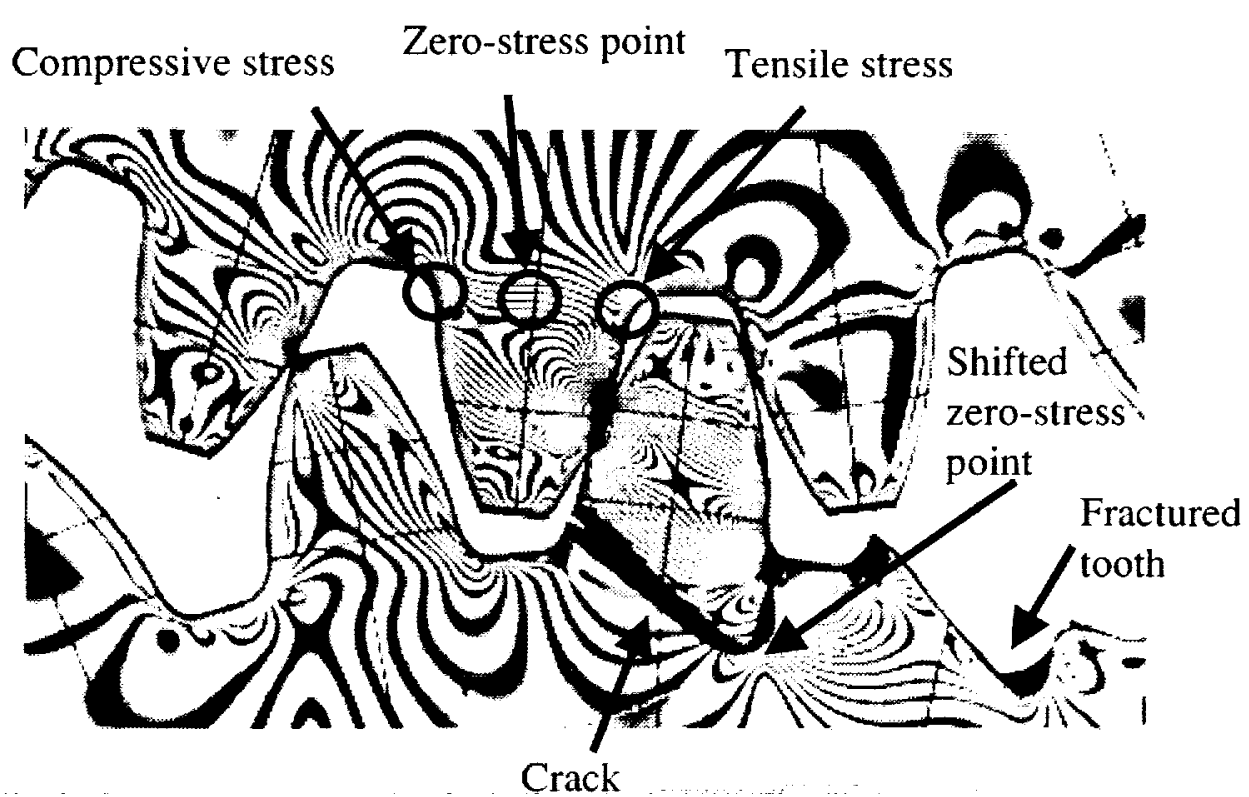

Figure 2.11: Photoelastic results from mating spur gear teeth (stress contour photograph from [Alban 1985]).

\subsubsection{Gear Failures}

Gears in rotorcraft applications are currently designed for infinite life. Therefore, gear failures are not common. However, failures do occur primarily as a result from manufacturing flaws, metallurgical flaws, and misalignment.

Dudley [1996] gives an overview of the various factors affecting a gear's life. Some of the more common metallurgical flaws listed are case depth too thin or too thick, grinding burns on the case, core hardness too low, inhomogeneities in the material microstructure, composition of the steel not within specification limits, and quenching cracks. In addition, examples of surface durability problems, such as pitting, are presented. A pitting flaw could develop into a starter crack for a fatigue failure.

Pepi [1996] examined a failed spiral bevel gear in an Army cargo helicopter. A grinding burn was determined as the origin of the fatigue crack. In addition, it was learned that the carburized case was deeper than acceptable limits in the area of the crack origin, which contributed to crack growth. Roth et al. [1992] determined a microstructure inhomogeneity, introduced during the remelting process, to be the cause of a fatigue crack in a carburized AISI 9310 spiral bevel gear. Both of these failures could be classified as manufacturing flaws.

Albrecht [1988] gives an example of a series of failures in the Boeing Chinook helicopter, which were caused by gear resonance with insufficient damping. Couchon et al. [1993] gives an example of a gear failure resulting from excessive misalignment. The excessive misalignment was due to a failed bearing that supported the pinion. The misalignment led to a fatigue crack on the loaded side of the tooth. An analysis of an input spiral bevel pinion fatigue crack failure in a Royal Australian Navy helicopter 
is given by McFadden [1985]. These examples demonstrate that gear failures do occur in service.

Gear experts are researching ways to make gears quieter and lighter through changes in the geometry. However, at the same time there is a tradeoff between weight, noise, and reliability. Geometry changes could have negative effects on the strength and crack trajectory characteristics of the gear. A design tool to predict the performance of proposed gear designs and changes, such as discussed by Lewicki [1995], would be extremely useful. Savage et al. [1992] used an optimization procedure to design spiral bevel gears using gear tooth bending strength and contact parameters as constraints. Including effects of geometry changes on the strength and failure modes could contribute greatly to his procedures.

\subsubsection{OH-58 Spiral Bevel Gear Design Objectives}

In rotorcraft applications, a primary source of vibration of the gear box is produced by the spiral bevel gears [Coy et al. 1987] [Lewicki et al. 1993]. In turn, the vibration of the gear box accounts for the majority of the interior cabin noise. As a result, recent design has focused on modifying the gear's geometry to reduce the vibration and noise. In addition, due to the application of the gear, a continuous design objective is to make the gear lighter and more reliable.

Adjusting the geometry of the gear, however, may jeopardize the gear's strength characteristics. Lewicki et al. [1997a] showed that the failure mode in spur gears is closely related to the gear's rim thickness. It was demonstrated that if an initial flaw exists in the root of a tooth, the crack would propagate either through the rim or through the tooth for a thin rimmed and thick rimmed gear respectively. As a result, a tool to evaluate the strength and fatigue life characteristics of proposed gear designs would be useful.

Albrecht [1988] demonstrated that AGMA standards to determine gear stresses and life were insufficient. He also showed the advantages of a numerical simulation method, such as the FEM, over the currently accepted AGMA standards at that time. The work of this thesis is an extension of the numerical approaches to determine gear stresses and life.

\subsection{Chapter Summary}

This chapter covered basic terminology and geometry aspects of gears. Concepts related to spiral bevel gears were the primary focus. In addition, methods to visualize and model the contact between mating spiral bevel gears were presented. Characteristics of a common gear steel, AISI 9310, were summarized. These materials properties will be used in the numerical simulations. Finally, some examples of gear failures and gear design objectives were discussed to motivate the significance of modeling gear failures numerically. 


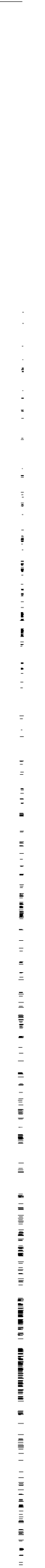




\section{CHAPTER THREE: COMPUTATIONAL FRACTURE MECHANICS}

\subsection{Introduction}

This chapter discusses areas of computational fracture mechanics relevant to the work of this thesis. The areas of focus are LEFM, fatigue, and the BEM. The $\mathrm{BEM}$ is used in a fashion similar to the more common FEM. The primary difference between the methods in three dimensional elasticity problems is that with the BEM only the surfaces, or boundaries, are meshed, as opposed to the volume that is meshed in the FEM. In computational LEFM, the displacement and/or stress results from a numerical analysis are used to calculate the SIFs. The SIFs are in turn used to predict how and where a crack may grow.

The analyses of this work are conducted using a suite of computational fracture mechanics programs developed by the Cornell Fracture Group. OSM is used to create a geometry model of the OH-58 spiral bevel pinion. FRANC3D is used as a pre- and post-processor to the boundary element solver program, BES. FRANC3D has built in features to compute SIFs using the displacement correlation technique.

\subsection{Fracture Mechanics and Fatigue}

Westergaard [1939], Irwin [1957], and Williams [1957] were the first to write closed form solutions for the stress distribution near a flaw. Their solutions were limited to very specific geometries and loading conditions. Their results, in the form of a series solution, showed that the stress a distance $r$ from a crack tip varied as $r^{-1 / 2}$. It can be shown that, under linear elastic conditions, the first term of the series solution for the stress near a flaw in any body, under mode $I$, or opening, loading is given by:

$$
\sigma_{i j}{ }^{(I)}=\frac{K_{I}}{\sqrt{2 \pi r}} f_{i j}^{(I)}(\theta)
$$

where $r$ and $\theta$ are polar coordinates as defined in Figure 3.1, $f_{i j}$ is a function of $\theta$ that is dependent on the mode of loading, and $K_{I}$ is the mode I stress intensity factor. The sub- and super-scripts $(I)$ denote mode I loading. Similarly, two other modes of loading can be defined as in-plane shear, mode II, and out-of-plane shear, mode III. The stress solutions for mode II and III loading are identical in form to Equation (3.1), but with all of the sub- and super-scripts $I$ replaced with $I I$ or $I I I$. 


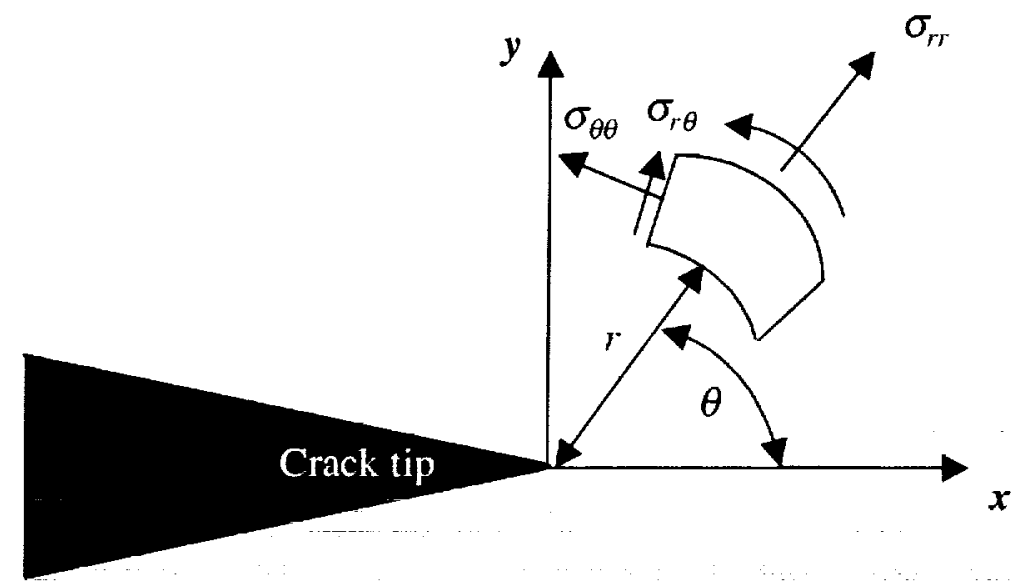

Figure 3.1: Coordinate system at a crack tip.

A significant feature of Equation (3.1) is that as $r$ goes to zero, or as one approaches the crack tip, this first term of the series solution approaches infinity. However, the higher order terms of the series will remain finite. For this reason, a large portion of LEFM focuses on this first term of the series expansion only. In reality, the stresses do not approach infinity at the crack tip. There is a zone around the tip where linear elastic conditions do not hold and plastic deformation takes place. This zone is called the plastic zone and results in blunting of the sharp crack tip. LEFM holds when the plastic zone is small in relation to the length scale of the crack.

The SIF is a convenient way to describe the stress and displacement distributions near a flaw in linear elastic bodies. The SIF for any mode is a function of geometry, crack length, and loading. The general equation for a SIF is

$$
K=\beta \sigma \sqrt{\pi a}
$$

$\beta$ is a dimensionless factor that depends on geometry, $2 a$ is the crack length, and $\sigma$ is the far field stress. It can be seen from Equation (3.2) that the units of $K$ are stress $* \sqrt{\text { length }}$.

For a crack to propagate, the energy supplied to the system must be greater than or equal to the energy necessary for new surface formation. When supplying energy to the system, the energy can primarily go into plastic deformation or new surface formation. LEFM assumes that all of the energy supplied goes into forming new surfaces. As a result, LEFM predicts the material at a crack tip will fail when the mode I SIF, $K_{I}$, reaches a critical intensity called the fracture toughness, $K_{I C}$. Fracture toughness is a material property and by definition is not dependent on geometry. Therefore, the criterion for fracture, or crack propagation, under LEFM, in mode I, is $K_{I} \geq K_{I C}$. Standard tests can be performed to measure values of fracture toughness [ASTM 1997]. The tests subject a standard specimen to pure mode I loading. The crack growth direction under pure mode I loading is self-similar. In other words, the crack tip in Figure 3.1 under only mode I loading will extend along the $x$-axis.

However, it is rare that a crack is subjected to pure mode I loading. More realistically, the loading will be a combination of all the modes. The mixed mode loading affects the fracture criterion and crack trajectory. For example, Mode II 
loading will turn, or kink, the crack away from self-similar crack propagation. There are several proposed methods to predict the direction of crack growth under mode I and II loading. The most widely accepted methods are the maximum principal stress theory [Erdogan et al. 1963], the maximum energy release rate theory [Nuismer 1975], and the minimum strain energy density theory [Sih 1974]. Due to ease of implementation and demonstrated accuracy, the maximum principal stress theory will be used in this thesis. The method is based on two assumptions. First, the crack will propagate radially from the crack tip. The second is that the crack will propagate in a direction that is perpendicular to the maximum tangential stress. In other words, the crack will kink at an angle $\theta_{m}$ where $\sigma_{\theta \theta}$ is a maximum. For mode I and II loading, assuming plane strain conditions, $\sigma_{\theta \theta}$ is

$$
\sigma_{\theta \theta}=\frac{1}{\sqrt{2 \pi}} \cos \frac{\theta}{2}\left[K_{l} \cos ^{2} \frac{\theta}{2}-\frac{3}{2} K_{I I} \sin \theta\right]
$$

The direction of crack growth can also be shown to correspond to the principal stress direction. Setting the partial derivative of $\sigma_{\theta \theta}$ with respect to $\theta$ equal to zero, the angle $\theta_{m}$ will be that which satisfies the equation

$$
K_{I} \sin \theta+K_{l l}(3 \cos \theta-1)=0
$$

From Equation (3.4), it is seen that if $K_{I I}$ equals zero, i.e. pure mode I loading, then the crack will propagate at an angle equal to zero. Figure 3.2 illustrates schematically the angle of crack trajectory, $\theta_{m}$, with respect to the crack front coordinate system.

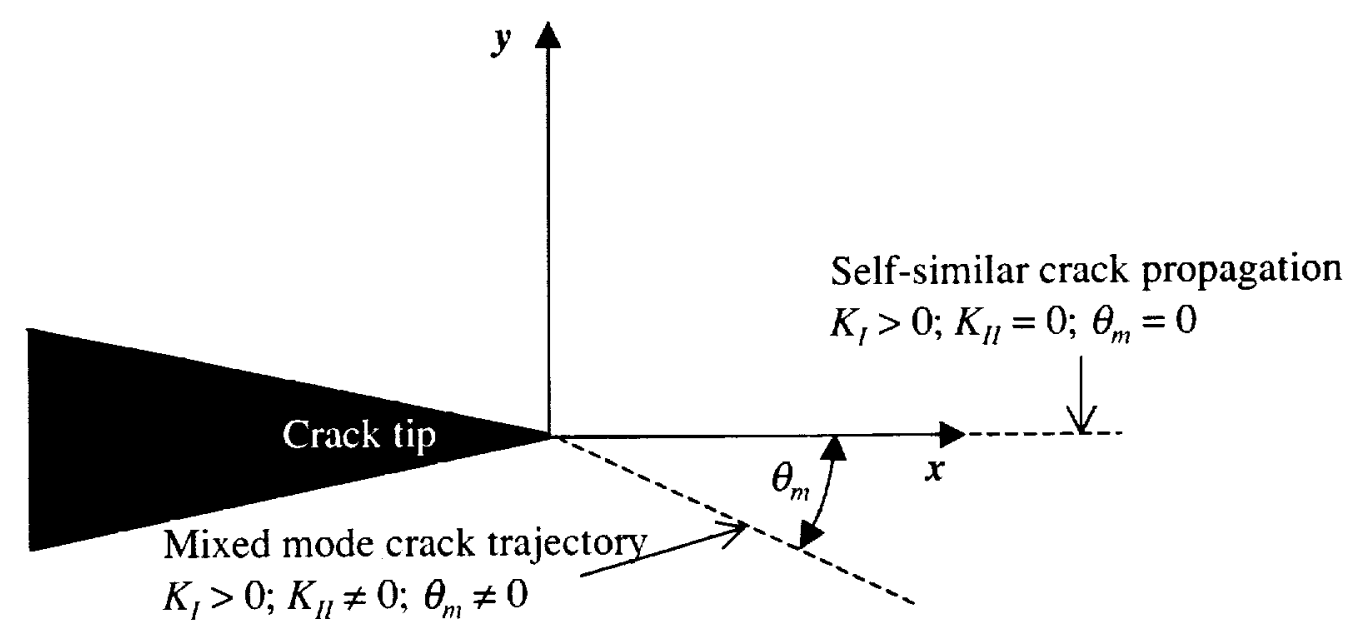

Figure 3.2: Angle of crack trajectory with respect to crack tip.

\subsubsection{Fatigue}

Cracks have been known to grow when the mode I SIF is less than $K_{I C}$. In these instances, the flaw has been subjected to cyclic loading. Cyclic loading can produce fatigue crack growth at loads significantly smaller than the fracture toughness of the material. Figure 3.3 illustrates how cyclic loading is characterized by the tensile load range, $\Delta S$, and the load ratio, $R . R$ is defined as the ratio of minimum stress, $S_{\text {min }}$, 
to maximum stress, $S_{\text {max. }}$, which, due to similitude, is equal to the ratio of minimum mode I SIF, $K_{\min }$, to maximum mode I SIF, $K_{\max }$.

$$
R=\frac{S_{\min }}{S_{\max }}=\frac{K_{\min }}{K_{\max }}
$$

Cyclic load histories can also be classified as proportional or non-proportional. When the ratio of $K_{I I}$ to $K_{I}$ is constant during the loading cycle, the loading is proportional. Non-proportional is the case when this ratio varies with time.

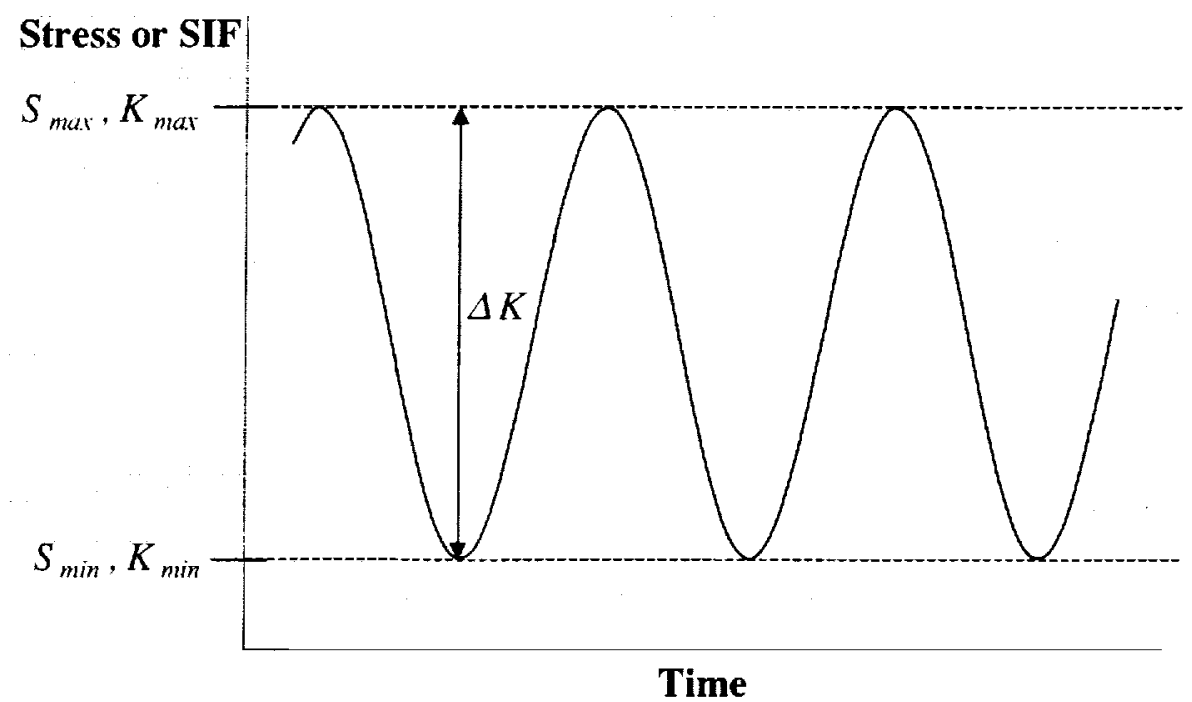

Figure 3.3: Cyclic load cycle.

There are three regimes of fatigue crack growth as demonstrated in Figure 3.4. Regime I is related to crack initiation and small crack effects. As noted on the plot, there is a threshold value, $\Delta K_{t h}$, below which fatigue crack growth will not occur. For AISI 9310 steel, values for $\Delta K_{\text {th }}$ are reported to range from approximately $3.5 \mathrm{ksi}^{*} \mathrm{in}^{0.5}$ $\sim 12 \mathrm{ksi}^{*} \mathrm{in}^{0.5}$ [Binder et al. 1980], [Forman ef al. 1984], [Proprietary source 1998]. As the stress ratio goes from positive to negative, the threshold value increases.

Regime II is commonly referred to as the Paris regime. The work of this thesis will only focus on crack growth in regime II. Crack initiation, small crack effects, and unstable crack growth (regime III) will be ignored. A seminal development in predicting fatigue crack growth was from [Paris et al. 1961] and [Paris et al. 1963]. They discovered that a crack grows in fatigue at a rate that is a function of $\Delta K_{l}$. They proposed that the nature of the curve in regime II could be described by:

$$
\frac{d a}{d N}=C\left(\Delta K_{l}\right)^{n}
$$

where $N$ is the number of cycles, and $C$ and $n$ were proposed as material constants. Equation (3.6) is commonly referred to as the Paris model. When the crack growth rate in regime $I I$ is plotted on a log-log scale as a function of $\Delta K$, the slope of the curve is $n$. If the curve is extrapolated to the vertical axis, the intercept is $C$. 
In regime $I I I$, the crack growth is unstable. A crack can grow in fatigue only when $K_{I}<K_{I C}$. As a result, regime III is bounded on the right by $\Delta K_{I C}$.

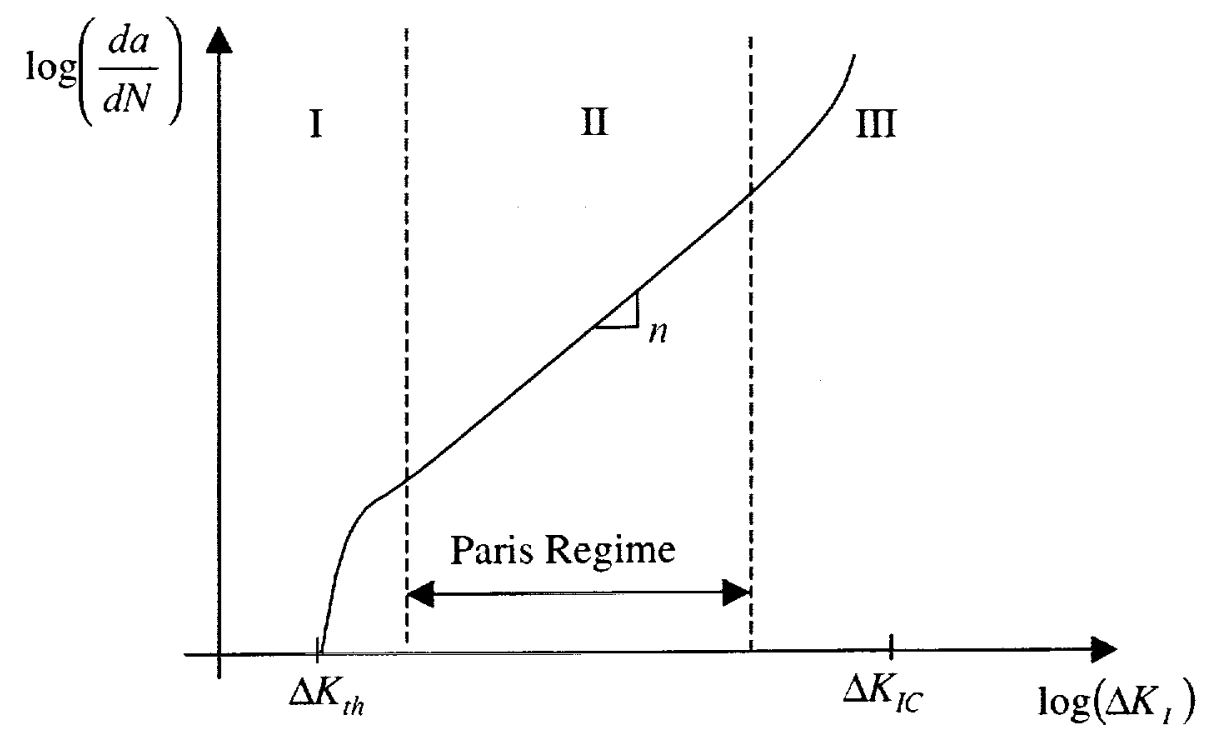

Figure 3.4: Typical shape of a fatigue crack growth rate plot.

Crack growth in regime II creates striations on the fracture surface in certain materials under appropriate loading conditions. It has been shown that the spacing between striations is roughly equal to the macroscopic crack growth rate $d a / d N$ [Forsyth 1962]. In general, ductile alloys, e.g. aluminum alloys, form the most well developed striations. The material of interest in this thesis, AISI 9310 steel, is capable of forming striations [Bhattacharyya et al. 1979] [Au et al. 1981] [McElvily et al. 1996]. Au et al. successfully correlated fatigue crack growth rates to fatigue striations in AISI 9310 steel.

Paris first proposed $C$ as a material property. However, experimental research has found that $C$ varies as a function of the stress ratio. The crack growth rate increases as the stress ratio increases. Fatigue crack growth data in regime $I I$ from tests conducted at different stress ratios, plots as shown in the left graph of Figure 3.5. The spread in the curves is explained by fatigue crack closure [Elber 1971]. In general, it has been found that a crack will prematurely close prior to the tensile load being entirely removed. The level of stress at which this premature closing occurs is $S_{o p}$ (or, due to similitude, $K_{o p}$ ). Incorporating fatigue crack closure phenomenon into Paris' model should collapse the curves into a single line (the right graph of Figure 3.5). This is accomplished by plotting on the abscissa $\Delta K_{\text {eff }}\left(\Delta K_{e f f}=K_{\text {max }}-K_{o p}\right)$, rather than $\Delta K$. This single curve is referred to as the "intrinsic" fatigue crack growth rate. More details of fatigue crack closure will be discussed in Chapter 4. 


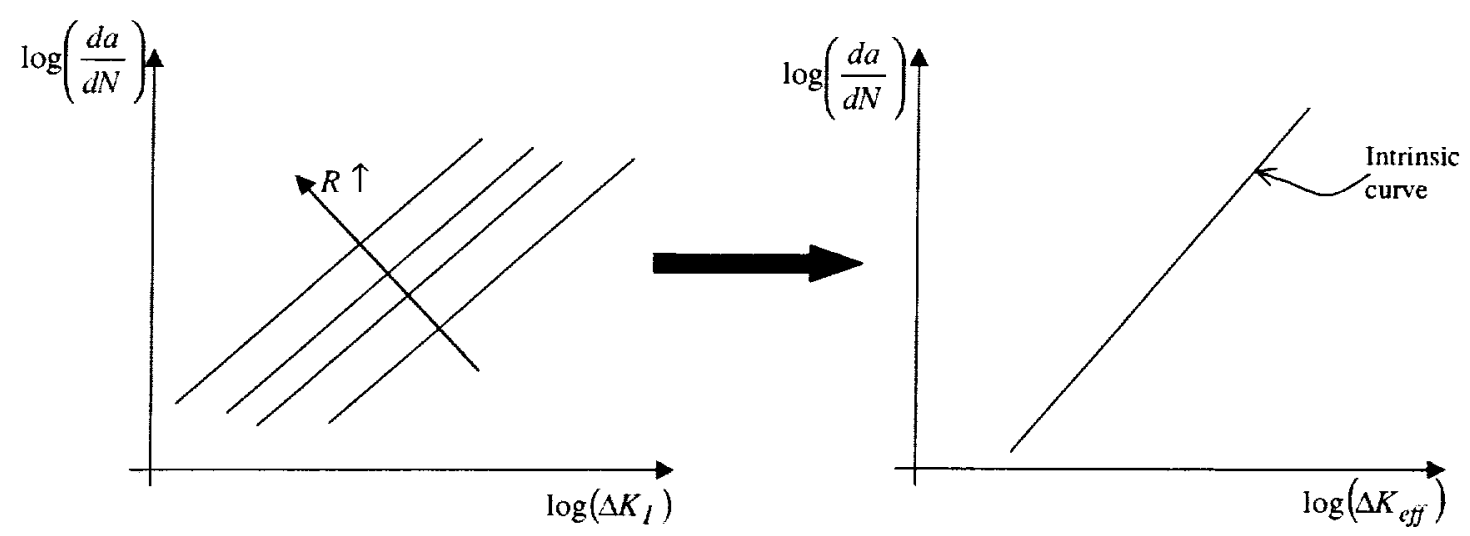

Figure 3.5: Schematic of fatigue crack growth rate data in Paris regime at different stress ratios collapsing into a single "intrinsic" curve.

Using Paris' model, the amount of crack growth per cycle for a given cracked object and load history can be predicted from the SIFs. In computational fracture mechanics, the FEM or BEM is used to calculate the SIFs. Several ways to calculate SIFs using numerical methods include the displacement correlation method [Chan et al. 1970], stiffness derivative [Parks 1974], $J$-integral [Rice 1968], and the universal crack closure integral [Singh et al. 1998]. The displacement correlation technique is used in this work because it relies only on displacement information on the boundary near the crack tip and because the method is computationally efficient. The numerical analyses of the spiral bevel pinion are conducted using the boundary element method, which solves for displacement information only on the boundaries. The displacement correlation method is computationally efficient since only a single numerical analysis is adequate to calculate the SIFs, unlike some of the other techniques that require two. Additionally, the mode I, II, and III SIFs are all calculated by the same method.

The displacement correlation method utilizes the fact that the displacements near a crack tip are proportional to the SIFs. Under pure mode I loading, the opening displacement, $u_{y}$, is given by [Owen et al. 1983]

$$
u_{y}=\frac{K_{l}}{4 \mu} \sqrt{\frac{r}{2 \pi}}\left[(2 \kappa+1) \sin \left(\frac{\theta}{2}\right)-\sin \left(\frac{3 \theta}{2}\right)\right]
$$

where

$$
\begin{aligned}
& \kappa=\frac{3-v}{1+v} \text { for plane stress } \\
& \kappa=3-4 v \text { for plane strain }
\end{aligned}
$$

$\mu$ is the shear modulus of the material, $v$ is Poisson's ratio, and $\theta$ is the angle between the location of the displacement and the normal to the crack tip. Equation (3.7) can be rearranged to solve for $K_{I}=f\left(u_{y}\right)$. Along the crack front $\theta=180^{\circ}$. Knowing the material properties ( $E$ (elastic modulus) and $v$ ), and the crack opening displacement $u_{y}$, at a given distance $r$ from the crack front, $K_{l}$ can be calculated.

$$
K_{l}=\frac{u_{,} E}{8\left(1-v^{2}\right)} \sqrt{\frac{2 \pi}{r}}
$$


Similarly, equations for $K_{I I}$ and $K_{I I I}$ can be written as a function of $u_{s}$, the displacement due to in plane shear, and $u_{=}$, the displacement due to out of plane shear, respectively. It is important to note that as $r$ approaches zero, the accuracy of the SIFs will decrease when using the displacement correlation method if the crack front elements are not capable of representing the singularities at the crack tip.

Crack growth rates are calculated from the SIF information and experimentally determined fatigue crack growth model parameters. The SIF information is also used to calculate the angle of propagation, e.g. Equation (3.4).

\subsubsection{Example: Two dimensional, mode I dominant fatigue crack growth simulation with static, proportional loading}

The purpose of this example is to demonstrate how fatigue crack predictions can be performed on a simple two dimensional model. The model assumptions are:

1. The location of applied load is not changing. This will be referred to as static loading.

2. The loading is proportional.

3. The crack growth can primarily be attributed to mode I opening. In other words, $K_{I} \gg K_{I I}$. This will be referred to as mode I dominant.

4. Crack closure effects will be ignored.

5. LEFM holds.

The method to predict crack trajectories in two dimensions is incremental. A series of finite element analyses are run which incrementally increase the crack length by a significant amount in relation to the model's geometry. For a given increase in crack length, the number of cycles to achieve that amount of growth can be calculated. For a given propagation step $i$, there are $N_{i}$ load cycles associated with it.

The amount of crack growth for one cycle is calculated as a function of the maximum stress in the load cycle. Because it is assumed the loading is proportional, it is straightforward to calculate the direction the crack will grow during the cycle using the maximum principal stress theory. However, there is no proposed method to calculate the final amount and direction of crack growth during one load cycle if the ratio $K_{I I} / K_{l}$ varies during the cycle, i.e. non-proportional loading. 


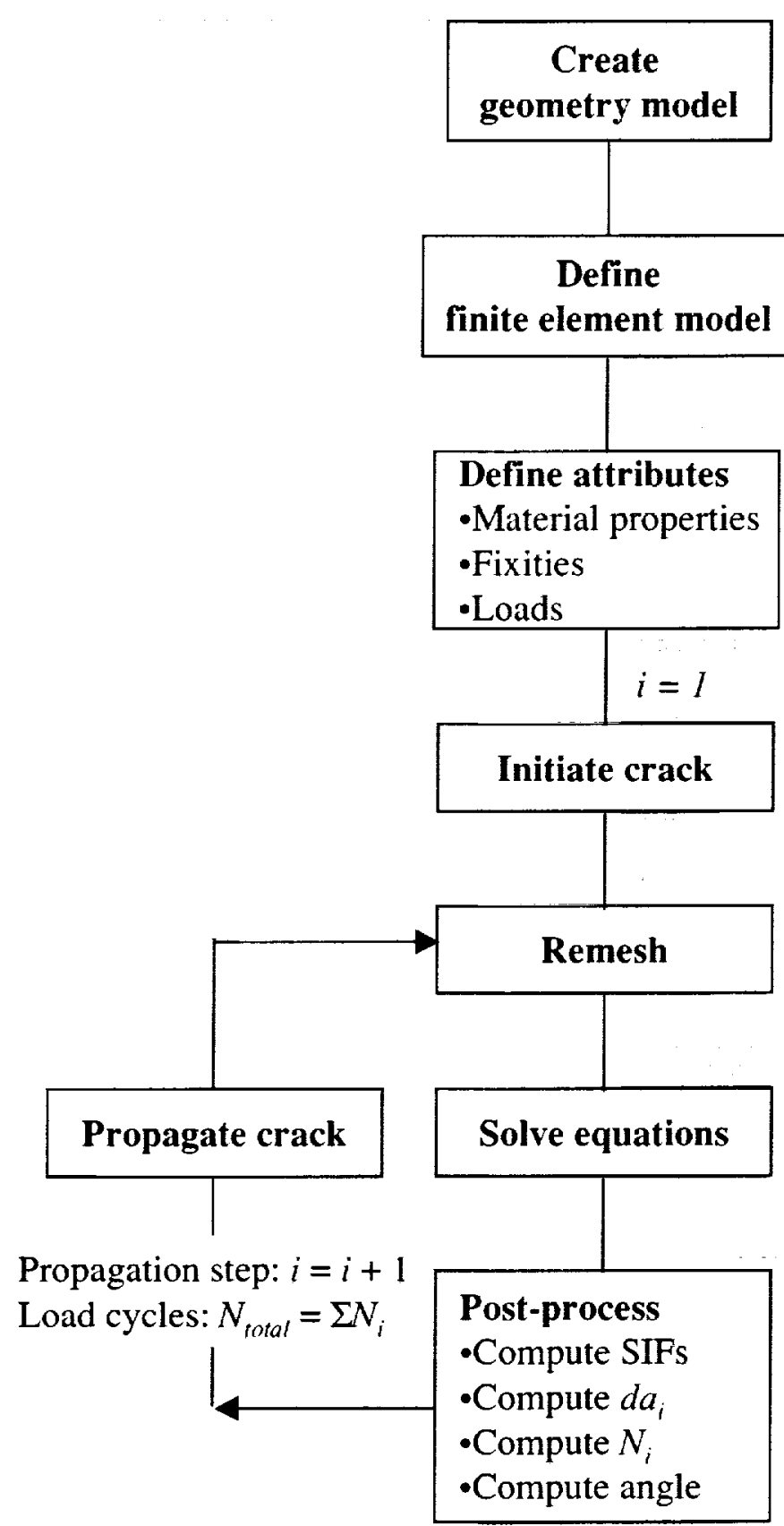

Figure 3.6: Flow chart of process to predict fatigue crack trajectory.

As outlined in Figure 3.6, the process begins with a geometry model. The geometry model is then discretized into a finite element mesh. Figure 3.7 shows the finite element mesh for an arbitrary geometry model that will be used for demonstrative purposes. This particular initial mesh consists entirely of quadratic eight-noded elements.

Model attributes must be defined next. The material properties are specified within the finite element program as a Young's Modulus of 29,000 ksi and Poisson's ratio of 0.25 . The thickness of the model is taken to be 1 inch. Boundary conditions 
must also be defined. A cyclic loading history like that shown in Figure 3.3 is assumed. The minimum applied traction is assumed to be zero, and the maximum applied traction is $S_{\text {max }}=100 \mathrm{ksi}$. A tensile traction is applied normal to the top edge. All of the nodes along the bottom edge are restrained in the vertical direction, and the far right corner node is restrained in the horizontal direction. If desired, at this stage the finite element solver could be run to calculate displacements, strains, and stresses in the uncracked geometry.

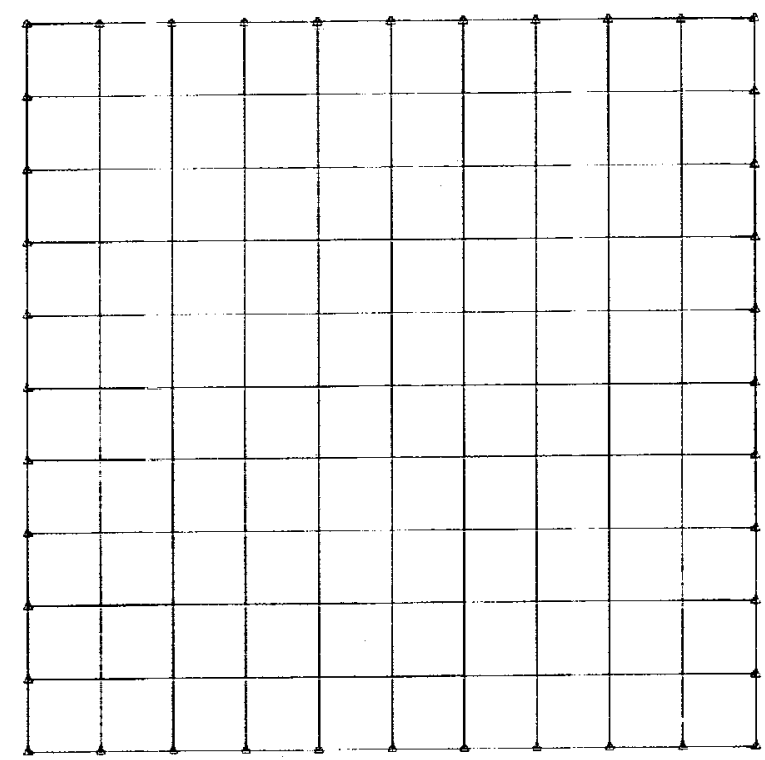

Figure 3.7: Two dimensional finite element model.

Next, a crack is introduced into the geometry model. With the change in geometry, the model must be remeshed. However, the damage to the mesh model is localized, and, therefore, only a small region around the crack must be remeshed. The mesh around the crack tip is a rosette of eight triangular, six-noded quarter point elements, Figure 3.8a. The remaining area is meshed with quadratic six-noded elements. Figure $3.8 \mathrm{~b}$ shows the initial edge crack and locally remeshed region. The boundary conditions, material properties, and loads were defined earlier and do not need to be redefined. At this point, displacements, strains, and stresses are solved for in the cracked geometry.

A method, such as the displacement correlation technique, is used to compute the stress intensity factors at the crack tip based on the relative displacements of the crack faces. Once the SIFs are calculated, Paris' model (Equation (3.6)) can be used to calculate the amount of crack growth for one load cycle, da/dN. A method, e.g. maximum principal stress (Equation (3.4)), is used to determine the direction of crack growth from the calculated SIFs. In most cases, the amount of crack growth for one load cycle will be on the order of $10^{-6}-10^{-4}$ inches. Since this is significantly smaller than the geometry features of the gear, it would be inefficient to update the geometry model for every load cycle. Consequently, a number of load cycles is assumed, e.g. $N_{i}=2,000$ cycles. Finally, the crack in the geometry model is extended by an amount 
$d a_{i}=(d a / d N)_{i} * N_{i}$, at an angle $\theta_{m}$ with respect to the self-similar crack trajectory (Figure 3.2). Again, the model must be remeshed locally, and the process is repeated.

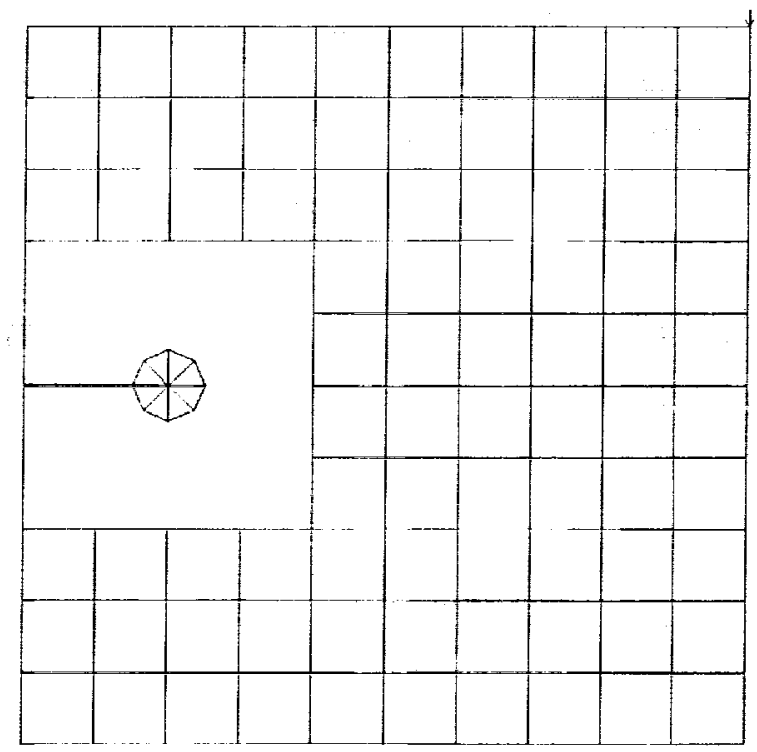

a) Initial crack and quarter point element rosette

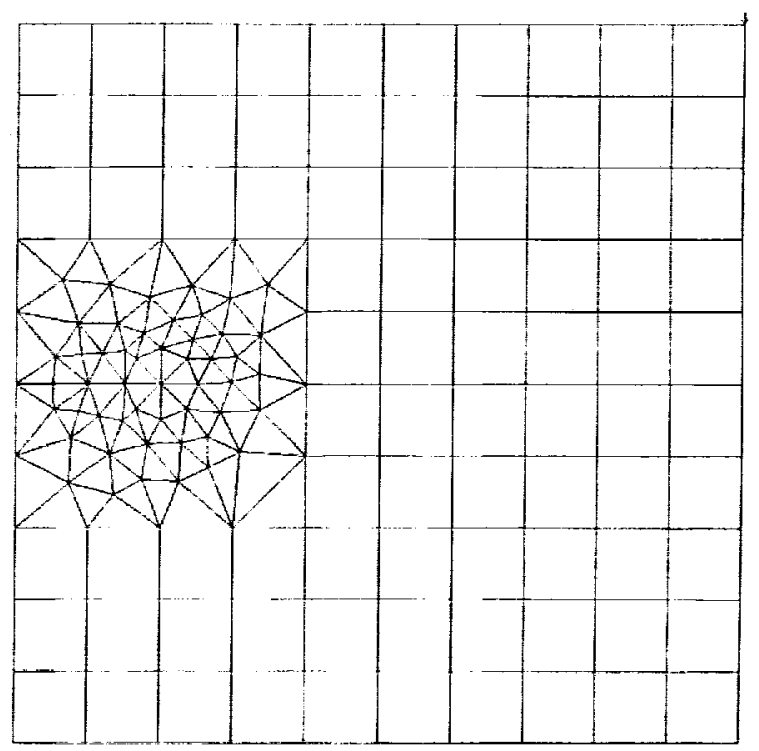

b) Finite element mesh after adding initial crack

Figure 3.8: Initial edge crack in model.

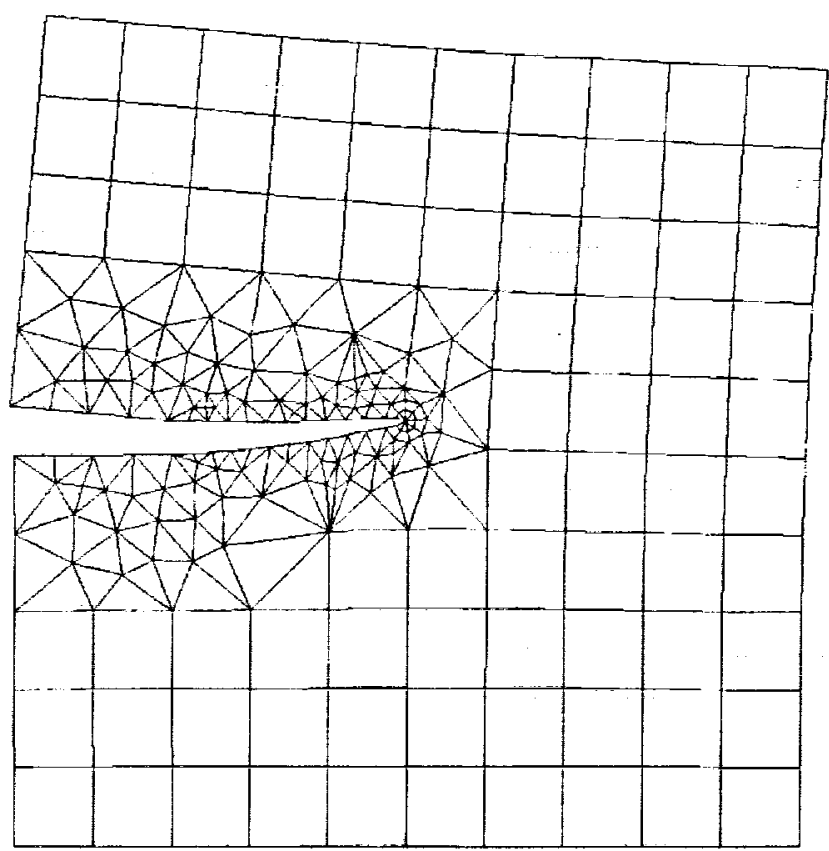

Figure 3.9: Predicted crack trajectory for model in Figure 3.8.

Figure 3.9 is a picture of the predicted crack trajectory for the finite element model in Figure 3.8. The crack has been incrementally advanced from the initial 
length and orientation through five propagation steps. For the assumed material properties and loading in this example, the calculated number of load cycles to grow the crack from the initial length in Figure 3.8 to that in Figure 3.9 is 4,900 cycles.

\subsubsection{Example: Three dimensional, mode I dominant fatigue crack growth simulation with static, proportional loading}

The assumptions of the two dimensional example will apply to this three dimensional example. In three dimensions, the procedure to predict fatigue crack trajectories is very similar to that in two dimensions. As in two dimensions, the geometry model must be defined, the mesh created, and the model attributes assigned. The main complexity with three dimensional crack growth simulations is that there is not a single crack tip, but rather a three dimensional crack front. For a given three dimensional crack, there is no longer a single value for the SIF in each mode, but rather a SIF distribution along the crack front for each mode. In addition, the crack length might also vary along the crack front.

In this thesis, all of the three dimensional models are boundary element models. In the boundary element method, the primary variables are load and displacement. Strains and stresses are secondary variables. The BEM is based on an integral equation formulation. An advantage of the method is that the number of unknowns in the equations is proportional to the surface discretization. This is in contrast to the FEM where the number of unknowns is proportional to the volume discretization. In computational fracture mechanics when predicting crack trajectories and remeshing are necessary, an advantage of the BEM is that only the surfaces near the crack need to be remeshed, as opposed to the entire volume which must be remeshed when using the FEM. Volume meshing with cracks can be rather difficult; whereas, surface meshes are straightforward with and without cracks.

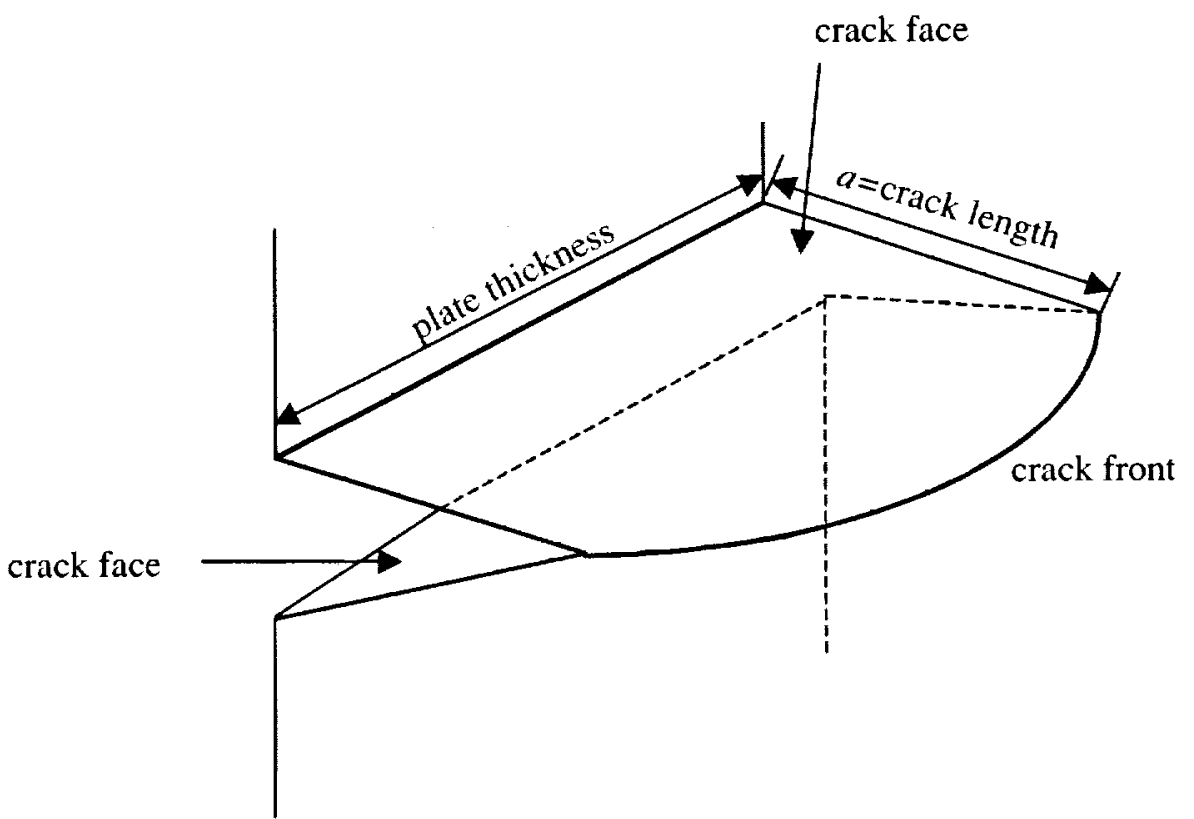

Figure 3.10: Schematic of three dimensional crack front. 
There are no closed form solutions to calculate SIF distributions along the crack front for arbitrary three dimensional cracks. As a result, a conventional approach to calculate the SIF distribution is to discretize the front into a series of two dimensional crack tips. For example, the finite plate model presented in Section 3.2.2, in reality, has a finite width. Therefore, the crack must have a finite width. The crack front shape might be that shown in Figure 3.10. In this example, the crack width is equal to the plate thickness.

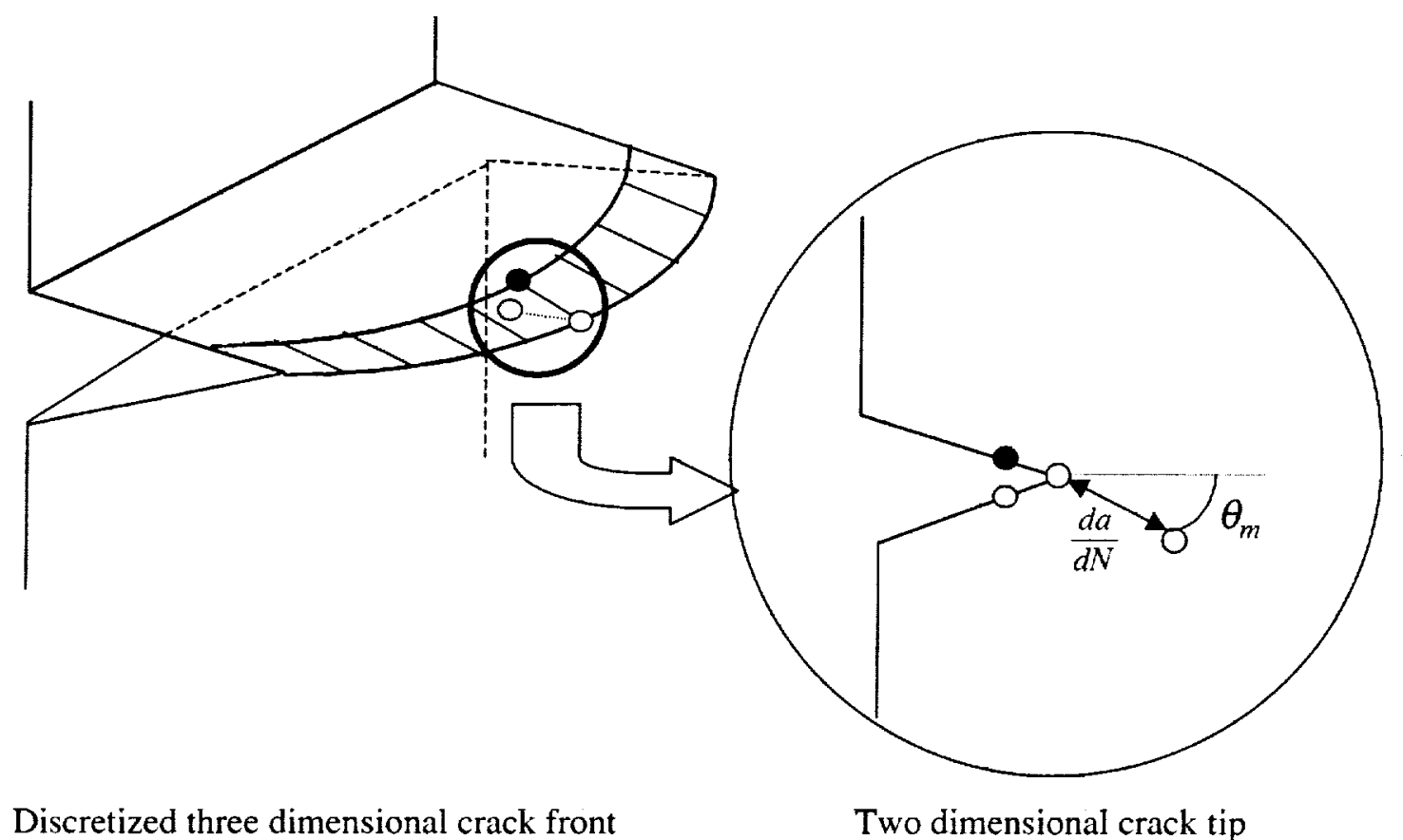

Figure 3.11: Discrete crack front points treated as two dimensional problems.

Next, the crack front is discretized, as shown by the lines intersecting the crack front in Figure 3.11. Once the crack front is discretized, each point is treated as a two dimensional problem. The two dimensional methods to calculate SIFs are applied at each discrete point. The discrete point is propagated by an amount and an angle uniquely defined by the SIFs associated with that point. Once each discrete crack front point is propagated individually, a least squares curve fit is performed through the new discrete crack front points, Figure 3.12.

A potential difference in the three dimensional approach, as opposed to the two dimensional method, is that singular crack front elements might not be used along the crack front. Since the BEM is implemented in this thesis, the volume of the three dimensional model is not meshed; only the surfaces are meshed. Therefore, elements that represent the crack tip singularity are not available along the crack front. The main drawback of this is that some SIF accuracy along the crack front is sacrificed. 


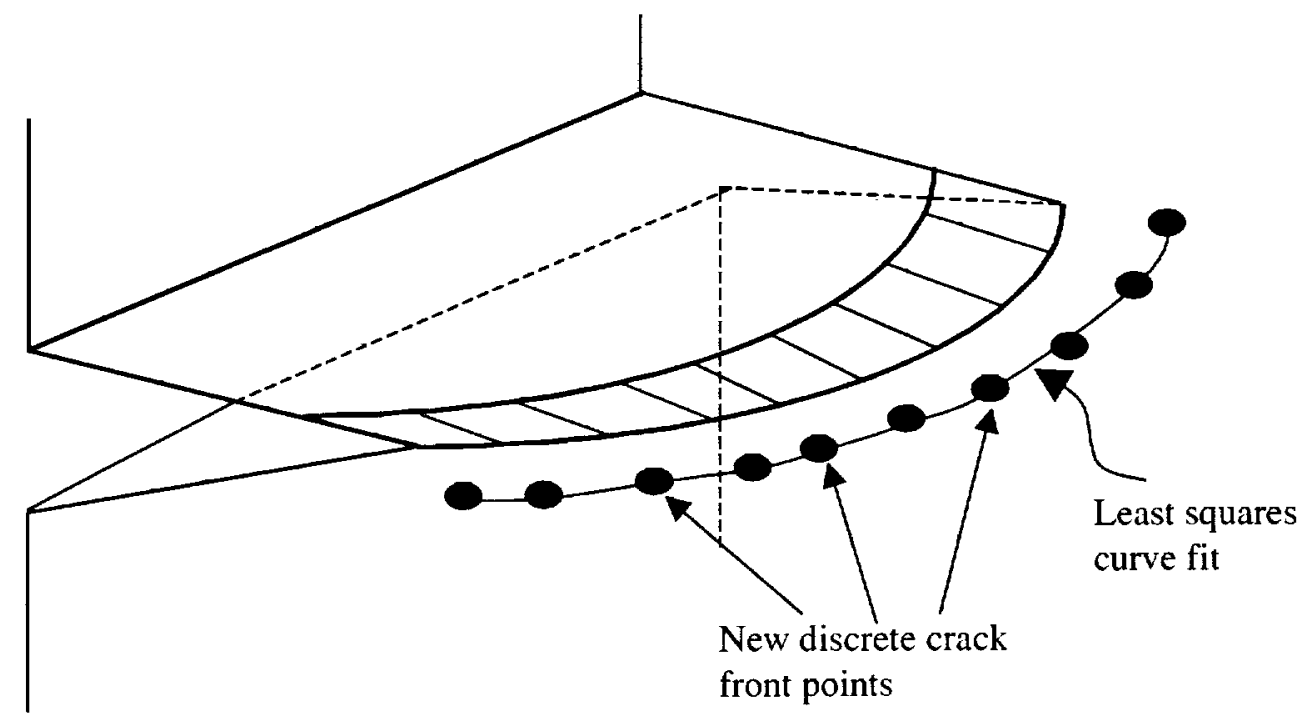

Figure 3.12: Least squares curve fit through new discrete crack front points.

\subsection{Fracture Mechanics Software}

A suite of fracture mechanics software developed by the Cornell Fracture Group is used in this thesis [FRANC3D 1999a, 1999b]. The codes were developed to handle the complexities of three dimensional crack trajectory predictions. OSM is used to define a three dimensional solid geometry model of an object. The program is based on defining the surfaces of the model explicitly in Cartesian space. The boundary of a solid is generated by adjacent surfaces, or faces. Each face of the boundary element model has a three dimensional local coordinate system associated with it. In order to define a closed solid, all of the local face normals must point away from the interior of the solid. The local coordinate system might also be of significance when defining boundary conditions.

The geometry model is then read into FRANC3D. With FRANC3D, a user can create a finite element or boundary element mesh based on the geometry model. Displacement or force/traction boundary conditions must be defined for all the faces of the solid. The conditions must be specified in all three Cartesian directions with respect to either the local or the global coordinate system. Material properties are also assigned to regions of the model using FRANC3D.

Cracks are added to the solid by explicitly defining the vertices, edges, and faces that model the cracks. A crack has two distinct faces that must be meshed identically.

As mentioned in Section 3.2.3, a crack front must be discretized prior to calculating SIFs and to propagating the crack. Within FRANC3D, there are three options to discretize the crack front. The discrete points can be defined by the mesh nodes, the midpoints of the elements sides along the crack, or at a user defined number of equally spaced points along the crack front. The built in feature in FRANC3D to calculate SIFs uses the displacement correlation technique. The most accurate results are obtained when a row of four sided elements is used along the crack front. This will give a set of equally spaced points behind the crack front where the SIFs can be 
evaluated. Additionally, to improve the performance of the crack front elements, the ratio of the elements' width to length should be close to one [FRANC3D 1999c].

When a crack is propagated, the geometry model changes. However, the geometry changes only near the crack. Therefore, only the mesh model near the new crack is damaged and requires remeshing. The remainder of the geometry and mesh model is left unchanged. This is a distinct advantage of FRANC3D.

The program BES is used to solve for the displacements and stresses using the boundary element technique. FRANC3D is used as a post-processor to view the deformed shape, stress contours, and extract nodal information.

FRANC3D uses the same functional form to interpolate the geometry and field variable variations over an element. The form is given by the associated element type. In all of the models, only isoparametric three- and four-noded elements are used. Quadratic elements are available; however, based on the work in [FRANC3D 1999c], the gain in accuracy does not justify the significant increase in computational time.

\subsection{Chapter Summary}

This chapter covered theories of LEFM and fatigue pertinent to modeling crack growth numerically. Of primary importance is how crack growth rates and trajectory angles are calculated from SIFs. The maximum principal stress theory will be used to calculate trajectories under mixed mode loading. In addition, the displacement correlation method was introduced as a technique to evaluate SIFs. Two dimensional and three dimensional examples demonstrated how the theories are applied in numerical simulations. Some features of the software programs FRANC3D, OSM, and BES that will be used in the simulations were covered. The background provided in Chapter 2 and this chapter will be utilized in the work of Chapters 4,5 , and 6 . The studies in those chapters cover issues related to predicting three dimensional fatigue crack trajectories in a spiral bevel gear. 


\section{CHAPTER FOUR: FATIGUE CRACK GROWTH RATES}

\subsection{Introduction}

The goal of this chapter is to determine how highly negative stress ratios affect the fatigue crack growth rate in a common gear steel, AISI 9310. This is of interest in the context of gears because the magnitude of compressive stresses in a gear's tooth root is a function of the rim thickness. If fatigue crack growth rates are highly sensitive to compression, then crack growth rates may warrant more attention in designing gears. On the other hand, if the compressive stresses do not alter the fatigue crack growth rate predictions greatly, than the loading cycle for a gear tooth can be simplified by ignoring the compressive portion of the cycle.

In Section 4.2, the concept of fatigue crack closure is discussed. This section shows that crack closure provides a convenient framework within which to understand the factors that control fatigue crack growth. A material-independent method is presented for obtaining fatigue crack growth rate data that does not vary with stress ratio, $R$. The crack closure approach is extended beyond aluminum alloys, considered by Elber [1971] and Newman [1981] and discussed in Section 4.2, to steels. Next, Section 4.3 applies the concepts to AISI 9310 data to obtain an intrinsic fatigue crack growth model. Section 4.4 demonstrates that in the range of negative $R$, the effective stress range, and likewise the crack growth rate, is not highly sensitive to the magnitude of $R$.

\subsection{Fatigue Crack Closure Concept}

Due to the cyclical loading on a gear's tooth, fatigue crack propagation might occur. The load range, $\Delta S$, or stress intensity factor range, $\Delta K$, along with the load ratio, $R$, characterizes cyclic loading. Recall, $R$ is defined as the ratio of minimum stress, $S_{\text {min }}$, to maximum stress, $S_{\max }$, which, due to similitude, is equal to the ratio of minimum mode I SIF, $K_{\text {min }}$, to maximum mode I SIF, $K_{\max }$ (Equation 3.5).

Lewicki $e$ al. [1997b] found that spur gear teeth can have $R$-values as low as -3.0. They also found that the magnitude of $R$ in spur gears is a function of the gear geometry. As the rim thickness decreases, $R$ becomes more negative due to the increased bending of the gear rim.

A general interpretation of the crack closure approach is that damage only occurs during the portion of the load cycle when the crack faces are not in contact. The majority of the literature's discussion of crack closure covers its effect on crack growth rates. Since gears have such high load frequencies, crack growth rates are commonly of secondary interest in the context of gears. The time from detectable flaw to failure is usually insignificant. However, if the crack growth rate is highly sensitive to the magnitude of the compressive portion of the load cycle, then crack growth rates may warrant more attention. On the other hand, if, for negative values of $R$, the crack growth rate is relatively insensitive to the magnitude of $R$, then the effect of geometry on $R$ need not be the primary concern in gear design. This demonstration 
is significant in the context of the overall goal of this thesis, which is to study aspects of gear geometry that affect damage tolerance.

It is assumed initially in this chapter that the stresses induced in a gear tooth under positive (tensile) and negative (compressive) parts of the load cycle are "proportional." In other words, the shape of the stress intensity factor distribution along the crack front is the same under both tensile and compressive loading. In two dimensional analyses, this is not a concern because the crack only consists of a tip, where the deformation can be tensile only or compressive only, not a combination of the two. In three dimensions, however, the distribution of the loading (deformation) along the crack front might be different in the compressive and tensile load cases. In the end, whether the positive and negative parts of the load cycle are proportional is not of major concern. As will be shown in the remaining sections, damage occurs only during the tensile portion of the load cycle.

Elber [1971] observed that during unloading a crack actually closes prior to the applied load being entirely removed. This phenomenon has been called fatigue crack closure. Fatigue crack closure also explains why, for a given $\Delta K$, fatigue tests show the crack growth rate increasing as $R$ increases. Figure 4.1 shows typical fatigue crack growth rate data as a function of SIF range [Kurihara et al. 1986]. Kurihara et al. conducted fatigue tests with $500 \mathrm{MPa}$ class C-Mn steel, which is used in pressure vessels. The tests covered a wide range of stress ratios from -5.0 to 0.8 . Figure 4.1 was obtained by selecting two data points off Kurihara et al.'s plots for each value of $R$. The horizontal scatter in the curves is a result of the different $R$-values. Note that as $R$ increases, the curves shift to the left, producing an increase in fatigue crack growth rate for a given $\Delta K$.

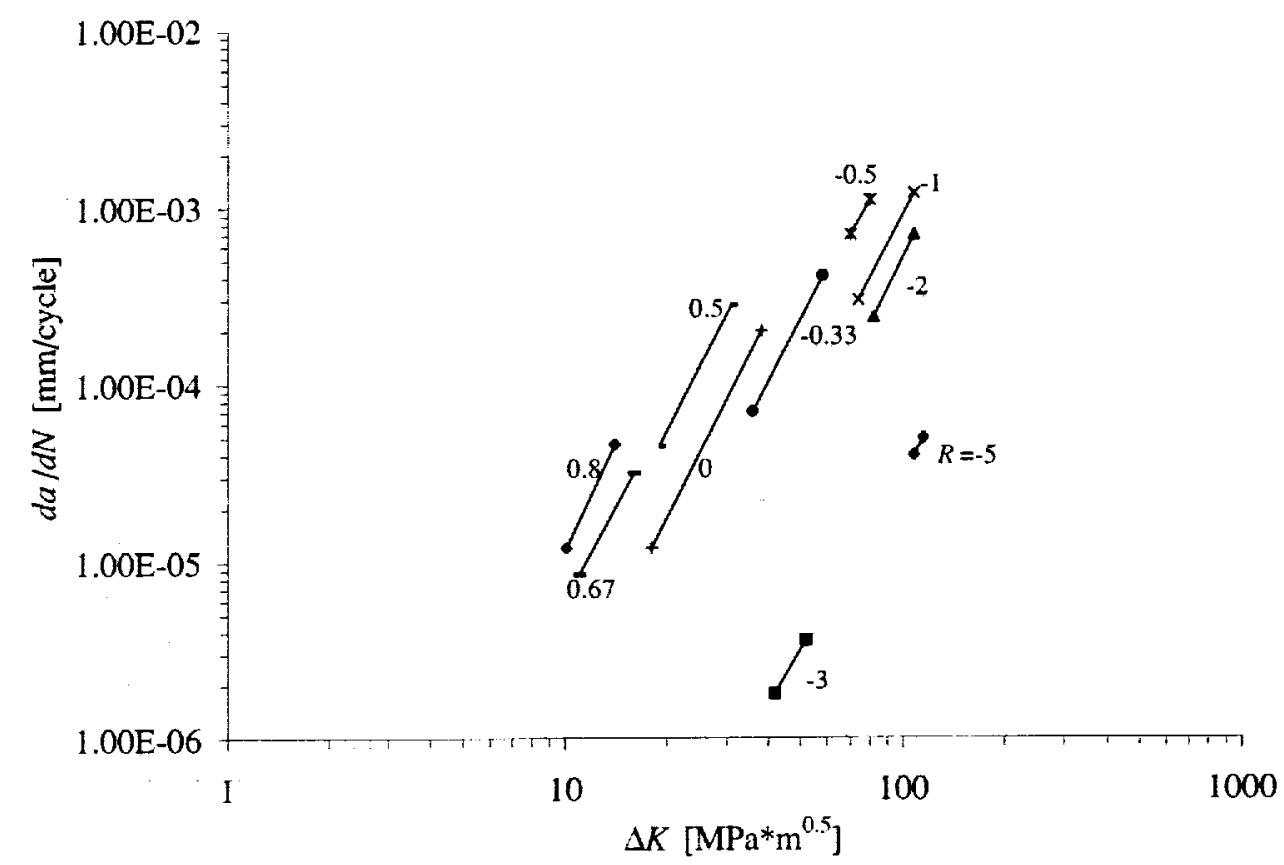

Figure 4.1: Fatigue crack growth rate data for pressure vessel steel at various $R$-values (data taken from [Kurihara et al. 1986]). 
Crack closure can be attributed to a number of factors. During the opening portion of a load cycle, the material at the crack tip plastically deforms. As the cycles repeat, a wake of plastic deformation remains as the crack propagates through the body. The plastic deformation wake results in a mismatch between the crack faces. Although not considered here, crack closure can also occur due to differences in the surface roughness of the crack faces, due to mixed mode loading, or oxidation of the crack surfaces.

Elber modified Paris' model to account for crack closure. The modification allows crack propagation to occur only while the crack tip is open. He introduced $S_{o p}$ as the stress level where the crack first opens during the tensile part of the load cycle. His equation for the crack propagation rate is:

$$
\frac{d a}{d N}=C\left(\Delta K_{\text {off }}\right)^{n}=C(U \Delta K)^{n}
$$

where $U$, the effective stress range ratio, is defined as

$$
U=\frac{S_{\text {max }}-S_{o p}}{S_{\text {max }}-S_{\text {min }}}=\frac{1-S_{\text {iv }} / S_{\text {max }}}{1-R}
$$

Figure 4.2 illustrates the relationships among various $K$ values. $S_{o p}\left(K_{o p}\right)$ is difficult to measure experimentally. In addition, the value varies with loading conditions. As a result, Elber developed an empirical relationship between $U$ and $R$. From this relationship, $S_{o p}\left(K_{o p}\right)$ could be backed out.

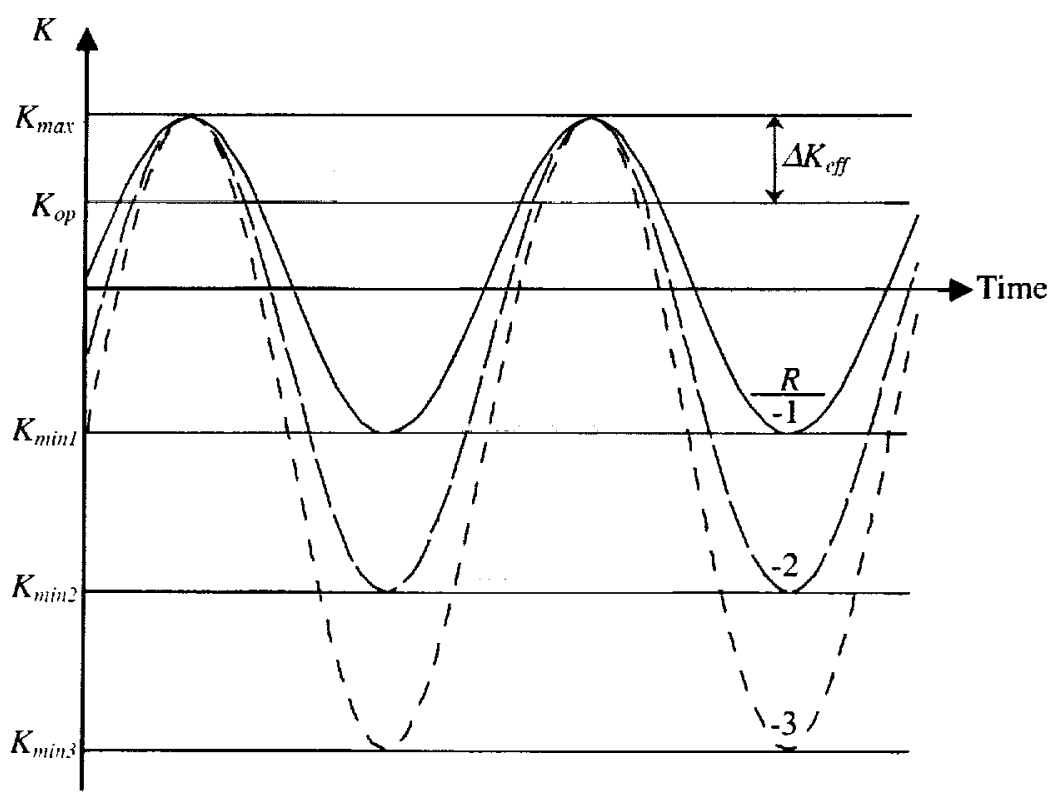

Figure 4.2: Constant $\Delta K_{\text {eff }}$ for different stress ratios.

When $d a / d N$ is plotted as a function of $\Delta K_{\text {eff }}$, the scattered curves (due to different $R$-values) collapse into a single, "intrinsic" crack growth rate curve. In crack-closure-based fatigue models, $d a / d N$ is a function of $\Delta K_{\text {cff. }}$. This implies that crack growth occurs only while the crack tip is open. If $K_{\max }$ is kept constant between various tests with different $R$-values, then $K_{\text {min }}$ must change. If it can be shown that 
$\Delta K_{\text {eff }}$ remains nearly constant for various negative $R$-values, then the portion of the load cycle when $K_{\text {min }} \leq K_{o p}$ does not contribute to crack growth. Therefore, all negative $R$-value cases could be treated in the same manner. The sensitivity of $\Delta K_{e f f}$ will be investigated in Section 4.4. Figure 4.2 illustrates how $\Delta K_{\text {iff }}$ could remain constant as $K_{\text {min }}$ decreases.

Elber performed a series of experimental fatigue tests with sheets of 2024-T3 aluminum alloy. The stress ratio range was $-0.1 \leq R \leq 0.7$. From the tests, he developed the empirical relationship

$$
U(R)=0.5+0.4 R \quad \text { when }-0.1 \leq R \leq 0.7
$$

Elber's $U(R)$ relationship is valid only for 2024-T3 aluminum alloy over the range of $R$-values for which he had experimental data. His work inspired many to develop empirical relationships between $U$ and $R$ for a variety of materials and ranges of $R$. Schijve [1988] summarizes several of these empirical relationships for different alloys and ranges of $R$. However, it is expensive to develop this relationship empirically every time one wants to model crack closure in a new material. This led to attempts to numerically model crack closure [Newman 1976, 1981], [Fleck et al. 1988], [McClung et al. 1989], and [Blom et al. 1985]. Through the thirty plus years of research related to crack closure, it has been found that the amount of crack closure is dependent on many variables. Specimen size, specimen geometry, crack length, applied stress state, and prior loading conditions all affect the magnitude of $S_{o p}$. Newman's work attempts to incorporate all of these factors.

Newman developed and applied a hybrid analytical/numerical crack closure model that simulated plane strain and plane stress conditions. He successfully matched crack growth rates under constant-amplitude loading from his analytical model to experimental data. The material he focused on initially was 2219-T851 aluminum alloy. The model has since been applied to a variety of metals. Newman's model is the most comprehensive and has been successfully validated against experiments. As a result, his model will be utilized in this thesis.

All variables in Equation (4.2) are defined immediately from the loading conditions with the exception of $S_{o p}$. To find an expression for $S_{o p} / S_{\text {ma. }}$, Newman [1984] fit equations to his numerical results for 2043-T3 aluminum alloy over a large range of $R$ values and load levels. He worked in terms of applied loads, but due to similitude, $S$ in his expressions can be replaced with $K$, giving:

$$
\begin{array}{ll}
\frac{K_{o p}}{K_{\text {max }}}=A_{0}+A_{1} R+A_{2} R^{2}+A_{3} R^{3} & \text { for } R \geq 0 \\
\frac{K_{o p}}{K_{\text {max }}}=A_{0}+A_{1} R & \text { for }-1 \leq R<0
\end{array}
$$

when $K_{o p} \geq K_{\min }$. The coefficients $A_{0}-A_{3}$ are:

$$
A_{0}=\left(0.825-0.34 \kappa+0.05 \kappa^{2}\right)\left[\cos \left(\frac{\pi S_{\max }}{2 \sigma_{0}}\right)\right]^{1 / \kappa}
$$




$$
\begin{aligned}
A_{1} & =\left(0.415 \kappa-0.071 \kappa^{2}\right) \frac{S_{\max }}{\sigma_{0}} \\
A_{2} & =1-A_{0}-A_{1}-A_{3} \\
A_{3} & =2 A_{0}+A_{1}-1
\end{aligned}
$$

$\kappa$ is a constraint factor taking on a lower bound of 1 for plane stress conditions and an upper bound value of 3 to simulate plane strain conditions. The flow stress, $\sigma_{0}$, is the average between the uniaxial yield stress and the uniaxial ultimate tensile strength of the material.

Because Newman's model for $K_{o p}$ is a function of material constants $\left(\sigma_{0}\right), R$, and $\kappa$, it is applicable for any fatigue crack where LEFM holds and the loading conditions and material properties are known. Figure 4.3 is an example of how the curves in Figure 4.1 collapse into an intrinsic curve when crack closure is taken into account. Equations (4.4) and (4.5) are used to calculate $\Delta K_{o p} . U$ is calculated using Equation (4.2). ${ }^{5}$

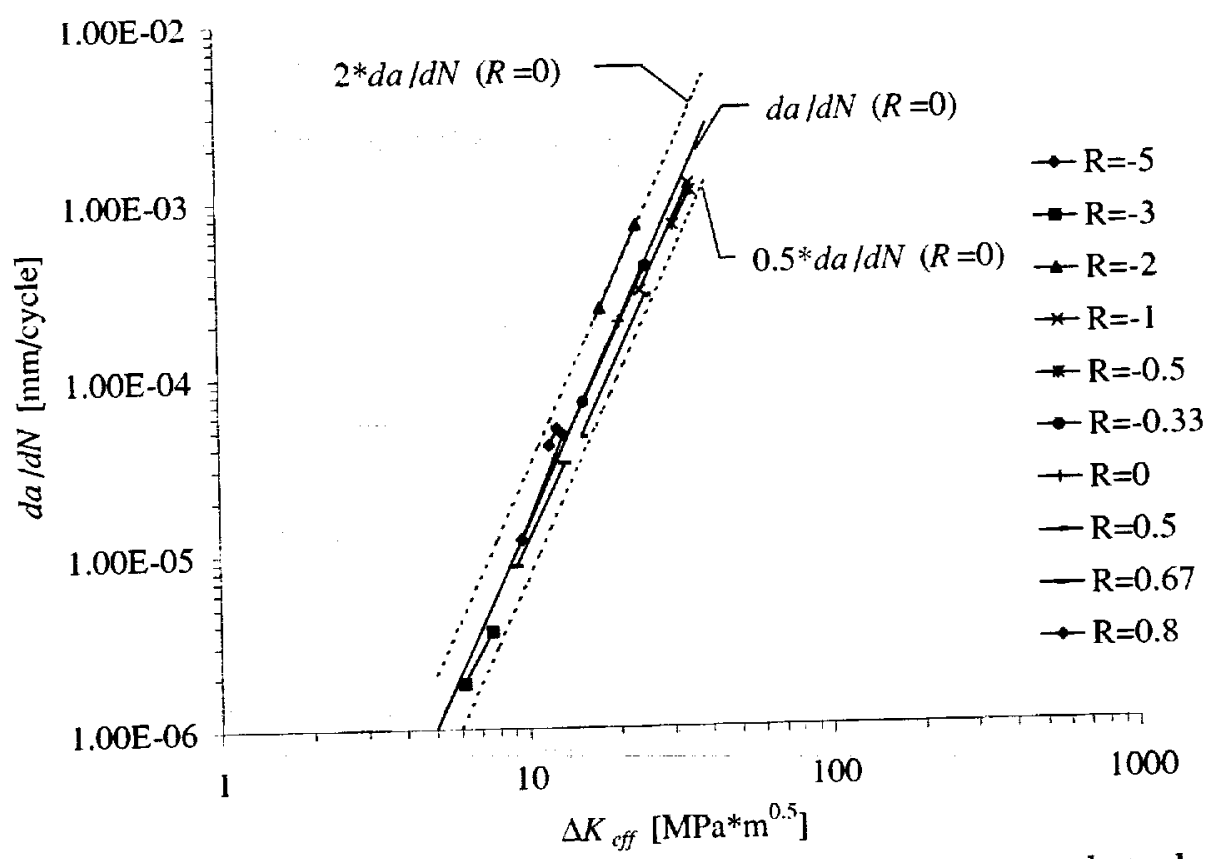

Figure 4.3: Intrinsic fatigue crack growth rate data for pressure vessel steel using Newman's equations for $\Delta K_{\text {eff }} ; \kappa=1$ (using data taken from [Kurihara et al. 1986]).

The crack tip condition in the fatigue test specimen Kurihara et al. used, a thin plate with a center crack, is best described by plane stress. Therefore, a value of $\kappa=1$ was selected for the preliminary graphs. $\kappa$ was then increased, and the amount

${ }^{5}$ Note that Newman claims Equation (4.4b) is valid for negative $R$-values greater than or equal to -1 . However, Kurihara et al.'s data extends to -5.0 . Equation $(4.4 \mathrm{~b})$ was used for the cases when $R=-5.0$, -3.0 , and -2.0 . Figure 4.3 illustrates, at least for this case, the equation can also hold for these low $R$ values. 
of correlation between the curves was visually inspected. As $\kappa$ increased, the curves became more scattered, validating the choice of $K=1$.

The equation of a line in Figure 4.3 is given by:

$$
\log \left(\frac{d a}{d N}\right)=n^{*} \log \left(\Delta K_{\text {eff }}\right)+\log \left(C^{*}\right)
$$

In Figures 4.1 and 4.3 , the slope for a given curve ( $R$-value) is uniquely defined by the data points. According to the crack growth models, all of the curves should have the same slope. Ideally, this would be the case for the plots in Figure 4.3. The relatively small scatter in the magnitude of the slopes at different $R$-values is attributed to scatter in the experimental results.

Figure 4.3 includes the intrinsic curve predicted by the intrinsic $R=0$ data. This curve falls roughly in the middle of the predicted curves. To give an idea of the scatter in the curves, the figure also includes lines corresponding to one half and two times the crack growth rate for $R=0$. All of the predicted intrinsic curves fall into this envelope. As a result, it is concluded that the $\Delta K_{\text {eff }}$ equations produce good correlation.

These results with $500 \mathrm{MPa}$ pressure vessel steel demonstrate that an intrinsic fatigue crack growth rate curve can be obtained using Newman's material-independent model to account for crack closure. It is also shown that a possibility exists to extend the model beyond the range of $R \geq-1$. Consequently, in Section 4.3 the model will be applied to AISI 9310 steel to determine how negative $R$-values influence crack propagation rates.

\subsection{Application of Newman's Model to AISI 9310 Steel}

An open literature search for fatigue crack growth rate data for AISI 9310 steel at various $R$-values revealed little published information. A report by Au et al. [1981] contains the most information. Au et al. performed tests in different environments at various $R$-values and frequencies for carburized and noncarburized AISI 9310 steel. Because they were investigating the correlation between fatigue striations and crack growth rates, only two tests were performed on noncarburized steel in the same environment and at the same load frequency but at different $R$-values. The load levels used in the tests were not reported. When their measured fatigue crack growth rates at $R=0.05$ and 0.5 are plotted against $\Delta K$, there is very little scatter in the curves. This suggests that the crack growth rate is not sensitive to $R$ or that the applied loads were high enough such that $K_{o p} \leq K_{m i n}$. Since the objective of this study is to correlate fatigue crack growth rate data at different $R$-values, including the negative $R$ regime, Au et al.'s data is inadequate.

Additional fatigue test data for AISI 9310 was provided by a helicopter manufacturer on the condition that the data's source not be identified. Data points are extracted from the fatigue crack growth rate curves obtained from tests in two different environments. Figure 4.4 shows growth rates for AISI 9310 steel in room temperature air for $R=-1,0.05$, and 0.5 . The curves in Figure 4.5 are obtained by extracting data points from fatigue crack growth rate tests in $250^{\circ}$ oil for $R=-1,0.01$, and 0.5. Table 4.1 summarizes the slopes and intercepts for the various curves. 


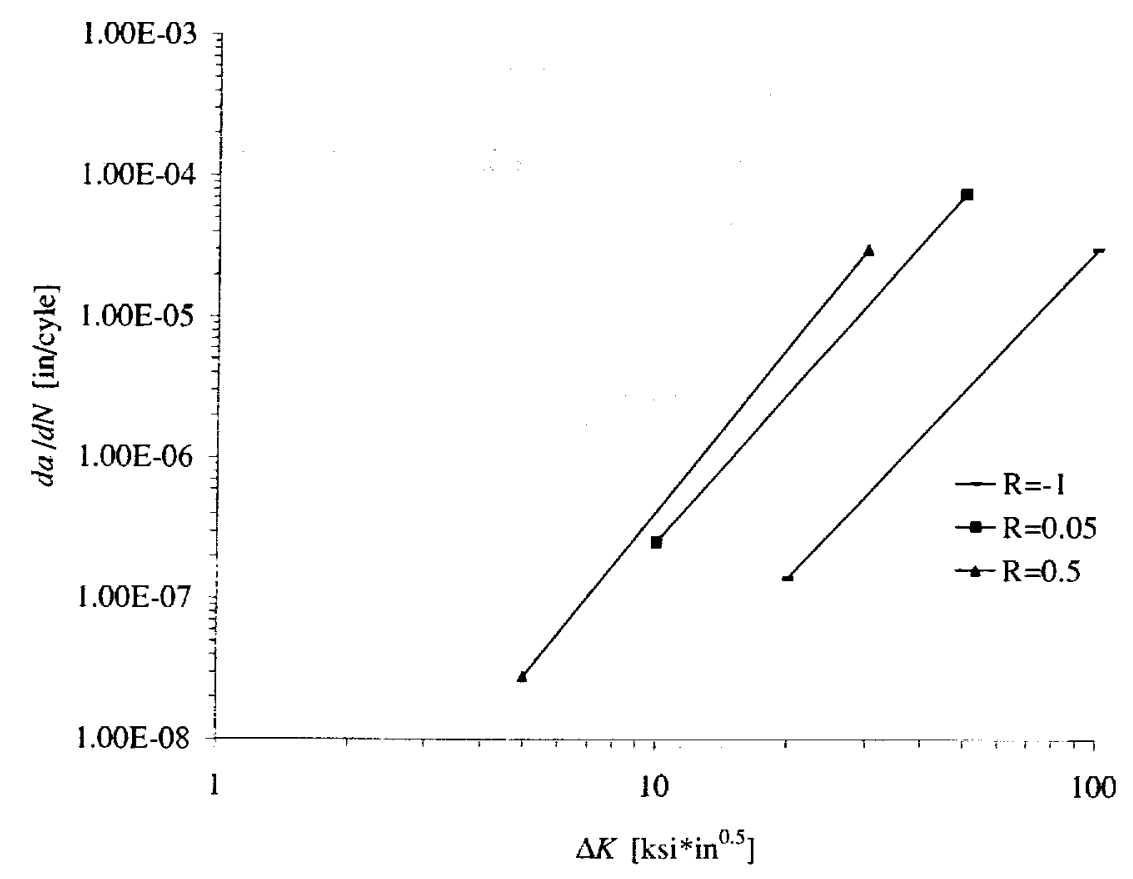

Figure 4.4: Fatigue crack growth rate data for AISI 9310 steel in room temperature air.

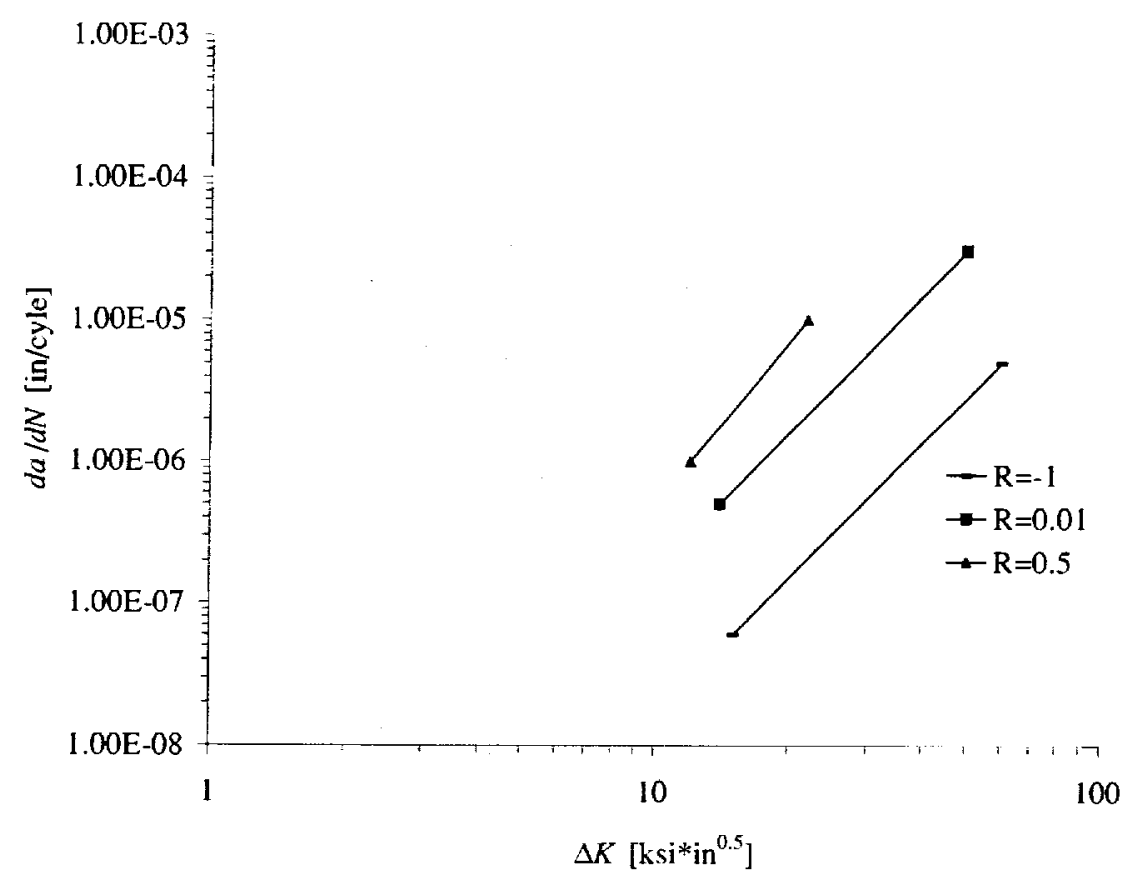

Figure 4.5: Fatigue crack growth rate data for AISI 9310 steel in $250^{\circ}$ oil. 
Table 4.1: Slope and intercept of curves in Figures 4.4 and 4.5.

\begin{tabular}{|c|c|c|}
\hline Test & $\mathbf{n}$ & $\begin{array}{l}\mathbf{C} \\
{\left[(\text { in/cycle }) /\left(\mathrm{ksi}^{*} i \mathrm{in}^{0.5}\right)^{\mathrm{n}}\right]}\end{array}$ \\
\hline$R=-1 \quad$ (Air) & 3.3 & $6.4 e-12$ \\
\hline$R=0.05$ (Air) & 3.5 & $7.3 e-11$ \\
\hline$R=0.5 \quad$ (Air) & 3.9 & $5.3 e-11$ \\
\hline$R=-1 \quad$ (Oil) & $\overline{3.2}$ & $1.1 \mathrm{e}-11$ \\
\hline$R=0.01($ Oil) & 3.2 & $9.9 \mathrm{e}-11$ \\
\hline$R=0.5 \quad(\mathrm{Oil})$ & 3.8 & $7.9 \mathrm{e}-11$ \\
\hline
\end{tabular}

For a given $R$, the $n$ values are similar between the two environments. The effect of the environment can be see in the variations of $C$. $C$ is consistently larger in the heated oil environment. A larger $C$ will result in faster growth rates. However, the environment effect will not be considered in this investigation.

Similar to the pressure vessel steel analyses, da/dN versus $\Delta K_{\text {eff }}$ plots are generated using Equations (4.2), (4.4), and (4.5). A value of $\kappa=1$ best describes the condition at the crack tip in the test specimen. Figures 4.6 and 4.7 illustrate the various curves collapsing into an intrinsic fatigue crack growth rate curve.

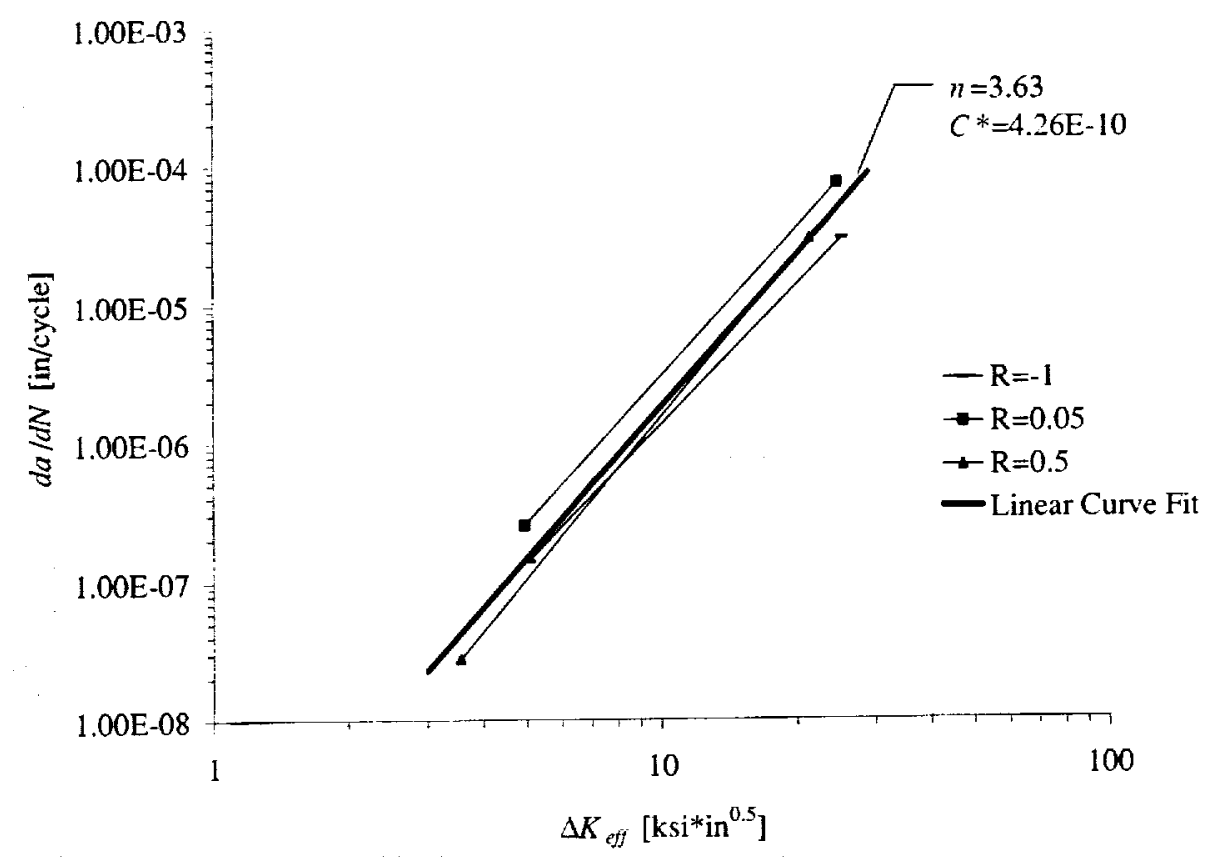

Figure 4.6: Intrinsic fatigue crack growth rate for AISI 9310 in room temperature air,

$$
\kappa=1 \text {. }
$$

Figures 4.4 through 4.7 demonstrate that Newman's crack closure model accounts for the scatter in fatigue crack growth rates at different stress ratios in AISI 9310 steel. Table 4.2 contains the slopes and vertical intercepts from the lines in the figures. In addition, a linear least squares curve is fit through the data in Figures 4.6 and 4.7. The slope and vertical intercept from each curve fit are also included in the 
table. These curve fit values will be used in the crack growth rate models for the numerical analyses. Crack closure concepts will now be extended to investigate the sensitivity of fatigue crack growth rates to low $R$-values.

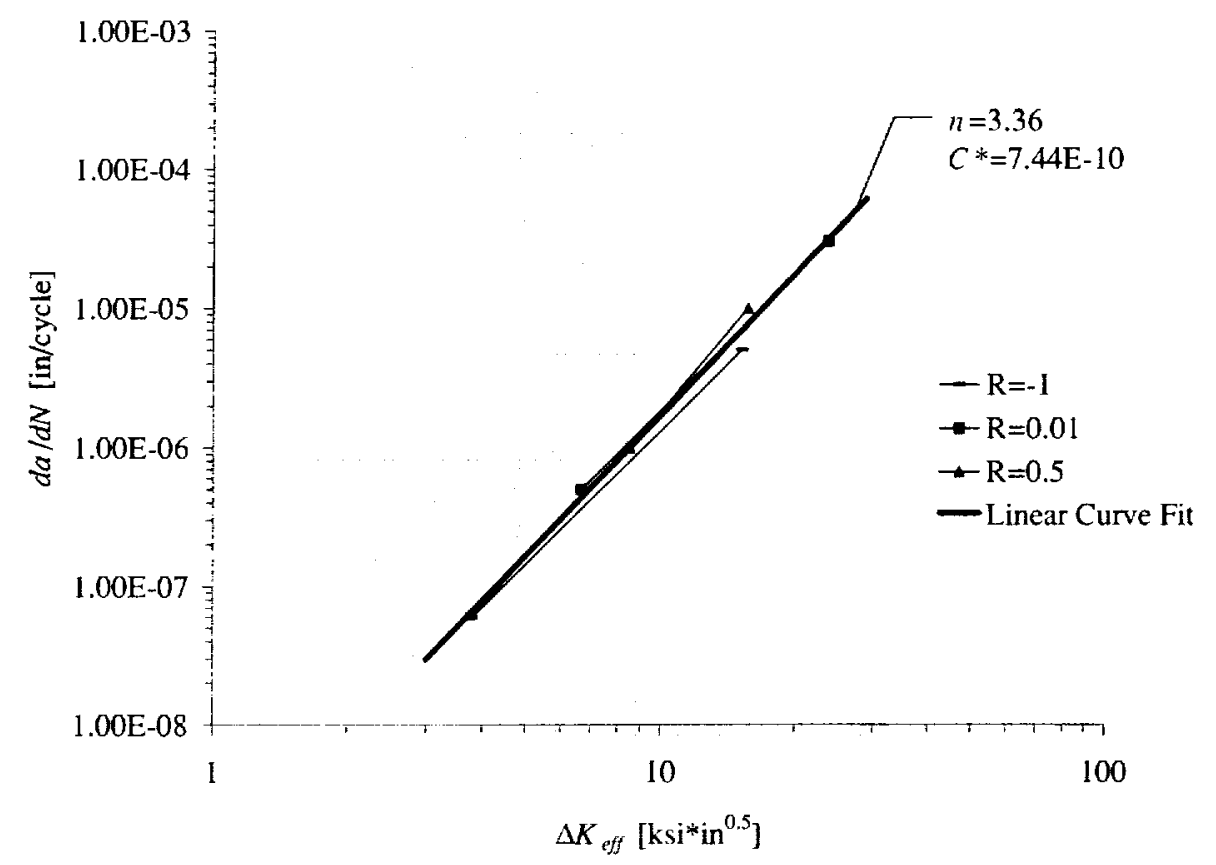

Figure 4.7: Intrinsic fatigue crack growth rate for AISI 9310 in $250^{\circ}$ oil; $\kappa=1$.

Table 4.2: Intrinsic and non-intrinsic fatigue crack growth rate parameters.

\begin{tabular}{|c|c|c|c|}
\hline Test & $\mathbf{n}$ & $\begin{array}{l}\mathrm{C} \\
{\left[(\mathrm{in} / \mathrm{cycle}) /\left(k \mathrm{si}^{*} \mathrm{in}^{0.5}\right)^{\mathrm{n}}\right]}\end{array}$ & $\begin{array}{l}\mathrm{C}^{*} \\
{\left[(\text { in/cycle }) /\left(k s i^{*} i n^{0.5}\right)^{\mathrm{n}}\right]}\end{array}$ \\
\hline$R=-1 \quad$ (Air) & 3.3 & $6.4 \mathrm{e}-12$ & $6.30 \mathrm{e}-10$ \\
\hline$R=0.05$ (Air) & 3.5 & $7.3 e-11$ & $8.80 \mathrm{e}-10$ \\
\hline$R=0.5 \quad($ Air $)$ & 3.9 & $5.3 e-11$ & $1.98 \mathrm{e}-10$ \\
\hline$R=-1 \quad($ Oil $)$ & 3.2 & $1.1 \mathrm{e}-11$ & $8.52 \mathrm{e}-10$ \\
\hline$R=0.01(\mathrm{Oil})$ & 3.2 & $9.9 e-11$ & $1.09 \mathrm{e}-9$ \\
\hline$R=0.5 \quad(\mathrm{Oil})$ & 3.8 & $7.9 \mathrm{e}-11$ & $2.87 \mathrm{e}-10$ \\
\hline Curve Fit Air & 3.6 & $\mathrm{NA}^{6}$ & $4.26 \mathrm{e}-10$ \\
\hline Curve Fit Oil & 3.4 & $\mathrm{NA}^{1}$ & $7.44 \mathrm{e}-10$ \\
\hline
\end{tabular}

${ }^{6}$ Not Applicable 


\subsection{Sensitivity of Growth Rate to Low $R$}

Table 4.3 contains results from calculations of $\Delta K_{\text {eff }}$ at different $R$-values using Equations (4.1), (4.2), (4.4), and (4.5). Constant values for $K_{\max }, \kappa$, and $S_{\max } / \sigma_{0}$ are assumed.

Table 4.3: Calculations to find $\Delta K_{\text {eff }}$ over a range of $R$-values for a constant $K_{\text {max }}$; SIF units are $\mathrm{ksi}^{*} \mathrm{in}^{0.5}$.

\begin{tabular}{|r|c|c|c|c|c|c|c|}
\hline $\mathrm{R}$ & $\kappa$ & $S_{\max } / \sigma_{0}$ & $\boldsymbol{K}_{\max }$ & $\boldsymbol{K}_{\min }$ & $\Delta \boldsymbol{K}$ & $\mathrm{U}$ & $\Delta \boldsymbol{K}_{\text {eff }}$ \\
\hline 0.705 & 1.000 & 0.100 & 10.000 & 7.050 & 2.950 & 0.825 & 2.434 \\
\hline 0.700 & 1.000 & 0.100 & 10.000 & 7.000 & 3.000 & 0.822 & 2.467 \\
\hline 0.505 & 1.000 & 0.100 & 10.000 & 5.050 & 4.950 & 0.716 & 3.542 \\
\hline 0.500 & 1.000 & 0.100 & 10.000 & 5.000 & 5.000 & 0.713 & 3.565 \\
\hline 0.255 & 1.000 & 0.100 & 10.000 & 2.550 & 7.450 & 0.589 & 4.388 \\
\hline 0.250 & 1.000 & 0.100 & 10.000 & 2.500 & 7.500 & 0.587 & 4.399 \\
\hline 0.005 & 1.000 & 0.100 & 10.000 & 0.050 & 9.950 & 0.474 & 4.714 \\
\hline 0.000 & 1.000 & 0.100 & 10.000 & 0.000 & 10.000 & 0.472 & 4.716 \\
\hline-0.495 & 1.000 & 0.100 & 10.000 & -4.950 & 14.950 & 0.327 & 4.886 \\
\hline-0.500 & 1.000 & 0.100 & 10.000 & -5.00 & 15.000 & 0.326 & 4.888 \\
\hline-0.955 & 1.000 & 0.100 & 10.000 & -9.950 & 19.950 & 0.254 & 5.058 \\
\hline-1.000 & 1.000 & 0.100 & 10.000 & -10.000 & 20.000 & 0.253 & 5.060 \\
\hline-1.995 & 1.000 & 0.100 & 10.000 & -19.950 & 29.950 & 0.180 & 5.402 \\
\hline-2.000 & 1.000 & 0.100 & 10.000 & -20.000 & 30.000 & 0.180 & 5.404 \\
\hline-2.995 & 1.000 & 0.100 & 10.000 & -29.950 & 39.950 & 0.144 & 5.746 \\
\hline-3.000 & 1.000 & 0.100 & 10.000 & -30.000 & 40.000 & 0.144 & 5.748 \\
\hline
\end{tabular}

The crack growth rate is calculated in Table 4.4 based on the effective SIF data in Table 4.3. $C$ and $n$ are assumed to be $7.44 \mathrm{e}-10$ (in/cycle) $/\left(\mathrm{ksi}^{*} \mathrm{in}^{0.5}\right)^{n}$ and 3.4 , respectively. These values are taken from the curve fit to the intrinsic growth rate data for the AISI 9310 steel tests conducted in heated oil. $d a / d N$ as a function of $R$ is plotted in Figure 4.8.

The curve in Figure 4.8 shows that the crack growth rate in the negative $R$ regime is less sensitive to variations in $R$ compared to the positive $R$ regime. Between $R$ equal to zero and -3.00 , the crack growth rate varies by a factor of 1.96 . In a fatigue context, a difference of this order of magnitude is acceptable. As a result, one can conclude that when modeling fatigue crack growth, $\Delta K_{e f f}$, or likewise $K_{o p}$ or $d a / d N$, does not change significantly for $R \leq 0$. Therefore, the magnitude of $R$ is not a useful parameter to characterize damage evolution in gears. In the context of designing gear geometry to be damage tolerant, a primary concern need not be how aspects of gear geometry affect $R$.

It has been shown that when crack closure is taken into account there is not a significant change in the crack growth rates for negative $R$-values. This result will be utilized in the numerical analyses discussed in Chapters 5 and 7 . The load ratio will be taken as $R=0$ under the assumption that, if $R<0$, the general results and 
conclusions would still be valid. This is a simplification to the loading cycle and method.

Table 4.4: Crack growth rate calculations for a wide range of $R$-values taking into account crack closure effects. The percent change in $d a / d N$ is due to $\Delta R=0.005$.

\begin{tabular}{|r|c|c|c|}
\hline $\mathrm{R}$ & $\begin{array}{c}\Delta \boldsymbol{K}_{\text {eff }} \\
{\left[\mathrm{ksi}^{*} \text { in }^{0.5}\right.}\end{array}$ & $\begin{array}{l}\mathrm{da} / \mathrm{dN} \\
(\text { in/cycle } \\
\left.\boldsymbol{I 0}^{-7}\right)\end{array}$ & $\begin{array}{c}\text { \% Change } \\
d a / d N\end{array}$ \\
\hline 0.705 & 2.434 & 0.153 & -- \\
\hline 0.700 & 2.467 & 0.160 & 4.446 \\
\hline 0.505 & 3.542 & 0.549 & - \\
\hline 0.500 & 3.565 & 0.561 & 2.135 \\
\hline 0.255 & 4.388 & 1.136 & -- \\
\hline 0.250 & 4.399 & 1.146 & 0.873 \\
\hline 0.005 & 4.714 & 1.1449 & -- \\
\hline 0.000 & 4.716 & 1.451 & 0.130 \\
\hline-0.495 & 4.886 & 1.637 & -- \\
\hline-0.500 & 4.888 & 1.639 & 0.120 \\
\hline-0.955 & 5.058 & 1.841 & -- \\
\hline-1.000 & 5.060 & 1.844 & 0.116 \\
\hline-1.995 & 5.402 & 2.303 & -- \\
\hline-2.000 & 5.404 & 2.306 & 0.108 \\
\hline-2.995 & 5.746 & 2.841 & -- \\
\hline-3.000 & 5.748 & 2.844 & 0.102 \\
\hline
\end{tabular}

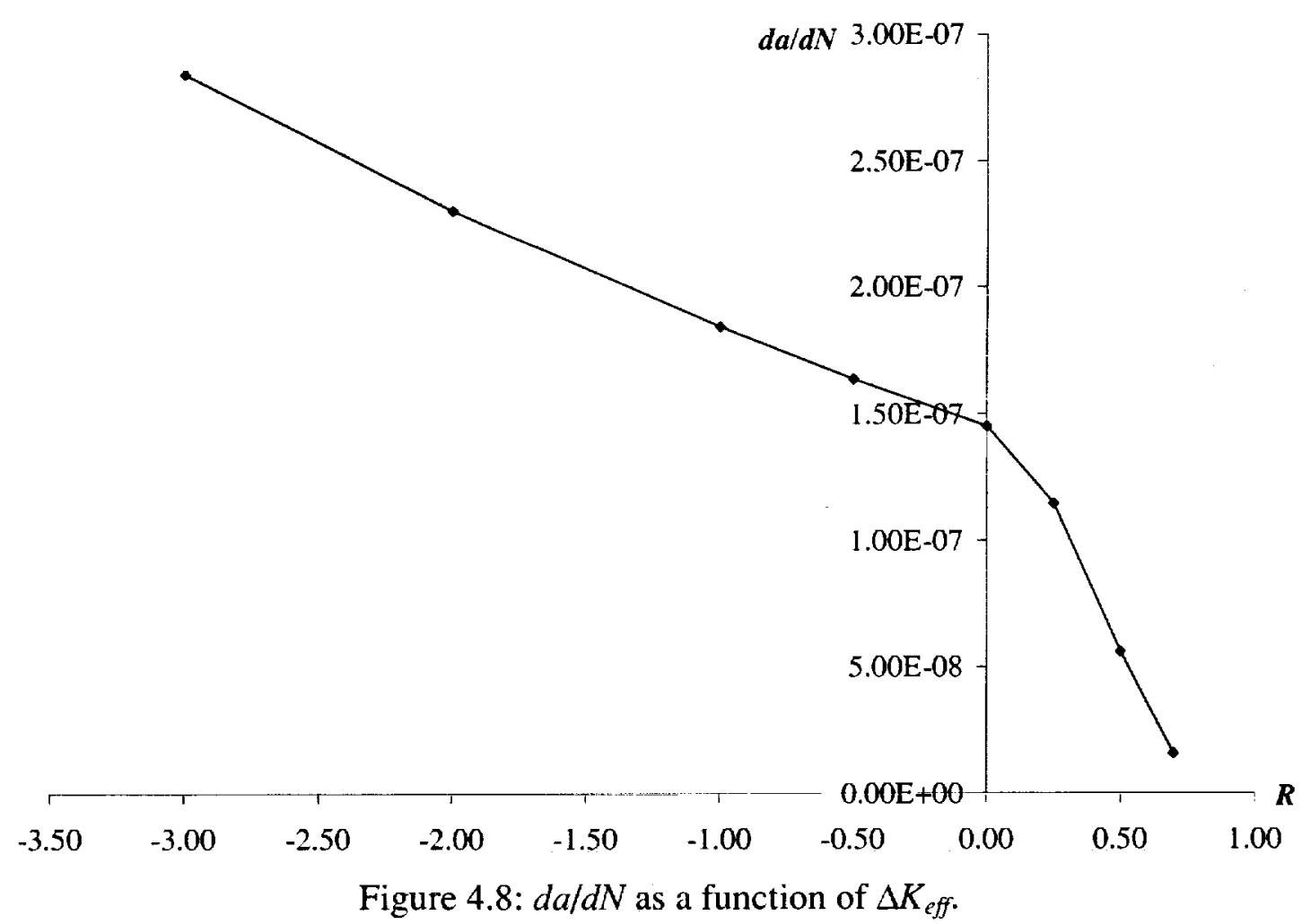




\subsection{Chapter Summary}

Highlights of this chapter can be summarized as follows:

- The crack closure concept and Newman's model were presented. It was shown that the model predicts that fatigue damage occurs only during the portion of the load cycle when the crack faces are not in contact.

- Newman's crack closure model was applied to empirical data for crack growth rates of a pressure vessel steel. It was shown that crack closure explains well the apparent dependence of crack growth rates on $R$. In fact, the material has an intrinsic crack growth rate. $R$ is a parameter that determines during what portion of the load range the crack faces are not in contact. This range is called the effective stress intensity factor range.

- Newman's model was applied to AISI 9310 steel, a typical steel used for gears. There was much less crack growth data available for this steel as compared to the pressure vessel steel. Nevertheless, it was shown that the crack closure model works for this small data set.

- It was demonstrated that, in the regime of negative $R$-values, the model predicts that the crack growth rates as a function of the effective stress intensity factors are only a weak function of the magnitude of $R$.

- The observation made in this chapter that crack growth rates are not highly sensitive to $R$ in the negative $R$-regime will be used in Chapter 5 when modeling the load history. 


\section{CHAPTER FIVE: PREDICTING FATIGUE CRACK GROWTH TRAJECTORIES IN THREE DIMENSIONS UNDER MOVING, NON-PROPORTIONAL LOADS}

\subsection{Introduction}

Chapter 5 covers numerical modeling issues related to predicting fatigue crack growth trajectories in three dimensions in a spiral bevel pinion. The goal of this chapter is to model crack growth under realistic operating conditions. As covered in Section 1.2, most previous work in the area of predicting crack trajectories in gears assumed one fixed load location. The location was usually the HPSTC. However, in operation, spiral bevel gears are subjected to a load moving in three dimensions. The fixed location loading, therefore, could lead to incorrect three dimensional trajectories.

A boundary element model of the $\mathrm{OH}-58$ spiral bevel pinion is presented in Section 5.2. The tooth coordinates and a dimensioned drawing of the pinion were provided by NASA/GRC, along with the coordinates for discrete elliptical contact areas along a spiral bevel gear tooth. OSM/FRANC3D is used to create the model from these data. Studies are conducted to determine the smallest model that still achieves accurate SIF results. Once this model is defined, initial analyses for the discrete load cases are conducted. The SIF history for an initial crack subjected to the moving load is presented in Section 5.3.

Section 5.4 develops a method to predict three dimensional fatigue crack growth trajectories under a moving load. The method increments a set of discrete points along the crack front for each step in the load cycle to find the total amount of extension and final angle of growth after fifteen load steps ( 1 load cycle). The propagation path for each point is then approximated with a straight line. A number of cycles are specified, and the crack front is advanced an amount equal to the crack extension for one cycle times the number of assumed cycles, and at the angle calculated for one cycle. Next, a curve is fit through the new crack tip locations to define the new crack front. The FRANC3D geometry model is updated, and the process is repeated.

Finally, in Section 5.5 the proposed moving load crack propagation method is implemented to predict fatigue crack growth trajectories in the OH-58 spiral bevel pinion.

\subsection{BEM Model}

A boundary element model of the $\mathrm{OH}-58$ spiral bevel pinion was built with OSM/FRANC3D. The Cartesian coordinates for a tooth surface, tooth profile, and fillet curve were provided by NASA/GRC. The data were generated automatically from a program that models the gear cutting process along with the gear kinematics. All points on the generated tooth surface are points of tangency to the cutter surface during the manufacturing process [Litvin 1991]. A primary motivation for developing the tooth geometry program was to generate data for a three dimensional finite element analysis. This program's output was adapted to develop a boundary element 
model for this thesis. The remainder of the pinion solid model was built from a drawing of the pinion. The basic shape of the shafts and gear rim were modeled. Some subtle details of the pinion drawing were ignored in cases where the geometry would complicate the geometry model and have negligible effects on the computed SIFs. The surfaces of the solid model were meshed using three- and four-noded elements. Figure 5.1 contains three views of a typical boundary element model (recall that the meshes shown in the figures are surface meshes). The volume of the gear is not meshed. The conical shape of the gear rim and the cylindrical shape of the shafts are seen best in Figure 5.1b. As seen in Figures 5.1a and 5.1c, three of the nineteen teeth of the pinion are modeled explicitly. Section 5.2.2 discusses studies to verify the accuracy of the three teeth model.

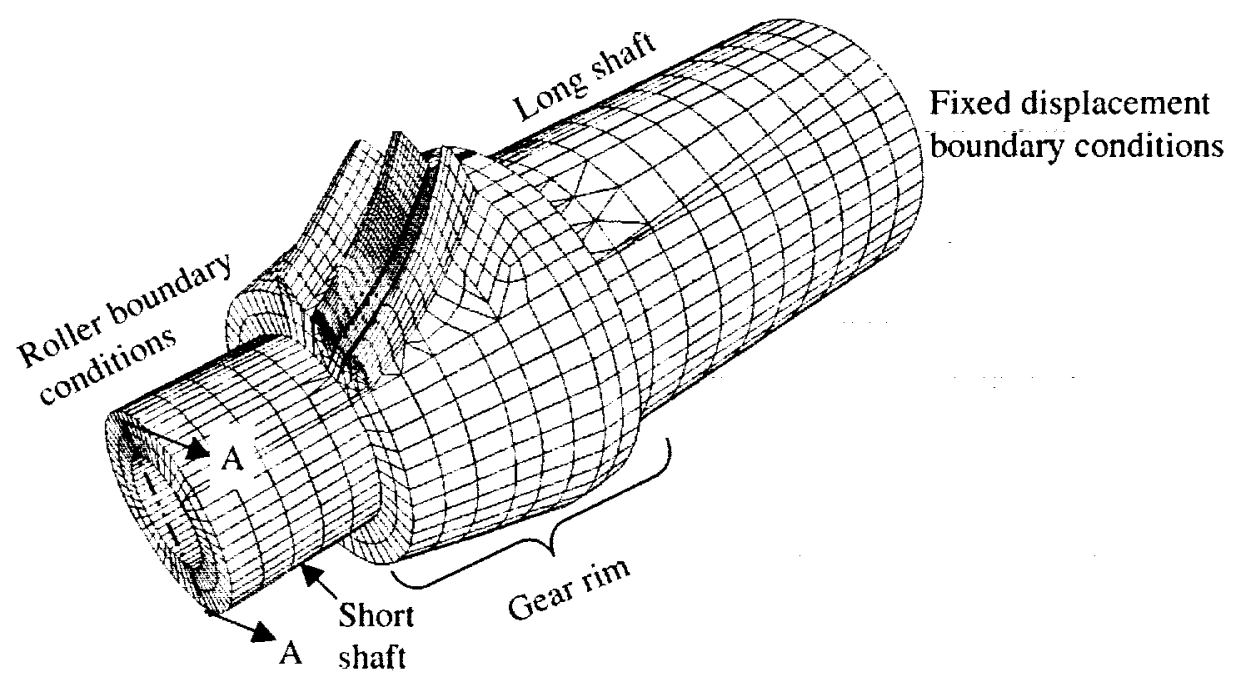

a) Overall view of full model

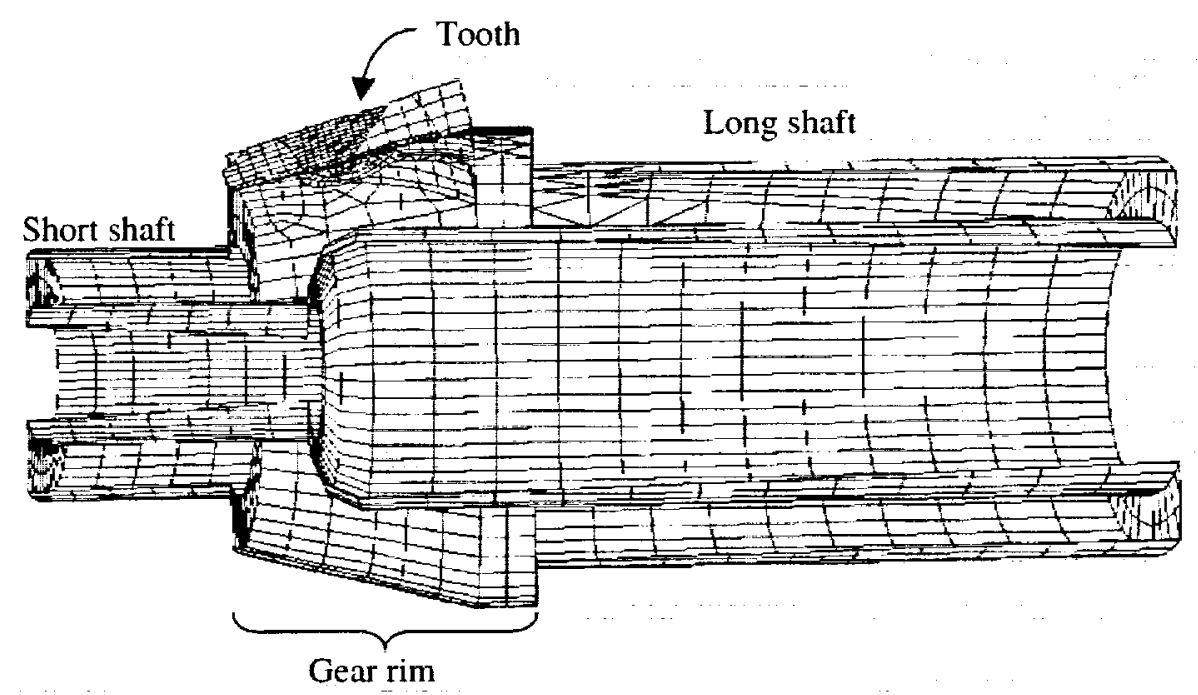

b) Section A-A from (a): profile of shaft 


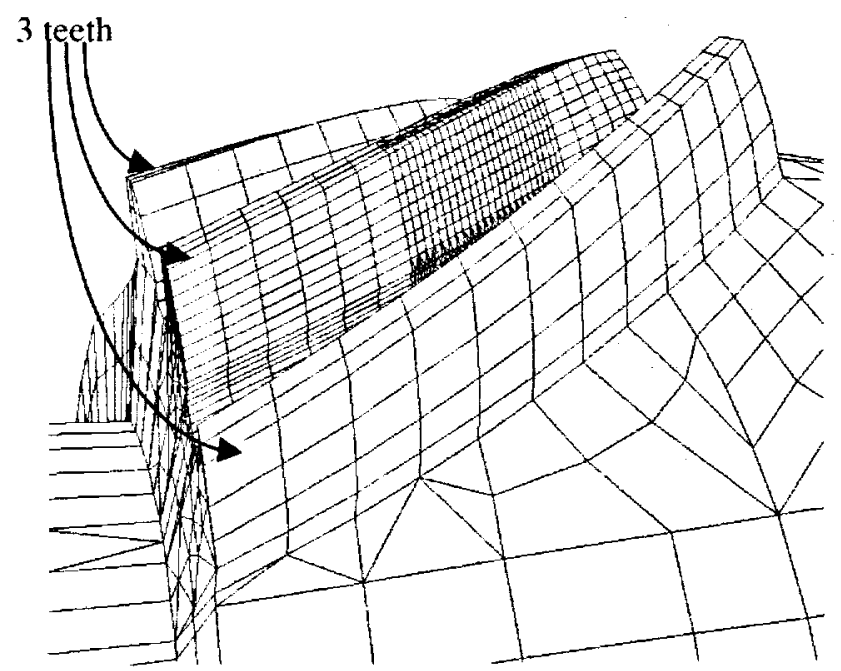

c) Close up view of teeth

Figure 5.1: Typical boundary element model of $\mathrm{OH}-58$ pinion.

In operation, the input torque is applied at the end of the pinion's long shaft. The small shaft sits on roller bearings. When the torque is applied, the gear rotates and the teeth of the pinion successively contact the gear's teeth. When contact occurs, load is transferred across the teeth. The boundary conditions shown in Figure 5.1 a model these operating conditions. This model will be referred to as the full model. The face patches at the end of the long shaft are fixed in all directions. The displacements on the surfaces of the smaller shaft are restrained in the local normal direction. Though not explicitly shown in Figure 5.1, contact areas are modeled as distinct face patches on the middle tooth. Traction normal to the patch is defined which equals the load that is transferred across the contacting teeth for a given input torque and rotation angle. More detail on how these contact patches are defined is given in the next section.

\subsubsection{Loading Simplifications}

The meshing of the gear and pinion is a continuous process. The magnitude of force between the gear teeth varies during the meshing as adjacent teeth come into and out of contact. Figure 2.8 is a schematic of the continuous process that has been discretized into fifteen load steps.

In order to perform numerical crack propagation studies of the pinion, the continuous contact between the teeth is discretized into fifteen contact patches, or load steps: four double tooth contact patches, seven single tooth contact patches, followed by four more double tooth contact patches. Each load step is a unique face patch in the boundary element model. The load steps will be referred to as numbers one through fifteen, corresponding to the patches from the gear root to the top land, respectively. This is consistent with the progression of contact area along a pinion tooth from the root toward the top. One progression through the fifteen load steps is one load cycle on the tooth. One rotation of the gear results in one load cycle on each tooth. 
The location and size of the fifteen discrete contact patches were provided by NASA/GRC. The data were determined numerically by a procedure similar to that described by Litvin et al. [1991]. The mean point of contact between the gears is taken as the center of the ellipse. Hertzian contact theory along with the applied torque level is used to determine the width of the ellipse. The patches were calculated for operating conditions of 300 horsepower, 6060 rotations per minutes, and 3120 in$\mathrm{lb}$ torque. These conditions are approximating the $100 \%$ design load condition, which is defined as $3099 \mathrm{in}-\mathrm{lb}$ torque.
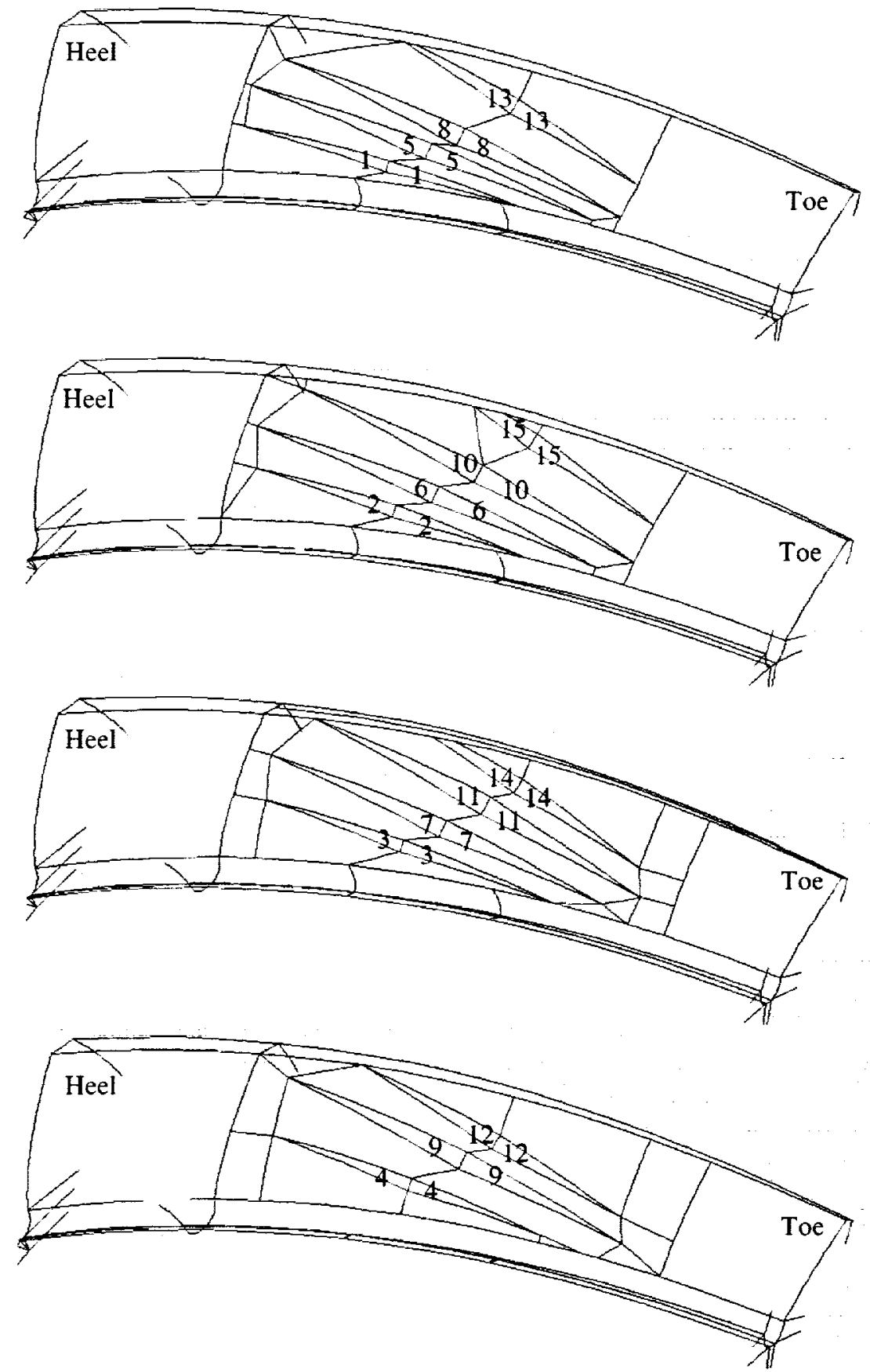

Figure 5.2: Contact ellipses defined at the geometry level in the numerical models. 
In the BEM model, the shape of a contact ellipse is approximated by straight lines connecting the axes' end points. The straight line approximation is valid because Saint Venant's principle holds; as long as the total applied forces and resulting moments are kept constant, the elliptical shape of the traction can be approximated by a patch with straight sides without altering the stress distribution along the crack front. Frictional forces are neglected, and, consequently, the traction is constant over the patch. Each patch has a unique magnitude of traction.

The four figures in Figure 5.2 demonstrate how the traction patches are built into the model geometry. The purpose of the models is to calculate SIFs from all fifteen static load cases. The combination of all fifteen SIF distributions represents one load cycle on the tooth. Figure 5.2 shows how a single BEM model can incorporate multiple load cases. Not all of the contact ellipses can be modeled in one BEM model because there is overlap between the ellipses. The multiple load case feature minimizes the computational time. For example, the boundary element model for load cases one, five, eight, and thirteen is virtually identical. The only difference between them is the boundary conditions. Hence, with the multiple load case feature, the two most computationally expensive steps of the boundary element solver, setting up the boundary integral equations and factoring the stiffness matrix, occur only once. The different boundary conditions are then applied individually, and the corresponding equations are solved for the unknown displacements and tractions for each load case.

\subsubsection{Influence of Model Size on SIF Accuracy}

The fewer the number of elements, or unknowns, in a boundary element model, the less computationally intensive the model is. Minimizing the number of elements can primarily be accomplished by 1) using a coarser mesh with larger elements or 2) by modeling less of the geometry of the solid. A disadvantage of the first option is the accuracy of the solution is sacrificed. The elements used in all of the studies in this thesis are linear. Therefore, only linear variations in displacement across an element can be represented. Likewise, the geometry is approximated by a series of linear segments. Because the geometry of the pinion is complex with significant amounts of curvature, larger elements do not represent the geometry adequately. As a result, this option is disregarded, and the second option, simplifying the model, is considered.

Simplifying the model also has drawbacks. The smaller the portion of the pinion modeled, the less accurate the representation of the boundary conditions. Three simplified models are investigated. The first simplification, Figure 5.3, is to ignore the long shaft in the full model. The new faces that are created when the long shaft is disregarded are restrained in all directions. Secondly, the smaller shaft is removed, Figure 5.4. The boundary conditions on the heel end are the same as simplification one, and the new faces on the toe end are set to traction free. The final simplification is to cut the rim of the pinion in half, Figure 5.5. The boundary conditions for this model are the same as the second simplification, with the addition of roller boundary conditions (displacement in the direction of the local normal set to zero) applied to the 
new faces. The boundary conditions for each model are chosen because they most closely match those of the full model (Figure 5.1a).

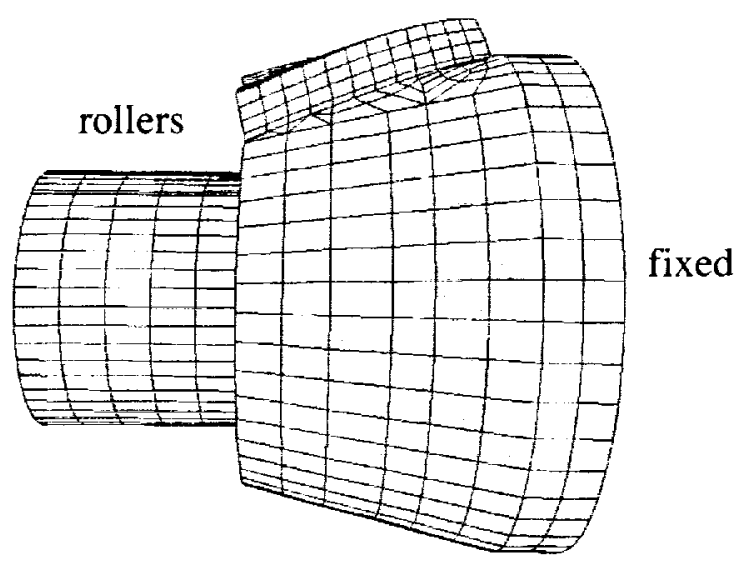

Figure 5.3: Simplified model 1: ignore long shaft.

In each of the simplified models, the flexibility of the pinion changes. When an identical crack is introduced into all of the models, the SIFs might vary from model to model. To determine whether a simplified model is valid, the SIFs from the simplified models are compared to the full model's SIFs for identical cracks. It is assumed that the full model most accurately represents the operating conditions and loading paths.

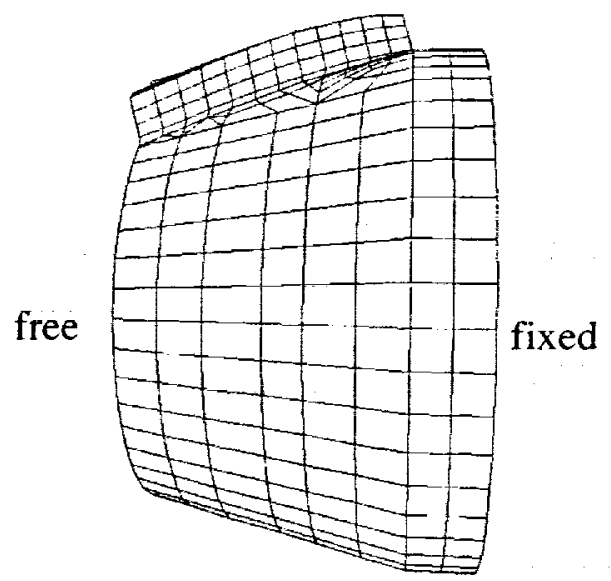

Figure 5.4: Simplified model 2: ignore both shafts. 


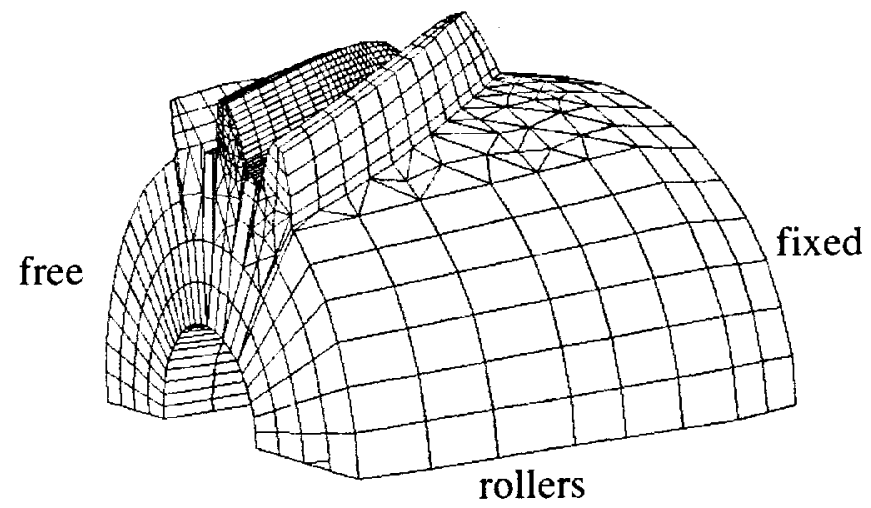

Figure 5.5: Simplified model 3: ignore both shafts and half of gear rim.

A semi-elliptical crack is introduced in the root of the middle tooth in each model. The crack is 0.64 inches long and 0.14 inches deep. A simplified load is applied to the middle tooth over the middle third of the tooth length and tooth height. The shape of the traction patch is rectangular, and the traction across the patch is constant. The shape and location of the traction patch is different from those described in Section 5.2.1. However, the difference is not important because the intent is to analyze differences in SIFs between models after changing one variable and keeping all the rest of the model parameters constant. To achieve consistency between all the models, the mesh in the region of the crack and load patch is identical.

The SIFs increase on average by $7 \%, 8 \%$, and $11 \%$ with respect to the full model's SIFs for simplification one, two, and three, respectively. In a fatigue growth rate context, changes of this magnitude are significant. Recall that the crack growth rate is proportional to $K_{I}$ raised to a power (Equation (3.6)). For AISI 9310, the magnitude of the exponent is approximately 3.4. Consequently, seemingly small changes in the SIFs have dramatic effects on the crack growth rate predictions. It is concluded from this study that the full model should be used for all trajectory predictions.

To verify that only explicitly modeling three teeth yields accurate results, a nine teeth model is analyzed. If the SIFs between the three teeth and nine teeth models are similar, then it can be concluded that not all of the nineteen teeth of the pinion need to be modeled.

An edge crack is introduced in the three and nine teeth models, in the middle of the tooth length, in the root of the concave side of the middle tooth. The crack shape is semi-elliptical, and is 0.125 inches long and 0.05 inches deep. An effort is made to keep the meshes between the two models identical.

The difference in SIF distribution under load steps 1,5 , and 8 is investigated. As shown in Figure 5.6, the percent difference in $K_{I}$ between the two models for all three load cases is below 5\%. The absolute magnitude of $K_{I I}$ for both models and all load cases is significantly smaller than $K_{I}$. Consequently, a small variation in $K_{I I}$ appears as a large percent difference between the models. Instead of percent differences, Figure 5.7 shows the absolute $K_{I I}$ values for all the loads and models. It is evident from the figures that the three teeth and nine teeth models yield similar results, 
leading to the conclusion that the three teeth model is sufficient for the trajectory prediction analyses.

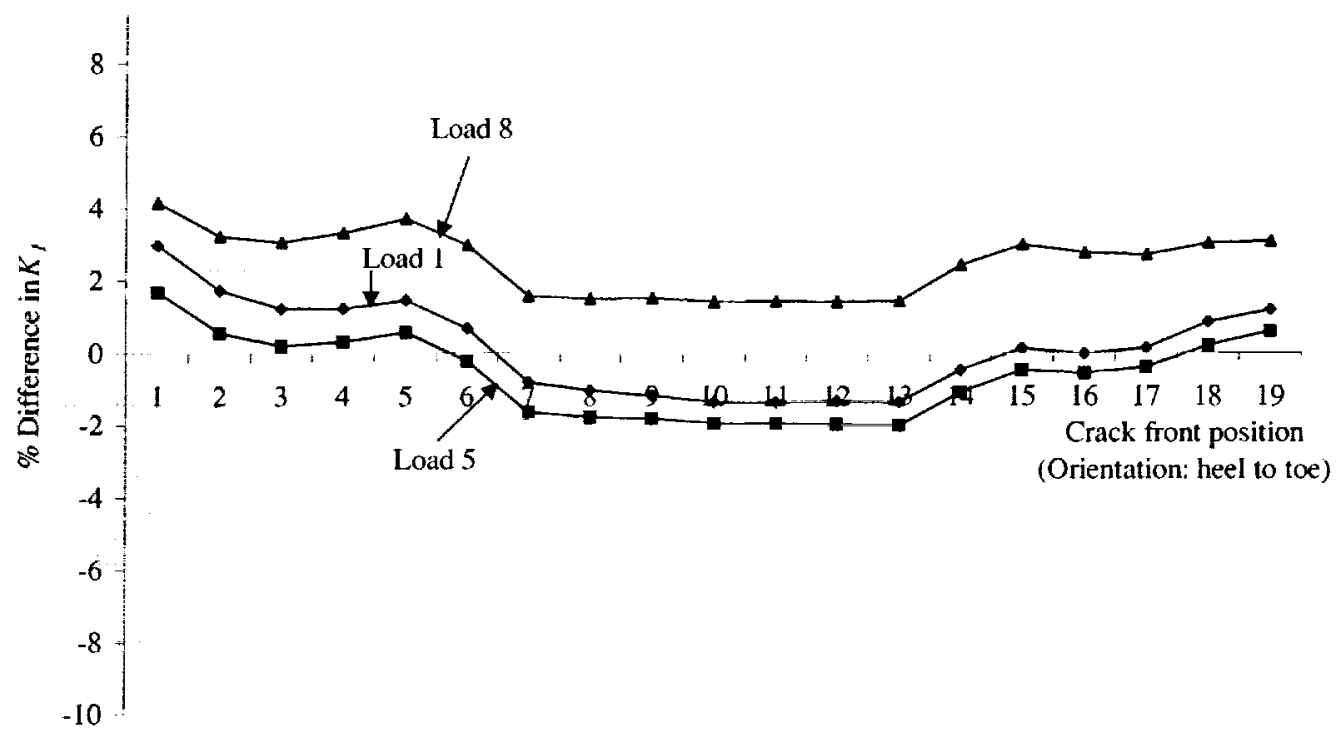

Figure 5.6: Percent difference in $K_{l}$ between three teeth and nine teeth models for load cases one, five, and eight. Crack front position one corresponds to the heel end of the crack front.

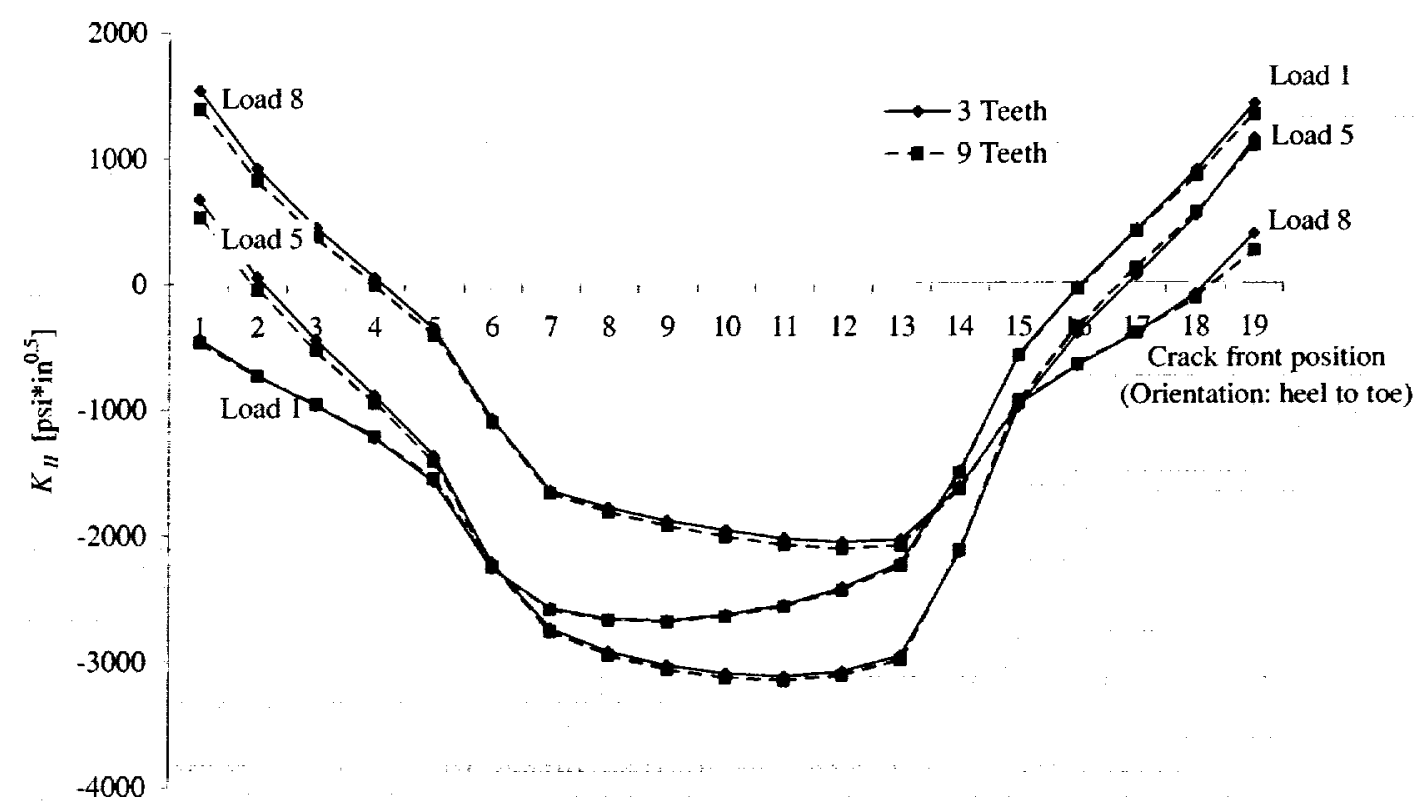

Figure 5.7: $K_{I I}$ distribution for three teeth and nine teeth models for load casses one, five, and eight.

\subsection{Initial SIF History Under Moving Load}

To simulate the moving load during one load cycle on a pinion's tooth, fifteen static BEM analyses are performed. Each analysis represents one of the fifteen 
discrete time steps as the contact area moves up the pinion tooth, as discussed in Section 5.2.1. Recall these contact ellipses are defined for a full design load input torque of $3120 \mathrm{in}-\mathrm{lb}$. The full pinion boundary element model is used, Figure 5.1. A semi-elliptical edge crack is introduced into the root of the middle tooth on the concave side. The crack is located approximately in the middle of the tooth length. The dimensions are 0.125 inches long by 0.050 inches deep. The crack is oriented approximately normal to the surface.

Each load step produces a unique SIF distribution along the crack front. The SIF distribution changes between load steps because the load position and magnitude varies from step to step. Figure 5.8 shows the mode I SIF distribution for the first eleven load steps, the initial four double tooth contact load steps followed by the seven single tooth contact load steps. The second stage of double tooth contact, load steps twelve through fifteen, are omitted from the figure to simplify it. Modes II and III SIFs are plotted similarly in Appendix A.

The four bottom curves in Figure 5.8 are the SIFs under double tooth contact. The remaining seven curves are the SIFs under the single tooth contact load steps. The bottom most of the seven curves corresponds to load five. The topmost curve is the result from load eleven, the last single tooth contact step. The total applied force for each single tooth contact load step is roughly equivalent. However, as the load step number increases, the SIF curves shift up. This is explained by the fact that the locations of the contact patches are progressing up the pinion tooth. The change in location creates a greater moment arm. As a result, the displacements, and likewise SIFs, in the tooth root will thus increase.

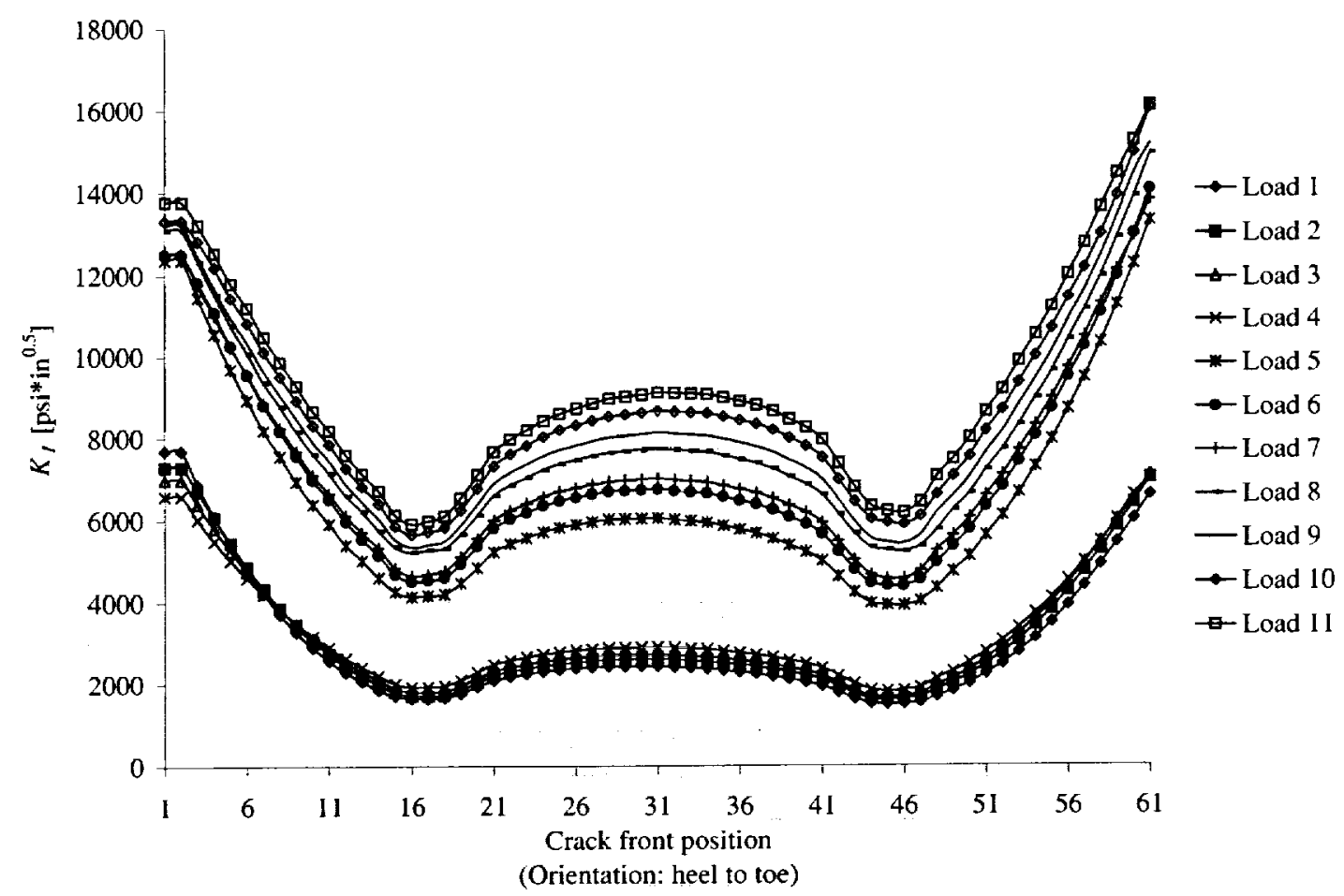

Figure 5.8: Mode I SIF distribution for load steps one through eleven. 
Another approach to examine the data is to plot the SIF history for each point along the crack front. Figure 5.9 shows the SIF history for point 29 in Figure 5.8 (roughly the midpoint of the crack front). The magnitude of $K_{l}, K_{I l}$, and $K_{I I}$ is plotted as a function of time, or load step. The figure also includes $K_{o p}$, which was calculated using Newman's crack closure equations described by Equations (4.1), (4.2), (4.4), and (4.5).

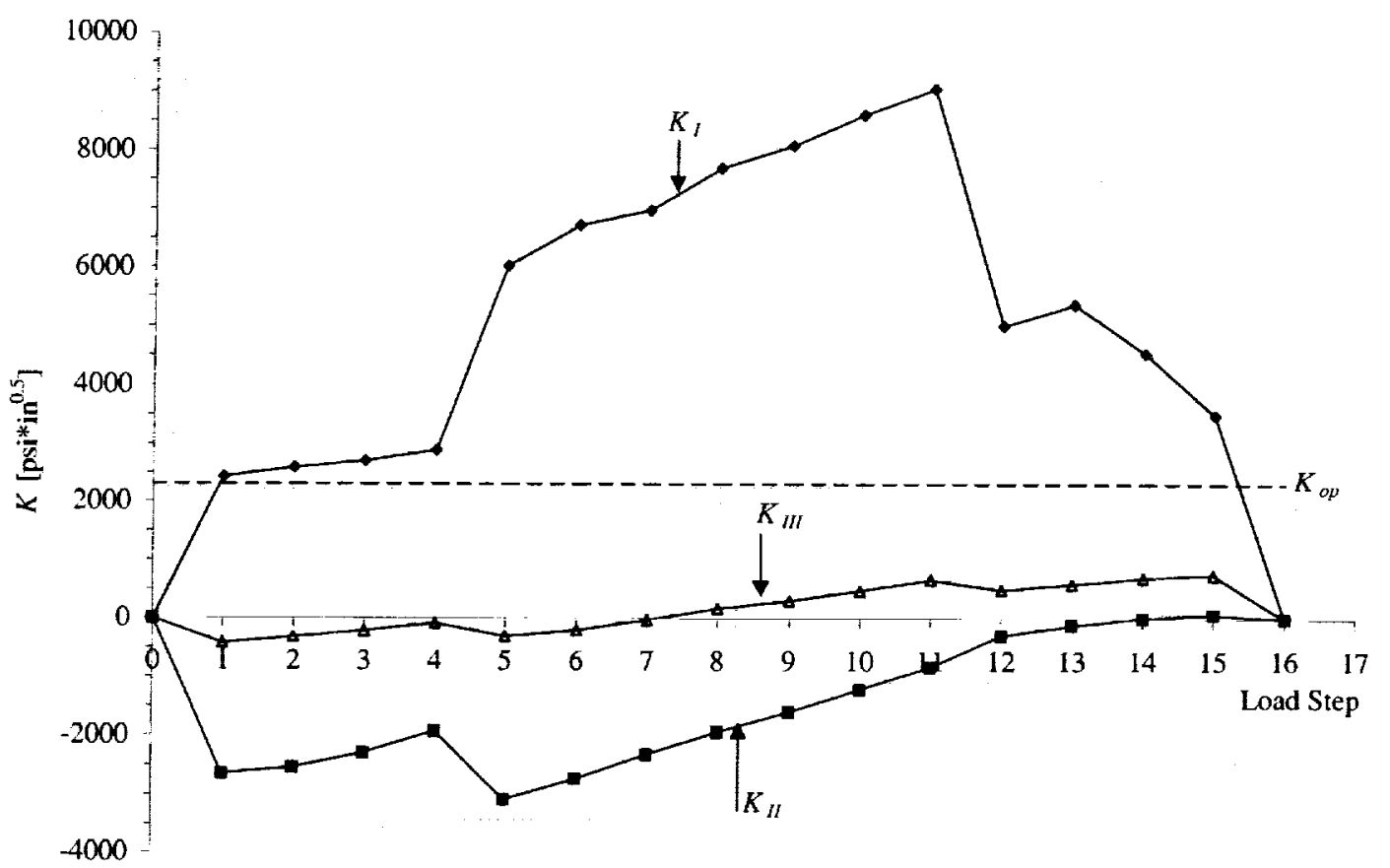

Figure 5.9: Typical SIF history for one load cycle for one point on crack front.

When the individual points in Figure 5.9 are connected with straight lines, the plots represent the loading cycle on the tooth. The minimum load has been taken to be zero. In actuality, the minimum load in the tooth root might be compressive. When a tooth is loaded, compressive stresses could result in the root of the convex side. Depending on the magnitude of these stresses, they may extend into the concave root of the adjacent tooth. However, Chapter 4 demonstrated that the crack growth rates do not vary significantly for negative $R$-values when crack closure is taken into account. Therefore, the load cycle is modeled as $R$ equals zero, i.e. $K_{\text {Imin }}=K_{\text {IImin }}=K_{\text {IIImin }}=0$.

The difference in the single tooth and double tooth contact loads is evident in the mode I SIFs. The plateaus in the curve correspond to the two contact stages. $K_{l}$ is significantly larger during single tooth contact (load steps 5-11) compared to the double tooth contact stages (load steps 1-4 and 12-15). The magnitudes of $K_{I}$ are significantly greater than $K_{I I I}$. As a result, it will be assumed that mode III does not contribute to the crack growth.

Based on gear theory, the curves in Figure 5.9 should be continuous and smooth. The continuous curves would most likely show a large increase in slope as the loading progresses from double tooth contact to single tooth contact. One can 
imagine that as the number of discrete load steps increases, the curves in Figure 5.9 will become smoother. However, due to transmission error and noise, it is known that the curves in reality are neither continuous nor smooth. Therefore, it is assumed that the fifteen load steps are sufficient to approximate the true loading conditions.

The moving load on the pinion's tooth is non-proportional; the ratio of $K_{I I}$ to $K_{I}$ changes during the load cycle, Figure 5.10. Consequently, the method to propagate a three dimensional crack described in Section 3.2.3 can not be used. That method assumed proportional loading, which results in a constant kink angle for the load cycle. Since the ratio is changing in the spiral-bevel gear tooth, the predicted angle of propagation during the load cycle changes. A method to determine how a crack would grow under this type of loading is required and is proposed in Section 5.4.

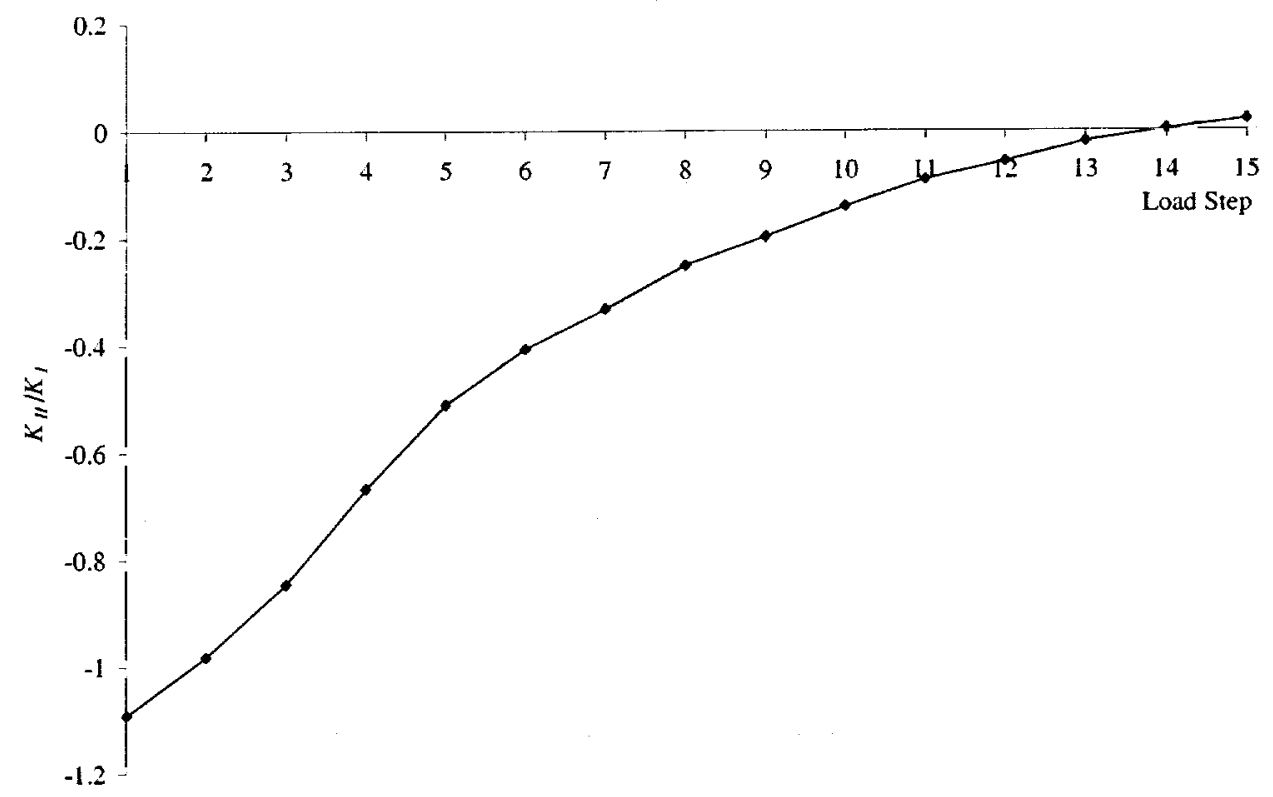

Figure 5.10: Typical $K_{I I}$ to $K_{I}$ ratio under moving load.

The $K_{I I}$ to $K_{I}$ ratio also indicates which loading mode is driving the crack growth. Mode I dominant fatigue crack growth is associated with smaller ratios. Qian et al. [1996] studied mixed mode I and II crack growth in four point bend specimens. They selected the test specimen geometries from FEM analyses that considered various crack lengths and orientations to achieve different $K_{I I}$ to $K_{I}$ ratios. From the analyses, they selected five different geometries with $K_{I I} / K_{I}$ values of $0,0.262,0.701$, 1.812 , and 16.725 . The ratios covered crack growth mechanisms of pure mode I, mode I dominant, balanced mode I and II effects, mode II dominant, and highly mode II dominant, respectively. Using these ratios as guidelines, the gear situation can be characterized as balanced mode I and II effects during the earlier stages of the cycle to mode I dominant crack growth during the later stages of the cycle. However, it will be assumed that the fatigue crack growth is driven by mode $\mathrm{I}$. 


\subsection{Method for Three Dimensional Fatigue Crack Growth Predictions Under Non-Proportional Loading}

As shown in Section 5.3, a crack in a spiral bevel pinion tooth is subjected to non-proportional loading. As a result, conventional methods to predict crack growth trajectories in three dimensions are not adequate. A literature review of nonproportional fatigue crack growth revealed only a few methods that were applicable to the gear model; Section 5.4.1 is a summary of relevant work. A method to predict three dimensional fatigue crack trajectories under non-proportional loads is proposed in Section 5.4.2. Section 5.4.3 summarizes the approximations of the method.

\subsubsection{Literature Review}

In the literature related to non-proportional fatigue crack growth, the majority of the work is experimental. The limited amount of numerical work is related to predicting crack growth rates and fatigue life. The numerical work is also largely confined to two dimensional analyses. Schijve [1996] gives an overview of methods and research related to predicting fatigue life and crack growth. There is no mention of predicting crack trajectories in non-proportional loading scenarios. Crack trajectories are of primary importance in the context of gears because the trajectory determines whether the failure mode will be catastrophic. The number of cycles to failure is of secondary importance because the high loading frequency on a gear's tooth results in very short times from crack initiation to tooth or rim failure.

Bold et al. [1992] is the most extensive report covering fatigue crack growth in steels under mixed mode I and $\Pi$ loading. They give experimental results from nonproportional mode I and II tests, and compare the maximum tangential stress theory (pure mode I) and maximum shear stress (pure mode II) theory for predicting kink angles to experimental results. However, their work contains no theoretical predictions for mixed mode. Bower et al. [1994] considered brittle fracture under a moving contact load. They incrementally advanced the load and evaluated the SIFs at each stage of contact. If the SIFs met their fracture criterion, then the crack was propagated based on the mode I and II SIFs for that load position using the maximum principal stress criterion. Their approach incorporates non-proportional loading in an incremental manner; however, the work is limited to brittle fracture, does not include fatigue, and does not include three dimensional effects.

Hourlier et al.'s [1985] focus was to determine which of three theories predicted trajectories closest to experimental data for non-proportional loading. They worked in terms of $k_{l}$, which is the mode I stress intensity factor for a small advance of the crack at an angle $\theta$. The three theories investigated were 1) direction in which $k_{l}$ is a maximum, 2) direction where $\Delta k_{l}$ is a maximum, and 3) direction of maximum fatigue growth rate $d a / d N$. The rate is calculated assuming a mode I dominant growth mechanism and is a function of $k_{I \max }(\theta)$ and $\Delta k_{I}(\theta)$. Their work found that 1) was the most inaccurate method. In general, the maximum $d a / d N$ method was found to best match experimental results. Hourlier et al.'s work is not practical for the purposes of this thesis primarily because it requires one to express the moving load and $k_{l}$ in closed forms as functions of time and $\theta$ in order to find the angle corresponding to the maximum $d a / d N$. 
Three dimensional finite element analyses have been performed to simulate the wheel position over a railroad track containing a crack [Olzak et al. 1993]. The rail model is analyzed for consecutive stages of wheel position and the SIFs are calculated for each stage. However, Olzak et al. did not propagate the crack. Their primary goal was to determine what happens to the crack displacement and contact shape when the load is directly over the crack. In the case of the spiral bevel gear, the load will most likely never be directly over the crack and their findings are not applicable.

The most significant work was done by Panasyuk et al. [1995]. They numerically modeled and propagated a two dimensional edge crack under a moving contact load. The maximum principal stress theory was used, and growth rates were calculated by Paris' model. The translation and location of the contact are expressed as functions of $\lambda$, the distance from the load to the crack. To calculate the kink angle, first the values of $\lambda$ that correspond to an extremum of $K=F\left[K_{l}(\lambda), K_{I I}(\lambda), \theta(\lambda)\right]$ are found. Next $\theta, K_{l}$, and $K_{I l}$ at these $\lambda$ are calculated, from which the growth rate is calculated. Finally, it is assumed that the crack propagates for $N$ cycles at that growth rate and angle, and the crack in the numerical model is updated and the process is repeated. Panasyuk et al. assumed that their geometry was an elastic half plane, and, therefore, they could set up closed form equations and solve analytically for $K_{l}, K_{I I}$, and $\theta$. Once again, their method can not be directly applied to gear model because neither the traction nor the geometry can be expressed in closed form. The method also does not directly take into account the non-proportional loading and assumes a constant kink angle for the entire load cycle. However, their method is extended and a similar incremental approach is developed in the next section.

\subsubsection{Proposed Method}

Compared to a two dimensional static problem, the problem at hand is continuous in time and in a third space dimension. Methods have been presented in previous chapters and sections to discretize both of these dimensions. With the discretizations, two dimensional crack propagation theories can be applied. In summary, the proposed method discretizes the continuous loading in time into a series of elliptical contact patches, or load increments. Two dimensional fatigue crack propagation theories are then used to propagate incrementally a series of discrete points from the three dimensional crack front. The remainder of this section outlines a proposed method to predict fatigue crack trajectories in three dimensions taking into account time varying SIFs.

\section{Method}

1. Discretize tooth contact path into 15 load steps (Section 5.2.1).

2. Calculate by the displacement correlation method, using a feature built in to FRANC3D/BES, the mode I, II, and III SIFs $\left(K_{I(j)}^{i}, K_{I I(j)}^{i}, K_{I I I(j)}^{i}\right)$, where $i$ is a discrete point along the crack front $(i=1-n u m$ points $)$ and $j$ is the load case $(j=0-M)$. In general, the nodes of the first row of mesh nodes behind the crack front are taken as the discrete points. Figure 5.9 is a typical plot of the SIFs for a single point $i$ along the crack front for the entire loading cycle. 
3. The goal of this step is to calculate for a given point $i$ the amount of crack extension, $d a_{(j-1, j)}^{i}$, during a load step from $j-1$ to $j \cdot \theta_{(j-1, j)}^{i}$ is the angle for the extension during a load step from $j-1$ to $j$. It is assumed that the crack grows incrementally during a load cycle. In addition, propagation at point $i$ only takes place when the change in mode I SIF between load steps is positive, $\left(K_{I}{ }^{i}-K_{I}{ }^{i}(j-1)\right)>0$, and only when $K_{I j}^{i}$ is greater than the opening SIF at that point, $K_{o p}{ }^{i}$. This implies that growth will only take place during the loading portion of the cycle.

To calculate most accurately the total amount of crack growth over one cycle, crack closure is taken into account. The amount of extension during one load cycle predicted by a modified Paris' model, adjusted to incorporate crack closure, is

$$
d a^{i}=C\left(\Delta K_{\text {eff }}^{i}\right)^{n}
$$

where $\Delta K_{\text {eff }}^{i}=K_{I \text { max }}^{i}-K_{o p}{ }^{i}=U * K_{l \text { max }}^{i} . U$ is given by Equation (4.2). $K_{o p}{ }^{i}$ is found using Equations (4.4) and (4.5). Figure 5.9 shows that the loading is characterized by $R=0$.

In order to calculate $U, S_{\text {max }}$, the far field stress in a Griffith crack problem, is required. Figure 5.11 shows the Griffith crack geometry [Griffith 1921]. The gear's geometry is obviously different from a Griffith crack problem. Therefore, an equivalent $S_{\max }{ }^{i}$ must be calculated for the gear. First, $K_{I \max }^{i}$ is found in step 2. $S_{\text {max }}{ }^{i}$ is then found by solving Equation (3.2) for $S_{\text {max }}{ }^{i}$ :

$$
S_{\max }^{i}=\sigma_{\max }{ }^{i}=\frac{K_{I}{ }^{i} \max }{\beta \sqrt{\pi a}}
$$

Lastly, it is assumed that, at a given point, the amount of extension between load steps is proportional to the ratio of the change in mode I SIF to the effective SIF. The amount of crack growth for each load increment is given by:

$$
d a^{i}{ }_{(j-1, j)}=\frac{K_{I}{ }^{i}(j)-K_{I}{ }^{i}(j-1)}{\Delta K_{\text {eff }}{ }^{i}} d a^{i}
$$

The angle of crack growth associate with each load increment is found from the maximum principal stress theory using the current load steps SIFs as:

$$
\theta^{i}{ }_{(j-1, j)}=2 \tan ^{-1}\left[\frac{1}{4} \frac{K_{I}^{i}(j)}{K_{l l}{ }^{i}(j)} \pm \frac{1}{4} \sqrt{\left(\frac{K_{I}{ }^{i}(j)}{K_{l l}{ }^{i}(j)}\right)^{2}+8}\right]
$$




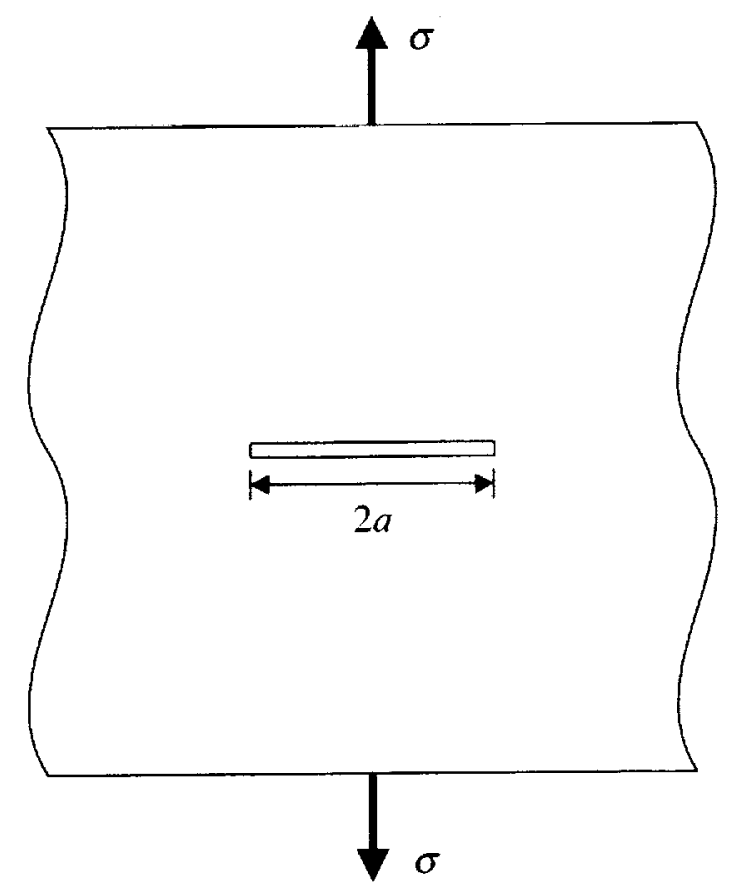

Figure 5.11: Griffith crack problem: Straight, through thickness crack in an infinite plate subjected to uniform tensile stresses [Griffith 1921].

4. Repeat step 3 for every load step of the cycle to get the final coordinates and angle for the trajectory during one load cycle. The final crack trajectory is approximated by a straight line from the initial crack tip location to the final crack growth location. Based on simple geometry, the final length, $d a_{T}^{i}$, and final angle, $\theta_{f}^{i}$, after one load cycle are calculated in the following manner:

$$
\begin{aligned}
d a_{T}{ }^{i} & =\sqrt{\left(l^{i}\right)^{2}+\left(h^{i}\right)^{2}} \\
\theta_{f}{ }^{i} & =\tan ^{-1}\left(\frac{h^{i}}{l^{i}}\right) \\
\text { where } \quad l^{i} & =\sum_{j=1}^{M} d a_{(j-1, j)}^{i} \cos \left(\theta_{(j-1, j)}^{i}\right) \\
h^{i} & =\sum_{j=1}^{M} d a_{(j-1, j)}^{i} \sin \left(\theta_{(j-1, j)}^{i}\right)
\end{aligned}
$$

Figure 5.12 illustrates this step schematically, assuming the load cycle has been discretized into four steps, i.e. $M=4$. Note that the arc length, which is the sum of the $d a_{(j-1, j)}^{i}$, is equal to the amount of growth predicted by the crackclosure-modified Paris model, Equation (5.1). The arc length is given mathematically by

$$
d a^{i}=\sum_{j=0}^{M} d a^{i}(j-1, j)
$$

5. Repeat steps 3 and 4 for every point along the crack front. 
6. Determine the number of cycles, $N$, necessary to achieve a significant amount of crack growth in relation to the pinion's geometry. This step is necessary because the amount of crack growth over one load cycle is too small to update the geometry model. Therefore, it is necessary to assume that a series of load cycles has occurred prior to changing the geometry. Because each point along the crack front has a unique growth rate associated with it, the crack front will not grow uniformly. Each point will grow by an amount $d a_{\text {final }} i$.

$$
d a_{\text {final }}{ }^{i}=N * d a_{T}{ }^{i}
$$

In general, $N$ is chosen such that $d a_{\text {final }} i \geq 0.01$ inches.

7. Update the FRANC3D geometry model with the new crack that has grown by an amount of $d a_{\text {finul }} i$. To accomplish this, a least squares curve fit is performed through the new discrete crack front points. A single polynomial curve may be fit through all of the points, or the points may be divided into a user-defined number of sets and individual polynomial curves are fit to each set. After the crack geometry is updated, it is necessary to locally remesh the model prior running the BEM solver. Once again, all load steps are analyzed with the new crack.

8. Repeat process beginning at step 2 .

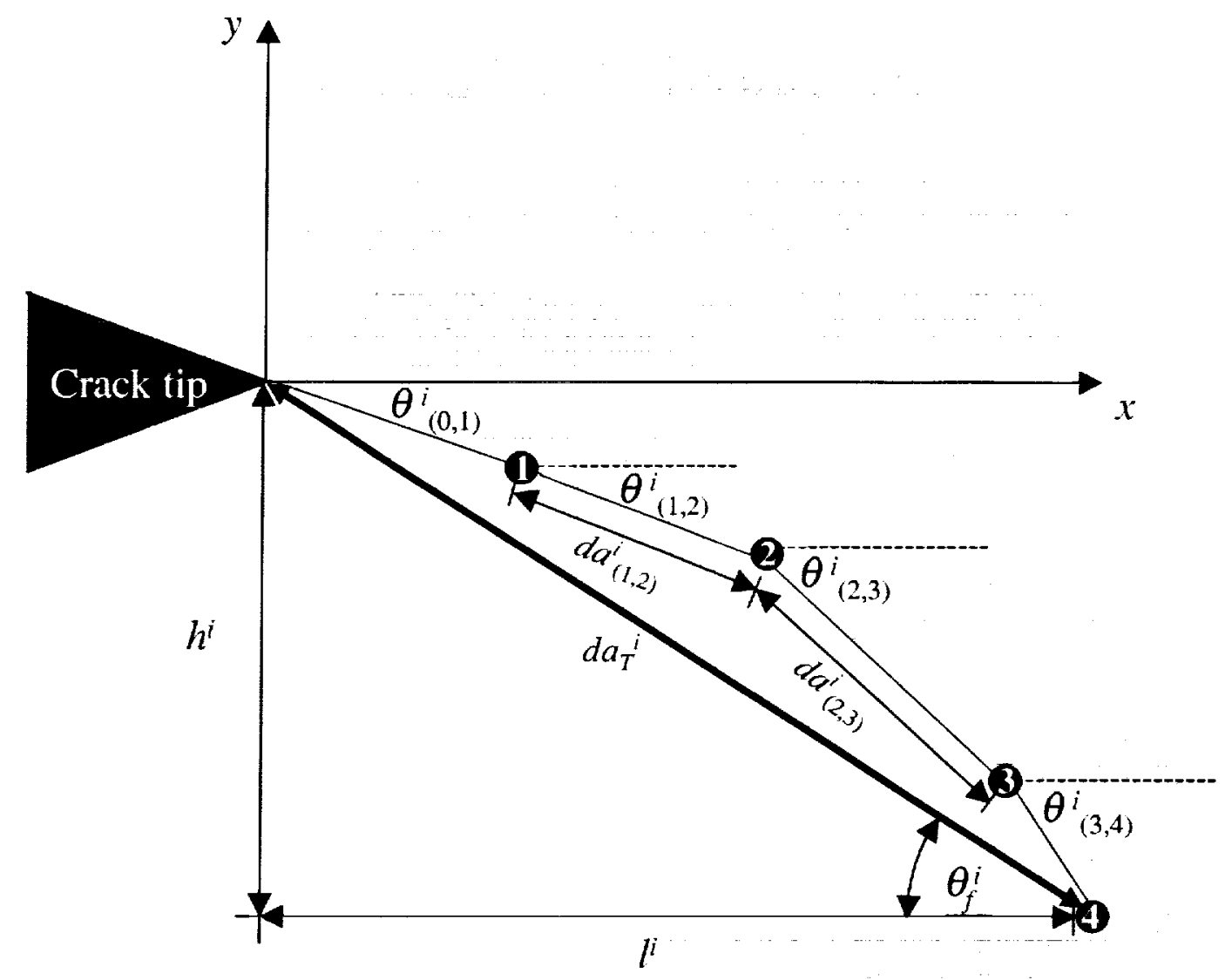

Figure 5.12: Schematic of crack extension for one point along the crack front after one load cycle. 


\subsubsection{Approximations of Method}

The accuracy of the proposed method is limited by the accuracy of the BEM results. In addition, several approximations and simplifications in the loading conditions and crack growth rate models could affect the accuracy. The shape of the elliptical contact patches is estimated by straight lines. When the applied traction is far enough away from the crack front such that the deformation due to local contact stresses does not contribute to the crack tip field, St. Venant's principle holds. In this case, the shape of the traction area is inconsequential and the straight line approximation is legitimate. However, if the crack trajectory is significantly close to a contact patch, then this assumption is no longer valid. As a result, the accuracy of the SIFs could be comprised. Furthermore, the size and location of each of the fifteen contact areas are kept constant throughout the crack propagation. In reality, the change in flexibility of the tooth could change the contact areas and in turn affect the SIF distribution in the later stages of propagation. This aspect will be investigated in Chapter 7. However, if the same contact areas are used during the entire crack propagation simulation, the fatigue life predictions will most likely be conservative. This is because the SIFs continuously increase as the crack advances when the loading scenario is kept constant and the crack length is increasing.

It is assumed that the crack front conditions are characterized by plane strain along the entire front. This is consistent with the maximum principal stress theory equations implemented to calculate the kink angle. However, shallow çracks and portions of edge cracks near the free surface are usually characterized by plane stress, not plane strain. Nevertheless, crack growth rates will be larger in plane strain conditions than plane stress. This assumption errs on the conservative side and will predict shorter fatigue lives. The size of the plastic zone in the gear is investigated more in Section 7.3.2.

An additional approximation is introduced when incrementally calculating the amount of crack growth. The method assumes that crack growth only occurs during the tensile portion of the load cycle. However, it can not be experimentally shown that this is true. In fact, it is generally accepted that crack growth occurs during the opening and closing portion of the load cycle [Laird 1967]. The tensile portion creates plastic deformation at the crack tip and causes it to blunt. During unloading, the plastic deformation creates a wedging action at the crack tip that acts to advance the crack.

The reasoning behind Equation (5.3) governing the crack growth rate is that, if the loading were to become proportional, the amount of crack extension predicted during one loading cycle would be equal to that predicted by the crack-closuremodified Paris' model. An additional assumption is that the method assumes mode I dominant fatigue crack growth. If the ratio of $K_{I I}$ to $K_{l}$, and likewise the ratio of $K_{I I I}$ to $K_{l}$, were to become large enough, the mode II (or mode III) loading could contribute to the crack growth. In this case, $\Delta K_{\text {eff }}$ should be a function of $K_{l}, K_{l l}$, and/or $K_{I I I}$. The models proposed for crack growth rates do not incorporate the mode II and III effects.

For each propagation step, a value for $N$ is chosen that is large enough to increase a majority of the crack front a significant distance in relation to the model's geometry. It is assumed during the $N$ loading cycles that the variations in the 
displacements and stresses are negligible. In reality, the crack is growing slowly during the cycles and therefore the displacements and stresses at the crack front will vary as the crack advances. If the value of $N$ is too large, then the model will not pick up subtle changes in displacements. Consequently, the predicted crack front shape and trajectory will be inaccurate.

\subsection{Simulation Results}

Three dimensional fatigue crack trajectory simulations are performed using the method described for non-proportional loading in Section 5.4.2. The full pinion boundary element model is used and an initial crack is introduced into the model as described in Section 5.3. In order to validate the predictions, the dimensions and shape of the crack are similar to a starter notch that was used in a tested pinion. The experimental results and data are reported in Chapter 6.

Thirteen crack propagation steps are carried out, which model the crack at thirteen distinct stages of growth. In each propagation step, the SIFs are evaluated using the displacement correlation method at the mesh nodes in the first row of elements along the crack front. To reduce the computational time for each propagation step, the second stage of double tooth contact, load steps twelve through fifteen, are ignored. The method for propagation adopted here only allows crack growth during loading. The final four load steps represent the unloading portion of the load cycle and, therefore, do not contribute to crack growth in the simulations.

The Paris growth rate model modified to incorporate crack closure is used in this study. The model parameters are held constant during the propagation steps. Values for $C$ and $n$ are taken from a curve fit to AISI 9310 steel crack growth rate data from $250^{\circ}$ oil (Section 4.4). $\kappa$ is set equal to three (plane strain). To calculate $S_{\max }{ }^{\prime}$, which is a variable in the $K_{o p}$ calculation, $\beta$ is set to one. The material properties listed in Table 2.2 are used.

A least squares curve fit to the predicted discrete crack front points is used to determine a smooth crack front curve. The approach is to fit a polynomial curve of second or third order to groups of points. To allow unsymmetric crack front shapes, the crack front points are divided into one, two, or three groups. A curve is then fit independently through each group.

The tooth contact locations and magnitudes defined for the $100 \%$ design load are used throughout the thirteen propagation steps. The loading simplifications for these data were presented in Section 5.2.1.

Table 5.1 contains the crack geometry and growth rate data for each of the propagation steps. $N$ is rounded to the nearest 100 cycles. Figures 5.13 and 5.14 show the initial and final crack trajectories along the tooth surface and the depth of the crack into the gear rim. At the end of propagation step thirteen, the crack has propagated neither entirely through the tooth width nor through the rim. The analyses are stopped at this step because the toe end of the crack has reached the top land. It was decided that continuing the analyses would lead to no additional insights because, as reported in Chapter 7, the prediction differed from the experimental results. Also, one can imagine that if the simulations were continued, since the trajectory has turned on both the heel and toe end, this middle portion of the tooth will break away from the gear. 
Table 5.1: Crack propagation data from trajectory prediction.

\begin{tabular}{ccccc}
\hline $\begin{array}{c}\text { Propagation } \\
\text { Step }\end{array}$ & $\begin{array}{c}N \\
\text { [cycles] }\end{array}$ & $\begin{array}{c}\text { Crack Front } \\
\text { Length [in] }\end{array}$ & $\begin{array}{c}\text { Depth } \\
{\left[\text { in } ~ 10^{-2}\right]}\end{array}$ & $\begin{array}{c}\text { Area } \\
\left.\text { [in }^{2} \times 10^{-2}\right]\end{array}$ \\
\hline 0 & N.A. & 0.200 & 5.00 & 0.579 \\
1 & 15,000 & 0.233 & 5.53 & 0.743 \\
2 & 20,200 & 0.258 & 5.58 & 0.837 \\
3 & 38,200 & 0.237 & 5.17 & 0.933 \\
4 & 56,200 & 0.453 & 5.88 & 1.69 \\
5 & 66,500 & 0.506 & 6.32 & 2.19 \\
6 & 76,800 & 0.595 & 6.85 & 2.77 \\
7 & 94,900 & 0.774 & 8.10 & 4.27 \\
8 & 121,000 & 0.940 & 9.50 & 5.76 \\
9 & 147,200 & 1.11 & 11.4 & 7.77 \\
10 & 187,400 & 1.15 & 12.9 & 10.1 \\
11 & 227,000 & 1.19 & 14.9 & 12.5 \\
12 & 274,000 & 1.29 & 16.9 & 15.7 \\
13 & 311,000 & 1.42 & 18.8 & 18.6 \\
\hline
\end{tabular}

$N_{\text {Total }}=\quad 311,000$

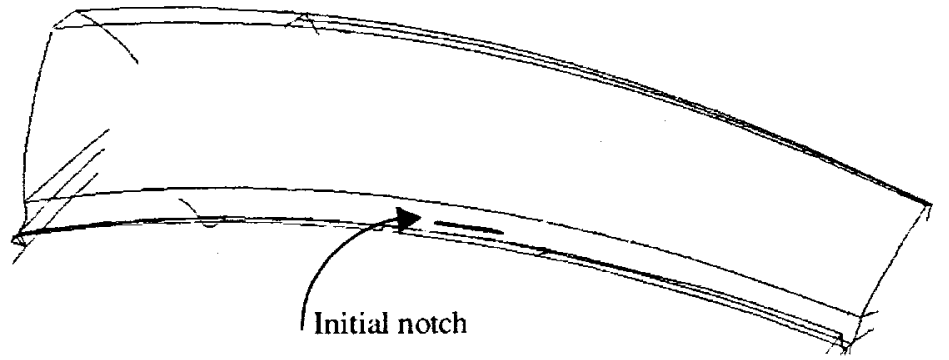

a) Tooth surface

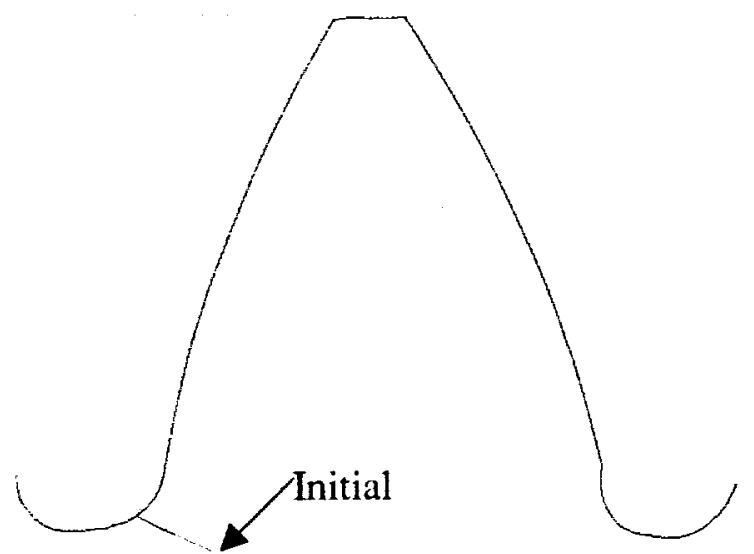

b) Cross section of tooth at midpoint of crack

Figure 5.13: Initial crack; $N=0$ cycles.

\footnotetext{
${ }^{7}$ The approximate location along the tooth length of the initial crack's midpoint is used to measure the crack depth.
} 


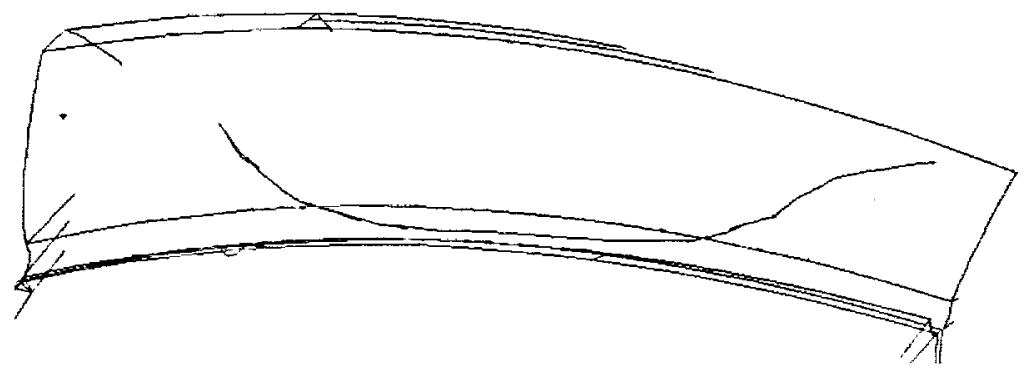

a) Tooth surface

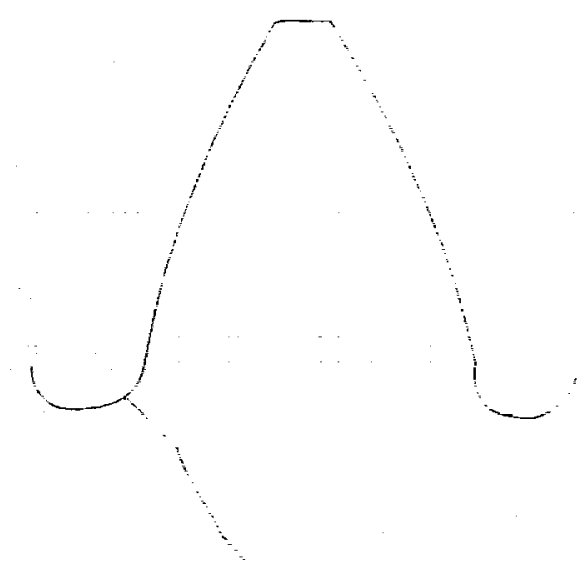

b) Cross section of tooth at midpoint of initial crack

Figure 5.14: Crack prediction after thirteen propagation steps; $N=311,000$ cycles.

In the simulation, the majority of the crack front has progressed almost halfway through the tooth width. A rough estimate of the total life of the gear is made by estimating the tooth width as 0.227 inches at the toe end and using the average growth rate from step thirteen. From these data, approximately 200,000 cycles are necessary for the crack front to progress through the remaining ligament. Therefore, the number of cycles to failure beginning from the initial notch is estimated to be 511,000 . This number could be non-conservative since a constant $K$ is used to calculate the remaining life. On the other hand, the fatigue life prediction does not take into account the cycles leading up to crack initiation. It assumes that the crack begins propagating immediately after the introduction of the notch. It is most likely that in a real gear a number of cycles are attributed to initiation of the crack propagation from the notch.

Figure 5.15 is a plot of the calculated fatigue life. The location along the tooth length of the initial crack's midpoint is used to measure the crack depth, which is plotted against total number of cycles. The crack face area as a function of total number of cycles is also plotted for comparison since the crack depth varies along the crack length. 


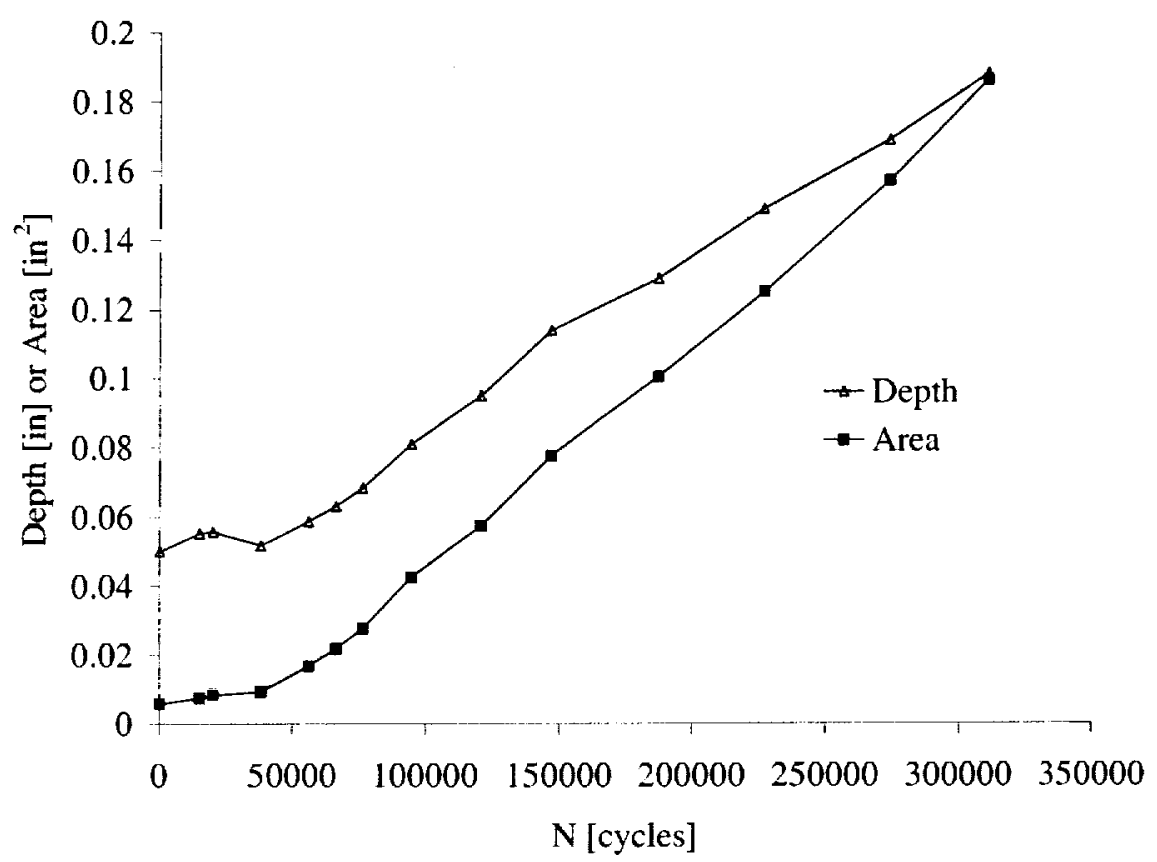

Figure 5.15: Predicted crack depth and crack area versus number of cycles.

The accuracy of the crack trajectory and fatigue life predictions will be evaluated in Chapter 7 by comparing the simulation results to experimental data. The experimental data is obtained from a spiral bevel pinion test that was carried out by NASA/GRC.

\subsection{Chapter Summary}

In this chapter, a boundary element model of a spiral bevel pinion was presented. Different size models were investigated to determine the smallest model which achieved accurate SIF results. An initial crack was introduced into the model and the SIF history along the crack front under the moving load on the pinion tooth was found. It was determined that the loading on the tooth was non-proportional. As a result, a method was developed to propagate the three dimensional crack under the non-proportional load. A crack trajectory prediction in the OH-58 spiral bevel pinion was performed using this method. In Chapter 7 , the simulation results will be compared to experiment results; Chapter 6 presents the experimental data. In addition, in Chapter 7 parameters of the crack propagation method will be investigated to determine the sensitivity of the growth rates and trajectories to variations in the method and crack growth rate model variables. 



\section{CHAPTER SIX: EXPERIMENTAL RESULTS}

\subsection{Introduction}

Experimental results can be used to evaluate the accuracy of the predictions made in Chapter 5. Recordings of crack length and depth as a function of total number of cycles are necessary to confirm the fatigue life prediction. Knowing the crack front shape during propagation would also assist in verifying the predictions.

There is a limited amount of useful experimental data for the $\mathrm{OH}-58$ pinion to validate the numerical results. Test data from an $\mathrm{OH}-58$ spiral bevel pinion are provided by NASA/GRC. The crack growth observations made during the test are limited. As a result, the fracture surfaces of the tested pinion are observed under a scanning electron microscope (SEM). The test data from NASA/GRC and the fractography results are summarized in Section 6.2 and 6.3, respectively. A crack growth scenario during the test is formulated from the SEM observations.

\subsection{Test Results}

A pinion that was tested by NASA/GRC in their spiral bevel gear test fixture under a separate research project is used for comparison/validation. Table 6.1 contains the loading data from the test. A notch was electro-discharge machined (EDM) into the root of a tooth's concave side. The gear was run for six million cycles beginning at $1550 \mathrm{in}-\mathrm{lb}$ torque and progressing up to $4649 \mathrm{in}-\mathrm{lb}$ torque at the end of the six million cycles. The test was not stopped until the completion of the six million cycles; at which time, there was no observable crack growth. As a result, eight more notches of varying sizes were fabricated into individual tooth roots of the pinion. The pinion then ran continuously for an additional 4.9 million cycles at increasing levels of torque detailed in Table 6.1. At the completion of the 4.9 million cycles, five teeth had fractured from the pinion.

Table 6.1: Pinion test data.

\begin{tabular}{|c|c|c|c|}
\hline $\begin{array}{c}\text { Time } \\
{[\mathrm{cycles}]}\end{array}$ & $\begin{array}{c}\text { Speed } \\
{[\mathrm{rpm}]}\end{array}$ & $\begin{array}{c}\text { Torque } \\
{[\text { in-lb] }}\end{array}$ & $\begin{array}{c}\text { \# of EDM } \\
\text { Notches }\end{array}$ \\
\hline 1 million & 6060 & 1550 & 1 \\
\hline 1 million & 6060 & 2324 & 1 \\
\hline 1 million & 6060 & 3099 & 1 \\
\hline 1 million & 6060 & 3874 & 1 \\
\hline 2 million & 6060 & 4649 & 1 \\
\hline 1 million & 4848 & 2479 & 9 \\
\hline 1 million & 6060 & 3099 & 9 \\
\hline 1 million & 6060 & 3874 & 9 \\
\hline 1.9 million & 6060 & 4649 & 9 \\
\hline
\end{tabular}

During the latter 4.9 million cycles, the test was never stopped to observe the crack growth. Therefore, the sequence of events for the tooth fractures and the exact 
number of cycles to failure are unknown. Some of the crack growth may have occurred during the first million cycles at 2479 in-lb torque, or there may have been no crack growth until the last 1.9 million cycles at 4649 in-lb torque. In addition, since no observations of crack growth during the 4.9 million cycles were made, the predicted fatigue life curve (Figure 5.15) can not be validated. The only quantitative information is an upper bound of 4.9 million cycles on the total number of cycles to failure.

a)

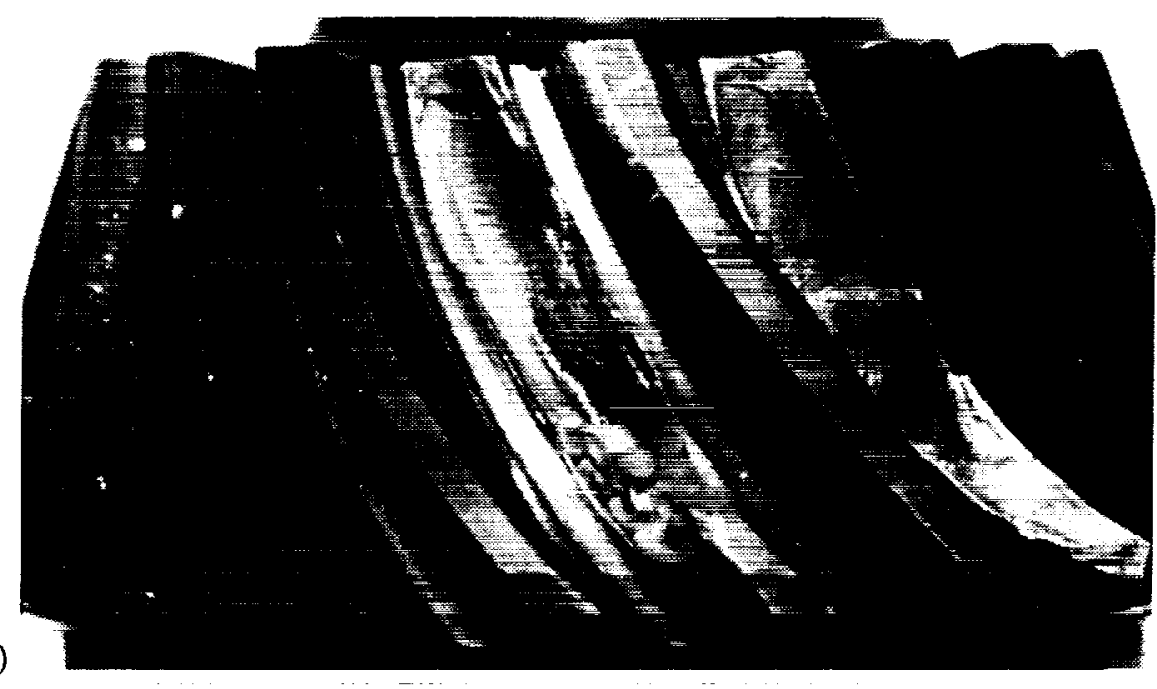

b)

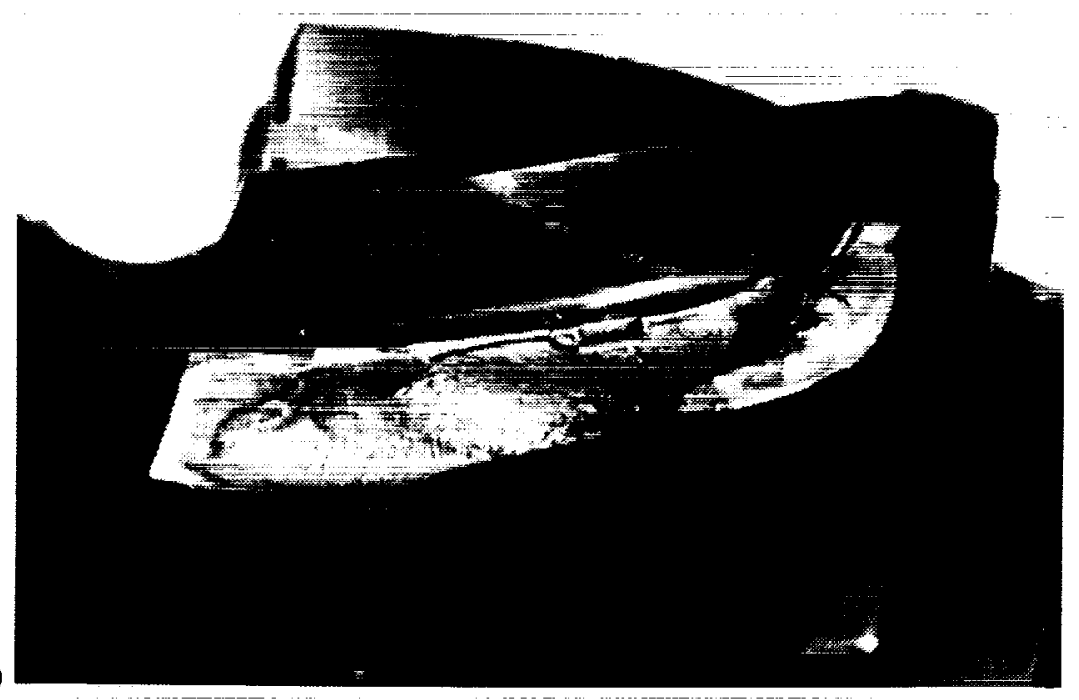

c)

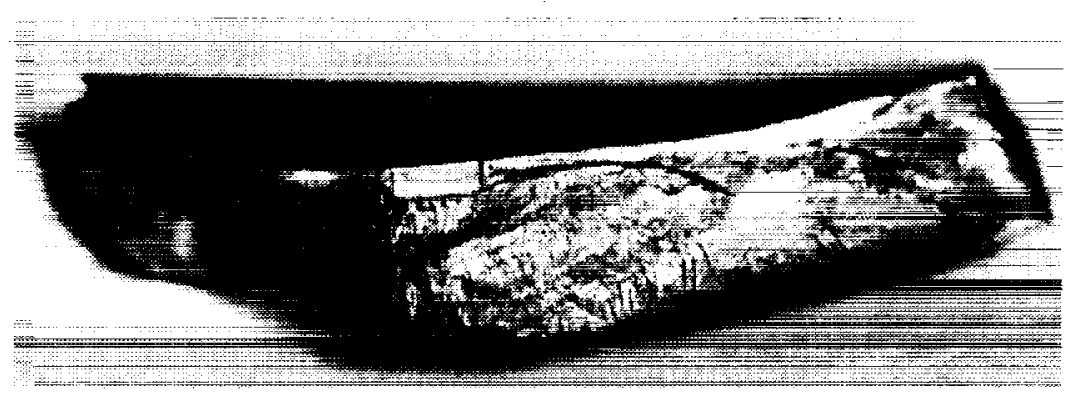

Figure 6.1: Typical tooth failure in tested pinion. 
Qualitative information can be taken from the pinion test to validate the predictions. For example, all five failures were teeth fractures. There was no evidence of a rim type of failure. A macroscopic view of the crack trajectory can be determined from the tested pinion. Figure 6.1 shows photographs of a typical tooth failure in the tested pinion. The rectangular EDM notch on the concave side of the tooth near the root is observable in all of the pictures. The crack trajectory, which is assumed to initiate from the EDM notch, is deeper into the rim in the middle of the tooth length than on the toe end. In addition, a ridge is observed where the crack trajectory turned toward the root of the convex side from the initial path into the rim. All five of the fractured teeth had remaining portions of the tooth left intact at the heel end. Furthermore, four of the notches were small enough such that they do not appear to have grown during the 4.9 million load cycles.

Crack growth rate data and crack front shape information during propagation could both be used to validate the numerical analyses. Since this data was unavailable, the tooth fractures were observed with a SEM. The SEM observations are summarized in the next section.

\subsection{Fractography}

\subsubsection{Overview}

Three teeth that had fractured from the tested $\mathrm{OH}-58$ pinion were examined using a SEM. Tooth \#11 contained a "short" notch $(\sim 4 \mathrm{~mm}$ long $\mathrm{x} \sim 1 \mathrm{~mm}$ deep), and was the initial flaw from which the numerical simulations were based. Tooth \#5 contained a longer and deeper starter notch $(\sim 18 \mathrm{~mm} \mathrm{x} \sim 1.7 \mathrm{~mm}$ deep). Pictures of these teeth's fracture surfaces are contained in this section. The starter notch of the third tooth examined was similar to tooth \#11. No distinguishing features were found on the tooth that were not seen on the other teeth.

There were several goals of the examinations. The primary objectives were to determine how much of the crack growth occurred due to fatigue, where the mechanism of failure might have changed, and, if it did change, to what type of fracture mechanism. Additionally, information on the rate of crack growth and the crack front shape during propagation was desired. All of this information could verify how well the numerical simulations predicted the actual crack evolution.

The sketch in Figure 6.2 shows a typical view of how the crack propagated through a pinion tooth's cross section. The crack initiated from the EDM notch on the side of the tooth with the applied load. This side is the loaded or concave side. The opposite side of the tooth, where the crack ends, is the load free or convex side. The region where the crack deviates from the original path, into the rim, towards the fillet on the convex side will be referred to as the ridge.

Figures 6.3 and 6.4 are of tooth \#5 and \#11, respectively. The fractured teeth are lying on their side with the tooth surface at the bottom of the figure and the ridge at the top. As illustrated in Figure 6.2, the crack growth direction was from the bottom of the photograph toward the ridge at the top of the photograph. The EDM notch is 
the rectangular region along a portion of the tooth length near the bottom right corner and is labeled $\mathrm{A}$ in the figures.

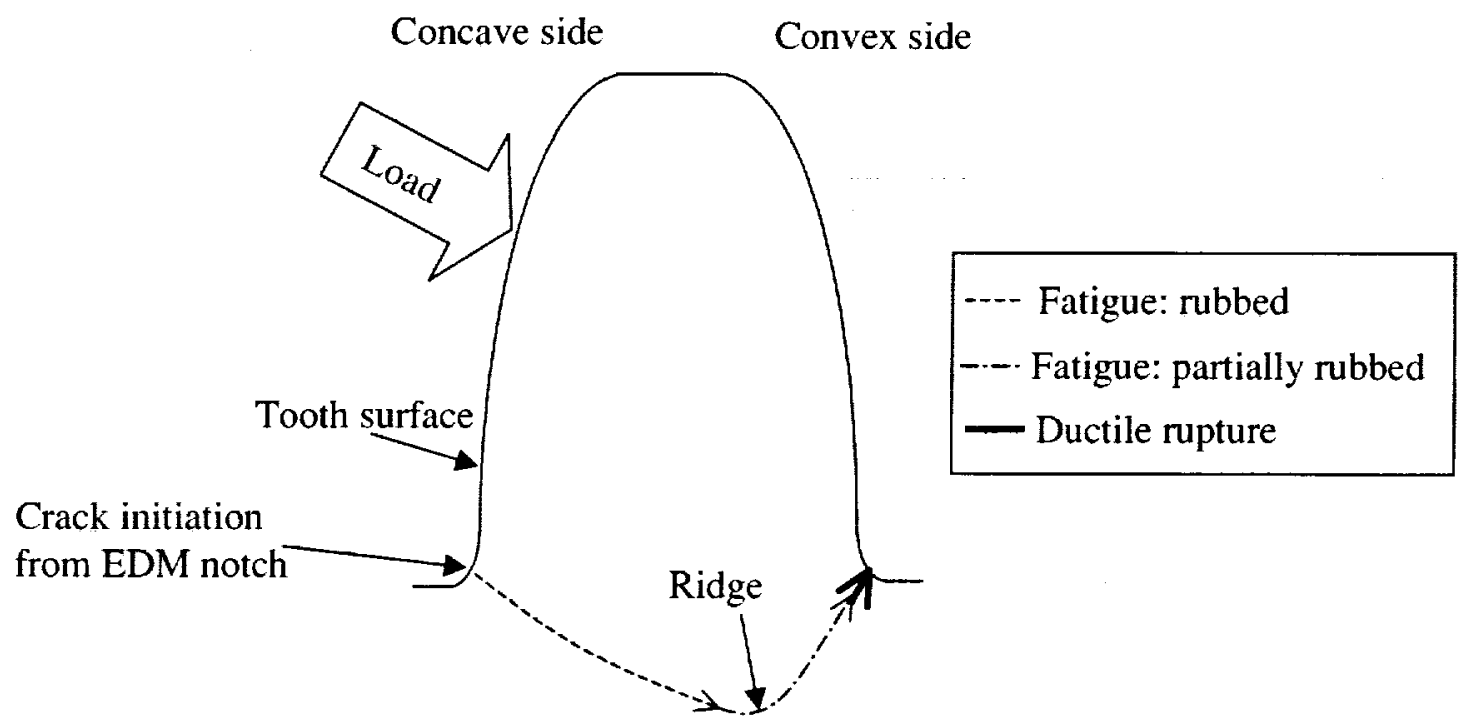

Figure 6.2: Sketch of crack propagation through typical pinion tooth's cross section.

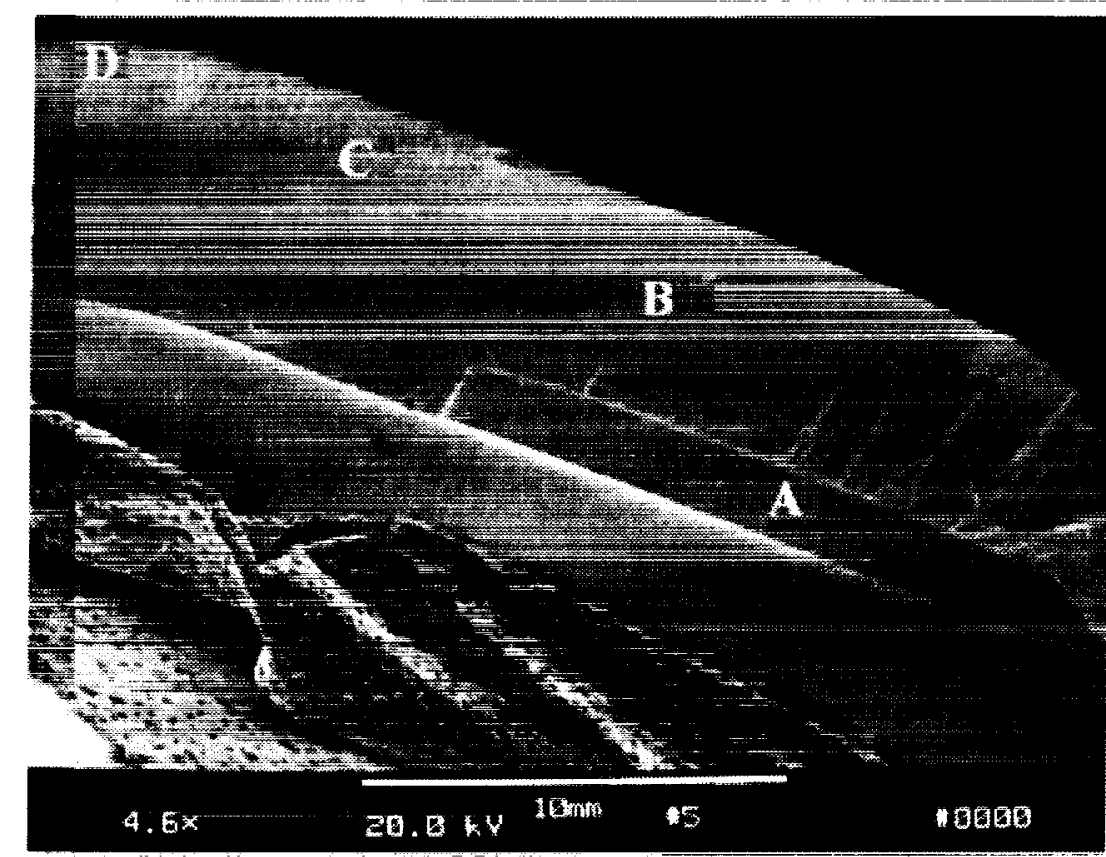

Figure 6.3: Low magnification $(4.6 \times)$ view of tooth $\# 5$ s fracture surface on concave side.

The left side of both Figures 6.3 and 6.4 is the toe end of the tooth length. This is true for all of the concave side pictures in this chapter. On the other hand, the right side of the photographs from the convex side is the toe end of the tooth length. All of 
the photographs of the fracture surfaces designate the tooth number, the level of magnification, and a length scale.

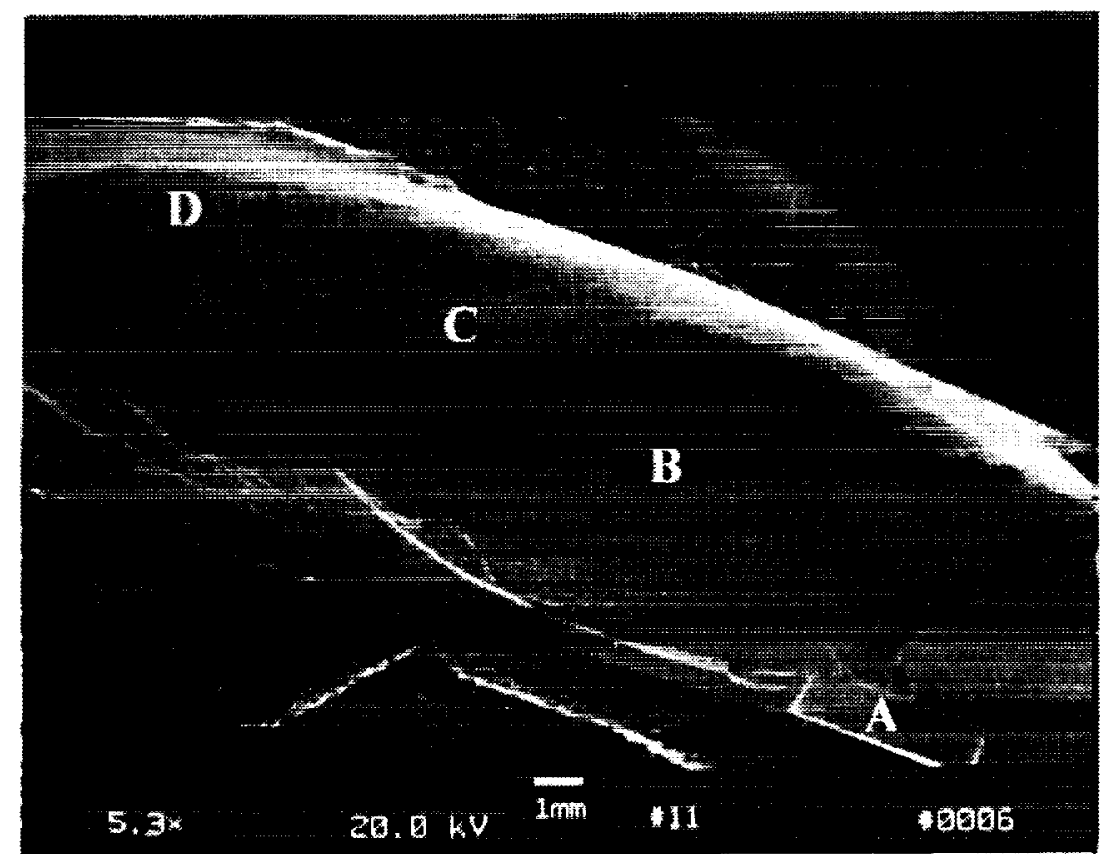

Figure 6.4: Low magnification (5.3×) view of tooth \#11's fracture surface on concave side.

\subsubsection{Results}

The morphologies of teeth \#5 and \#11 are very similar. Lines emanating radially from the starter notch are visible at low magnifications (4.6× in Figure 6.3 and $5.3 \times$ in Figure 6.4). These lines are indicative of fatigue crack growth.

On tooth \#11, some fatigue striations can be seen near the EDM notch. Region $\mathrm{A}$ in Figure 6.5a is the EDM notch. Figure 6.5b is a magnified view of the striated region. ${ }^{8}$ As expected, the striations are roughly parallel to the edge of the EDM notch. The crack growth direction was perpendicular to the striations and, as mentioned above, from the bottom of the figure towards the top. In general, however, most of the surface near the notch was flat with no significant features or texture. This flat surface leads to the conclusion that significant rubbing took place. The rubbing "polished" the surface and removed all features that would have indicated the mode of fracture, e.g. fatigue striations, dimples, etc. The rubbed surface was visible over approximately $80 \%$ of the surface when moving away from the notch toward the ridge of the fracture. Point $\mathrm{B}$ in Figure 6.3 is the approximate location where Figure 6.6 was taken. Figure 6.6 is an example of the typical surface appearance in the rubbed region. ${ }^{9}$ The

\footnotetext{
${ }^{8}$ Figure B.1 in Appendix B also contains magnified views of the striated region.

${ }^{9}$ Figure B.2 in Appendix B is another picture of the typical surface appearance in the rubbed region. Point B in Figure 6.4 is the approximate location where Figure B.2 was taken.
} 
polishing might have resulted from rubbing of the crack faces while the gear was in operation or rubbing against a part of the gearbox after fracturing away from the pinion. The extent and uniformity of the flat, polished surfaces support the former hypothesis.

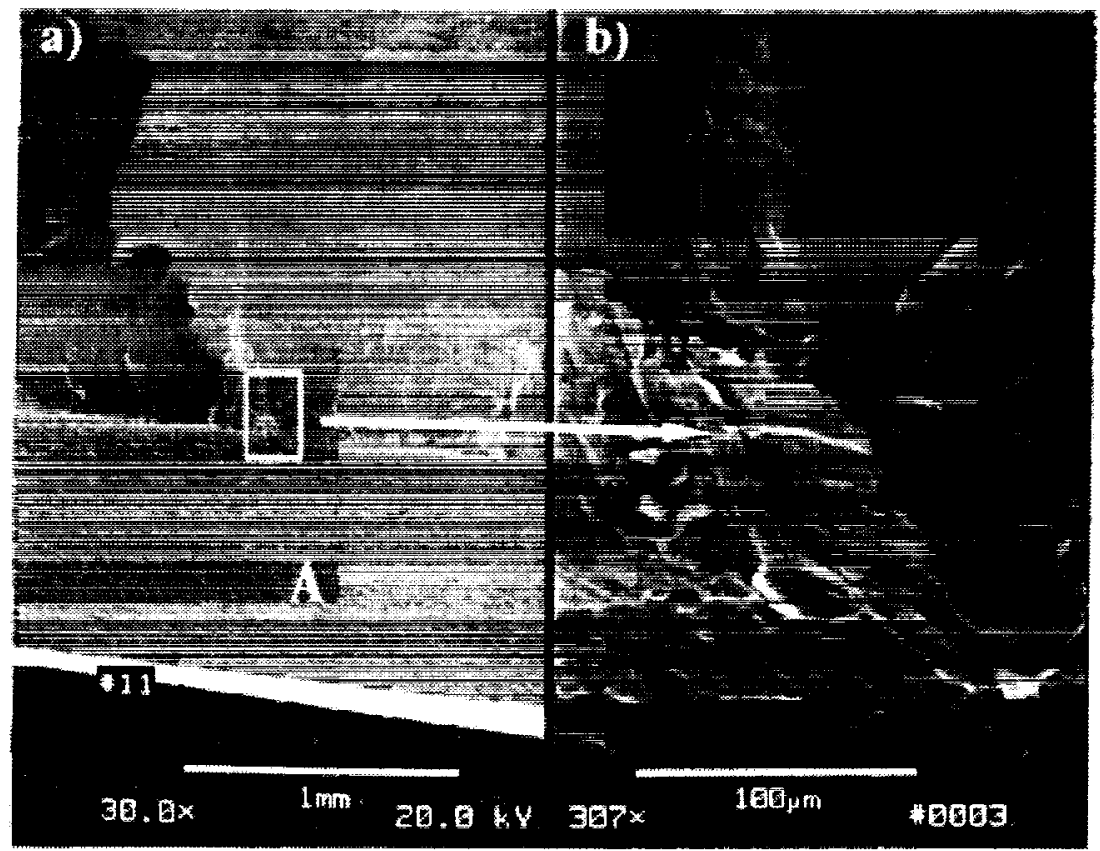

Figure 6.5: Fatigue striations near EDM notch on tooth $\# 11$ at $30 \times(a)$ and at $307 \times(b)$.

A transition from the flat, polished area to one with some texture combined with flattened areas was observed further from the notch near the ridge (point $\mathrm{C}$ in Figures 6.3 and 6.4). In Figures 6.3 and 6.4, this combination, or partially rubbed, type of surface was found along the transition line from the darker region (flat, polished area) to the lighter region of the upper left corner. Recall the light region in both figures is near the toe end of the tooth. Figure 6.7, taken from region $\mathrm{C}$ in Figure 6.3 , shows clearly the features of the partially rubbed surface. The appearance of the raised areas is as if they have been flattened, while the lower lying regions have morphology indicative of fatigue. However, no well developed fatigue striations are observed. 


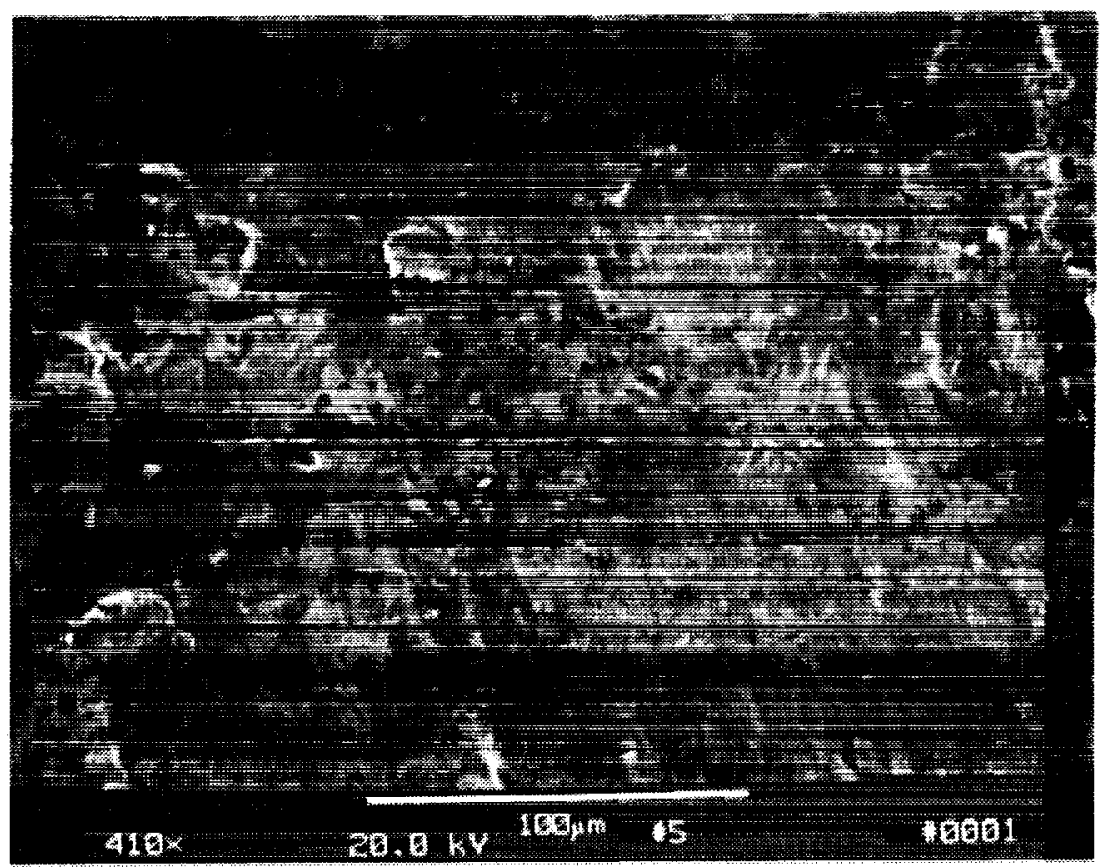

Figure 6.6: Typical picture of flat, polished area on tooth \#5 (410×). Photograph was taken near location B in Figure 6.3.

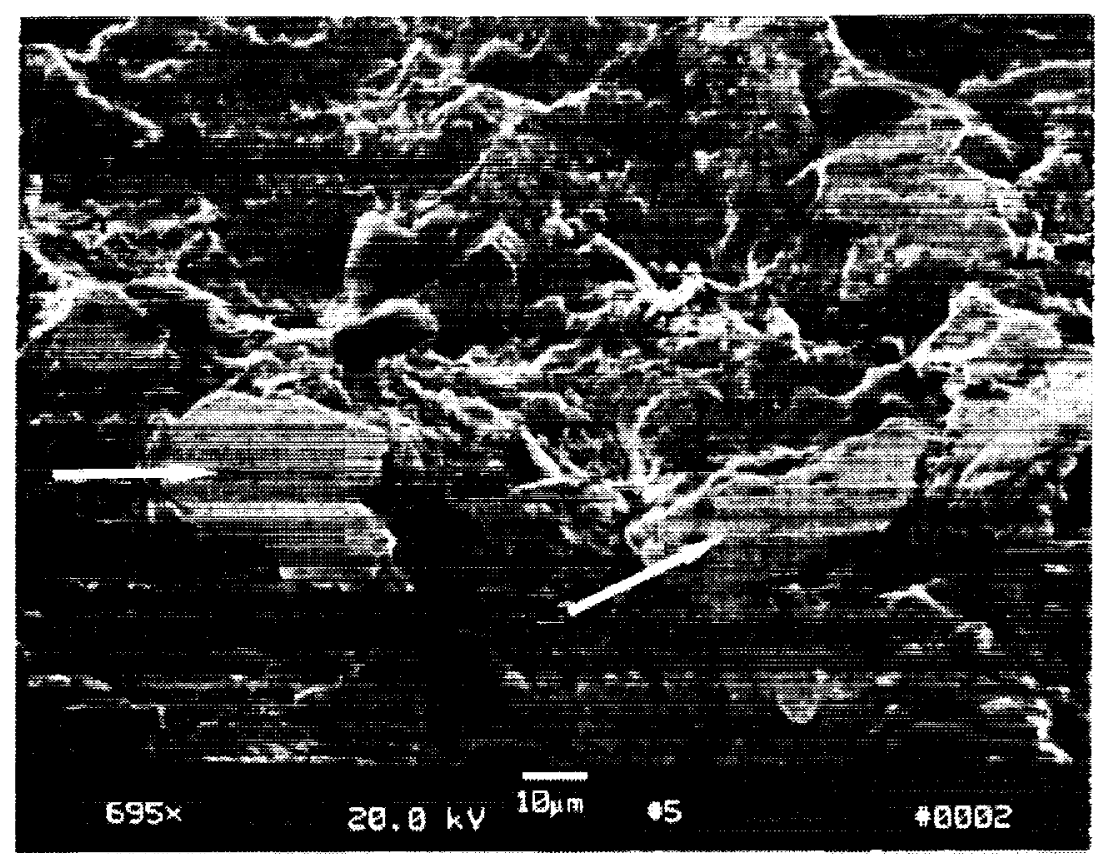

Figure 6.7: Typical picture of partially rubbed surface $(695 \times)$. Photograph was taken from location $C$ in Figure 6.3.

The lighter region in the upper left comers of Figures 6.3 and 6.4 (point D) shows little to no signs of rubbing. The surface also shows no obvious signs of fracture mode, e.g. intergranular fracture, ductile rupture, dimpling. Although no 
striations were present on the surface, the fact that no other obvious signs of failure mode were observed, lead to the conclusion that the crack propagated by fatigue in this region under an applied load range which was inadequate to produce striations. The lack of rubbing also suggests that the fracture surfaces were created in the later stages of crack growth.

The combination type of surface was also found over approximately $90 \%$ of the surface on the load free side of the tooth. Figure 6.8 , of a partially rubbed surface, was taken from location A in Figure 6.9. Region B in Figure 6.9 is the tooth surface on the load free side. Therefore, point $A$ is approximately $0.75 \mathrm{~mm}$ from where the crack ended on the tooth surface. In addition, there are fatigue striations evident in Figure 6.8. Because this figure is from the convex side of the tooth, the crack growth direction was from the top of the figure to the bottom. This combination of evidence leads to the conclusion that the crack continued to grow in fatigue mode along the convex side of the tooth.

A light band can be seen in Region $C$ of Figure 6.9. The darkened region separating region $\mathrm{B}$ and $\mathrm{C}$ is assumed to be oxidation of the fracture surface. Recall that the fatigue striations in Figure 6.8 are from location $\mathrm{A}$. The surface in Region $\mathrm{C}$ shows obvious signs of ductile rupture, Figure 6.10. This observation is encouraging because it demonstrates that the material is capable of failing by ductile fracture, and the areas where this type of fracture occurred should be obvious and visible under the SEM. This result also leads to the conclusion that the primary mode of crack growth on the concave and convex sides of the tooth was fatigue.

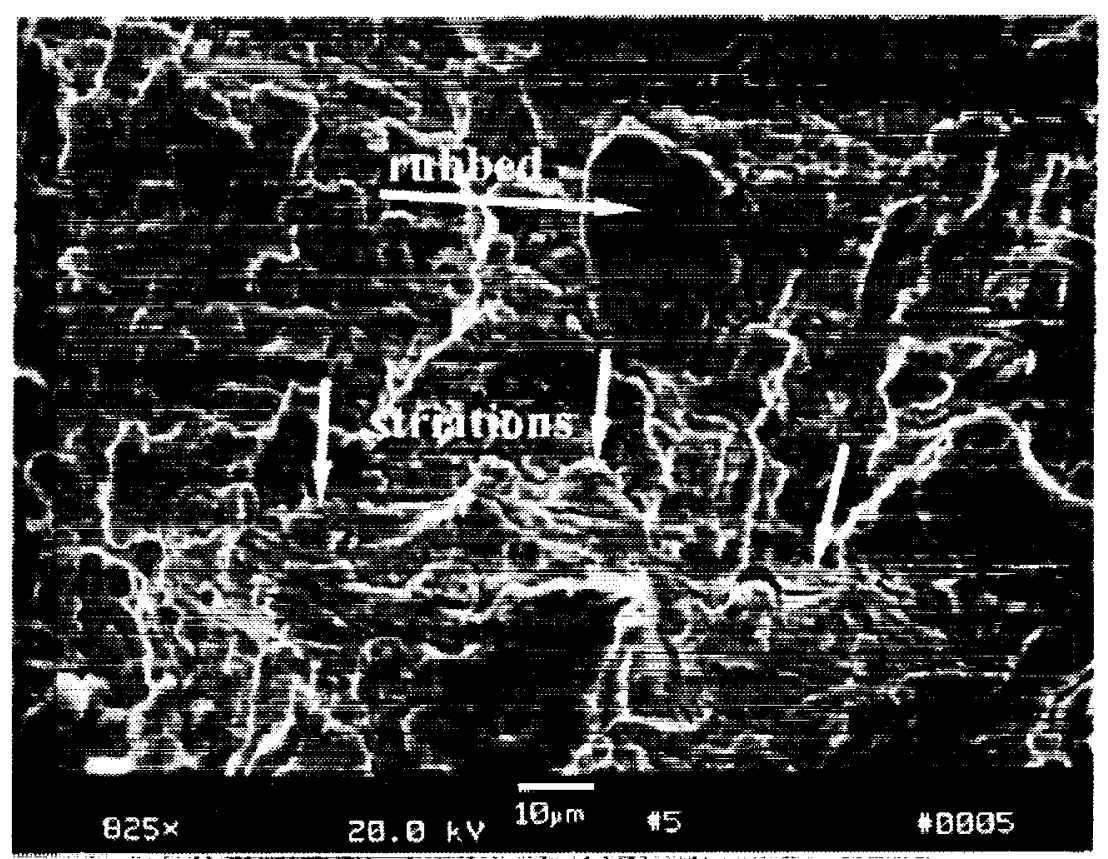

Figure 6.8: Picture of partially rubbed surface with fatigue striations on load free side $(825 \times)$. Photograph was taken at location A in Figure 6.9. 


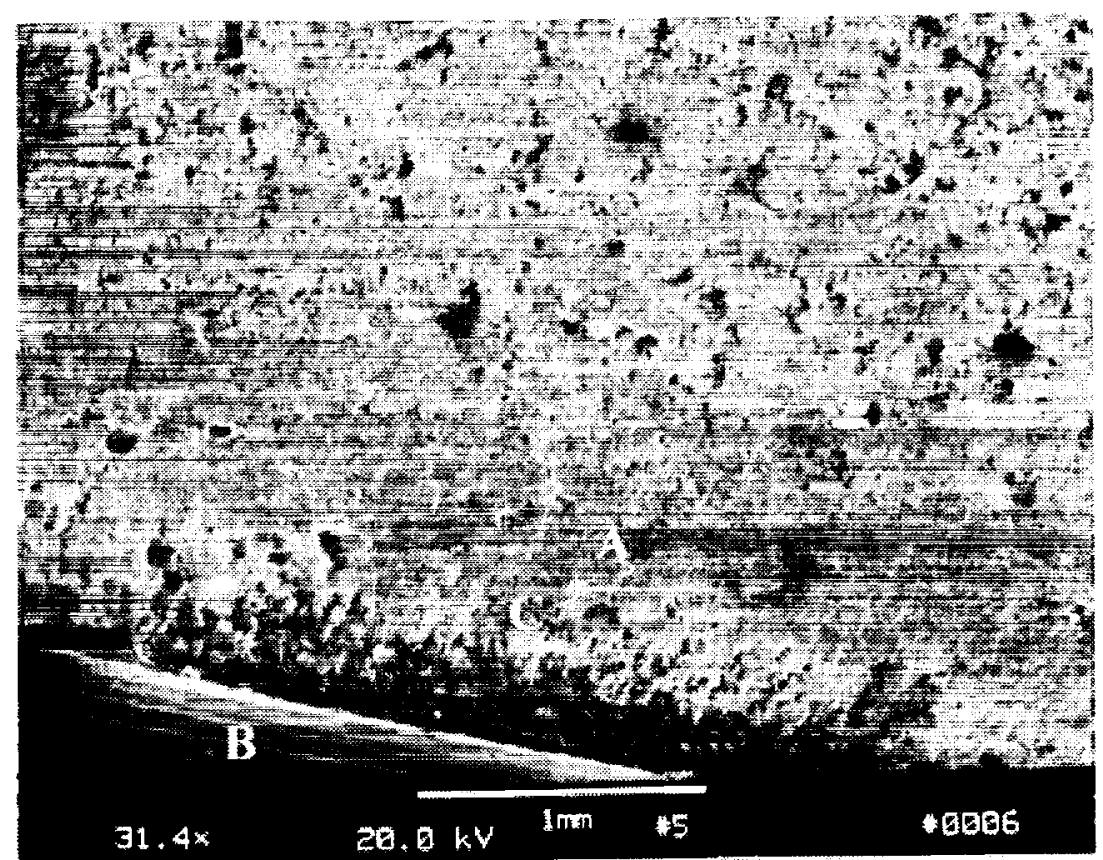

Figure 6.9: Low magnification (31.4×) view of tooth \#5's fracture surface on convex side.

A third tooth (\#9) was also observed with the SEM. All of the features observed on teeth \#5 and \#11, with the exception of the ductile fracture area, were observed on tooth \#9. No additional features could be seen. It is concluded that the observations made of teeth \#5 and \#11 are good representations of the crack patterns on all of the fractured teeth.

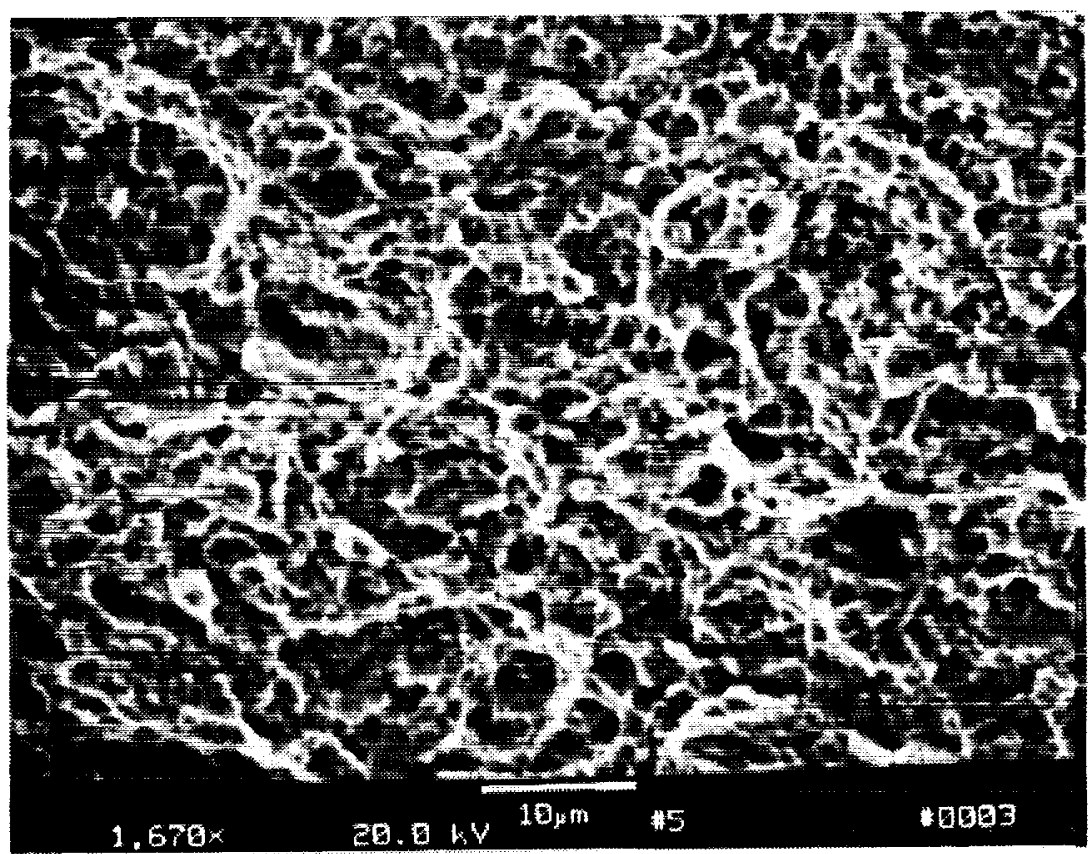

Figure 6.10: Magnified view of ductile rupture at location $C$ in Figure 6.9 (1670×). 
Figure 6.11 summarizes the surface appearance on the loaded and load free sides of the fractured pinion teeth. A scenario of crack growth progression is developed based on these observations. The fatigue crack growth initiates from the EDM notch. The growth continues into the rim and at a larger rate towards the toe than the heel since it is assumed that the rubbed areas are the older surfaces. Once the crack reaches the ridge, the crack continues to grow toward the toe end. Figure 6.12 is a sketch of this scenario on the loaded side. The numbers in the sketch correspond to the progression of the crack front. When the crack reaches the tooth surface at the toe end, the extent of crack growth has dramatically changed the stress distribution in the remaining ligament. Consequently, the crack front turns toward the fillet on the convex side, and progresses by fatigue along the convex side. When the crack front becomes sufficiently close to the root of the convex side, ductile rupture occurs in the remaining ligament. After this, any additional load on the tooth causes the torsional tearing of the ligament on the heel end. Figure 6.2 sketches the crack growth through the tooth width. This sketch is applicable to cross-sections from the toe end to approximately the middle of the tooth length.

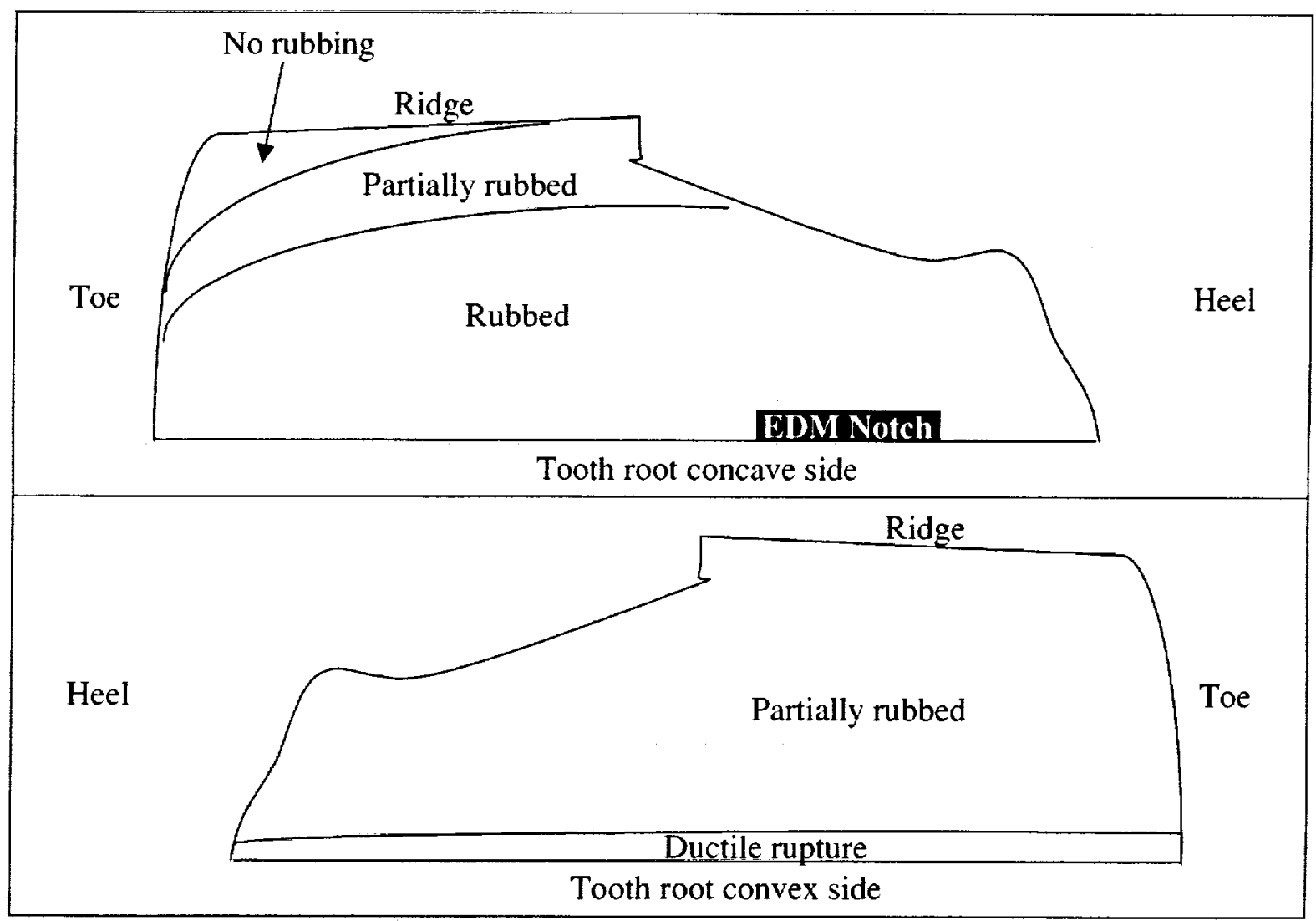

Figure 6.11: Sketch of loaded and load free sides of a pinion tooth's fracture surface appearance along the length. Orientation is consistent with SEM pictures. 


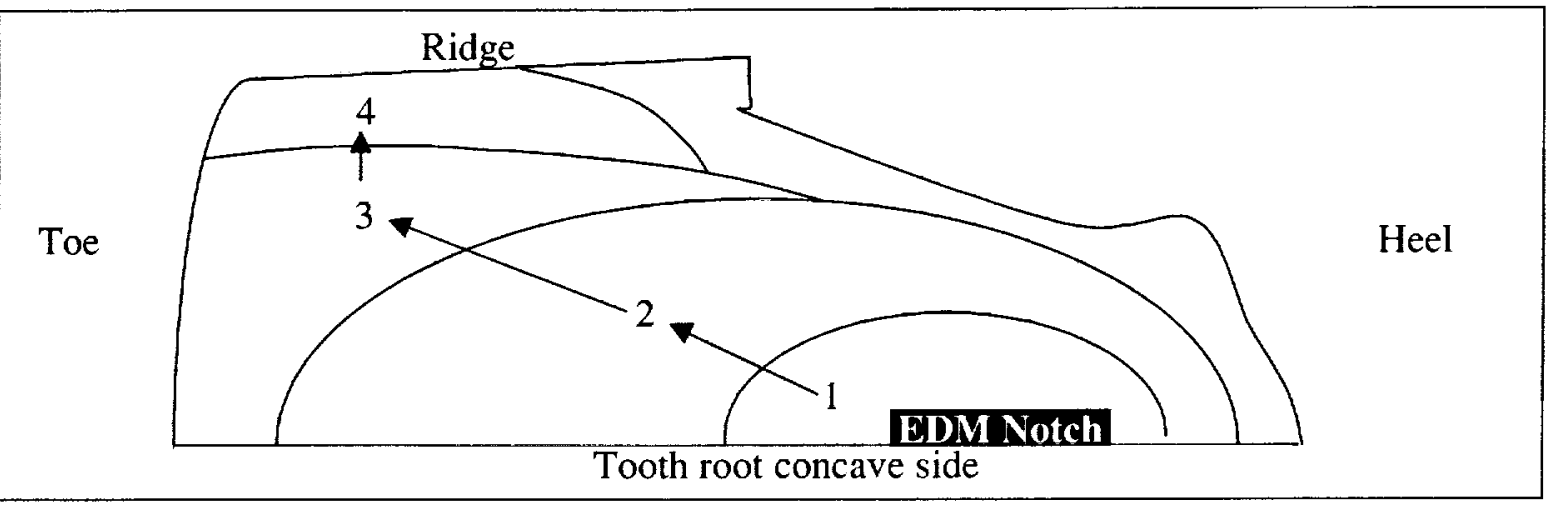

Figure 6.12: Sketch of crack propagation scenario on loaded side devised from fracture surfaces.

\subsection{Chapter Summary}

This chapter was devoted to presenting data from an $\mathrm{OH}-58$ spiral bevel pinion test. The test was conducted by NASA/GRC. EDM notches were introduced into the root of nine of the pinion teeth to serve as starter cracks for fatigue crack growth. Limited observations of the crack growth during the test were made, and, as a result, the fracture surfaces were observed with a SEM.

Overall, the microscopy identified fatigue crack growth regions and regions of ductile rupture successfully. In addition, the crack face morphology showed significant signs of rubbing, which had "polished" the surface. This polishing removed any discernable fracture surface features on the majority of the surfaces. The signs of fatigue on the loaded and load free sides of the fracture surface indicated the majority of the crack growth was attributed to fatigue. At the ridge near the toe end, the surface showed little to no signs of rubbing. This observation suggested that the surface was created in the latter stages of crack growth. It was inferred from the jagged and torn appearance of the fracture surface near the heel that this region was the last remaining ligament of the tooth after rupture occurred in the root of the convex side.

Due to the dearth of well-developed fatigue striations on the fracture surfaces, no observations were made on the crack growth rates. In addition, the large amounts of rubbing removed all indications of crack front shape during propagation. Nevertheless, a scenario of crack propagation was devised. The next chapter compares these test results to the simulations results from Chapter 5. 
$-$ 


\section{CHAPTER SEVEN: DISCUSSION AND SENSITIVITY STUDIES}

\subsection{Introduction}

In Chapter 5, fatigue crack growth in the OH-58 spiral bevel pinion under moving, non-proportional loads was predicted. Chapter 6 presented experimental fatigue crack data from a tested $\mathrm{OH}-58$ spiral bevel pinion. The present chapter compares these two sets of results to evaluate the success of the predictions and investigates the sensitivity of the prediction results to variations in the methods assumptions.

In Section 7.2, the crack growth simulations are compared to the experimental results of a tested pinion; the fatigue lives and crack trajectories are evaluated to determine the accuracy of the prediction method. Sensitivity studies are conducted in Section 7.3 to explore variations in tooth contact position and magnitude and the sensitivity of the crack-closure-based fatigue crack growth rate models to variations in the model parameters.

Crack growth predictions from the moving load analyses (Section 5.5) are compared to crack growth results from analyses that consider only highest point of single tooth contact (HPSTC) loading in Section 7.4. HPSTC loading has been commonly adopted in past research because it is a more simplified approach than the moving load. When using HPSTC loading, existing fatigue crack growth theories can be implemented since there is a single load location and proportional loading. The two loading methods' results are compared to evaluate the need for the moving, nonproportional load method; the least computationally intensive model and method which produces reasonable crack growth results is the most practical for a gear designer.

\subsection{Comparisons of Crack Growth Results}

Figure 7.1 shows the predicted and experimental trajectories on the tooth surface and through the cross section of a pinion tooth. The predicted results are the same as those reported in Section 5.5. The experimental trajectories are approximated from measurements and photographs of the failure surfaces of tooth \#11. As discussed in Chapter 6, the failure associated with tooth \#11 is representative of all the failures in the tested pinion. In addition, the size of the initial flaw in the predictions was taken from the dimensions of the EDM notch in tooth \#11.

A ridge is not observed in the predicted crack path through the cross section because the simulations were stopped prematurely. The simulations were halted because the trajectory along the tooth surface on the toe end varied significantly from the experiment. As a result, it was concluded that the simulations were not completely accurate and further propagation of the crack would lead to no additional insights. An explanation for this discrepancy might be that the simulation did not properly account for changes in the load shape and location as the crack grew. This changing load scenario will be investigated further in Section 7.3.3. 


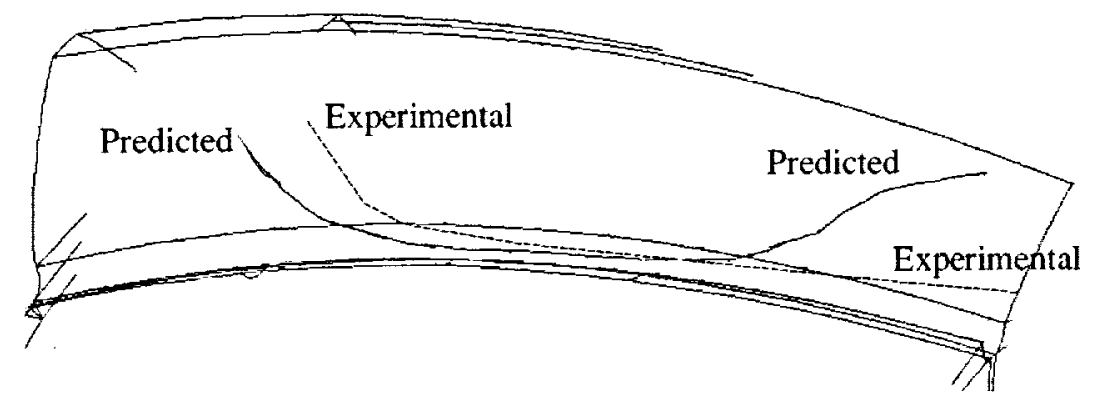

a) Tooth surface

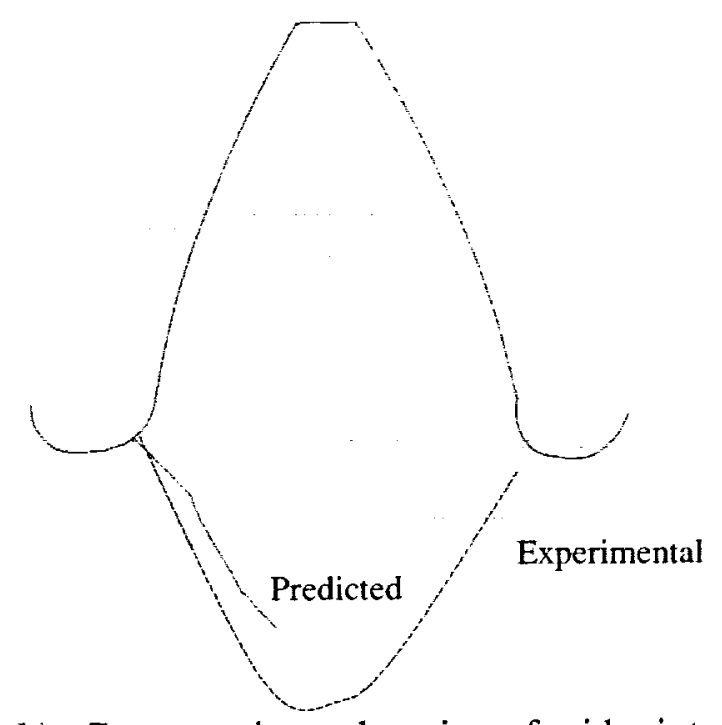

b) Cross section at location of midpoint of initial crack

Figure 7.1: Predicted and experimental crack trajectories.

Qualitative comparisons of the analyses' results to the tested pinion results can be made. The numerical analyses predict tooth failure, which is concordant with the experiment. In addition, in both the fractography study and numerical analyses, the crack propagates more rapidly toward the toe than the heel. In both the test and simulation, a portion of the tooth at the heel end remains intact. Additionally, the simulations predict the crack propagating along a steeper trajectory into the gear rim in the middle of the tooth length than on the toe end of the length. This behavior is also observed in the tested pinion.

The final predicted trajectory through the thickness of the tooth agrees very well with the initial path in the experiment. It is assumed that this path could lead to the formation of a ridge if the simulations were continued. The entire predicted crack trajectory, however, does not completely match the tested pinion. The simulations predict the toe end of the crack turning up the tooth height at a steep angle. This behavior is not seen in the tested pinion. One reason for the discrepancy could be that the loading conditions for the simulations were not identical to the test. The gear was tested at increasing torque levels over the 4.9 million cycles. The simulations, however, were performed under a constant torque level. The increase in torque should affect the tooth contact, which, in turn, will influence the crack trajectory. The influences of the torque level and contact location on crack trajectories are explored in 
Section 7.3.3. The goal is to predict what operating conditions would be necessary to obtain the fracture path observed in the tested pinion.

For the constant torque level of 3120 in-lb, approximately five hundred thousand cycles were predicted to propagate the crack through the tooth thickness. This value is the same order of magnitude as that which occurred in the tested pinion. The number of cycles to produce the tooth fractures in the test is smaller than 4.9 million. The sensitivity of the fatigue life prediction to the values chosen for the model parameters is studied in Section 7.3.1.

Alban's condition number four for "classic tooth-bending fatigue" scenario (Section 2.5) is not captured in the numerical work. The magnitude of the applied loads during the cycle was kept constant during the crack propagation analyses, Figure 7.2a. This type of loading scenario is considered load control and results in the SIFs increasing continuously as the crack grows, Figure $7.2 \mathrm{~b}$. Fatigue crack growth will occur at an increasing rate until the SIFs satisfy a fracture criterion, such as $K_{I}=K_{I C}$. Crack growth simulations under load control will predict a shorter number of cycles to failure than observed. This is because, in reality, when a cracked and uncracked tooth mesh, the cracked tooth will deflect a limited amount before it's adjacent tooth picks up a portion of the load [Alban 1985]. The displacement of the crack faces reaches a maximum and will be roughly equal for every remaining load cycle. As a result, the rate of increase in the SIFs will decrease, and reach roughly a constant maximum every cycle. An idealization of this is shown schematically in Figure 7.2c. Propagating the crack under these conditions is considered displacement control. When the maximum SIF ceases to increase, the fatigue crack growth rate is relatively constant, and the number of cycles to failure increases.

a)

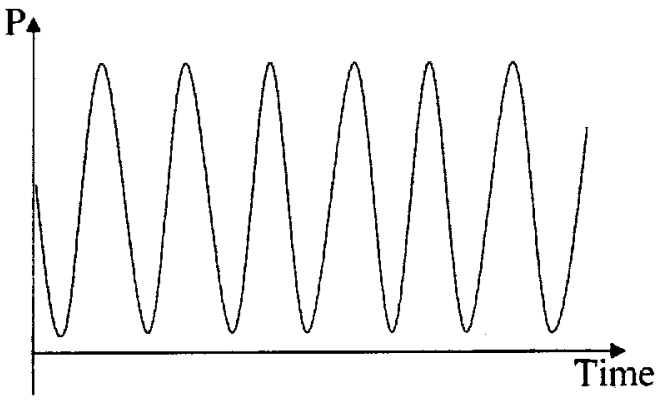

c)

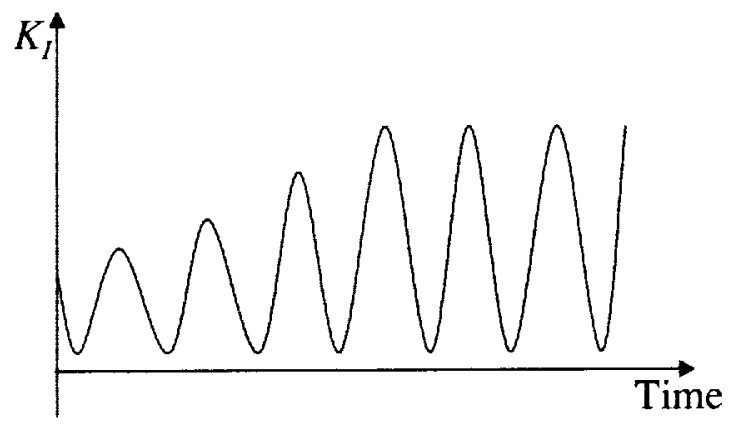

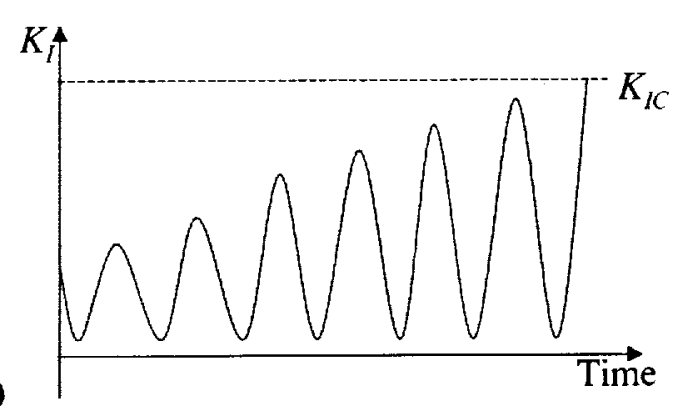

b)

Figure 7.2: Schematic of load cycles: a) Load versus time, b) $K_{I}$ versus time, load control, and c) $K_{I}$ versus time, displacement control. 
To demonstrate that the simulations are capable of predicting the turning that is necessary to predict the ridge in the fractured tooth, a large crack shape is assumed and inserted into the full pinion BEM model. The crack front coordinates are determined by the location of the ridge in a fractured tooth from the experiment. Figure 7.3 is a photograph of the fracture surface with the approximate location of the assumed crack front designated by the dashed line. Based on the SEM observations, the assumed crack has propagated along the root from the initial notch to the toe surface. Figure 7.4 is a picture of the BEM geometry model that illustrates the assumed initial crack trajectory on the tooth surface. Since the correct contact areas for a tooth that is flawed to this large of an extent are unknown, the HPSTC load step from the moving load analyses (load step eleven) is used.

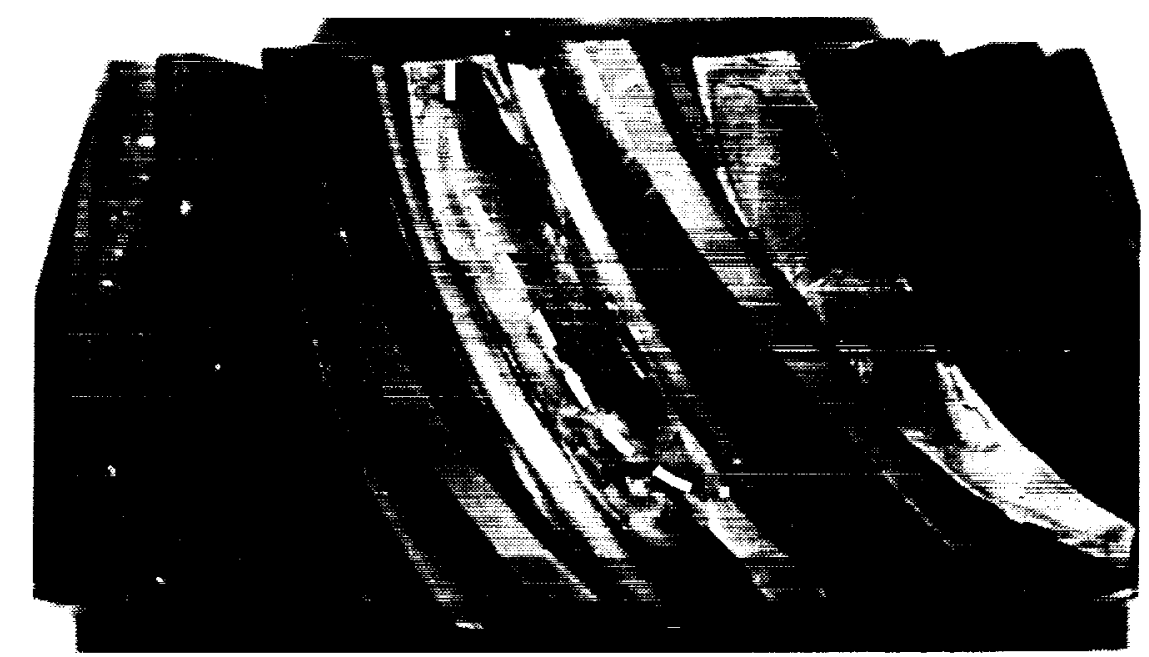

Figure 7.3: Assumed location of crack front (ridge).

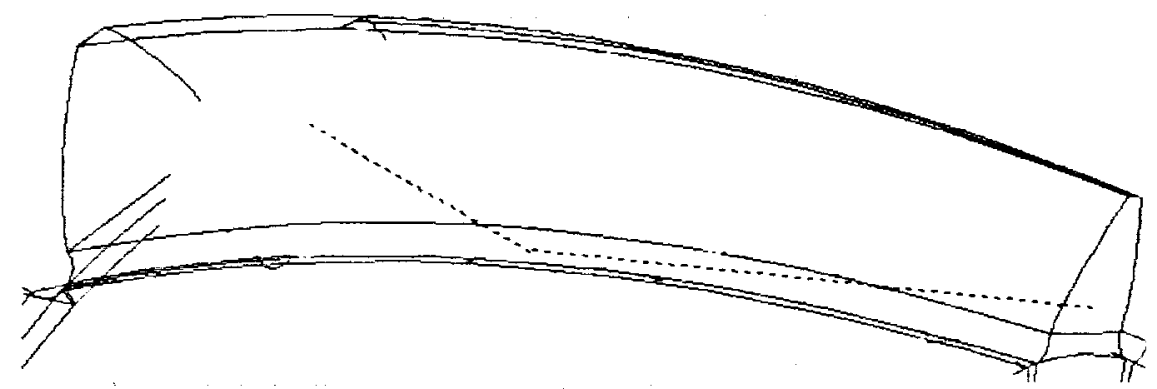

Figure 7.4: Tooth surface showing assumed shape of large crack (dashed line).

A few cycles of crack growth are carried out using the method described in Section 3.2.3. This method assumes mode I dominant fatigue crack growth with static, proportional loading. The cycles of crack growth are necessary to demonstrate the direction the crack front progresses from its assumed location at the beginning of the ridge formation. Figure 7.5 shows the trajectory through the thickness of the tooth at approximately the middle of the tooth length. The initial trajectory into the rim is assumed to be flat, and the curving at the end of the crack length shows the formation of the ridge. This demonstration of the ridge formation based on an assumed crack 
front from the SEM observation supports the crack growth scenario developed in Section 6.3.2.

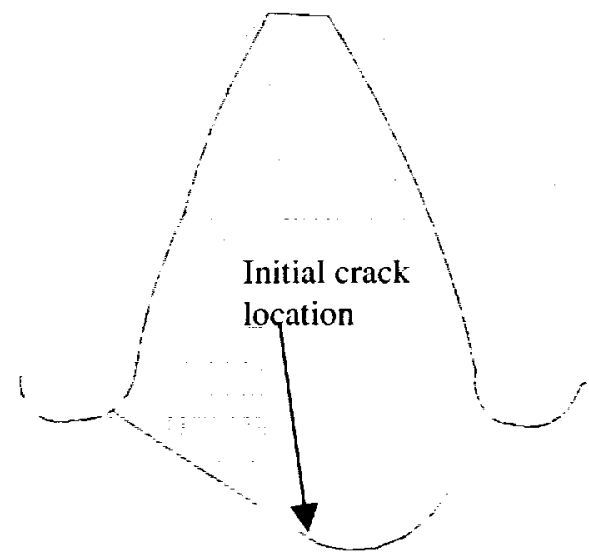

Figure 7.5: Crack trajectory through tooth thickness for assumed large crack.

One could argue that the tested spiral bevel pinion failure did not exactly meet the classical failure conditions described in Section 2.5 since the entire length of the tooth did not fracture from the gear; a portion of the tooth at the heel remained intact. This suggests that the loading might have been biased toward the toe end of the tooth. Numerical analyses with shifted load locations are presented in Section 7.3.3 that give insight into the sensitivity of the crack trajectories to loading location.

The predictions in Section 5.5 did not consider changes in the original contact locations during propagation. The increasing tooth deflections as the crack grows might cause the original contact locations to shift and the distribution of load and the size of the contact ellipses to change. A three dimensional, contact mechanics, and fracture mechanics simulation of the rolling process between two mating gears is necessary to capture the load redistribution effects fully. This type of analysis is not in the scope of this work.

It is impossible to determine the exact amount of rubbing between the crack faces based on the BEM analyses. In Section 6.3.2 it was concluded that the surfaces with greater amounts of rubbing were formed in the earlier stages of crack growth; since these surfaces were older, the features of the surfaces had more time to rub away. The kinematics of the geometry and loading is another explanation for the varying amounts of rubbing observed on the fracture surfaces. Rather than attribute the varying degrees of rubbing to time, it could be attributed to the magnitude of contact between the fracture surfaces. The loading might deflect the tooth in a manner that does not allow the ridge's fracture surfaces to rub, but does create large contact forces between the crack faces near the notch. A three dimensional analysis modeling contact between the crack faces with accurate loading conditions on the tooth surface is necessary to determine the true cause of the rubbing.

\subsection{Sensitivity Studies}

These studies are performed to gain insight into the sensitivity of predicted crack growth rates and predicted crack trajectories to growth rate model assumptions, load magnitude, and load location. They are also conducted to investigate possible 
causes for the discrepancies between the predictions and the experimental results. The fatigue crack growth rate model parameters, Section 7.3.1, crack closure model parameters, Section 7.3.2, and the contact position and magnitude, Section 7.3.3, are researched further.

\subsubsection{Fatigue Crack Growth Rate Model Parameters}

Limited fatigue crack growth rate data is available in the literature for AISI 9310 steel. The predictions in Section 5.5 used values for the crack growth rate model parameters, $n$ and $C$, taken from a curve fit to the intrinsic fatigue crack growth rate data (no closure) for AISI 9310 tested in $250^{\circ}$ oil. These values were 3.36 and $6.19 \mathrm{e}-20(\mathrm{in} / \mathrm{cycle}) /\left(\mathrm{psi}^{*} \mathrm{in}^{0.5}\right)^{n}$, respectively. The range of values for $n$ and $C$ from the literature is reported in Table 7.1. Au et al.'s data are not from intrinsic fatigue crack growth rate curves. Their data are from fatigue crack growth tests with $R=0.05$. The other three sets of model parameters have been normalized to an intrinsic fatigue crack growth rate curve.

Table 7.1: Fatigue crack growth rate model parameters.

\begin{tabular}{|c|c|c|c|c|c|}
\hline Source & $n$ & $\begin{array}{c}C \\
{\left[(\mathrm{in} / \mathrm{cycle}) /\left(\mathrm{psi}^{*} \mathrm{in}^{05}\right)\right]^{\prime \prime}}\end{array}$ & $\begin{array}{c}d a / d N^{10} \\
{[\text { in/cycle] }}\end{array}$ & $\begin{array}{l}\text { Cycles } \\
\text { /inch }\end{array}$ & $\begin{array}{c}\text { Fatigue } \\
\text { Life }^{1}\end{array}$ \\
\hline $\begin{array}{l}\text { Forman et al. } \\
\quad[1984]\end{array}$ & 1.63 & $1.08 \mathrm{e}-13$ & $9.30 e-6$ & 107,527 & 675,838 \\
\hline $\begin{array}{l}\text { Au et al. } \\
{[1981]^{11}}\end{array}$ & 2.56 & $2.72 e-17$ & $2.03 e-6$ & 492,611 & $3,096,201$ \\
\hline $\begin{array}{c}\text { Air test } \\
\text { [Proprietary 1998] }\end{array}$ & 3.63 & $5.49 \mathrm{e}-21$ & $1.54 \mathrm{e}-5$ & 64,935 & 408,145 \\
\hline $\begin{array}{c}\text { Oil test } \\
\text { [Proprietary 1998] }\end{array}$ & 3.36 & $6.19 \mathrm{e}-20$ & $1.23 \mathrm{e}-5$ & 81,301 & 511,000 \\
\hline
\end{tabular}

The fatigue life estimates using each set of parameters in Table 7.1 assume that the number of cycles that each source would predict for the gear's fatigue life is roughly proportional to the ratio of the oil test's cycles/inch to each set's cycles/inch. Forman et al., Au et al., and the air test data each predict a fatigue life of 675,838 cycles ( $32 \%$ increase), $3,096,201$ cycles ( $506 \%$ increase), and 408,135 cycles ( $20 \%$ decrease), respectively.

Au et al.'s combination of $n$ and $C$ predicts the smallest growth rate and therefore the longest fatigue life. The benefits and conservatism of considering crack closure in the predictions is demonstrated by comparing the predictions using the intrinsic parameters to the predictions with Au et al.'s parameters. Figure 7.6 contains the fatigue crack growth rate curves for the various sets of $n$ and $C$. The curves are generated from the data in Table 7.1.

${ }^{10}$ Calculations are based on an assumed value for $\Delta K=18,000 \mathrm{psi}^{*}{ }_{\mathrm{in}}{ }^{0.5}$.

"This data was taken from a fit to fatigue crack growth rate data for non-carburized AISI 9310 tested in wet air, at a loading frequency of $1.0 \mathrm{~Hz}$, and $R=0.05$. The parameters are not from intrinsic fatigue crack growth rate data. It is should be noted that when parameters from the air test at $R=0.05$ are used the calculated growth rate is $2.02 \mathrm{e}-6 \mathrm{in} /$ cycle. 


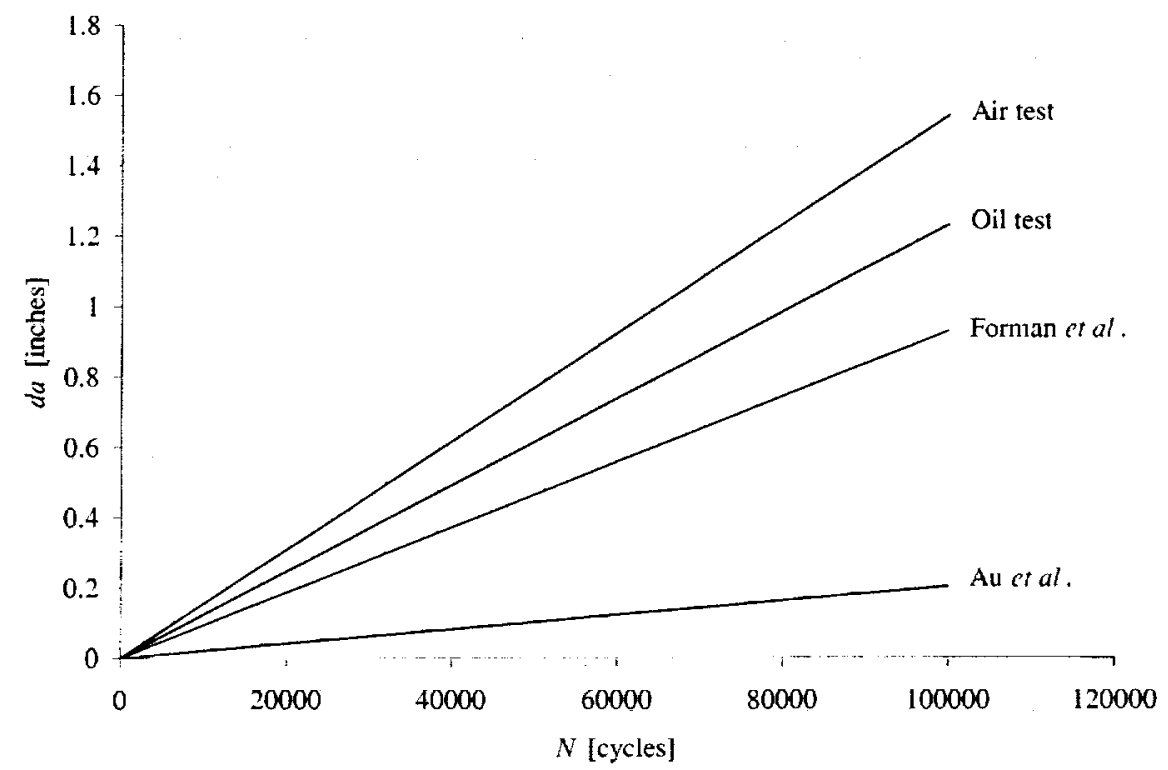

Figure 7.6: Fatigue crack growth rate curves for the sets of model parameters in Table 7.1.

An exact evaluation of which set of material constants is most accurate is nearly impossible since many parameters of the pinion test are unknown. The calculations presented in this section demonstrate a trend that the fatigue life calculations will be more accurate when material constants from intrinsic fatigue crack growth rate curves are used.

\subsubsection{Crack Closure Model Parameters}

For the simulation results presented in Section 5.5, values for the crack closure model parameters, $\kappa$ and $\beta$, were assumed in order to calculate the fatigue crack growth rates. This section investigates the validity and sensitivity of the results to the assumed values.

\section{Crack Growth Rate Sensitivity to $\kappa$}

$\kappa$ incorporates three dimensional effects into the crack growth rate calculations. Newman specifies that $\kappa$ varies between one and three for plane stress to plane strain, respectively. For the predictions reported in Section $5.5, \kappa$ was equal to three. However, for extremely shallow cracks or portions of the crack front near the free surface, a value of $\kappa$ equal to one might represent the crack conditions more accurately.

One method to evaluate the crack tip conditions is to compare the size of the crack tip plastic zone to the crack's geometry. The plastic zone is larger in plane stress than in plane strain. An approximation of the plastic zone size, $r_{p}$, is

$$
r_{p} \approx \frac{1}{3 \pi}\left(\frac{K_{I}}{\sigma_{y}}\right)^{2}
$$


Using load step eleven's SIF results from the initial crack (Figure 5.8), which are the largest mode I SIFs during the load cycle, the plastic zone size along the crack front ranges from $1.44 \times 10^{-4}$ inches to $1.07 \times 10^{-3}$ inches. These dimensions are only $0.29 \%$ and $2.14 \%$, respectively, of the initial crack depth. It is concluded, therefore, that the plane strain assumption along the entire crack front most accurately represents the conditions in the real gear.

\section{Crack Growth Rate Sensitivity to $\beta$}

$S_{\max }$, the far field applied stress, is a function of $\beta, c$ (half of the crack length), and $K_{l} . \beta$ is a dimensionless quantity that considers geometry effects when relating $K_{I}$ to the applied stress. Values of $\beta$ from handbook solutions can vary from one half to two [Murakami 1987]. Since the gear geometry is complex and unlike any handbook solution, a value of $\beta=1$ was selected. An alternate approach could have been to use a known $\beta$ factor for a similar, simplified geometry. This alternate approach will now be investigated and growth rates between the two methods will be compared.

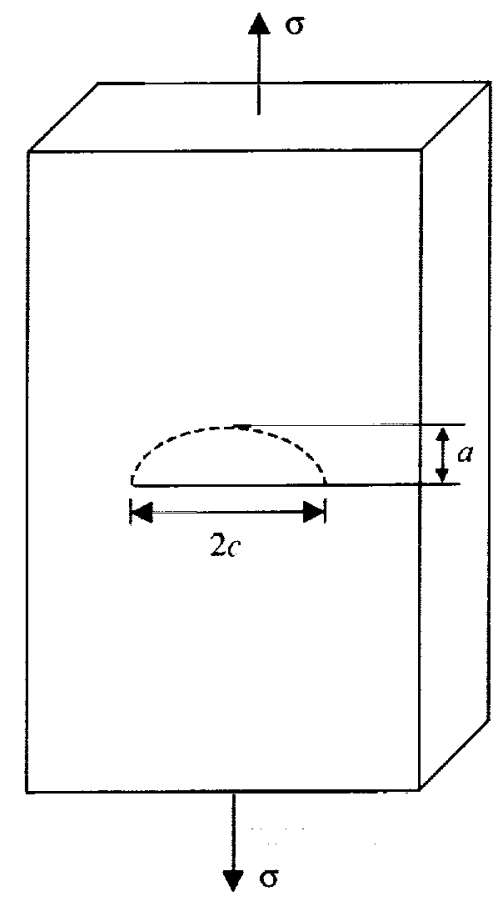

Figure 7.7: Finite thickness plate with a semi-elliptical surface crack subjected to mode I uniform stress.

The initial crack in the gear is approximated by a finite thickness plate with a semi-elliptical surface flaw subjected to mode I uniform tensile stress, $\sigma$, Figure 7.7. The magnitude of $K_{I}$ varies along the crack front. $K_{I \max }$ and $K_{I \min }$ occur at the surface and midpoint of the crack front, respectively. They are given by Broek [1986] as:

$$
K_{\text {Imax }}=\frac{1.12}{\phi} \sigma \sqrt{\pi a} \text { and } K_{\text {Imin }}=\frac{1.12}{\phi} \sqrt{\frac{a}{c}} \sigma \sqrt{\pi a}
$$




$$
\text { where } \phi \approx \frac{3 \pi}{8}+\frac{\pi}{8}\left(\frac{a}{c}\right)^{2}
$$

From Equation (7.2), the maximum and minimum expressions for $\beta$ are

$$
\beta_{\text {max }}=\frac{1.12}{\phi} \text { and } \beta_{\text {min }}=\frac{1.12}{\phi} \sqrt{\frac{a}{c}}
$$

Based on the initial crack geometry, $\beta_{\max }$ and $\beta_{\min }$ are 0.783 and 0.701 , respectively. $K_{o p}$ and $d a$ are recalculated using these values for $\beta$, the SIFs from the initial notch analyses (Figure 5.8), and the same model parameters as were used in Section 5.5.

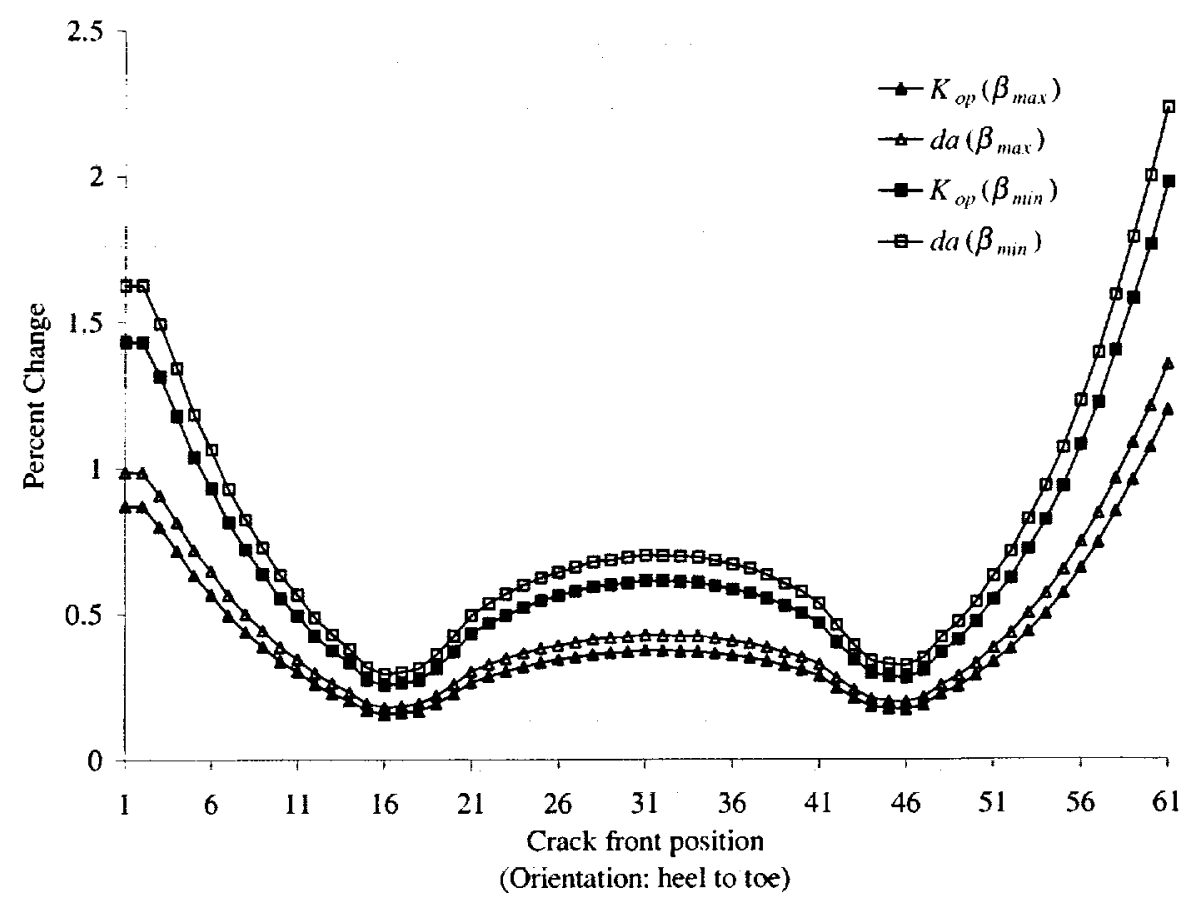

Figure 7.8: Change in $K_{o p}$ and $d a$ as functions of $\beta_{\max }$ and $\beta_{\min }$ with respect to original calculations with $\beta=0$.

Figure 7.8 shows the percent change of $K_{o p}$ and $d a$ with respect to the original calculations. The data show that the largest percent difference is $2.2 \%$. As the crack grows the ratio of $a$ to $c$ will become smaller since the tooth length is longer than the tooth width. This will increase $\phi$ and, therefore, increase $\beta_{\text {max }}$ and decrease $\beta_{\text {min }}$. However, as the crack grows, this closed form solution for $K_{l}$ in the gear is no longer valid since the crack's geometry changes dramatically. Therefore, no further conclusions can be stated on the effect of $\beta$ on crack growth calculations.

\subsubsection{Loading Assumptions}

The intent of this study is to determine how the crack trajectory changes under different contact conditions. One motivation for this is that the simulation and experiment's crack trajectories on the toe end do not match. The tested pinion's crack mouth remained relatively flat along the root until it reached the end of the tooth 
length at the toe (Figure 6.1b). The trajectory in the simulation turned, out of the root, up the tooth height and eventually reached the top land (Figure 5.14a). It is hypothesized that the differences may be attributed to inconsistencies between the contact conditions (loading conditions).

The inconsistencies could result from misalignment during the test or inaccurate representation in the simulation of the actual contact areas in the test. Glodez et al.'s [1998] experimental work with spur gears supports this hypothesis. They considered two load cases: i) loading along the entire length of the tooth, and ii) loading along one half of the length. With load case ii) the crack in the unloaded portion of the tooth length turned out of the root and grew up the tooth height. On the other hand, the crack in load case i) remained flat along the entire length of the tooth root. The goal of the remainder of this section is to investigate whether shifted loads have the same influence on crack trajectories in spiral bevel gears as Glodez et al. showed in spur gears.

\section{Load Location}

Two shifted load scenarios are investigated. For both scenarios, the cracked BEM model from propagation step number five is analyzed under the shifted contact. This model was chosen because the crack trajectory began turning sharply from this step onward in the preliminary analyses. The contact areas are shifted approximately \pm 0.3 inches along the tooth length.

The crack trajectory for the shifted contact areas is calculated using the nonproportional load method described in Section 5.4.2. The load cycle is approximated by the discrete load steps one, five, and eleven. Table 7.2 sketches the predicted trajectories on the tooth surface for the original and two shifted analyses. The mode I and II SIFs from the shifted loading scenarios are given in Appendix C.

When the contact is central, the crack turns up the tooth height on both ends. The trajectory "wraps around" the contact location. However, when the contact is shifted toward the heel (toe), the tendency for the crack to kink up on the heel end (toe end) is suppressed. This is most clearly seen when comparing the central and toe contact location trajectories. This result is consistent with Glodez et al.'s observations. As a result, it is assumed that, if the fatigue crack growth simulations were carried out further with the shifted contact locations, a flatter trajectory that maintains a path very near the root under the contact location will result.

The discrepancy of the toe end trajectory between the test and simulation is explained by the fact that, in the test, the contact was closer to the toe end. The shifted contact could have resulted from increasing deflections of the tooth. As the crack grew, the tooth's stiffness decreased, and the load could have been redistributed along the tooth length. The subtleties of the redistribution and its effect on crack trajectories can only be modeled accurately with a three dimensional contact analysis between the mating gears in conjunction with a fracture mechanics simulation. 
Table 7.2: Crack trajectories from contact locations shifted along tooth length.

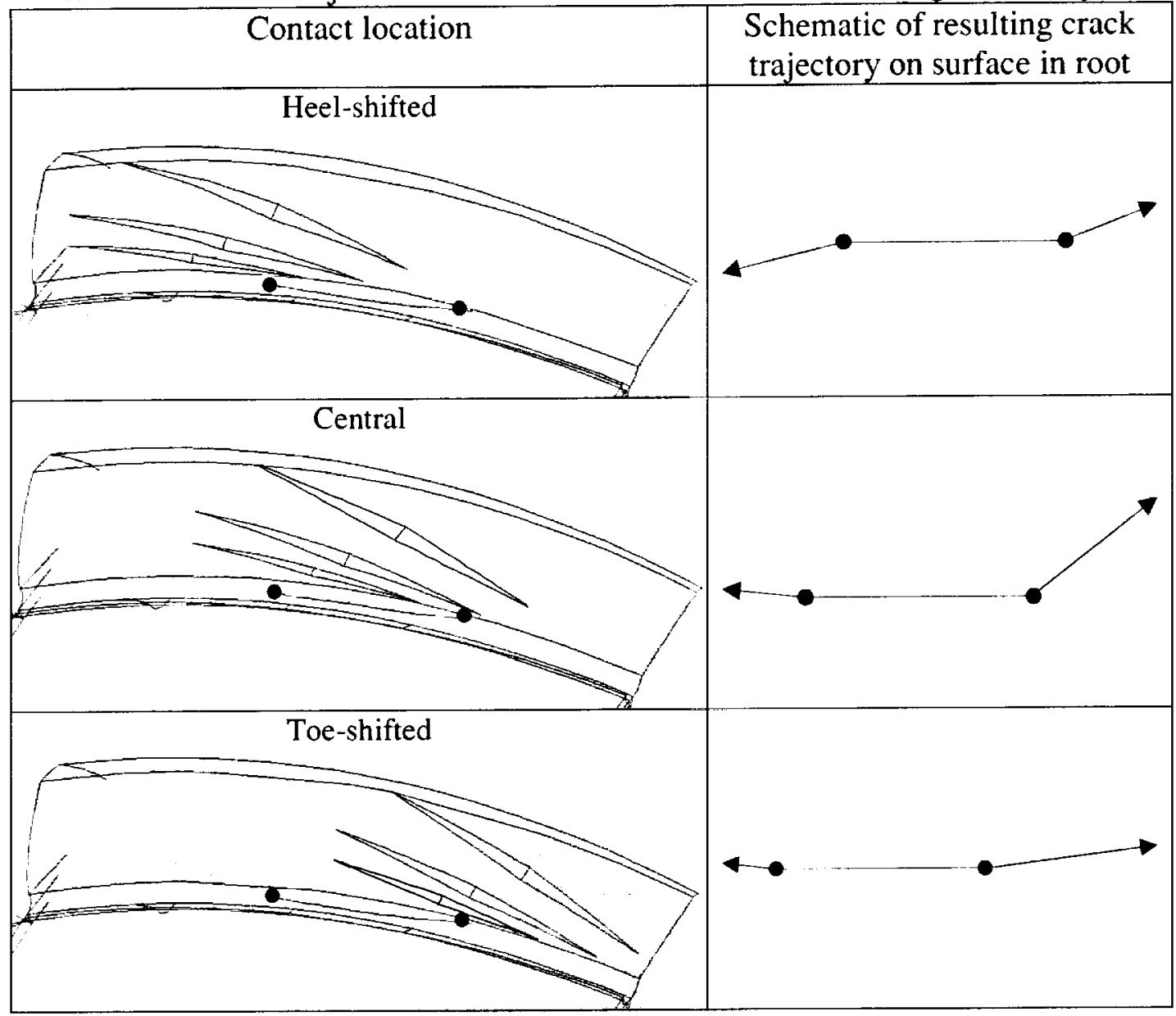

\section{Load Magnitude}

The tested spiral bevel pinion was run at varying levels of input torque detailed in Table 6.1. However, the simulation results reported in Section 5.5 assumed contact areas and load magnitudes produced by $3120 \mathrm{in}-\mathrm{lb}$ torque (100\% design load). The goal of the current study is to identify the influences of the increased torque levels on crack trajectories.

The SIF distributions and trajectories under larger torque levels of 3874 in-lb and 4649 in-lb (125\% and 150\% design load, respectively) are explored. From Hertzian contact theory, it is known that the lengths of the contact ellipses' axes are proportional to the cube root of the applied load (Equation (2.1)). Consequently, the lengths of the major and minor axes increase by $7.72 \%$ (125\% design load torque) and $14.47 \%$ ( $150 \%$ design load torque) under the larger loads. It is assumed that the mean contact points (center of the ellipses) are the same as the points defined for the $100 \%$ design load. Similar to the shifted load analyses, the crack from propagation step five in the moving load simulations (Section 5.5) is selected to analyze under the larger torque levels. Figure 7.9 shows the locations of the crack and of the contact ellipses defined for $125 \%$ design load. 


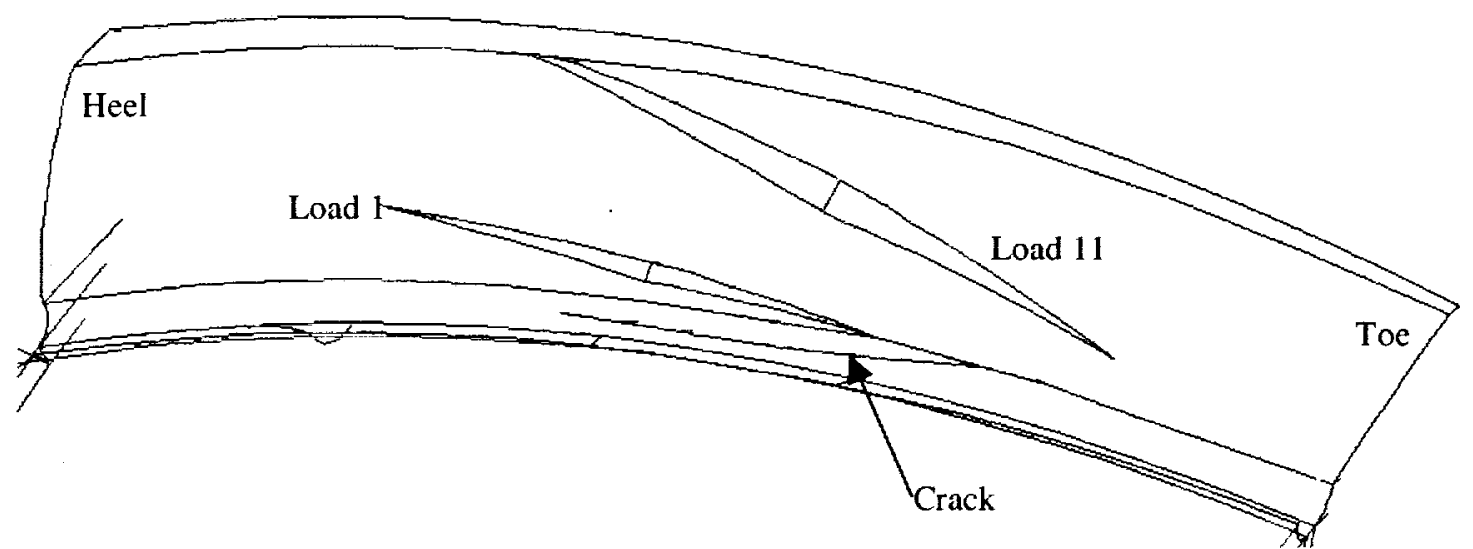

Figure 7.9: Geometry model with crack showing contact areas one and eleven defined for $125 \%$ design torque.

The mode I and II SIFs from load steps one and eleven are presented in Figures 7.10 and 7.11 , respectively. The mode I SIFs do not increase linearly with the larger loads; the $125 \%$ load has a larger effect than the $150 \%$ load. The smaller spread between the curves produced by load step one at the toe end is most likely explained by the fact that the load is not over this portion of the crack. In contrast, the SIFs increase uniformly along the entire crack front for load step eleven; the major axis of contact ellipse eleven is larger than the length of the crack mouth, and the ellipse is located directly above the crack. On the other hand, for load step one the influence of the increased load on the mode II SIFs is opposite. There is a larger spread in the curves over the portion of the crack with no load above it (toe end).

The ratio $K_{I I} / K_{I}$ is important because it determines the crack trajectory angle and the amount of rubbing between the crack faces. The larger the ratio is, the larger the kink angle will be and the greater the amount of rubbing. Figure 7.12 contains these ratios produced by the two load locations and all three load magnitudes. The curves demonstrate that the ratio of $K_{l I}$ to $K_{I}$ increases as the magnitude of load and size of the contact area increases. This implies that $K_{I I}$ is more sensitive to the changes in the torque level than $K_{l}$. This result supports the fractography observations. A large percentage of the fracture surface displayed signs of significant amounts of rubbing between the crack faces. The SIF ratios from the initial crack propagation analyses were not necessarily large enough to support the extent of rubbing observed. However, it appears that the increased torque levels will increase the amount of rubbing between the crack faces.

The kink angles calculated by the maximum principal stress theory for the various load locations and magnitudes are given in Figure 7.13. The largest absolute change in angle is $9.7^{\circ}$ and $6.4^{\circ}$ for load step one and eleven, respectively. 


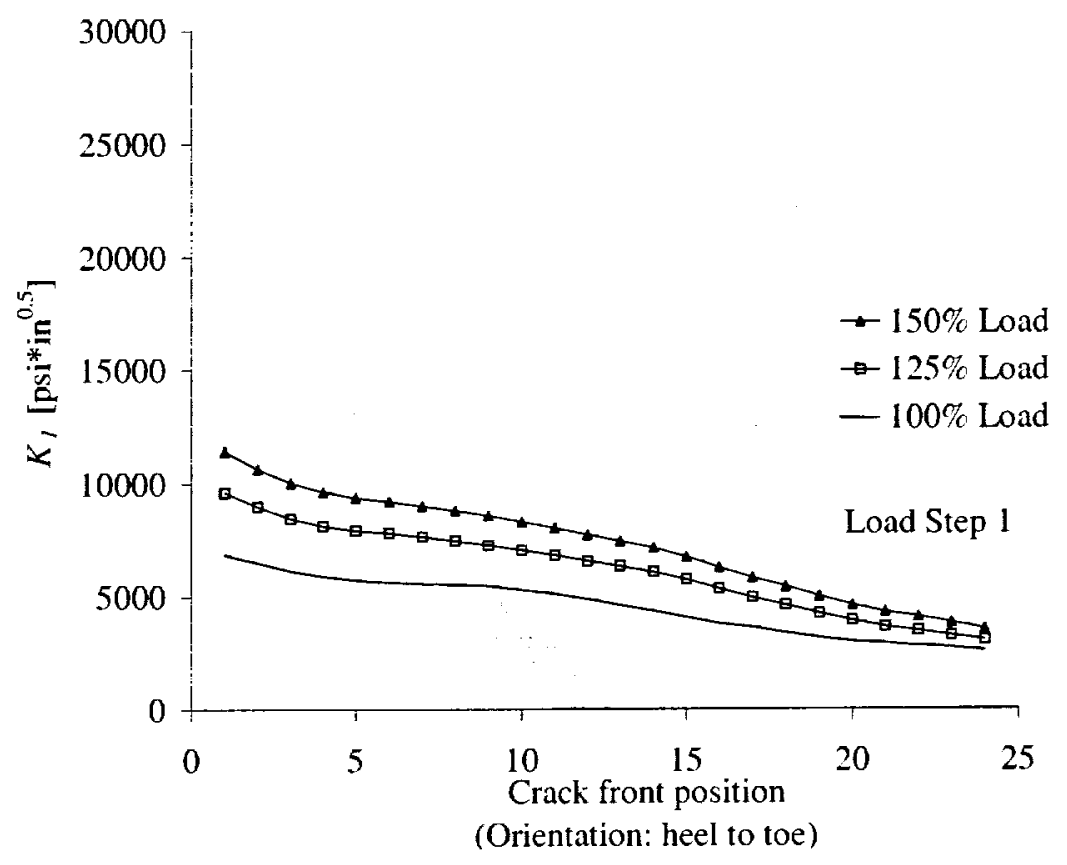

a)

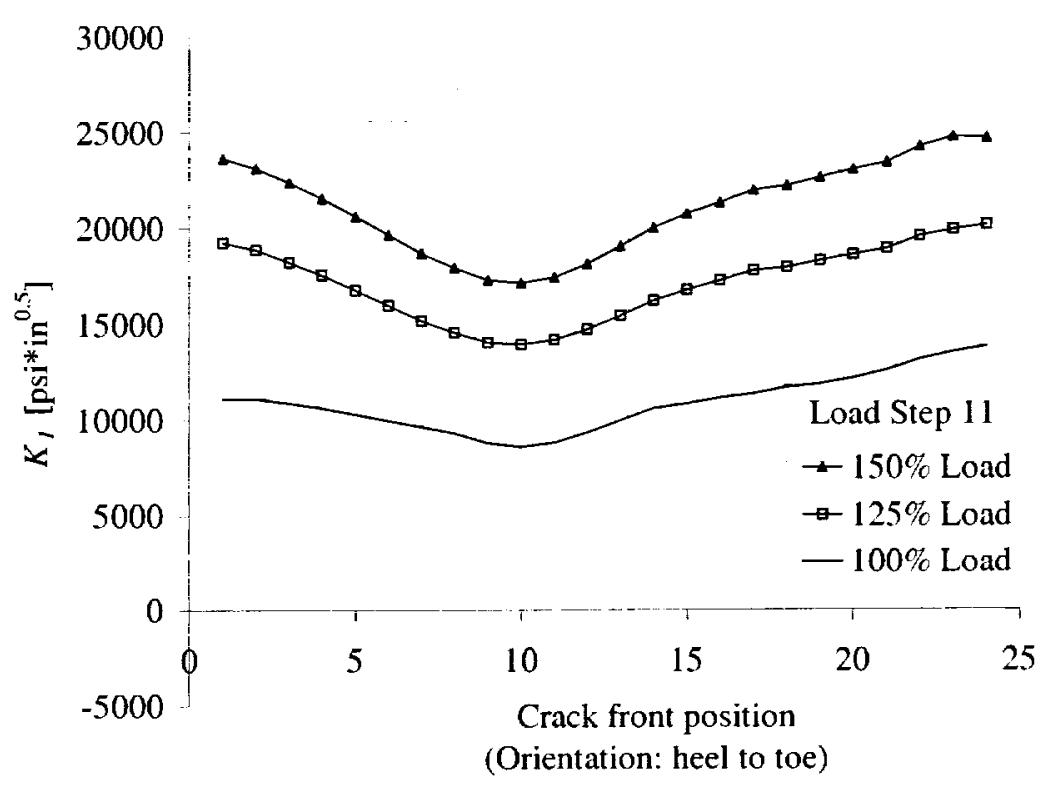

b) (Orientation: heel to toe)

Figure 7.10: $K_{I}$ distribution for load step one (a) and load step eleven (b). 


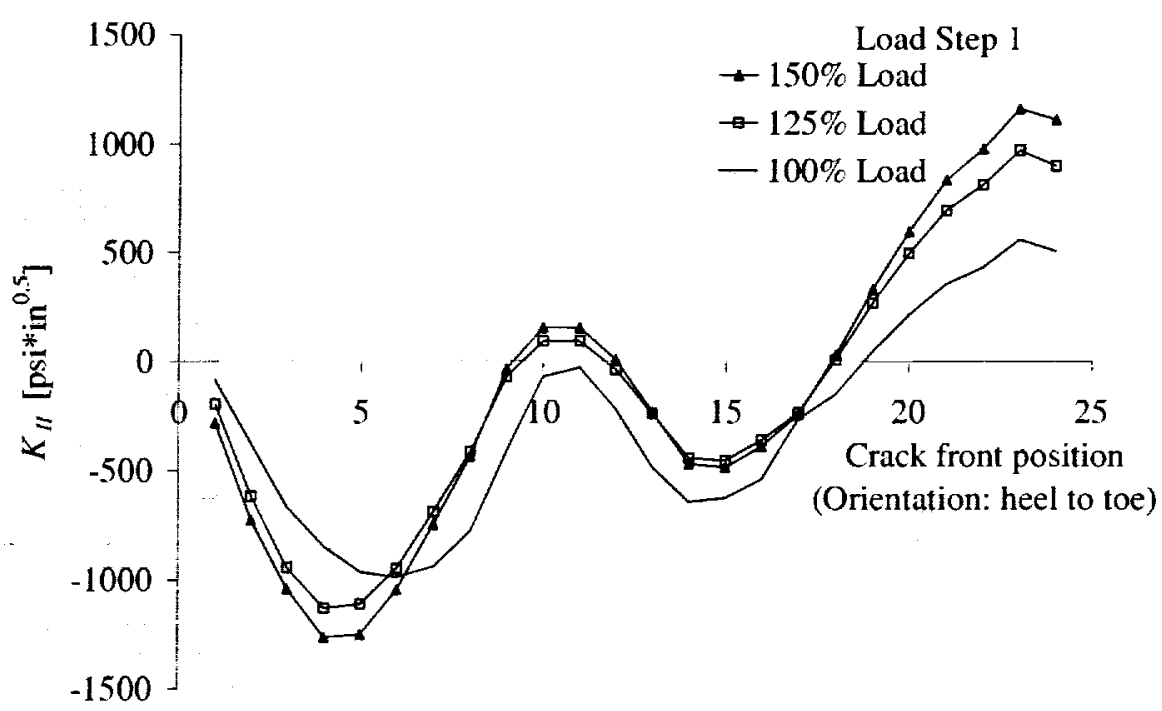

a)

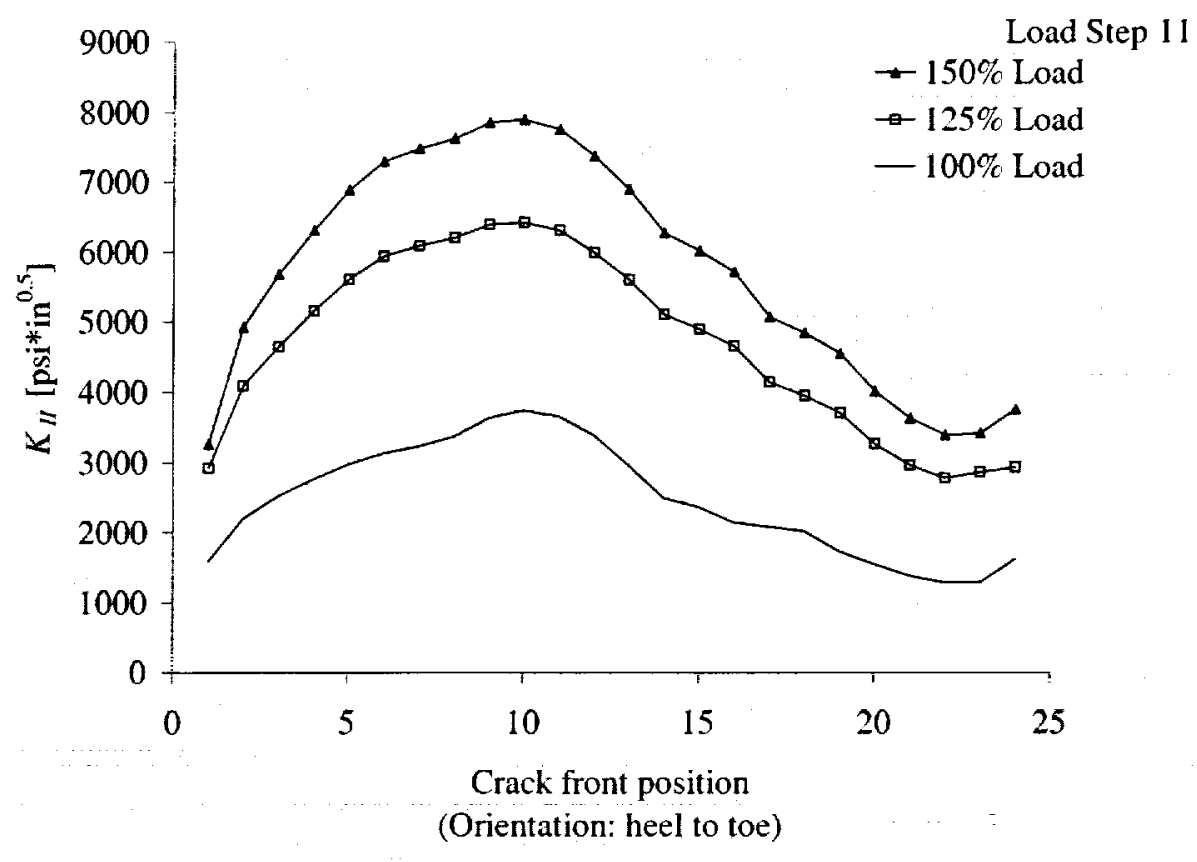

b)

Figure 7.11: $K_{I I}$ distribution for load step one (a) and load step eleven (b). 


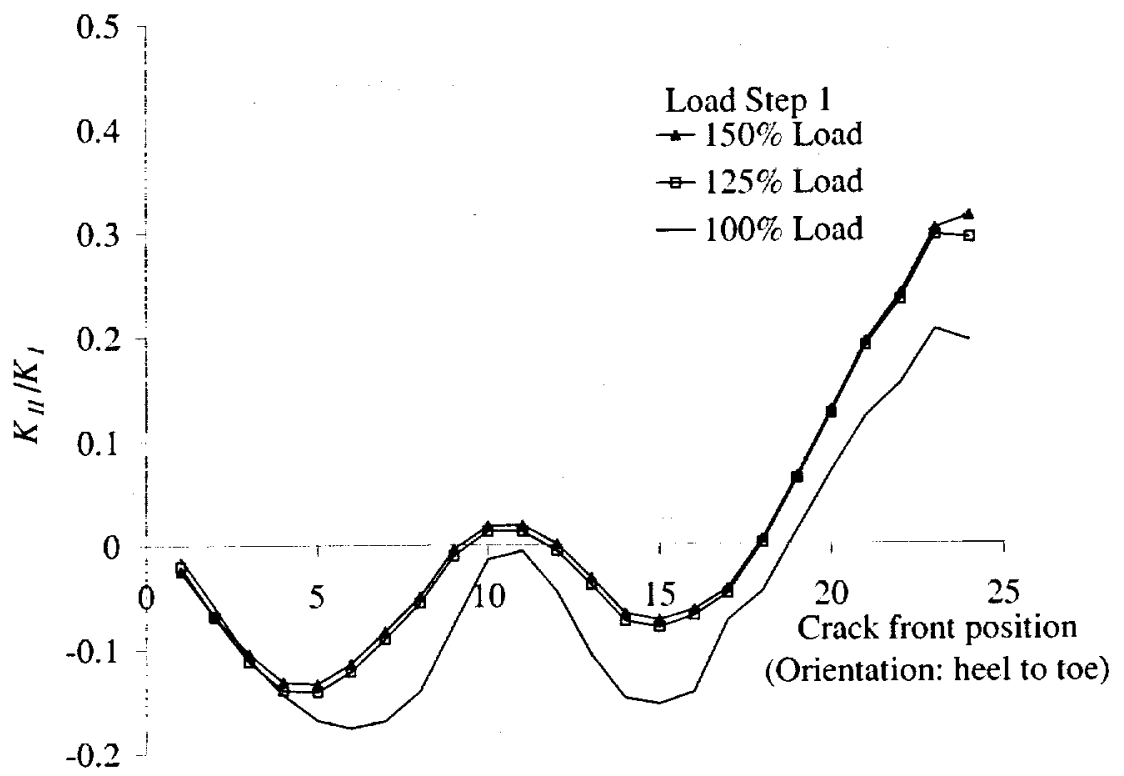

a)

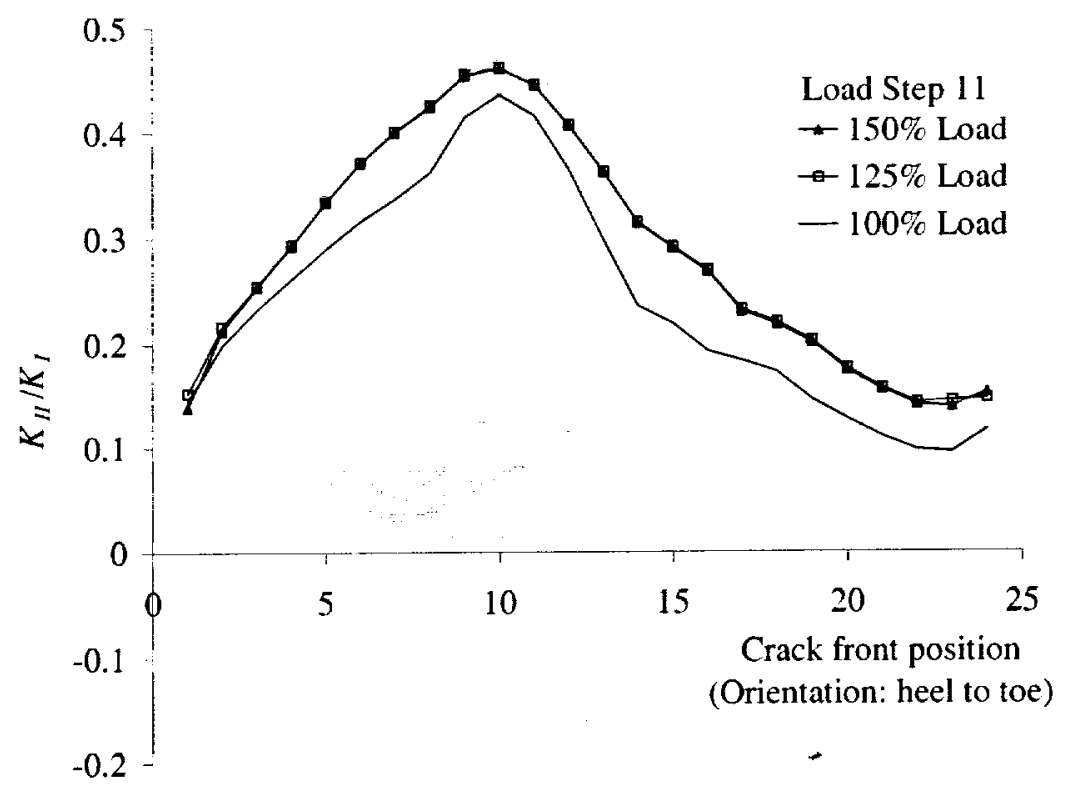

b)

Figure 7.12: $K_{I I} / K_{I}$ distribution for load step one (a) and load step eleven (b). 


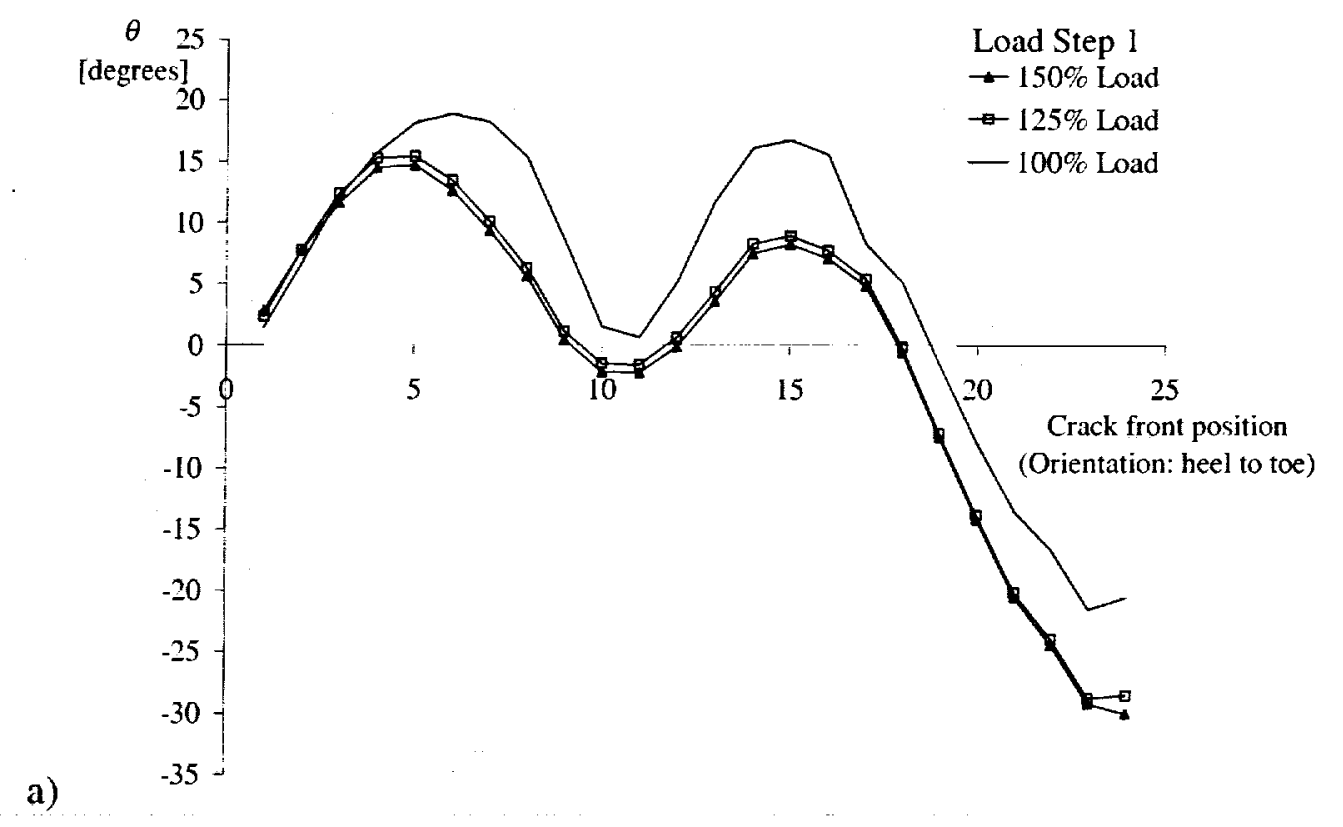

b) [degrees]

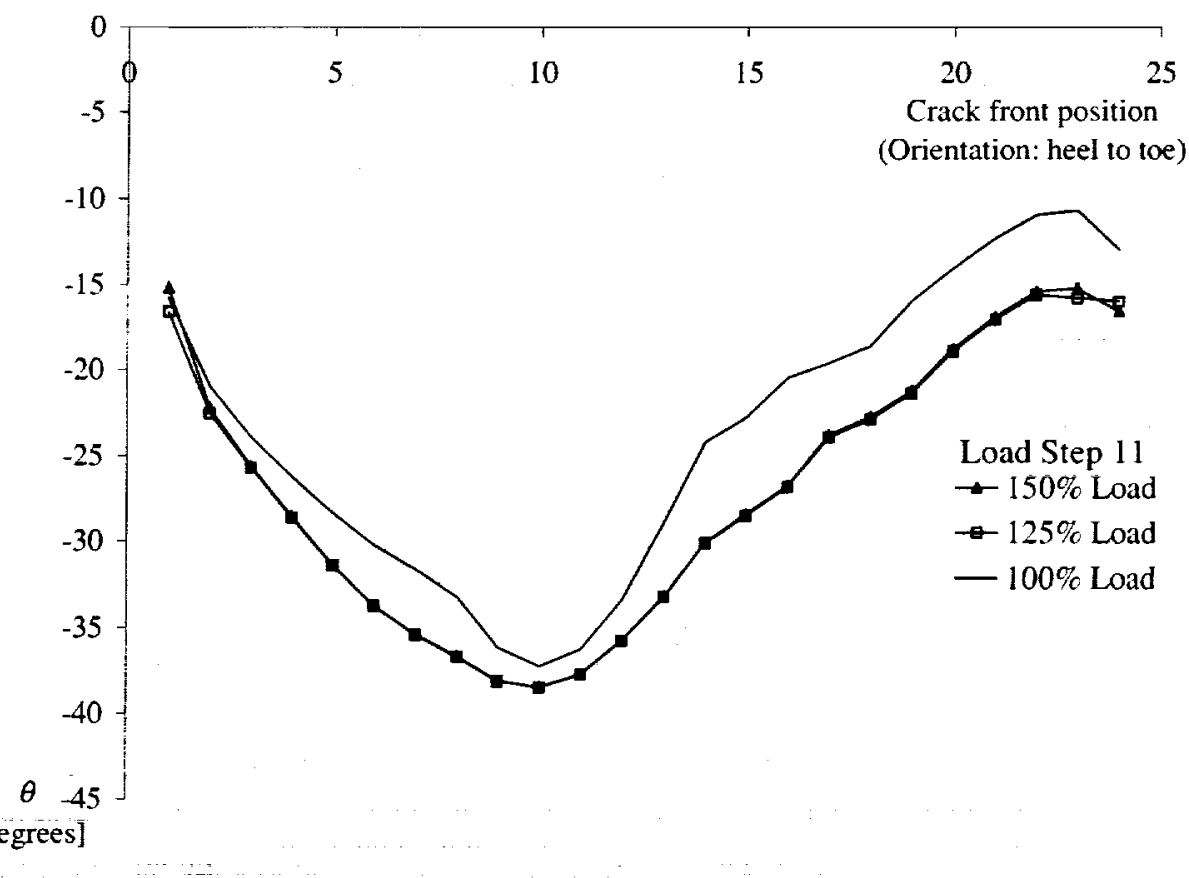

Figure 7.13: Kink angle distribution based on the maximum principal stress theory for load step one (a) and load step eleven (b).

\subsection{Highest Point of Single Tooth Contact (HPSTC) Analysis}

Comparison studies to determine the smallest model that accurately represents the gear's operating conditions were performed when developing the BEM model. These results were reported in Section 5.2. Similar comparisons are now made 
between the moving load method and a simplified loading method. Again, the assumption is that the moving load method is most accurate. The simplified method assumes a cyclic load at the HPSTC on the pinion tooth. The HPSTC is taken as contact ellipse number eleven from the discretized moving load; load step eleven is the final step of single tooth contact in the discretized load data. The magnitude of the load is defined as $100 \%$ design load. The model parameters and material properties from the moving load analyses are used in the HPSTC predictions.

The initial crack location and geometry are the same as those from the moving load analyses. The method to propagate the crack under the HPSTC is described in Section 3.2.3. The method assumes proportional loading. It is assumed that the HPSTC produces $K_{\text {Imax }}$ and that $R$ is zero. The direction of growth is determined by the maximum principal stress theory using the ratio of $K_{I I}$ to $K_{I}$ from the HPSTC loading. The extensions for the discrete crack front points are calculated with Paris' model modified to account for crack closure. Figure 7.14 is a comparison of the crack trajectories from the moving and HPSTC load methods. Roughly 190,000 cycles have occurred. The cross section view is taken at the approximate location along the tooth length of the initial crack front's midpoint.

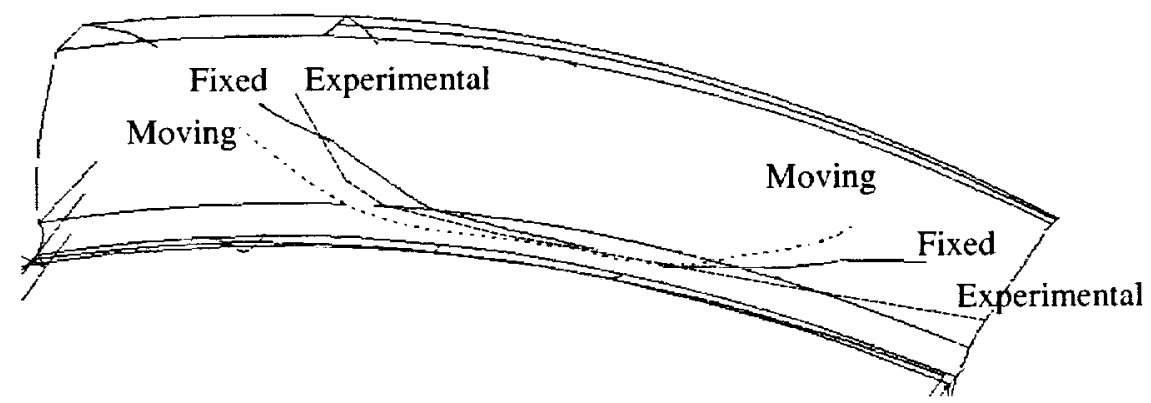

a) Tooth surface

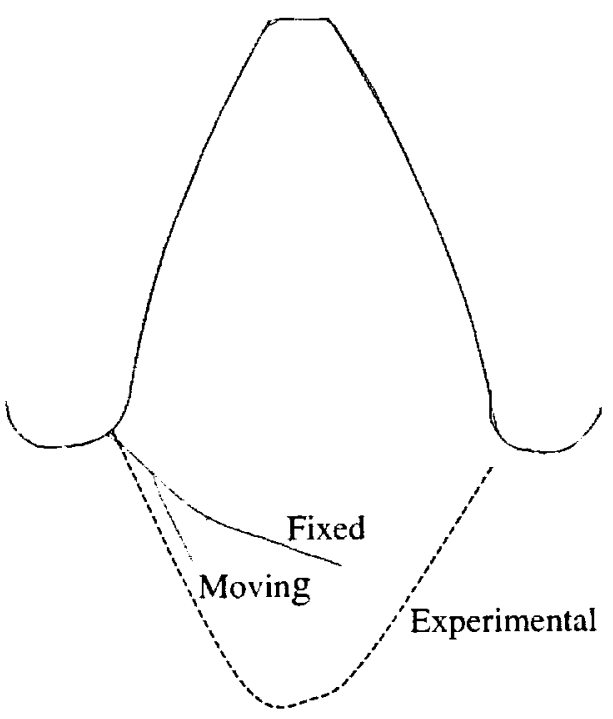

b) Cross section of tooth at midpoint of initial crack

Figure 7.14: Comparison of crack trajectories from moving load and HPSTC load (fixed location) methods after $\sim 190,000$ cycles. 
The midpoint of the crack front is deeper in the HPSTC analyses after 190,000 thousand cycles. From Figure 7.14b, it appears that the moving load analysis trajectory will produce rim failure. Figure 5.14, however, shows that the crack turns when the predictions are continued. Therefore, both the static and moving load method predict tooth failure. The slope of the trajectory into the rim in the moving load prediction matches more closely the observed trajectories in the tested pinion. This comparison is purely qualitative.

Several obvious differences between the trajectories predicted by the two methods can be observed. As seen in Figure 7.14a, the HPSTC method predicts a larger kink at the heel end; the moving load method predicts a larger kink at the toe end. Considering the location of the HPSTC load, this result is consistent with the shifted load analyses of Section 7.3.3.

One may conclude from Figure 7.14b that the HPSTC method predicts a larger crack face area since the cross section view of the crack is deeper, yet the lengths of the cracks on the tooth surface are roughly equal. Figure 7.15 , in general, supports this conclusion.

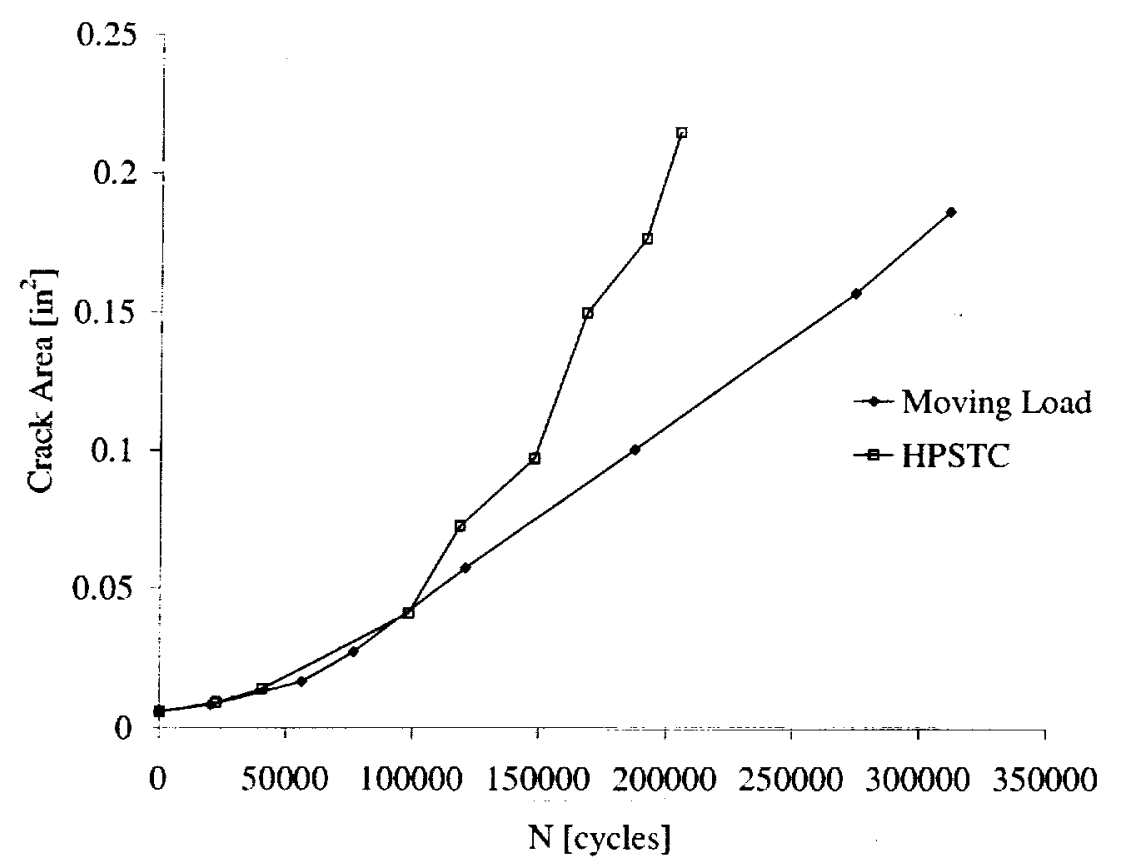

Figure 7.15: Crack area versus number of load cycles for HPSTC and moving load prediction methods.

In summary, the HPSTC analyses predict the same failure mode as the moving load analyses. The crack trajectory and fatigue life calculations vary between the two methods. Since no experimental fatigue life data exists, the accuracy of one methods fatigue life prediction over the other methods can not be evaluated. The moving load 
predictions match the experimental trajectory into the rim and through the cross section of the tooth better than the HPSTC prediction. Since the trajectory into or through the rim is what determines tooth failure or rim failure, it is concluded that the moving load method is necessary to capture that result most accurately. All of the trajectories on the tooth surface at the heel end, however, are in reasonable agreement. Nonetheless, a distinct advantage of the HPSTC method is the significant decrease in computational time to perform the crack propagation predictions since only one load case needs to be analyzed.

\subsection{Chapter Summary}

The results from a fatigue crack growth simulation in a spiral bevel pinion were compared to crack growth observations in a tested pinion. The comparisons are summarized as follows:

- The simulations predicted a reasonable fatigue life with respect to the test data.

- The original trajectory predictions failed to capture detailed aspects of the observed fracture surfaces in the test. It was determined that the simulated loading on the tooth probably modeled the tooth contact in the test incorrectly. The tooth contact information used in the predictions assumed perfect alignment between the pinion and the gear and that the gears were not flawed. Some explanations for the differences in contact between the test and theory were determined to be:

1. Change in contact location in the test as the crack grew and the tooth became more flexible.

2. Differences in the magnitude of loading.

3. Crack growth under load control (simulation) versus displacement control (test).

4. Misalignment between the gear and pinion in the test.

- Additional simulations demonstrated the capability to predict the crack trajectory observed in the test. A large initial crack, which was assumed to approximate the location of the crack front just prior to the formation of the ridge, was used and the crack was propagated through a series of steps.

Sensitivity studies were conducted to determine how changes in some of the crack growth method's assumptions would modify the predictions. The studies determined that:

- The fatigue crack growth rate model parameters used in the initial prediction yielded conservative results.

- The crack front condition is best described as plane strain.

- A reasonable approximation of the dimensionless quantity $\beta$, which incorporates geometry effects when calculating SIFs, is a value of 1 .

- The trajectory observed in the tested pinion would result from a contact biased toward the toe end.

- The increased torque levels might explain the significant amounts of rubbing seen on the fracture surfaces of the tested pinion. 
A simplified loading method that assumes a cyclic load at the HPSTC on the pinion tooth during meshing was investigated. The failure mode predicted by this method was the same as the moving load predictions. However, the crack trajectory and fatigue life calculations varied between the two methods. The HPSTC method is advantageous because it significantly reduces the computational time. However, upon comparison of the results from the two methods to experimental results, it is concluded that the moving load method's trajectories are more accurate.

In summary, insights into the intricacies of modeling fatigue crack growth in three dimensions were gained. Preliminary steps toward accurately modeling crack growth in complicated three dimensional objects such as spiral bevel gears were completed successfully. To improve the accuracy of the simulations, the change in contact between spiral bevel gear teeth during operation as a crack evolves is needed. 


\section{CHAPTER EIGHT: CONCLUDING REMARKS}

\subsection{Accomplishments and Significance of Thesis}

This thesis investigated computationally modeling fatigue crack growth in spiral bevel gears. Predicting crack growth is significant in the context of gear design because a crack's trajectory determines whether the failure will be benign or catastrophic. Having the capability to predict crack growth in gears allows a designer to prevent catastrophic failures. Prior to this thesis, numerical methods had been limited to modeling cracking in gears with simpler geometry, such as spur gears. Spur gear geometry permits the use of two dimensional analyses. However, spiral bevel gears require a three dimensional model of the geometry and cracks. Three dimensional models are much more complicated to create, require greater computing power because of the significant increase in degrees of freedom, and no closed form solutions exist to predict the growth of arbitrary three dimensional cracks. Prior to this thesis, few predictions of crack growth in spiral bevel gears had been performed. Accurately modeling three dimensional fatigue crack trajectories in a spiral bevel pinion required expanding the state-of-the-art capabilities and theories for predicting fatigue crack growth rates and crack trajectories.

The geometry of a spiral bevel pinion from the transmission system of the U.S. Army's OH-58 Kiowa Helicopter was used for demonstrative purposes. A BEM model of the pinion was developed using a computer program developed by NASA/GRC that calculates the surface coordinates of a spiral bevel gear tooth. Their tooth contact analysis program was also used to determine the location, orientation, and magnitude of contact between the pinion and its mating gear. The contact was represented by discrete traction patches on the gear tooth.

LEFM theories were combined with the BEM to accomplish the crack growth predictions. The simulations were based on computational fracture mechanics software developed by the Cornell Fracture Group, which allow for arbitrarily shaped, three dimensional curved crack fronts and crack trajectories. The crack trajectories were determined by a Paris model, modified to incorporate crack closure, to calculate fatigue crack growth rates in conjunction with the maximum principal stress theory to calculate kink angles.

In operation, the load on a gear tooth varies over time in location and magnitude. This moving load effect was incorporated into the propagation method. Only loads normal to a gear tooth's surface were considered. It was discovered that the moving normal load produces a non-proportional load history in the tooth root. Proposed prediction methods for fatigue crack growth under non-proportional loads in the literature were determined to be insufficient for the spiral bevel gear model. As a result, a method to predict three dimensional fatigue crack growth under nonproportional loading was developed. The method incrementally advanced the crack front for a series of discrete load steps throughout one load cycle. A number of load cycles were then specified, and the crack was advanced an amount based on the number of specified load cycles and the calculated trajectory from the single load cycle; the process was then repeated. Some aspects of the final crack trajectory 
predicted by this moving load method differed from a failure in a tested pinion; however, the method succeeded in predicting a fatigue life comparable to the experimental data.

Other issues related to modeling crack growth in a gear were also investigated. For example, the effect of shifting the load location along a tooth's length on the crack trajectories was confirmed. For a crack that has initiated in the tooth's root, when the load location is directly above the crack, the crack trajectory will remain very close to the root. Additionally, the effect of compressive loads on fatigue crack growth rates in AISI 9310 steel was examined. This examination is significant because a principal focus of current gear design is to minimize a gear's weight. Reducing the amount of material in the gear may increase the magnitude of the compressive stresses in a gear tooth's root, which could influence crack growth rates. It was discovered that the compressive portion of a load cycle did not significantly modify the rates when crack closure was incorporated into Paris' model to calculate fatigue crack growth rates. As a result, the BEM/LEFM analyses of a spiral bevel pinion were carried out ignoring the compressive portions of the loading history.

The predictions from the moving load crack propagation method were compared to predictions when only HPSTC was considered. HPSTC is a more simplified approach and has been commonly used in past research when numerically analyzing crack propagation in gears. The HPSTC method utilized existing fatigue crack growth theories since there was a single load location and proportional loading. The analyses in this thesis with the two loading methods predicted different fatigue lives and crack trajectories. The lack of experimental fatigue crack growth rate data hindered an evaluation of the crack growth rates predicted by the two methods. The moving load method's crack trajectory predictions agreed more closely to the tested pinion failures. Crack trajectories are of primary importance to predict the failure mode.

The dearth of fatigue crack growth rate data and crack front shape information from tooth failures in a tested spiral bevel pinion motivated SEM observations of the fracture surfaces. A crack growth scenario was devised from the observations. In addition, the observations suggested that the failure mechanism along the majority of the surface was fatigue. This result supported the use of the numerical simulations to predict fatigue crack growth trajectories in the gear.

As this thesis was a first attempt at predicting fatigue crack growth in spiral bevel gears, certain limitations were encountered. The limitations can be summarized as follows:

- A scarcity of experimental data prohibited validations of calculated crack growth rates, fatigue life predictions, and crack front shape evolution.

- The effect of tooth deflections on the contact area between mating gear teeth was not modeled. Capturing this effect will increase the accuracy of the model since crack trajectories are strongly determined by the load locations.

- It is anticipated that the deflections of a cracked spiral bevel gear tooth will be limited by the adjacent tooth picking up the load. The magnitude of this 
maximum deflection is unknown. As a result, the simulations did not model this behavior.

- The method to predict crack trajectories under the moving load was time consuming since every discrete load step had to be analyzed for a full pinion model. For the method to be more practical to a gear designer, improvements in the computational time and implementation would have to be made.

In summary, the achievements of this thesis brought the current gear design approach closer to developing a numerical tool to evaluate safety aspects of gear geometries. Critical areas that must be understood in greater detail prior to predicting more accurate crack trajectories and growth rates in three dimensions were identified. By incorporating non-proportional loading, complex three dimensional geometry, and arbitrary three dimensional crack models, the work extended the current capabilities and theories for predicting fatigue crack growth rates and crack trajectories.

\subsection{Recommendations for Future Research}

The accomplishments of this thesis produced many new questions and issues related to simulating fatigue crack growth in spiral bevel gears. Future research focused on the following areas will further assist the development of numerical design tools to evaluate a gear design's safety.

- A testing program centered on fatigue crack growth rates and trajectories from non-proportional loads should be carried out. A more fundamental understanding of crack behavior in this type of loading environment is needed to enhance the accuracy of fatigue crack growth rate models. To gain insights into the fundamental behavior, the tests do not have to be conducted with spiral bevel gears. To judge the correctness of the predictions in this thesis, however, more tests on spiral bevel gears that record crack growth rates and trajectories over a time period are necessary. Such test data are essential to confirm the accuracy of the simulations and to evaluate the proposed method for predicting crack growth under the moving tooth load.

- A more detailed understanding of the contact between a cracked gear tooth mating with an uncracked gear tooth is required. The load redistribution effects in this scenario could be studied either experimentally or numerically. Numerical studies would require a fully three dimensional, LEFM, and contact analysis of the rolling process between two mating gears. Once the redistribution effects are captured, the findings could be applied to crack growth simulations. The expectation is that the simulations would model more accurately the observed behavior in real failures.

- The analyses in this thesis considered only loads normal to the tooth surface. However, a gear tooth is subjected to a variety of loads in operation. For example, the contact between the mating gears also produces frictional forces along with the normal loads over the contact ellipse area. Gears in rotorcraft applications operate at elevated temperature, and, therefore, thermal effects might be included. In addition, the rotation of the gear produces centrifugal forces. Dynamic loads are 
produced from the contact between the mating gears in conjunction with the high loading frequency. Several researchers have also modeled the residual stresses in a gear, which result from the difference in hardness between the case and core, in numerical analyses by thermal loads. Parametric studies to determine the significance of all these loading variables on predicted fatigue crack growth in a spiral bevel gear is imperative to the development of numerical gear design tools.

- Further work to enhance the speed of the numerical analyses and increase the SIF accuracy would be beneficial. One method to improve the accuracy is to use the FEM. However, meshing three dimensional volumes with cracks introduces additional difficulties. Research is currently underway, and should be continued, to overcome these meshing difficulties. The computational time will continue to decrease as computer technology rapidly advances. The overall objective of developing a practical and accurate numerical design tool for any type of gear is foreseeable in the near future. 


\section{APPENDIX A}

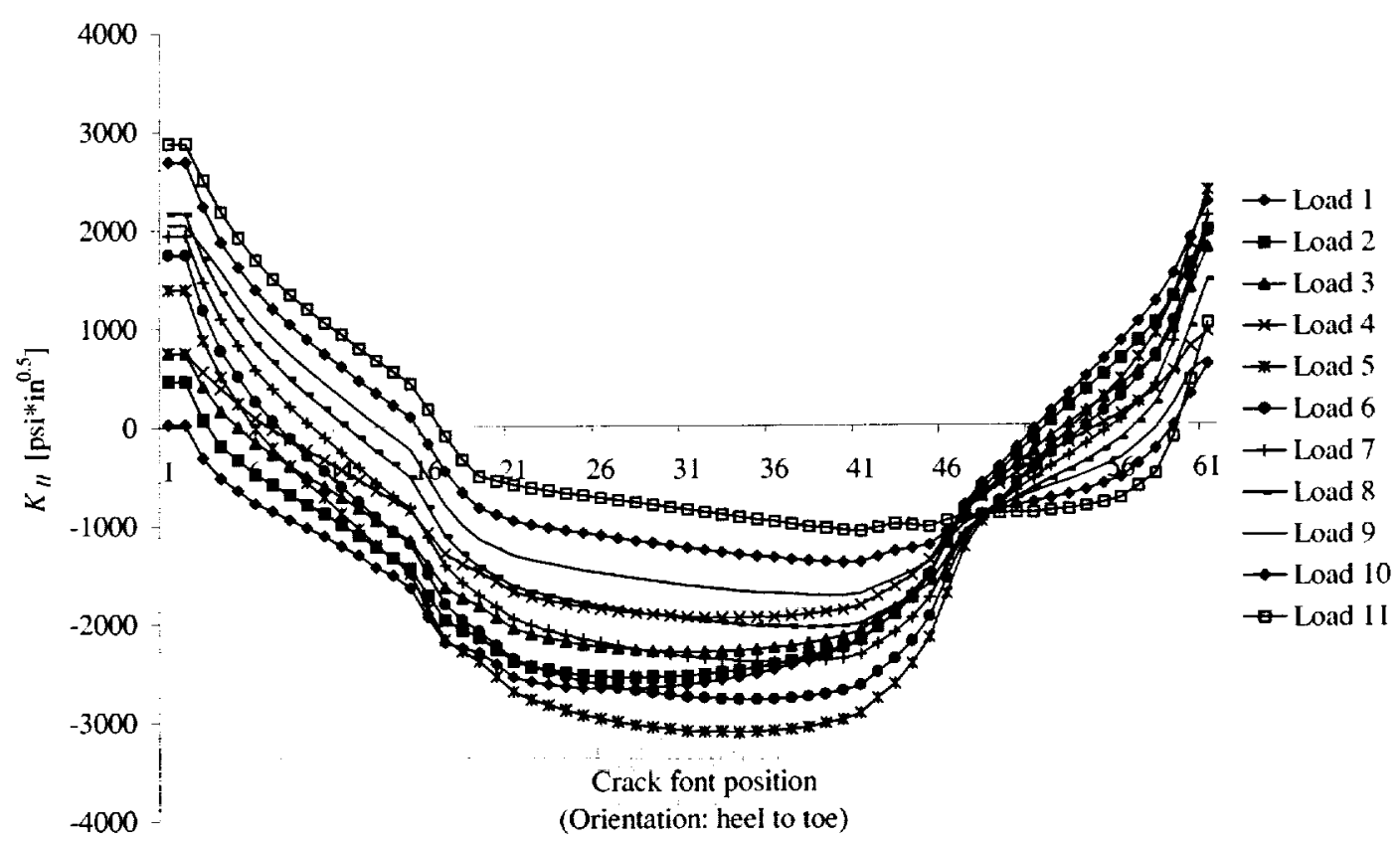

Figure A.1: Mode II SIF distribution for load steps one through eleven.

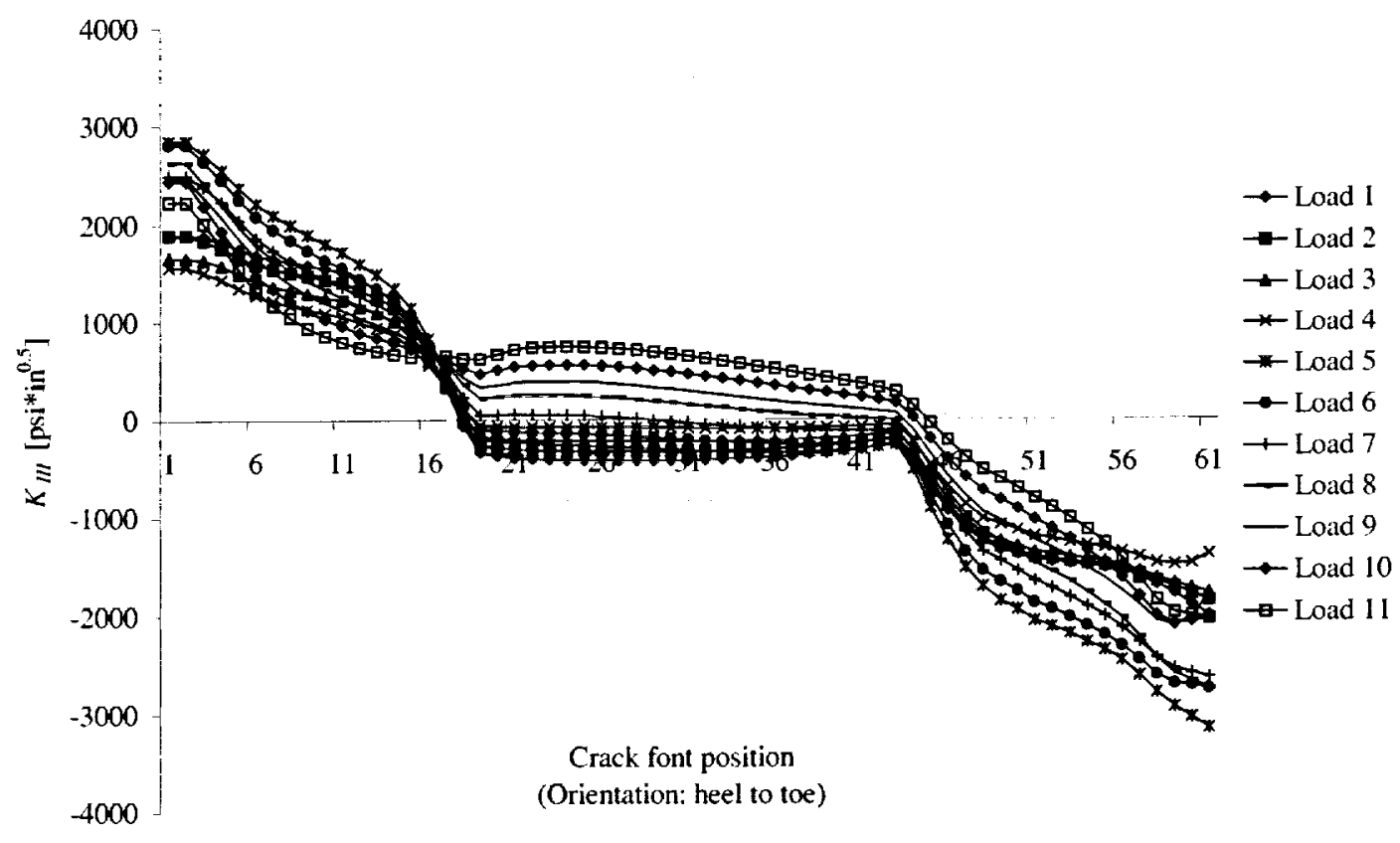

Figure A.2: Mode III SIF distribution for load steps one through eleven. 


\section{APPENDIX B}

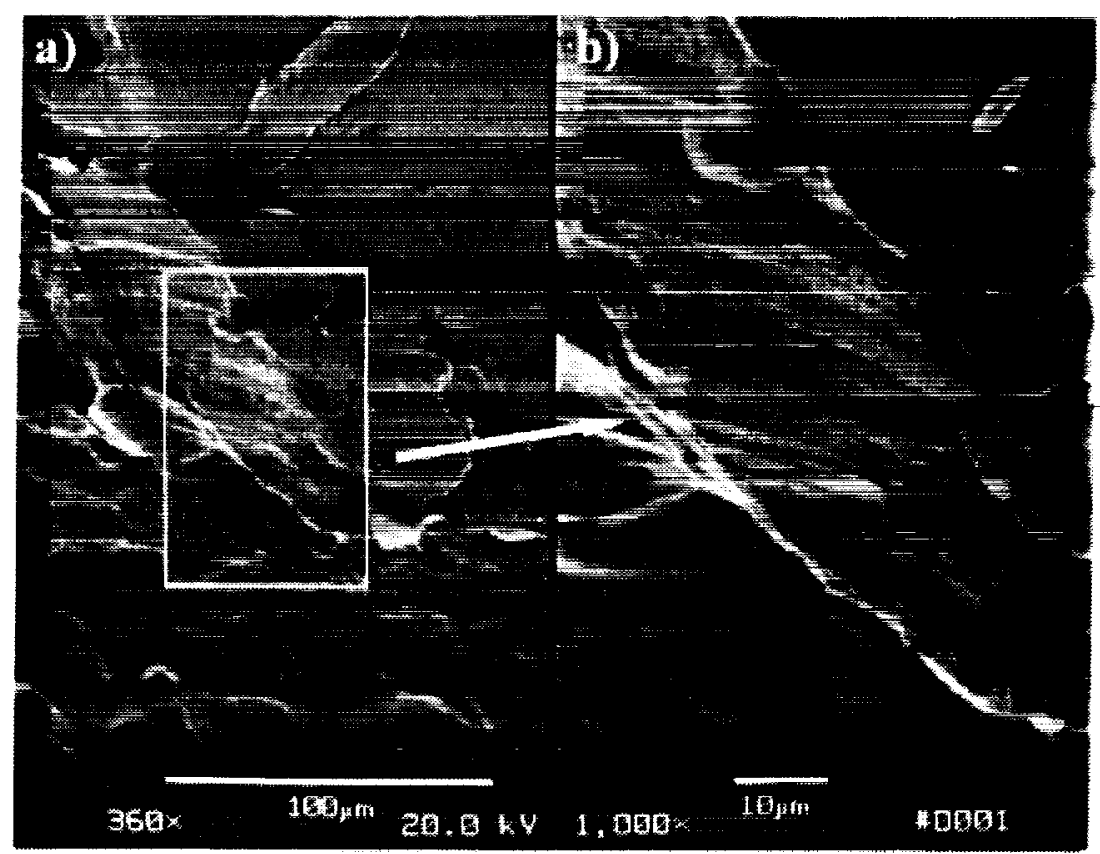

Figure B.1: Fatigue striations in Figure 6.5 at $360 \times(a)$ and $1,000 \times(b)$.

An additional point of interest is captured in Figure B.3. The figure is of the tooth surface. The horizontal lines are the grooves from the machining of the gear. In addition, two pits are observed on the surface. These pits could have resulted from several variables such as particles that were caught on the surface during meshing of the teeth, manufacturing flaws, surface wear, etc. The cuts and pits give an indication of the initial flaw size from which fatigue cracks may originate. 


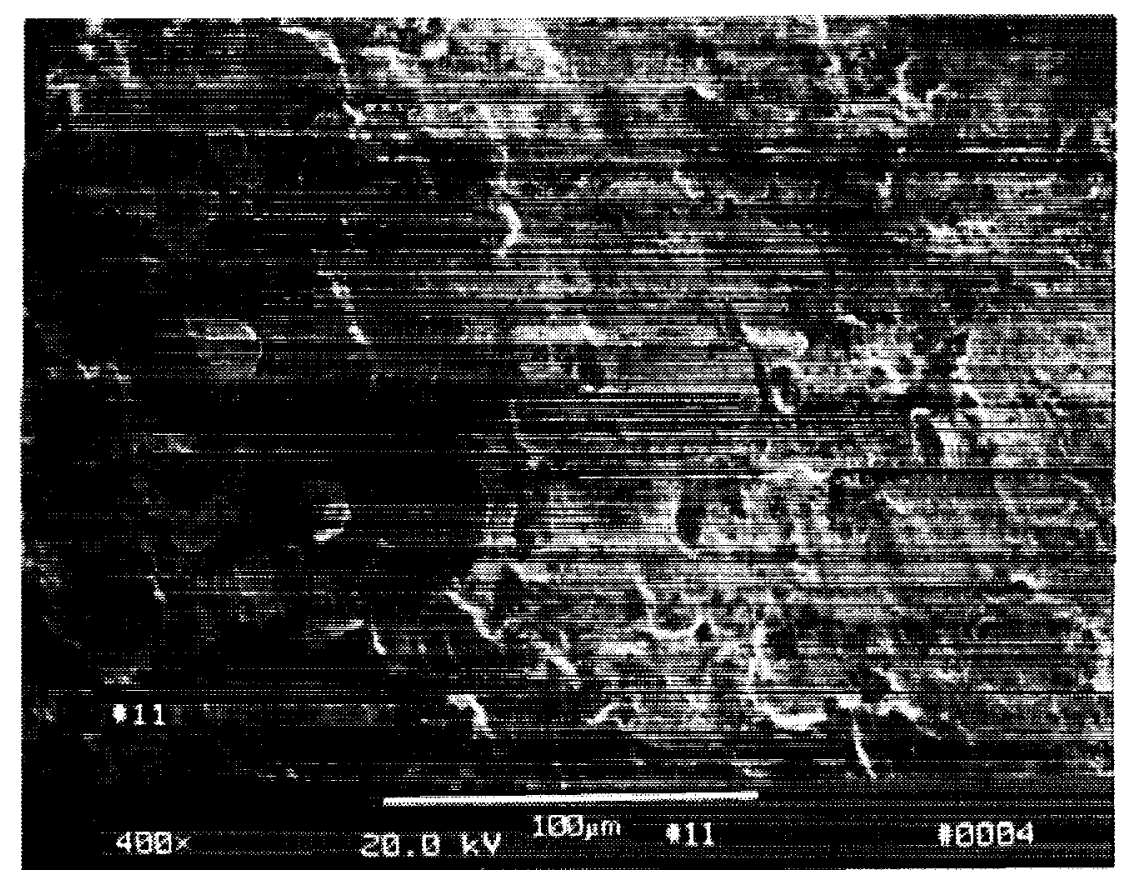

Figure B.2: Typical picture of flat, polished area on tooth \#1 1 (400x). Photograph was taken near location B in Figure 6.4.

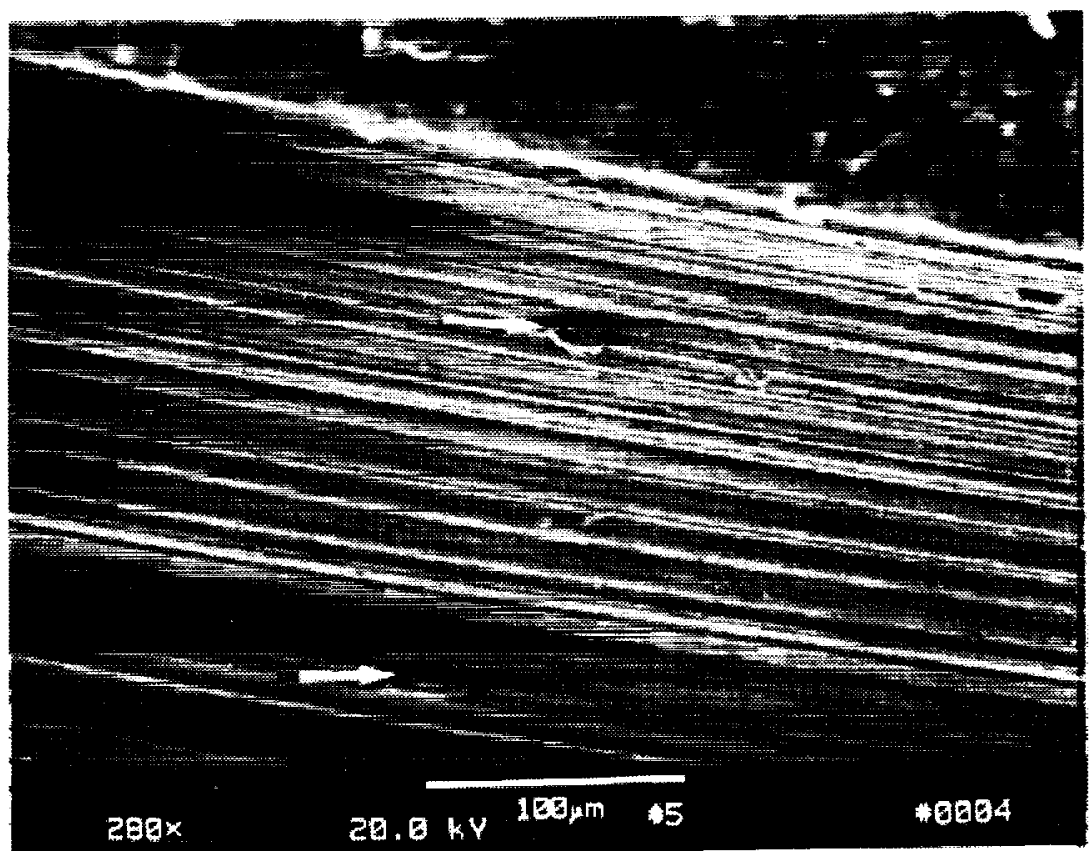

Figure B.3: Machining grooves and pits on tooth surface (280×). 


\section{APPENDIX C}

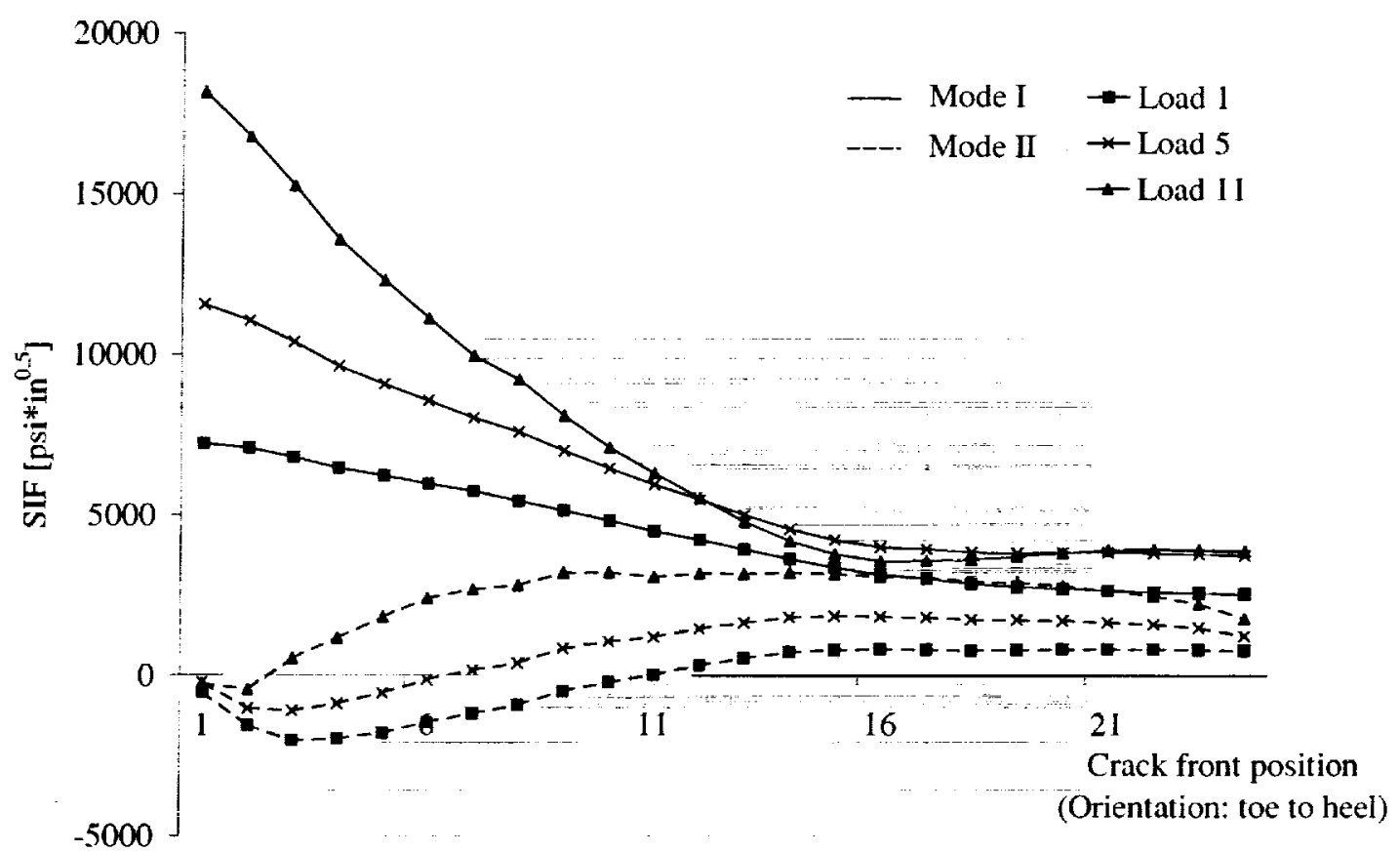

Figure C.1: SIFs from toe-shifted load steps one, five, and eleven.

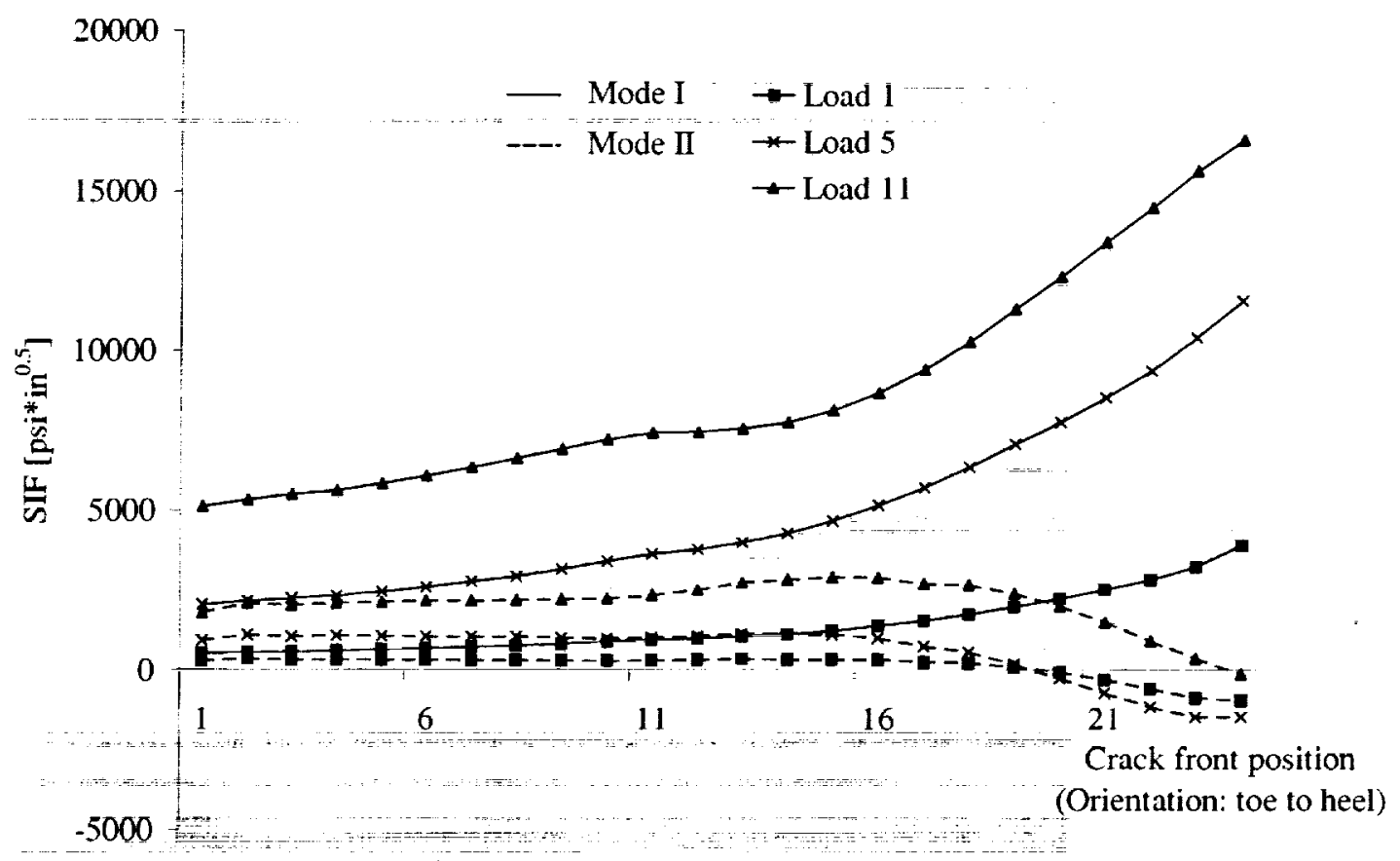

Figure C.2: SIFs from heel-shifted load steps one, five, and eleven. 


\section{REFERENCES}

AGMA 1983

AGMA 1996

Alban 1985

Alban 1986

Albrecht 1988

AMS 1996

ASTM 1997

Bibel et al. 1995

Bibel et al. 1996

Binder et al. 1980

Bingyuan et al. 1991
Au et al. 1981

AGMA 246.02A, 1983, "Practice for Carburized Aerospace Gearing," American Gear Manufacturers Association, Alexandria, VA.

ANSI/AGMA 2005-C96, 1996, "Design Manual for Bevel Gears," American Gear Manufacturers Association, Alexandria, VA.

Alban, L.E., 1985, Systematic Analysis of Gear Failures, American Society for Metals.

Alban, L.E., 1986, "Failures of Gears," Metals Handbook, $9^{\text {th }}$ Edition., Volume 11, Failure Analysis and Prevention, ASM International, pp. 586-601.

Albrecht, C., 1988, "Transmission Design Using Finite Element Method Analysis Techniques," Journal of the American Helicopter Society, Volume 33, no. 2, pp. 3-14.

AMS 6265J, 1996, Aerospace Material Specification, SAE International, Pennsylvania.

ASTM E 399-90, 1997, "Standard Test Method for Plane-Strain Fracture Toughness of Metallic Materials," Annual Book of ASTM Standards, Metals Test Methods and Analytical Procedures, Volume 03.01, pp. 408-438.

$\mathrm{Au}$, J.J., and Ke, J.S., 1981, "Correlation Between Fatigue Crack Growth Rate and Fatigue Striation Spacing in AISI 9310 (AMS 6265) Steel," Fractography and Materials Science, ASTM STP 733, L.N. Gilbertson and R.D. Zipp, Eds., American Society for Testing and Materials, pp. 202-221.

Bhattacharyya et al. 1979 Bhattacharyya, S., Johnson, V.E., Agarwal, S., and Howes, M.A.H., Eds., 1979, IITRI Fracture Handbook, Failure Analysis of Metallic Materials by Scanning Electron Microscopy, The Materials Research Division IIT Research Institute, Chicago, Illinois.

Bibel, G.D., Kumar, A., Reddy, S., and Handschuh, R., 1995, "Contact Stress Analysis of Spiral Bevel Gears Using Finite Element Analysis," Journal of Mechanical Design, Volume 117, pp. 235-240.

Bibel, G.D., and Handschuh, R., 1996, "Meshing of a Spiral Bevel Gearset with 3D Finite Element Analysis," NASA Technical Memorandum 107336/ARL-TR-1224.

Binder, S., and Mack, J.C., 1980, "Experience with Advanced High Performance Gear Steel," ASME Paper No. 80-C2/DET-77.

Bingyuan, J., Xitong, Z., and Lei, W., 1991, "Estimate by Fracture Mechanics Theory for Rolling Contact Fatigue Life and Strength of 
Bingyuan et al. 1991

Blarasin et al. 1997

Blom et al. 1985

Bold et al. 1992

Bower et al. 1994

Broek 1986

Chan et al. 1970

Couchon et al. 1993

Coy et al. 1985

Coy et al. 1987

Dike 1978

Dudley 1996
Bingyuan, J., Xitong, Z., and Lei, W., 1991, "Estimate by Fracture Mechanics Theory for Rolling Contact Fatigue Life and Strength of Case-Hardened Gear Materials with Computer," Engineering Fracture Mechanics, Volume 39, pp. 867-874.

Blarasin, A., Guagliano, M., and Vergani, L., 1997, "Fatigue Crack Growth Predictions in Specimens Similar to Spur Gear Teeth," Fatigue and Fracture of Engineering Materials and Structures, Volume 20, pp. 1171-1182.

Blom, A.F., and Holm, D.K., 1985, "An Experimental and Numerical Study of Crack Closure," Engineering Fracture Mechanics, Volume 22, pp. 997-1011.

Bold, P.E., Brown, M.W., and Allen, R.J., 1992, "A Review of Fatigue Crack Growth in Steels Under Mixed Mode I and II Loading," Fatigue and Fracture of Engineering Materials and Structures, Volume 15, pp. 965-977.

Bower, A.F., and Fleck, N.A., 1994, "Brittle Fracture Under A Sliding Line Contact," Journal of the Mechanics and Physics of Solids, Volume 42, pp. 1375-1396.

Broek, D., 1986, Elementary Engineering Fracture Mechanics, Fourth revised edition, Kluwer Academic Publishers.

Chan, S.K., Tuba, I.S., and Wilson, W.K., 1970, "On the Finite Element Method in Linear Fracture Mechanics," Engineering Fracture Mechanics, Volume 2, pp. 1-17.

Couchon, D.C., Barnes, G.K., Cedoz, R.W., 1993, Shot-Peened Gear Failures Due to Operation in a Misaligned Condition, AIAA 93-2147.

Coy, J.J., Townsend, D.P., and Zaretsky, E.V., 1985, "Gearing," NASA Reference Publication 1152/AVSCOM Technical Report 84-C15.

Coy, J.J., Handschuh, R.F., Lewicki, D.G., Hugg, R.G., Krejsa, E.A., and Karchmer, A.M., 1987, "Identification and Proposed Control of Helicopter Transmission Noise at the Source," USAAVSCOM-TR-87C-2.

Dike, G., 1978, Geometry and Stresses of Straight Bevel Gears, Transactions of Machine Elements Division, Lund Technical University, Lund, Sweden.

Dudley, D.W., 1996, "Fatigue and Life Predictions of Gears," ASM Handbook Volume 19: Fatigue and Fracture, ASM International, pp. 345-354. 
El-Zafrany 1993

Elber 1971

Erdogan et al. 1963

Flasker et al. 1993

Fleck et al. 1988

Forman et al. 1984

Forsyth 1962

FRANC3D 1999a

FRANC3D 1999b

FRANC3D 1999c

Fu et al. 1995
El-Zafrany, A., 1993, Techniques of the Boundary Element Method, Ellis Horwood Limited.

Elber, W., 1971, "The Significance of Fatigue Crack Closure," Damage Tolerance in Aircraft Structures, ASTM STP 486, American Society for Testing and Materials, pp. 230-242.

Erdogan, F., and Sih, G.C., 1963, "On the Crack Extension in Plates Under Plane Loading and Transverse Shear," Journal of Basic Engineering, Volume 85, pp. 519-527.

Flasker, J., and Pehan, S., 1993, "Crack Propagation in Tooth Root with Variable Loading," Communications in Numerical Methods in Engineering, Volume 9, pp. 103-110.

Fleck, N.A., and Newman, J.C., Jr., 1988, "Analysis of Crack Closure Under Plane Strain Conditions," Mechanics of Fatigue Crack Closure, ASTM STP 982, J.C. Newman, Jr. and W. Elber, Eds., American Society for Testing and Materials, pp. 319-341.

Forman, R.G., and Hu, T., 1984, "Application of Fracture Mechanics on the Space Shuttle," Damage Tolerance of Metallic Structures: Analysis Methods and Applications, ASTM STP 842, J.B. Chang and J.L. Rudd, Eds., American Society for Testing and Materials, pp. 108133.

Forsyth, P.J.E, 1962, "A Two Stage Process of Fatigue Crack Growth," Proceedings of the Crack Propagation Symposium, Volume 1, pp. 76-94.

FRANC3D VI.14 Concepts \& Users Guide, 1999, developed by the Cornell Fracture Group, Cornell University, Ithaca, NY.

FRANC3D VI.14 Menu \& Dialog Reference, 1999, developed by the Cornell Fracture Group, Cornell University, Ithaca, NY.

FRANC3D V1.14 Validation \& Verification Reference, 1999, developed by the Comell Fracture Group, Cornell University, Ithaca, NY.

Fu, M., and Shang, B., 1995, "Stress Analysis of the Precision Forging Die for a Bevel Gear and its Optimal Design Using the BoundaryElement Method," Journal of Materials Processing Technology, Volume 53, pp. 511-520. 
Glodez et al. 1998

Griffith 1921

Handschuh et al. 1991

Hourlier et al. 1985

Irving et al. 1999

Irwin 1957

Jeong et al. 1997

Johnson 1985

Kurihara et al. 1986

Laird 1967

Lewicki et al. 1993
Glodez, S., Pehan, S., and Flasker, J., 1998, "Experimental Results of the Fatigue Crack Growth in a Gear Tooth Root," International Journal of Fatigue, Volume 20, pp. 669-675.

Griffith, A.A., 1921, "The Phenomena of Rupture and Flow in Solids," Philosophical Transactions of the Royal Society of London, Volume A221, pp. 163-198.

Handschuh, R.F., and Litvin, F.L., 1991, "A Method for Determining Spiral-Bevel Gear Tooth Geometry for Finite Element Analysis," NASA Technical Paper 3096/AVSCOM Technical Report 91-C-020.

Hourlier, F., d'Hondt, H., Truchon, M., and Pineau, A., 1985, "Fatigue Crack Path Behavior Under Polymodal Fatigue," Multiaxial Fatigue, ASTM STP 853, K.J. Miller and M.W. Brown, Eds., American Society for Testing and Materials, Philadelphia, pp. 228-248.

Irving, P.E., and Buller, R.G., 1999, "Prediction of Fatigue Life Under Helicopter Loading Spectra for Safe Life and Damage Tolerance Design," Fatigue and Fracture Mechanics: Twenty Ninth Volume, ASTM STP 1332, T.L. Panontin and S.D. Sheppard, Eds., American Society for Testing and Materials, West Conshohocken, PA, pp. 727742.

Irwin, G.R., 1957, "Analysis of Stresses and Strains Near the End of a Crack Traversing a Plate," Journal of Applied Mechanics, Volume 24, pp. 361-364.

Jeong, D.Y., Tang, Y.H., and Orringer, O., 1997, "Damage Tolerance Analysis of Detail Fractures in Rail," Theoretical and Applied Mechanics, Volume 28, pp. 109-115.

Johnson, K.L., 1985, Contact Mechanics, Cambridge University Press, Great Britain.

Kurihara, M., Katoh, A., and Kawahara, M., 1986, "Analysis on Fatigue Crack Growth Rates Under a Wide Range of Stress Ratios," Journal of Pressure Vessel Technology, Volume 108, pp. 209-213.

Laird, C., 1967, "Mechanisms and Theories of Fatigue," Fatigue and Microstructure," pp. 149-203.

Lewicki, D.G., Handschuh, R.F., Henry, Z.S., and Litvin, F.L., 1993, "Low-Noise, High-Strength, Spiral-Bevel Gears for Helicopter Transmissions," NASA TM-106080. 
Lewicki 1995

Lewicki et al. $1997 \mathrm{a}$

Lewicki et al. $1997 \mathrm{~b}$

Lewicki et al. 1998

Litvin et al. 1991

Litvin 1994

Litvin et al. 1996

McClung et al. 1989a

McClung et al. $1989 \mathrm{~b}$

McEvily et al. 1996

McFadden 1985
Lewicki, D.G., 1995, Crack Propagation Studies to Determine Benign or Catastrophic Failure Modes for Aerospace Thin-Rim Gears, Ph.D. Dissertation, Case Western Reserve University.

Lewicki, D.G., and Ballarini, R., 1997, "Effect of Rim Thickness on Gear Crack Propagation Path," Journal of Mechanical Design, Volume 119, pp. 88-95.

Lewicki, D.G., and Ballarini, R., 1997, "Rim Thickness Effects on Gear Crack Propagation Life," International Journal of Fracture, Volume 87, pp. 59-86.

Lewicki, D.G., Sane, A.D., Drago, R.J., and Wawrzynek, P.A., 1998, "Three-Dimensional Gear Crack Propagation Studies," NASA/TM1988-208827/ARL-TR-1833.

Litvin, F.L., and Zhang, Y., 1991, "Local Synthesis and Tooth Contact Analysis of Face-Milled Spiral Bevel Gears," NASA Contractor Report 4342/AVSCOM Technical Report 90-C-028.

Litvin, F.L., 1994, Gear Geometry and Applied Theory, PTR Prentice Hall, New Jersey.

Litvin, F.L., Wang, A., and Handschuh, R.F., 1996, "Computerized Design and Analysis of Face-Milled, Uniform Tooth Height Spiral Bevel Gear Drives," NASA Technical Memorandum 107348/ARLTR-1251.

McClung, R.C., and Sehitoglu, H., 1989, "On the Finite Element Analysis of Fatigue Crack Closure - 1. Basic Modeling Issues," Engineering Fracture Mechanics, Volume 33, pp. 237-252.

McClung, R.C., and Sehitoglu, H., 1989, "On the Finite Element Analysis of Fatigue Crack Closure - 2. Numerical Results," Engineering Fracture Mechanics, Volume 33, pp. 253-272.

McEvily, A.J., Pohl, K., and Mayr, P., 1996, "Comparison of the Fractographic Features of a Carburized Steel Fractured Under Monotonic or Cyclic Loading," Materials Characterization, Volume 36, pp. 153-157.

McFadden, P.D., 1985, "Analysis of the Vibration of the Input Bevel Pinion in RAN Wessex Helicopter Main Rotor Gearbox WAK 143 Prior to Failure," Aeronautical Research Report, Number AR-004049. 
Miller et al. 1999

Murakami 1987

Newman 1976

Newman 1981

Newman 1984

Nuismer 1975

Olzak et al. 1993

Owen et al. 1983

Panasyuk et al. 1995

Paris et al. 1961
Miller, M., Rufin, A.D., Westre, W.N., and Samavedam, G., 1999, "High-Speed Civil Transport Hybrid Laminate Sandwich Fuselage Panel Test," Fatigue and Fracture Mechanics: Twenty Ninth Volume, ASTM STP 1332, T.L. Panontin and S.D. Sheppard, Eds., American Society for Testing and Materials, West Conshohocken, PA, pp. 713726.

Murakami, Y., (Eds.), 1987, Stress Intensity Factors Handbook, Pergamon Press.

Newman, J.C., Jr., 1976, "A Finite-Element Analysis of Fatigue Crack Closure," Mechanics of Crack Growth, ASTM STP 590, American Society for Testing and Materials, pp. 281-301.

Newman, J.C., Jr., 1981, "A Crack-Closure Model for Predicting Fatigue Crack Growth Under Aircraft Spectrum Loading," Methods and Models for Predicting Fatigue Crack Growth under Random Loading, ASTM STP 748, J.B. Chang and C.M. Hudson, Eds., American Society for Testing and Materials, pp. 53-84.

Newman, J.C., Jr., 1984, "A Crack Opening Stress Equation for Fatigue Crack Growth," International Journal of Fracture, Volume 24, pp. R131-R135.

Nuismer, R.J., 1975, “An Energy Release Rate Criterion for Mixed Mode Fracture," International Journal of Fracture, Volume 11, pp. 245-250.

Olzak, M., Stupnicki, R., and Wójcik, R., 1993, "Numerical Analysis of 3D Cracks Propagating in the Rail-Wheel Contact Zone," Rail Quality and Maintenance for Modern Railway Operations, Kluwer Academic Publishers, pp. 385-395.

Owen, D.R.J., and Fawkes, A.J., 1983, Engineering Fracture Mechanics: Numerical Methods and Applications, Pineridge Press Limited.

Panasyuk, V.V., Datsyshyn O.P., and Marchenko, H.P., 1995, "The Crack Propagation Theory Under Rolling Contact," Engineering Fracture Mechanics, Volume 52, pp. 179-191.

Paris, P.C., Gomes, M.P., and Anderson, W.E., 1961, "A Rational Analytical Theory of Fatigue," The Trend in Engineering, Volume 13, pp. 9-14. 
Paris et al. 1963

Parks 1974

Pehan et al. 1997

Pepi 1996

Proprietary Source 1998

Qian et al. 1996

Rice 1968

Roth et al. 1992

Rudd 1984

Savage et al. 1989

Savage et al. 1992
Paris, P.C., and Erdogan, F., 1963, "A Critical Analysis of Crack Propagation Laws," Journal of Basic Engineering, Volume 85, pp. 528-534.

Parks, D.M., 1974, "A Stiffness Derivative Finite Element Technique for Determination of Crack Tip Stress Intensity Factors," International Journal of Fracture, Volume 10, pp. 487-502.

Pehan, S., Hellen, T., Flasker, J., and Glodez, S., 1997, "Numerical Methods for Determining Stress Intensity Factors vs. Crack Depth in Gear Tooth Roots," International Journal of Fatigue, Volume 19, pp. 677-685.

Pepi, M., 1996, "Metallurgical Examination of a Failed Spiral Bevel Gear," Technology Showcase: Integrated Monitoring, Diagnostics, and Failure Prevention. Proceedings of a Joint Conference, Mobile, Alabama, April 22-26.

Qian, J., and Fatemi, A., 1996, "Fatigue Crack Growth Under MixedMode I and II Loading," Fatigue and Fracture of Engineering Materials and Structures, Volume 19, pp. 1277-1284.

Rice, J.R., 1968, "A Path Independent Integral and the Approximate Analysis of Strain Concentration by Notches and Cracks," Journal of Applied Mechanics, Volume 35, pp. 379-386.

Roth, M., and Sander, K., 1992, "Fatigue Failure of a Carburized Steel Gear From a Helicopter," Handbook of Case Histories in Failure Analysis, Volume 1, ASM International, pp. 228-230.

Rudd, J.L., 1984, "Air Force Damage Tolerance Design Philosophy," Damage Tolerance of Metallic Structures: Analysis Methods and Applications, ASTM STP 842, J.B. Chang and J.L. Rudd, Eds., American Society for Testing and Materials, pp. 134-141.

Savage, M., Altidis, P.C., Lewicki, D.G., Coy, J.J., and Litvin, F.L., 1989, "Tooth Contact Shift in Loaded Spiral Bevel Gears," NASA Technical Memorandum 101438/AVSCOM Technical Report 87-C36.

Savage, M., Prasanna, M.G., and Coe, H.H., 1992, "Maximum Life Spiral Bevel Reduction Design," NASA Technical Memorandum 105790/AVSCOM Technical Report 92-C-004. 
Schijve 1988

Schijve 1996

Sfakiotakis et al. 1997

Sih 1974

Singh et al. 1998

Swift 1984

Westergaard 1939

Williams 1957
Schijve, J., 1988, "Fatigue Crack Closure: Observations and Technical Significance," Mechanics of Fatigue Crack Closure, ASTM STP 982, J.C. Newman, Jr. and W. Elber, Eds., American Society for Testing and Materials, pp. 5-34.

Schijve, J., 1996, "Predictions on Fatigue Life and Crack Growth as an Engineering Problem. A State of the Art Survey," Fatigue '96: Proceedings of the Sixth International Fatigue Congress Volume II, Editors G. Luterjering and H. Nowak, Elsevier Science Inc., pp. 11491164

Sfakiotakis, V.G., Katsareas, D.E., and Anifantis, N.K., 1997, "Boundary Element Analysis of Gear Teeth Fracture," Engineering Analysis with Boundary Elements, Volume 20, pp. 169-175.

Sih, G.C., 1974, "Strain-Energy-Density Factor Applied to Mixed Mode Crack Problems," International Journal of Fracture, Volume 10, pp. 305-321.

Singh, R., Carter, B.J., Wawrzynek, P.A., and Ingraffea, A.R., 1998, "Universal Crack Closure Integral for SIF Estimation," Engineering Fracture Mechanics, Volume 60, pp. 133-146.

Swift, T., 1984, "Fracture Analysis of Stiffened Structure," Damage Tolerance of Metallic Structures: Analysis Methods and Application, ASTM STP 842, J.B. Chang and J.L. Rudd, Eds., American Society for Testing and Materials, pp. 69-107.

Timoshenko et al. 1970 Timoshenko, S.P., and Goodier, J.N., 1970, Theory of Elasticity, Third Edition, McGraw-Hill, Inc.

Townsend et al. 1991 Townsend, D.P., and Bamberger, E.N., 1991, "Surface Fatigue Life of M50NiL and AISI 9310 Gears and Rolling-Contact Bars," Journal of Propulsion and Power, Volume 7, pp. 642-649.

Westergaard, H.M., 1939, "Bearing Pressures and Cracks," Journal of Applied Mechanics, Volume 6, pp. 49-53.

Williams, M.L., 1957, "On the Stress Distribution at the Base of a Stationary Crack," Journal of Applied Mechanics, Volume 24, pp. 109-114. 


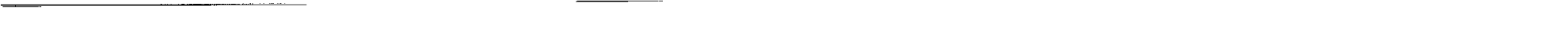




\begin{tabular}{|l|c|c|}
\hline 1. AGENCY USE ONLY (Leave blank) & $\begin{array}{c}\text { 2. REPORT DATE } \\
\text { May } 2000\end{array}$ & $\begin{array}{r}\text { 3. REPORT TYPE AND DATES COVERED } \\
\text { Final Contractor Report }\end{array}$
\end{tabular}

\section{TITLE AND SUBTITLE}

Simulating Fatigue Crack Growth in Spiral Bevel Gears

6. AUTHOR(S)

Lisa E. Spievak, Paul A. Wawrzynek, and Anthony R. Ingraffea

7. PERFORMING ORGANIZATION NAME(S) AND ADDRESS(ES)

Cornell University

Cornell Fracture Group

641 Frank Rhodes Hall

Ithaca, New York 14853

9. SPONSORING/MONITORING AGENCY NAME(S) AND ADDRESS(ES)

U.S. Amy Research Laboratory

Cleveland, Ohio 4135-3191

and

NASA Glenn Research Center

Cleveland, Ohio 44135-3191

11. SUPPLEMENTARY NOTES

Project Manager, David G. Lewicki, Structures and Acoustics Division, NASA Glenn Research Center, organization code 5950, (216) 433-3970.

\section{2a. DISTRIBUTION/AVAILABILITY STATEMENT}

12b. DISTRIBUTION CODE

NAG.3-1993
Unclassified - Unlimited

Subject Category: 37

This publication is available from the NASA Center for AeroSpace Information, (301) 62 I-0390.
WU-581-30-13-00

1L162211A47A

8. PERFORMING ORGANIZATION

REPORT NUMBER

E-12287

10. SPONSORINGMONITORING AGENCY REPORT NUMBER

NASA CR-2000-210062

ARL-CR-45I

\section{ABSTRACT (Maximum 200 words)}

The majority of helicopter transmission systems utilize spiral bevel gears to conver the horizontal power from the engine into vertical power for the rotor. Due to the cyclical loading on a gear's tooth, fatigue crack propagation can occur. In rotorcraft applications, a crack's trajectory determines whether the gear failure will be benign or catastrophic for the aircraft. As a result, the capability to predict crack growth in gears is significant. A spiral bevel gear's complex shape requires a three dimensional model of the geometry and cracks. The boundary element method in conjunction with linear elastic fracture mechanics theories is used to predict arbitrarily shaped three dimensional fatigue crack trajectories in a spiral bevel pinion under moving load conditions. The predictions are validated by comparison to experimental results. The sensitivity of the predictions to variations in loading conditions and crack growth rate model parameters is explored. Critical areas that must be understood in greater detail prior to predicting more accurate crack trajectories and crack growth rates in three dimensions are identified.

\section{SUBJECT TERMS}

Gears: Spiral-bevel gears; Crack propagation; Fracture mechanics; Boundary element method

\begin{tabular}{|c|c|}
\hline $\begin{array}{c}\text { 17. SECURITY CLASSIFICATION } \\
\text { OF REPORT }\end{array}$ & $\begin{array}{c}\text { 18. SECURITY CLASSIFICATION } \\
\text { OF THIS PAGE } \\
\text { Unclassified }\end{array}$ \\
Unclassified
\end{tabular}

NS̄N 7540-01-280-5500

19. SECURITY CLASSIFICATION
OF ABSTRACT
Unclassified

Standard Form 298 (Rev. 2-89)

Prescribed by ANSI Std. Z39-18 